Propositions

1. Microalgal cultures appear to be simple to pump, illuminate, subject to mass exchange and harvest but those processes are in reality difficult to do.

2. Microalgal cultivation and the associated science has been fluctuating between being fashionable and obsolete several times in the preceding 75 years and there are publication opportunities in both phases.

3. When justifying a statement with a reference, the author ensures that the statement in the reference is equivalent but not necessarily correct.

4. If you examine a generally accepted fact by following the tree of citations all the way down to the original paper, it is not uncommon discover that the author was incorrectly cited.

5. Uncontroversial statements may be correct but are boring.

6. The advice you give your children is often received under protest and one day they pass it on to their children.

Propositions belonging to the thesis, entitled

Development of industrial scale microalgae production

Niels-Henrik Norsker

Wageningen, 19 January 2021 

Development of industrial scale microalgae production 


\section{Thesis committee}

\section{Promotors}

Prof. Dr R. H. Wijffels

Professor of Bioprocess Engineering

Wageningen University \& Research

Prof. Dr M.J. Barbosa

Personal chair, Bioprocess Engineering

Wageningen University \& Research

\section{Other members}

Prof. Dr AJ van der Goot, Wageningen University \& Research

Prof. Dr C Vilchez, University of Huelva, Spain

Dr DMM Kleinegris, NORCE Norwegian Research Centre, Norway

Prof. Dr J Legrand, Université de Nantes, France

This research was conducted under the auspices of the Graduate School VLAG

(Advanced studies in Food Technology, Agrobiotechnology and Health Sciences). 


\title{
Development of industrial scale microalgae production
}

\author{
Niels-Henrik Norsker
}

Thesis

submitted in fulfilment of the requirements for the degree of doctor at Wageningen University

by the authority of the Rector Magnificus,

Prof. Dr A.P.J. Mol,

in the presence of the

Thesis Committee appointed by the Academic Board

to be defended in public

on Tuesday 19 January 2021

at 1.30 p.m. in the Aula 
Niels-Henrik Norsker

Development of industrial scale microalgae production 180 pages.

PhD thesis, Wageningen University, Wageningen, the Netherlands (2021)

With references, with summary in English

ISBN: 978-94-6395-662-8

DOI: https://doi.org/10.18174/537678 


\section{Chapters}

1. Introduction and thesis outline

2. Microalgal production - A close look at the economics

3. On energy balance and production costs in tubular and flat panel photobioreactors 55

4. Productivity of Nannochloropsis oceanica in an industrial closely spaced flat panel

photobioreactor

5. Developing microalgal oil production for an outdoor photobioreactor

6. Neochloris oleoabundans oil production in an outdoor tubular photobioreactor

at pilot scale

7. General discussion

8. Completed training activities

9. Publication list

10. Bibliography

11. Author curriculum vitae 
1. Introduction and thesis outline 


\section{Microalgal production development: early developments}

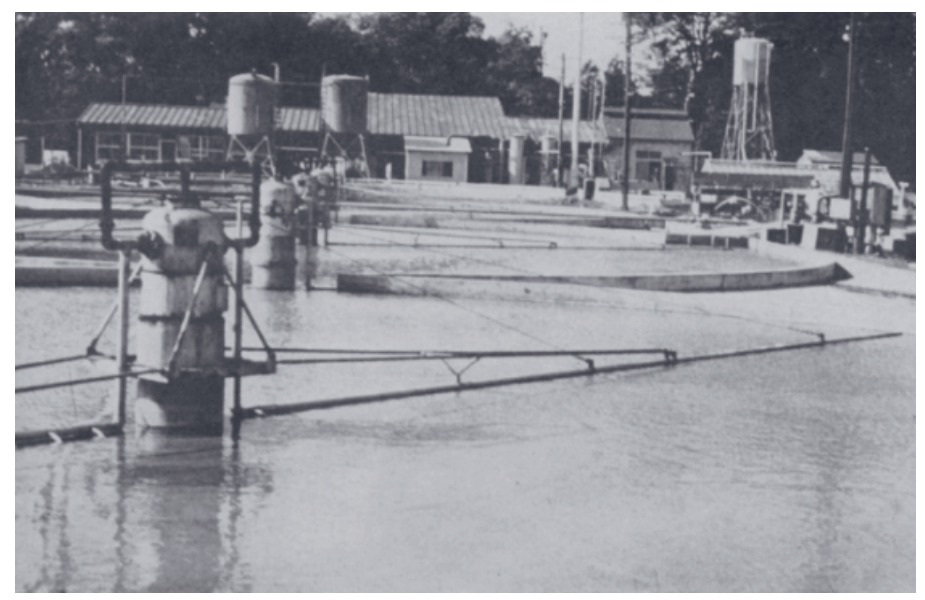

Fig. 1-1 Centre-pivot algae ponds at the Japanese Microalgae Research Institute, at Kunitachi, Japan. The pivot drives a boom that mixes the culture. (Krauss 1962)

The first attempts to exploit microalgae industrially, were during the second world war in Germany (Pulz et al. 1998). From 1942, the US Carnegie Foundation had funded research in the influence of growth conditions on the composition and growth of microalgae and the notion of microalgae as a potential major human foodsource was proposed in an annual report and this presumably was the kick-off for the first wave of microalgal production development. It is a bit of a visional experience to read the first few lines of the account: "The liaison between the physical sciences and technology has become so intimate that discoveries and developments in these sciences find applications in industry with remarkable readiness. Such is not the case in the biological sciences, or certainly to a much lesser degree" (Spoehr et al. 1947). To-day, after 7 decades of applied research and technological development, one must conclude that it is quite true. The reason that Spoehr gave - that the biological sciences are a lot more complicated that the physical, still seems solid. Ramping up a new biological technology from research to market break through often takes many decades. Industrial mariculture, for example is normally considered to have taken off in with the interest generated by the International Fishing Exhibition in London in 1883 and by fear of declining fish stocks and government funded marine fish hatcheries were established in several countries. The Swedish government arranged the 
first international mariculture conference in Stockholm in 1899 and in Christiania (later Oslo) in 1901 and that resulted in establishment of modern scientific mariculture research with the hatchery and research station at Flødevigen, Norway. International collaboration and exchange of methods and fish fry with several countries followed, including the US with the Woods Hole hatchery that had been established with government funds in 1855 (Nash 2011). Two world wars meant a halt to commercial fish farming developments but in 1970 , the first commercial salmon farm was created with the construction of a simple and robust sea cage and in 1971, 886 tons of salmon could be exported (Isabella 2020). In 2019, 1.1 million tons of salmon was exported (Norwegian_Seafood_Council 2020). It is noteworthy that Norway as a newborn independent nation managed to turn mariculture into a scientific discipline and establish a clear focus on Salmon. Norway now is an industrial giant in the field.

Industrial microalgal development was also initiated on the assumption of a looming global food supply crisis. In 1947, the first systematic investigation of large-scale Chlorella production began with a TNO project at the Laboratory for Plant Physiological Research at Wageningen University in the Netherlands in collaboration with the Carnegie Research Institution in the US. TNO is an applied research institution, founded in 1932 to enable business and government to apply knowledge (TNO 2020) so the immediate focus on photobioreactor technology in outdoor pilot scale is probably thanks to that. Very rapidly, the framework of microalgal photosynthetic production research was drawn up. In 19501951, data on photosynthetic efficiency of Chlorella in 1 m², 300 L outdoor concrete tanks were compared with similar, artificially illuminated in-door tanks. In daylight, but at rather low daily irradiation (5.1 - 7.3 $\mathrm{MJ} \mathrm{m}^{-2}$ day $^{-1}$ ), outdoor mass cultures had a photosynthetic efficiency of $2.7-2.6 \%$ (PAR) whereas in-doors cultures had an efficiency of $4.7 \%$ at $11.1 \mathrm{MJ}$ day $^{-1}$. The fact that indoors reactors had higher efficiency at higher irradiation is important as it soon was clear that the challenge lay in making the outdoor cultures convert daylight with high efficiency at high irradiation. Also, by reducing daylight irradiation to $22 \%$, the efficiency (PAR) was increased to $6.3 \%$.

In large series of small scale lab cultures, most efficiencies ranged between $12-21 \%$ (PAR) with maximum 23.5\% (PAR) (Wassink et al. 1953). 
At the time, it was not immediately clear whether the discrepancy was due to temporarily high photosynthetical efficiency in the laboratory cultures while the efficiency in normally growing cultures would drop or to suboptimal growth conditions in the experimental outdoor photobioreactors. In all circumstances, the hopes were that the operation of outdoor photobioreactors could be optimized to produce high photosynthetic efficiency also in full daylight. The work on productivity enhancement and photosynthetic efficiency was discontinued in Wageningen in the mid-1950'ies but taken up again in the late 1990'ies and Wageningen has since year 2000 published about 50 microalgal papers per year, in Europe exceeded by Paris that since 2007 reached a publication rate of 100 papers per year (Rumin et al. 2020).

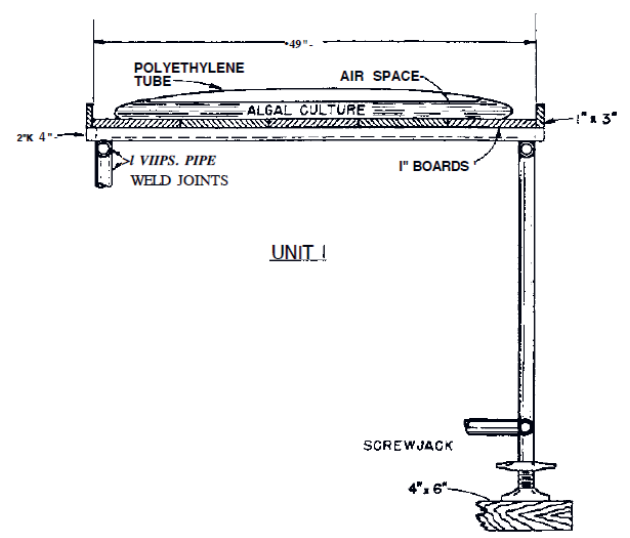

Fig. 1-2

A cross-section of the tubes from the Arthur D. Little pilot plant. The culture depth could be set by varying the filling degree of the plant. Depths of 2" to 4" were tested. Gas exchange was established through $\mathrm{CO}_{2}$-enriched, atmospheric air, injected in the head space, just after the circulation pump. Gas escape also from the tube head space (Burlew 1953)

\section{Microalgal production plants}

The first approach to an industrial microalgal production plant, was a closed, tubular photobioreactor, established by the Carnegie Research Institution and built on the rooftop of the administrative head quarter of the engineering company, Arthur D. Little in Cambridge, Massachusetts. In (Burlew 1953), there is a quite detailed description of the operational experience with the plant. This is quite unusual and valuable. Normally, very few details emerge from the operation of pilot plants, managed by private or

private-public enterprises for the reason that successful designs and procedures are guarded as proprietary knowledge while negative experiences and flat-out failures only are divulged when they later may serve to discourage potential competitors. For professionals who have been constructing and operating microalgal pilot plants, the similarity of the 
approaches and problems is striking. And publishing the accounts of the experiences could advance the technology development.

The design was a lay-flat tubular photobioreactor plant, made from partially filled, large diameter polyethylene tubes (Fig. 1-2), laid horizontally in a U-shape. This resulted in enclosed, shallow channels in which the culture was recycled by a centrifugal pump. The reactors were started at low biomass density $\left(<50 \mathrm{mg} \mathrm{dw} \mathrm{L}^{-1}\right)$ from Chlorella cultures, produced in vertical outdoor glass columns. The reactor was operated semicontinuously from July through October 1951 with an average productivity of $9 \mathrm{~g} \mathrm{dw} \mathrm{m}^{-2}$ day $^{-1}$ (based on projected tube area). No measurements of irradiation were done but daily variability in productivity was observed to appear related to weather conditions.

Soon after, pilot plants followed in Israel, Czechoslovakia, Japan and Germany (Burlew 1953, Masojídek et al. 2010).

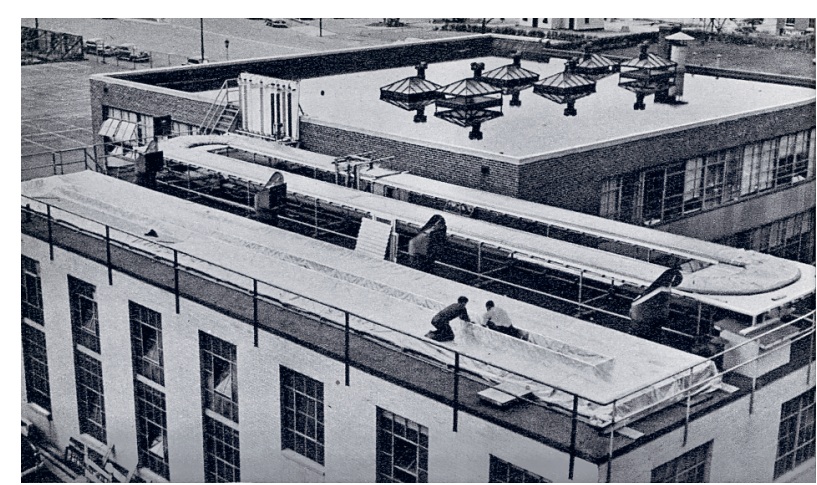

Fig. 1-3 Pilot plant under construction on the rooftop of the Arthur D. Little building. Glass columns for inoculum production seen at the adjacent building (Burlew 1953)

\section{Industrial scale production}

was first established in Japan, in 1961 (Iwamoto 2004) in connection to a state research institute ("The Chlorella Institute"). Iwamoto described the initial developments at the institute: "They struggled with many problems and

greatly contributed to the development of practical technology. Circular shallow ponds with central, rotating, tangential liquid distributor had been developed and from 1959, mixotrophy had been discovered and introduced, using acetate and that marked the threshold for successful, commercial application (administration of $\mathrm{CO}_{2}$ was costly at the time, as opposed to use of acetate). 
The Chlorella Institute had been established in the outskirts of Tokyo in 1957 and had supplied large amounts of Chlorella biomass to several nutritional research institutes in Japan. Initiating the Chlorella industry was a businessman who had become convinced about the potentials of Chlorella as a health food (Iwamoto 2004). Focus on health food and close collaboration between private enterprise and state research probably was the reason Japan succeeded in establish Chlorella production as an industry in Japan as opposed to the "dead end" for the Carnegie Research Institution project in the US. In Taiwan, shallow circular pond with pivot scraper/stirrer (Fig. 1-1) were instrumental in a rapidly developing Chlorella industry. The pivot stirrer drives a radial bar over the bottom of the tank, which serves to prevent biomass from settling. Malaysia and Indonesia soon followed suite.

The "green revolution" meant that the algal production for staple food lost interest.

In the 1970ies, 2 new applications of microalgae were examined: wastewater purification and production of shellfish with algae. A combination of the two applications seems tempting and was tested, but hygienic regulations prevents a direct coupling. Algal ponds and raceways were developed for wastewater purification in the US and widely applied (Oswald 2003). In the 1980'ies, the emerging industry, marine aquaculture needed microalgal feed for shellfish larvae and specialized photobioreactor development started several places in the US and Europe.

A new generation of photobioreactors appeared, the raceway: a ring-shaped channel with a paddlewheel to circulate the culture. Compared to the circular pond, it has the advantage of enhanced gas exchange on the paddle wheel but its advantage over the circular pond mostly related to scalability; the largest circular ponds are $50 \mathrm{~m}$ in diameter (Lee 2001) i.e. with an area of $2000 \mathrm{~m}^{2}$ whereas the largest raceways are $10,000 \mathrm{~m}^{2}$ (Borowitzka 2005) as the length of the pivot stirrer limits the size of the circular pond. The limit to the size of the raceway is constituted by the distance that the culture flows from the front side of the paddlewheel till it arrives backside of the paddle wheel. It is a question whether extending the size of the reactor beyond the 1 ha level at all is desirable - balancing the size of reactor with the risk of microalgal contamination is important; susceptibility to other fast growing 
algae is dealt with by intermittently starting the reactor with clean starter cultures and that is more difficult the larger, the reactor is.

The use of algae then took a temporary slow-down in the late 1980 'ies when inert diets became dominating in aquaculture but now aquaculture remains an important field of application of algae for tank water conditioning, feed and pigmentation.

Markets for dry microalgal biomass (mostly Spirulina and Chlorella) and carotenoids (ßcarotene and astaxanthin) for human health food and aquaculture feeds developed in the 1990 'ies. Using dry diets in aquaculture processes is not as simple as it may sound because dispersion and buoyancy and maintenance of the dietary quality of the powdered dry algae is critical. But it was a breakthrough to be able to separate microalgal production and use of the product. Important factor in establishing the market for microalgal aquaculture feeds was the improving understanding of the dietary and gnotobiological ${ }^{1}$ requirements of marine fish larvae. Aquaculture diets remains to-day an important market for high-value microalgal products.

\section{Current microalgal products.}

Currently, the global market of autotrophic micro algae is about 30,000 tons of biomass, distributed on only 7 major species (Table 1-1). These are the species that are available on the market; in addition, microalgae are used as live diets in aquatic hatcheries, for example for bivalve rearing, but these algae are mostly produced on-site. Over the past 10 years, the market has approximately tripled but sadly, it remains small, considering the large public interest in microalgae.

The species in Table 1-1 are all high-value products for human food supplements or nutraceuticals or special aquafeeds although the Japanese company by the name Euglena company intends to develop its Euglena-product for biofuel.

Two of the species, Spirulina and Dunaliella are produced in open ponds under conditions that are rather exclusive to these species, namely high alkalinity (Spirulina) and high salinity (Dunaliella). Haematococcus and Euglena are produced in photobioreactors or hybrid

\footnotetext{
${ }^{1}$ Gnotobiology denotes a controlled microbiological environment of a given organism.
} 
production technology (photobioreactors, followed by a short open-pond phase.

Nannochloropsis is predominantly produced in photobioreactors but there are some examples of open pond or hybrid technology. AFA (Aphanizomenon flos-aquae) is harvested from a freshwater lake in Oregon. Chlorella is produced with a variety of technologies, either autotrophically, mixotrophically or heterotrophically. But generally, it can be said that in order to produce algae in open ponds, conditions that are exclusive to other algae must be established in the pond.

Most new food applications of microalgae are based on Spirulina and Chlorella as these algae do not require novel food approval due to the long history of consumption of those species for food. For a broadening of the species base, expensive and lengthy novel food approval is required and until robust and cost-effective production methods in general have been developed, it is unlikely that companies are willing to accept the risks and costs of developing new microalgal products.

Table 1-1 Current global production of photosynthetic microalgae (Olaizola et al. 2019)

\begin{tabular}{|l|l|}
\hline Species & Tons per year \\
\hline Spirulina & 18,000 \\
\hline Chlorella & 9500 \\
\hline Dunaliella & 1700 \\
\hline AFA & 500 \\
\hline Haematococcus & 300 \\
\hline Nannochloropsis & 150 \\
\hline Euglena & 50 \\
\hline & \\
\hline Total & 30,206 \\
\hline
\end{tabular}

\section{Emerging areas of application}

To-day, mankind is challenged by many interconnected sustainability issues. Microalgal production may contribute to building a green economy in direct and in-direct ways. 


\section{Atmospheric $\mathrm{CO}_{2}$ reduction and industrial sustainability}

Atmospheric $\mathrm{CO}_{2}$ concentrations are steadily climbing (Fig. 1-4). During the 1990 'ies, annual atmospheric carbon emission has on the average been $6.3 \pm 0.4 \mathrm{Pg}^{2}$, with $3.2 \pm 0.1$ accumulating in the atmosphere and $1.7 \pm 0.5 \mathrm{Pg}$ being absorbed by the oceans and $1.4 \pm$ 0.5 on land (Prentice et al. 2001).

$\mathrm{CO}_{2}$ absorbed in the oceans are causing acidification with potential detrimental effects for various zooplankton and shellfish. Sustainability would require ocean $\mathrm{CO}_{2}$ accumulation to be halted.

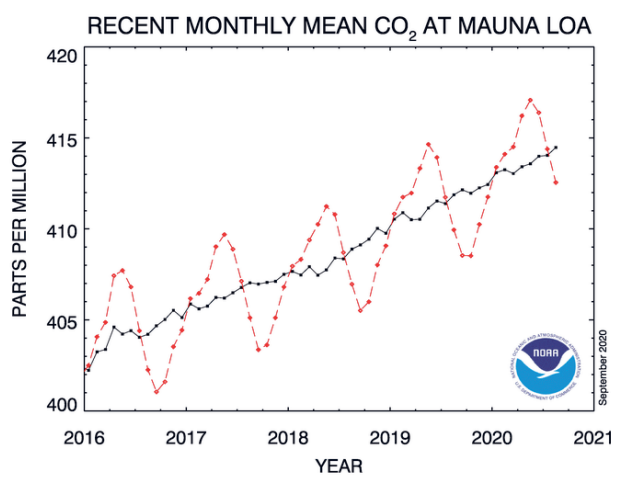

Fig. 1-4 Atmospheric $\mathrm{CO}_{2}$ level variation, measured by the US National Oceanic and Atmospheric Administration at Mauna Loa, Hawaii. The steady upwards trend is noticeable but also the annual cyclic variation that is caused by the assimilation of the terrestrial plant cover at the northern hemisphere (NOAA 2020).

Microalgal production as a direct $\mathrm{CO}_{2}$ abatement tool was a driver behind many microalgal research projects in the first decade of the new millennium and particularly, the ability to grow with a high load of flue gasses was investigated. This coupling lost interest, probably because of the strong scale incompatibility between the industrial emitters such as power plants and cement factories and $\mathrm{CO}_{2}$ uptake capacity in microalgal production plants, a Danish cement factory in 2017 for example emitted an annual average of $2.17 \times 10^{6}$ tons of $\mathrm{CO}_{2}$ during the years 2016-2018 (Aalborg-Portland 2018); with an expected annual biomass productivity of 40 tons ha ${ }^{-1} \mathrm{y}^{-1}$, the microalgal cultivation area, required to absorb the emissions from the factory, would be around 32,000 ha or corresponding to $1.2 \%$ of the total Danish arable land area. Allocating areas in the size of just a few thousand hectares in near vicinity to power stations or major industries would be difficult. But probably also contributing to the drop of

\footnotetext{
${ }^{2} \mathrm{Pg}=$ petagram $\left(10^{15} \mathrm{~g}=10^{9}\right.$ ton $)$
} 
enthusiasm about large scale microalgal $\mathrm{CO}_{2}$ abatement, were a number of LCA analyses of microalgal biofuel production. Standardizing the system boundaries, $\mathrm{CO}_{2}$ emissions from microalgal biodiesel use were rather similar to rapeseed biodiesel and worse for some tubular reactor scenarios. (Review of 7 LCA studies (Slade et al. 2013)). Furthermore, if microalgal production were to have any influence on the $\mathrm{CO}_{2}$ emission from a coal-fired power plant, it would have had to be very large and placed in close vicinity to the power plant.

The annual atmospheric zig-zag $\mathrm{CO}_{2}$ trend (Fig. 1-4) is an indication of the strong effect of terrestrial photosynthesis on atmospheric $\mathrm{CO}_{2}$ levels: the annual $\mathrm{CO}_{2}$ decrease in driven by assimilation in the vegetation while decay of terrestrial plants contributes to the annual increase. This influence comes mainly from the northern hemisphere that has the largest landmasses. Stopping and reversing deforestation could result in building up carbon storage in natural forests for a period long enough for atmospheric $\mathrm{CO}_{2}$ to come down to safe levels. Developing microalgal production of feedstocks that currently are the reason for deforestation, such as protein replacements for soy and alternatives to palm oil, could help reversing the deforestation. At the moment, heterotrophic algae production is closer to attain a scale which is relevant in that connection

But with heterotrophic cultivation of microalgae it is important to keep in mind that production of sugar that is used for Schizochytrium has a quite large $\mathrm{CO}_{2}$-footprint: $400 \mathrm{~kg}$ $\mathrm{CO}_{2}$ per ton sugar if it is produced on existing crop land and much more if based on land, reclaimed from forests (Brenton et al. 2010).

From the microalgal production side, $\mathrm{CO}_{2}$ provision has always been a heavy cost factor, so the connecting these two activities is attractive. Mandatory carbon capture applied to large $\mathrm{CO}_{2}$ emitters could be an opportunity to reduce the cost of microalgal production. With sufficient sustainable electrical power, we can afford spending energy on microalgal cultivation - it is not primarily the energy expenditure, that is the problem but the source of energy. Currently, there is an almost desperate struggle to find methods for storage of sustainable electrical energy: the probably most promising future technology to store and use wind energy for cars, the electrofuels or power-to-liquid, only have a $13 \%$ energy conservation efficiency as opposed to $73 \%$ with direct battery storage (Buffet 2017) and the cost is currently 3-7 higher than fossil fuels (Gates 2020). And yet these pre-conditions 
do not discourage investments in the technology. Recalling the words of Spoehr, we have to keep in mind that biological techniques are complicated and may require a longer haul.

\section{Sustainable food production for an increasing population}

It is interesting to read a presentation from the 50 years' anniversary of Wageningen University in 1968 by professor E.C. Wassink, from the Laboratory for Plant Physiological Research, Wageningen University. Motivated by concern for the disappearance of wildlife and degradation of natural habitats, Wassink argued that an agricultural area of $4 \mathrm{Mkm}^{2}$ with integrated agriculture (including livestock), horticulture and silviculture and with primary production taking $25 \%$ of the area, would suffice to support the entire future human population estimate ( 5 billion). This area would constitute only $3 \%$ of the global land surface. Large scale fish culture and microalgal cultures should be researched (Wassink 1970).

In 2011, food production took up $35 \%$ of the total land surface and livestock constituted $80 \%$ of the agricultural area. If the entire human population should adopt US diet, the agricultural land use would lead to an increase $178 \%$, even though the US meat now constitutes $30 \%$ of the diet, down from 38\% in 1970 (Alexander et al. 2016). Now 50 years have passed since Wassink gave his speech; the problems are more acute than ever, and the concerns are still the same. Main research domain in European microalgal/phytoplankton papers is environment with 8962 out of 26137 papers and food is second with 4275 papers. (Rumin et al. 2020).

Plant-based food is gaining increased acceptance in parts of the industrialized world. Germany, for example that traditionally consumed rather little vegetables and fruits, has now reached a tipping point where (42\%) would say they were deliberately limiting meat consumption while less people said they were comfortable with eating meat (Oltermann 2020). This has a bearing on microalgal production. Since 3 to 4 years, many microalgal $R \& D$ projects have started operating within meat replacements and soy protein replacement. Whereas microalgal biofuel production depends on triacylglycerol formation that requires growth limitation, protein formation takes place at fast biomass growth rates and that would unleash the high microalgal productivity potential. Microalgal protein in a multiple product value chain already now appears economically viable (Slegers et al. 2020). 


\section{Water management}

Water management is a global issue of ever-increasing importance. In arid regions, producing food at low water consumption is a subsistence condition. In Israel, for example algal cultivation is considered a desert technology. Heat tolerant species (42 degrees) may be cultivated in open ponds during summertime at a water loss of $20 \mathrm{~L} \mathrm{~m}^{-2}$ day $^{-1}$, even in hot desert areas where positive convection (heating) occurred during night (Pruvost et al. 2019). With a modest heat-exchanger in the ground under the ponds, both heat tolerant and temperate species could be cultivated year-round. Cooling with saltwater, either from aquifers or from the sea is another option. In a 1 ha flat panel plant near Livorno, Italy, annual power expenditure for pumping sea water for cooling, was $52 \mathrm{GJ} \mathrm{J}^{-1} \mathrm{ha}^{-1}$. (Tredici et al. 2015) or $14,400 \mathrm{kWhr}$. This is very little, compared with the energy output of the produced biomass, $799 \mathrm{GJ} \mathrm{y}^{-1} \mathrm{ha}^{-1}$ in the $36 \mathrm{t}$ Tetraselmis net energy ratio however was still negative, mostly because of a large power consumption for bubbling the cultures $(317.1 \mathrm{GJ}$ $\mathrm{y}^{-1} \mathrm{ha}^{-1}$ ). The Proviron reactor (Fig. 1-5), (Norsker et al. 2019) offers an elegant solution to temperature control in temperate climates where heating is dominated by radiation - the panels are enclosed in a water filled bag which is large enough to absorb heat from a high irradiation (>30 $\mathrm{MJ} \mathrm{m}^{-2}$ ) summer day and dissipate it during night. These examples hold promise - there are technical and economical solutions for maintaining adequate temperature in both open and enclosed microalgal cultures.

\section{Land utilization}

Microalgae may be produced in closed reactors on industrially impacted land that is unsuitable for agricultural crops and such activity is therefore not in competition with conventional agricultural food production. An example is the flat panel photobioreactor plant, that from 2011 to 2017 was integrated in a solid waste disposal plant, Hooghe Maey in Antwerp. The plant was developed by the Belgian chemical company, Proviron. The waste disposal plant contributed hot water and electrical power and $\mathrm{CO}_{2}$ from biogas combustion to the algal plant (Fig. 1-5), (Norsker et al. 2019). Open pond cultivation of algae, on the other hand is sensitive to airborne and water pollution from industrial activities. 


\section{Wastewater treatment}

Microalgal cultivation may turn wastewater treatment energy positive, yielding energy upon methanization of the produced biomass. Currently, wastewater treatment in the US takes $3 \%$ of the electrical power produced (Shoener et al. 2014). Algal ponds and lagoons are quite common wastewater treatment technology in the US, particularly in rural areas because of their large footprint, the focus is on COD reduction and removal of nitrogen and phosphorous and metals. Indeed, retention of nitrogen and phosphorous was suggested by W.J. Oswald in use of combined bacterial-microalgal wastewater treatment ponds, he pioneered in the US in the mid 1950'ies. In the US, there are to-day more than 7000 wastewater treatment facilities in operation that use facultative algae ponds (EPA 2002). Because of their high depth (1-1.5 m), facultative ponds have both anaerobic and aerobic breakdown of organic matter, with microalgae providing oxygen for bacterial respiration of COD and assimilate $\mathrm{N}$ and $\mathrm{P}$. High rate oxidation ponds are raceway ponds, similar to the ponds used for Spirulina production etc.

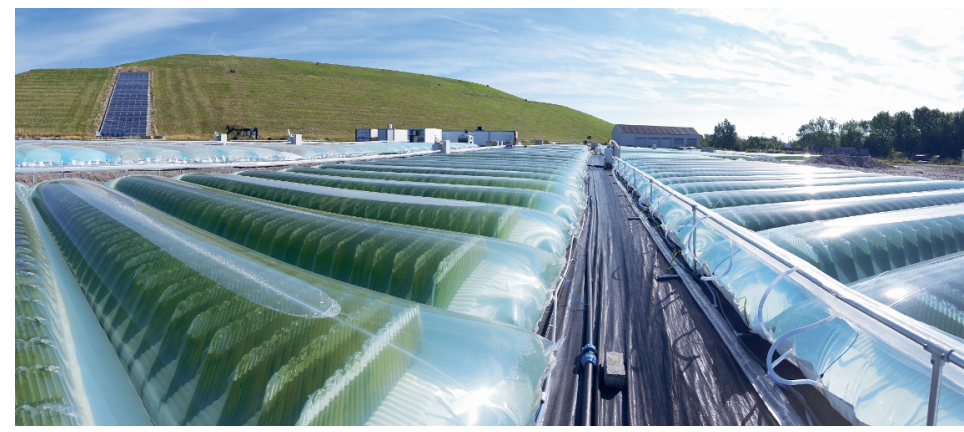

Fig. 1-5 The Proviron flat plate photobioreactor plant, installed on the municipal solid waste treatment plant, Hooghe Maey in Antwerp in 2015. The hills in the back of the picture are membrane covered, solid organic waste heaps, covered with a membrane. They produce biogas that is combusted in gas motor driven, electrical power generators. Photograph: courtesy of Proviron.

Also algal rotating-disc biofilm plants (Christenson et al. 2011, Johnson et al. 2018), algal turf scrubbers (Mulbry et al. 2008, Wollmann et al. 2019) and other algal biofilm processes are being developed as well as photobioreactors (Michels et al. 2014, Krishnamoorthy et al. 2019). Compared with anaerobic technology plants, phototrophic wastewater treatment processes have a considerable potential to become net energy producers with 
methanization of the produced biomass. Turf scrubbers are readily energy positive while some other microalgal processes require energy reduction of mixing- and aeration operations (Shoener et al. 2014).

\section{Biofuel production}

During 2008 to 2014, crude oil prices were around \$100 per barrel (Fig. 1-6) and gave rise to the peak oil notion, which was a belief that the oil ressources were dwindling and the world was fast running out of oil. It raised the expectations that microalgae might generate a replacement of fossil oil for automotive fuel - mainly biodiesel. Combining unconstrained microalgal growth rates with high density-large volume cultures and stationary-phase oil contents led to unrealistically high oil production projections - one company for example claimed productivities that would have amounted to an energy conservation of $11.5 \mathrm{MJ} \mathrm{m}^{-2}$ day $^{-1}$, or an efficiency of $50 \%$ the insolation on a sunny summer day (Tredici et al. 2010). This is one order of magnitude larger than possible. Almost all microalgal biofuel projects have ceased operating or changed to other more economically viable strategies. ExxonMobile - Synthetic Genomics have maintained their microalgal biofuel development strategy, based on genetically modified Nannochloropsis gaditana (Lane 2018) and Japanese Euglena progresses in a jet fuel development plan (Okutsu 2018), while currently producing Euglena for other high value purposes. Jet fuel might be an area where microalgal biofuels may become viable and attractive compared with other alternatives. 
In all circumstances, biofuels is one of the significant focus areas for European microalgae papers with 1014 papers published out of 26132 (Rumin et al. 2020).

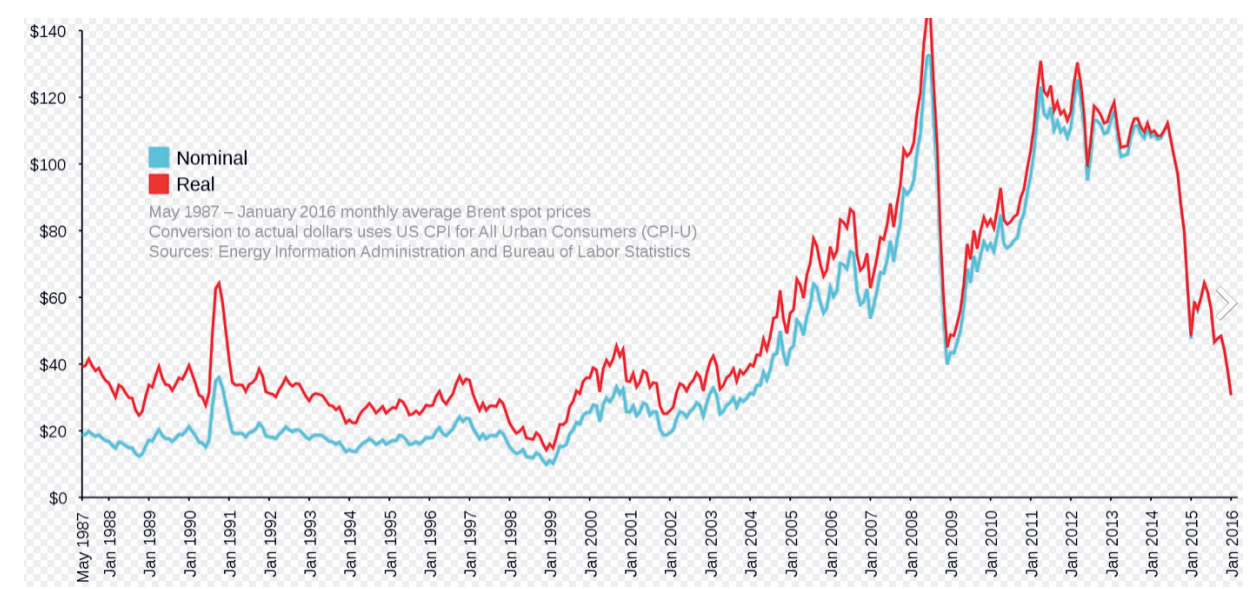

Fig. 1-6 Brent crude prices 1987 - 2015. - (Wikipedia 2020). With exception of the period 2009-2010 with the impact of the financial crisis, crude oil prices had been close to $\$ 100$ / barrel from 2008 to 2015.

\section{Outline of the work and reseach questions adressed:}

This thesis is titled "Development of industrial scale microalgae production" and addresses aspects from the technical - economical analysis of photobioreactor performance in chapter 2 and 3, modeling and validation of biomass production in a flat panel reactor in chapter 4 and development and validation of oil production in a tubular photobioreactor in chapters 5 and 6 . The achievements of the work is discussed in chapter 7 which also tries to answer the question why industrial microalgal production progresses so slowly.

Research questions adressed

The research aims to answer following questions:

1. Based on current process- and technical photobioreactor knowledge (2012) and available experimental photobioreactor data:

a. What are realistic industrial scale-biomass production costs with ponds, tubular- and flat panel photobioreactors, respectively? 
b. Which are the essential categories in the biomass production cost and what are the potentials for cost reduction in these categories?

2. What are the sources to auxiliary energy consumption in the examined photobioreactors and how and to what level could the energy comsumption be reduced?

3. How does biomass productivity of a flat panel photobioreactor theoretically depend on local irradiation conditions and temperature at a north European location?

4. Can photobioreactor technology be used to produce oil from microalgal biomass?

\section{Chapter 2: In the fulfillment of research question 1.}

The chapter describes a projection of the biomass production costs with the 3 different production plant types for 2 different locations: the Netherlands and southern Spain. Monthly irradiation averages from meteorological database tables were used as input and biomass production were estimated from average photosynthetic efficiency data from the available pilot plant studies of the 3 different types of production plant. The results were presented as unit costs per $\mathrm{kg}$. biomass produced and for simplicity grouped in a small number of categories. A sensitivity analysis was carried out, estimating the effect of future reductions of various cost items. The results are published as (Norsker et al. 2011)

The projections were met with large interest at many conferences and meetings and has with currently 700 citations (Google scholar) been useful for the microalgal community.

\section{Chapter 3: In the fulfillment of research question 2.}

From around 2009, papers on microalgal biofuels started to be published, following the efforts to find alternatives to mineral oil, that the "peak oil notion" created.

3 main types of microalgal production plants were being adressed: open raceway ponds, tubular photobioreactors and flat-panel photobireactors. As energy production was the objective, the energy budget of the microalgal production was essential. At the time, papers were being published, citing very high mixing energy costs for tubular photobioreactors - up to a level of $6 \mathrm{~kW} \mathrm{~m}^{-3}$. Aeration in flat panel reactors, on the other hand appeared to be heavily under underestimated. So, there was basis for looking the 
mixing costs for the two reactor types with different equipment and different scale. Chapter 2 of the thesis is standard hydraulic calculation of a) the power supply for liquid circulation in a tubular mannifold reactor with variable liquid velocity and amount of biofilm in the tubes and b) the aeration power supply in flat panel reactors. The results are published as (Norsker et al. 2012) .

\section{Chapter 4: In the fulfillment of research question 3, with emphasis on a flat-panel photobioractor.}

In 2008, a collaborative project with the Belgian Chemical Industry Proviron and 2 other industry partners, was established.

The research objective was to support the development of a closely spaced, flat panel photobioreactor produced from plastic film and hence in principle disposable.

Disposing, though was expected to take place no more frequently than necessary, and early financial projections were based on a lifetime of 12 months.

The closely spaced flat panel reactor was inspired by design work of Otto Pulz, (Pulz et al. 1995) who had obtained very high volumetric productivities $\left(1.3 \mathrm{~g} \mathrm{~L}^{-1} \mathrm{day}^{-1}\right)$ with a closely spaced, flat panel reactor, although the exact conditions under which this volumetric productivity had been obtained, have not been published. Light dilution, at the time, was seen as the method to obtain high photosynthetic efficiency under natural daylight conditions. In all circumstances, the project plan included that a computer model should be developed to project to obtain a clear idea as to which productivity could be expected. The model work could also be used to help the design - particularly panel height and distance between them and orientation of the reactorproductivity under different irradiation and design conditions in order. The choice of species had not been set in advance. Experiments with cultivation of different algal species in a single Proviron panel in a water filled aquaria were performed to verify general panel performance, mass transfer and productivity were carried out (Fig. 1-7). After trials with Phaeodactylum, a switch was made to Nannochloropsis. The selected strain, Nannochloropsis sp. CCAP 211/78 was later named as a Nannochloropsis oceanica. A $400 \mathrm{ml}$ thermostated experimental flat panel reactor with adjustable, red (630 $\mathrm{nm}$ ) LED illumination reactor was used with dilute culture to obtain a 
set of temperature-irradiance dependent growth rate data for N. oceanica. Generation of these data took more than 1.5 years to make. A light-temperature model was fitted to the data, resulting in a growthrate model, depending on the 2 variables, light intensity and temperature. A ray tracing model was developed to calculate the light intensity (from daylight) on the panel surface in any of 20 vertical segments. This was depending on the

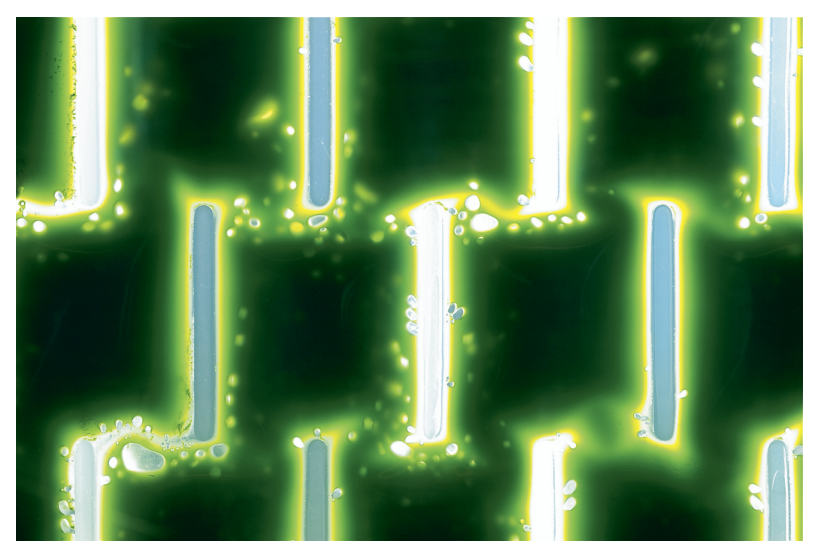

Fig. 1-7 Proviron panel with a high density culture of Nannochloropsis. The panel shown was an early version with "ice-cube bag" welding pattern. distribution of direct and diffuse light. Light profile inside the panels was calculated, using the integrated Lambert-Beer formula, with absorption coefficient measured for low light adapted $N$. oceanica in an integrating sphere. Cloudiness calculation was made by comparing actual horizontal irradiation with conventionally calculated irradiation and the cloudiness could then be used to estimate the proportion of direct-diffuse light.

Growth rates at given light intensity and temperature inside the panels, could now be calculated for the Proviron reactor panels and converted to instantaneous productivity for the entire reactor, based on actual light measurement-temperature model from the 400 $\mathrm{ml}$. reactor. So, with measured horizontal irradiation and temperature - which, due to the design of the Proviron reactor was surprisingly homogeneous, information about orientation of reactor and geographical position - degrees with 2 decimals necessary the instantaneous reactor productivity over the day could modelled. The model productivity was validated with $\mathrm{CO}_{2}$ absorption-based productivity data for a number of typical days of the year in Antwerp. The results are published in (Norsker et al. 2019) 


\section{Chapters 5 and 6: in the fulfillment of reseach question 4.}

In 2010, a practical study of the reliability and cost of the most promising technology for commodities from microalgae was started; in the first instance represented only by biomass. We opted for the flat horizontal tubular reactor with disposable soft-sleeve tubes. The basic design was developed by us and the plant built by the environmental technology producer, Paques. $6 \mathrm{~cm}$ diameter polyethylene tubing with a wall thickness of $175 \mu \mathrm{m}$ was used. The plant had 4 manifold lanes, 2 to each side of the main outgoing pipe; each with 4 outgoing tubes in the middle of the lane and 4 returning tubes at the side. The objective with this arrangement was to ensure an equal velocity in all the tubes, which succeded. The selected species, Neochloris (Ettlia) oleoabundans was propagated axenically in a 10 liter glass CSTR.

Evaporative cooling requirements of the plant with almost $2 \mathrm{~km}$ of plastic tubing was first examined empirically with black-colored water, foam- and bubble formation in the tubing appeared to constitute also a shading problem in addition to flow resistance and was solved with antifoam substance. Dissolved oxygen turned out to be difficult to drive out of the medium with bubbling with atmospheric air, but a falling film degassing principle was discovered to be more efficient. The installed tube connections to the manifold studs turned out to be unsafe and the tubing frequently popped off and new fixture had to be devised. Establishing the minimum biomass densities at inoculation was done in a photobioreactor with standard fluorescence measurement. After 4 months, $35 \mathrm{~kg} \mathrm{dw}$ Neochloris and a local strain of Scenedesmus had been produced in the plant as a $20 \% \mathrm{dw}$ slurry. An extensive report with the findings was made.

Early 2011, the project was extended with the objectives to produce a number of batches with optimized oil content from of a minimum of two strains, selected from promising oil producing candidates.

10 microalgal candidates were selected and tested for oil formation in shake flasks. Oil formation rate and yield were measured. Neochloris oleoabundans and Chlorococcum littorale were selected. Introducing nutrient limitation in the $6 \mathrm{~m}^{3}$ tubular photobioreactor in order to enhance the oil content is a challenge. In 2010, preliminary data on Neochloris 
indicated that there was a simple mathematical relation between oil content and the cell nitrogen ratio - the amount of nitrogen, assimilated per biomass unit. In 2011, the relation was established for Neochloris and Chlorococcum and it was tested in practice in the photobioreactor with all the potential interferences - photorespiration, photoinhibition, heat- and light gradients and unpredictable cloudiness effects. 4 batches were produced. Daily data on dry weight, residual nitrogen and phosporous and fatty acid content and distribution were collected. 


\title{
2. Microalgal production - A close look at the economics
}

Published as: Norsker, N.-H., M. J. Barbosa, M. H. Vermuë and R. H. Wijffels (2011). Microalgal production - A close look at the economics. Biotechnology Advances 29: 24-27.

\begin{abstract}
Worldwide, microalgal biofuel production is being investigated. It is strongly debated which type of production technology is the most adequate. Microalgal biomass production costs were calculated for 3 different micro algal production systems operating at commercial scale today: open ponds, horizontal tubular photobioreactors and flat panel photobioreactors. For the 3 systems, resulting biomass production costs including dewatering, were 4.95, 4.15 and $5.96 €$ per $\mathrm{kg}$, respectively. The important cost factors are irradiation conditions, mixing, photosynthetic efficiency of systems, medium- and carbon dioxide costs. Optimizing production with respect to these factors, a price of $€ 0.68$ per $\mathrm{kg}$ resulted. At this cost level microalgae become a promising feedstock for biodiesel and bulk chemicals.
\end{abstract}




\section{Background}

Microalgal biomass has been suggested as an energy source for a number of compelling reasons, including high area yields compared with other crops, high oil content in some strains, low water consumption and the possibility of production on arid lands and several oil companies, including Exxon, BP, Chevron, Shell and Neste Oil (BP, 2009; Mascarelli, 2009) are investing in research in microalgae for energy purposes. In a recent study, microalgal fuel production was concluded to be relatively close to being economi- cally feasible, given expected developments in market conditions and production technology (Stephens et al., 2010). But the scientific community in the field is divided with respect to the question which of the microalgal production technology is the most promising for future developments and scale up? It has been claimed that photobioreactors are unsuited for biomass production at a cost compatible with biofuel production (Waltz, 2009) whereas open systems suffer from low biomass productivity and high costs of biomass harvesting because of low biomass densities, large land use, losses of carbon dioxide and poor contaminant control possibilities (Posten, 2009). Microalgae can conserve a maximum of 9-10\% of solar the solar energy (photosynthetic efficiency) but microalgal outdoor production systems so-far rarely exceed 6\% (Carvalho et al., 2006). However, new methods for genetic modification and metabolic flux modelling of microalgae are being developed and are believed to result in higher photosynthetic efficiency (Schenk et al., 2008; Beer et al., 2009; Wijffels et al., 2010). In continuously illuminated systems, microalgae can already now be cultivated at high efficiency at light intensities similar to or higher than direct sunlight (Qiang et al., 1998; Cuaresma et al., 2009), but growth in the dynamic light environment in outdoor systems is still far from being well understood. In the last decade, there has been a private sector development of new, low cost photobioreactor systems that apply the light dilution principle which has been shown give high photosynthetic efficiency (Pulz and Scheibenbogen, 1998) and the development of biorefinery processes will contribute to the value chain (Wijffels et al., 2010). What is missing, however, is a conceptual model for assessment of the effect of enhancing photosynthetic efficiency on one side and increased operating 
costs on the other. The present work is attempting to fill that gap by assessing the performance of present day photobioreactors and analyzing the effect of optimizing key process parameters on biomass production costs.

\section{Algal reactors}

The cost of microalgal biomass production under Dutch climatic conditions was analyzed for the 3 main types of photobioreactor systems that are commercially applied today: open raceway ponds, tubular photobioreactors and flat panel photobioreactors. A short description of the main operational characteristics of the 3 reactors is given here but a full description of the process layout and energy cost analysis can be found in the Supplementary material: Design basis.pdf.

The raceway ponds are shallow, ring-channel systems, in which the depth for hydraulic reasons cannot be less than $0.2 \mathrm{~m}$. This results in a low biomass density ( $0.3 \mathrm{~g}$ DW per liter). To provide mixing, the culture is circulated with a paddle wheel at a velocity of $0.25 \mathrm{~m} / \mathrm{s}$. The process requires significantly less energy for mixing than the two other reactor designs but due to low biomass density, high costs are incurred for harvesting. This type of reactor is extensively used in industrial microalgal production, for example to produce Spirulina and Dunaliella of which globally $5000 \mathrm{t}$ and $1200 \mathrm{t}$, respectively, is produced per year (Spolaore et al., 2006).

In the tubular reactor, the algal culture is circulated in transparent tubes by a centrifugal pump and intermittently passes through a degasser - an air-sparged vessel where the accumulated oxygen is blown off. High oxygen concentrations reduce the algal productivity and optimizing the degassing is an important feature of the design process. A horizontal layer of soft, disposable polyethylene tubing with a one-year lifetime was considered, but there are many other possible configurations such as stacked layers of glass or acrylic tubing. Turbulent flow 0.5 $\mathrm{m} / \mathrm{s}$ ) is required to mix the cells between illuminated zones at the tube periphery and dark zones around the center. With the present configuration, a biomass density of $1.7 \mathrm{~g}$ DW per liter is obtained. Tubular reactors are used industrially for 
example for producing the valuable pigment, astaxanthin with alga Haematococcus and also for Chlorella and Nannochloropsis production.

The flat panel reactor is basically a flat, transparent vessel in which mixing is carried out directly in the reactor with air sparging. The normal aeration level for flat panel photobioreactors is 1 liter of air per liter reactor volume per minute (Sierra et al., 2008). The design examined here is the closely spaced, vertical flat panel reactor, in which light dilution is obtained by applying larger specific surface and self-shading of the panels. In this way, it is possible to achieve a higher photosynthetic efficiency, albeit at higher mixing and installation costs. The panels are assumed made of polyethylene film with a one-year lifetime. The polyethylene film is supported by a steel mesh casing. The reactor configuration results in a biomass concentration of 2.1 g DW per liter. Such a system has been demonstrated efficient for example for production of algal strains that accumulate lipid under nutrient limitation (Rodolfi et al., 2009).

\section{Light and light absorption}

Calculation of productivity was based on an assessment of attainable photosynthetic efficiency for the 3 reactor systems, obtained from literature data covering various sites, algal species and time of year. The results can be found in the Supplementary material, Photosynthetic efficiency.pdf. From the presented data, PE is independent of irradiation level but depending on photobioreactor type.

Characteristic PE values for the three photobioreactor types were selected as indicated in Figs. 1-3 in the Supplementary material, Photosynthetic efficiency.pdf and the values are given here in Table 2-1.

Table 2-1 Base case: microalgal biomass production in various photobioreactor types in the Netherlands.

\begin{tabular}{llll}
\hline & Open pond & Tubular bioreactor & Flat panel bioreactor \\
\hline PE (solar) & $1.5 \%$ & $3 \%$ & $5 \%$ \\
$\begin{array}{c}\text { Productivity (ton DW) } \\
\text { per ha) }\end{array}$ & 21 & 41 & 64 \\
\hline
\end{tabular}


In Fig. 2-1, the daily horizontal irradiation for Eindhoven, the Netherlands is compared with the irradiation for Bonaire, the Dutch Antilles, which served as an example of a "near-ideal" algal cultivation site. Algal productivity was calculated for the 3 systems on a monthly basis from $\mathrm{PE}$, algal biomass combustion enthalpy and irradiation and shown in Table 2-1.

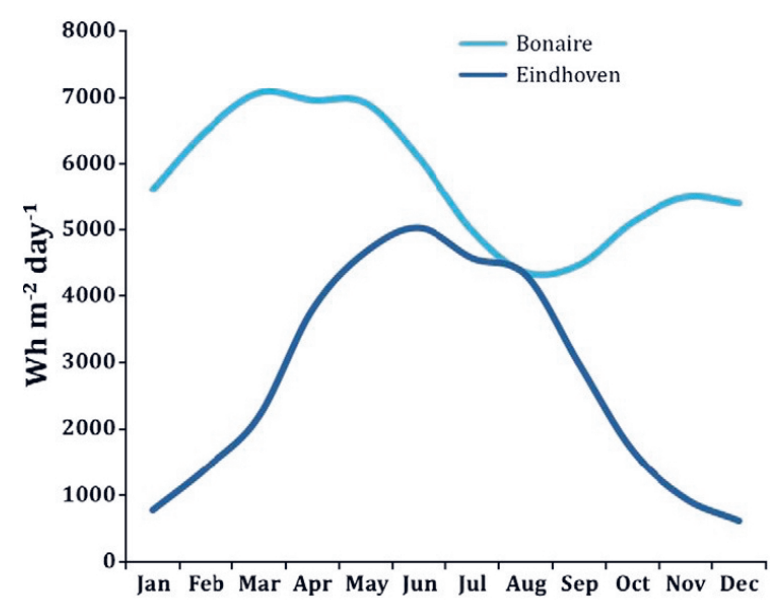

Fig. 2-1 Daily irradiation (monthly average) for Eindhoven, the Netherlands and Bonaire, the Dutch Antilles. Eindhoven data were extracted from average radiation data from 1996 to 2000(Satel-light, 2008) and for Bonaire, the 2008 record was used (MDNA, 2008).

\section{Production cost}

The distribution of biomass cost on individual items for the 3 reactor systems are presented in Table 2-2. System specific critical cost contributions are underlined these are the costs that are relatively high for the given system. Details can be downloaded from the Supplementary material:

Design basis.pdf. The calculation was carried out at plant scales 1 and 100 ha. These figures represent a base case in terms of productivity and unit operation costs.

The sum of the unit production costs is the total biomass production cost (produced as a wet paste). In the base case, the tubular photobioreactor is the most economic for production of biomass under Dutch conditions with a production cost of $€ 4.15$ per $\mathrm{kg}$ DW.

Mixing and mass transfer (oxygen removal) costs are the sum of depreciation and energy consumption of paddle wheel, circulation- and aeration pumps. The 3 plants have very different operating economy with respect to mixing. For the raceway pond, mixing costs $€ 0.08$ per kg DW. For the tubular reactor, $€ 1.27$ and for the flat panel reactor, $€ 3.10$ per kg DW. 
In the tubular reactors, mixing is created by turbulence in the circulating algal suspension. But turbulence is expensive to produce in terms of energy input and depreciation of the pumps. If high velocities are applied "to be on the safe side", the power requirement is also considerable as is evident from Fig. 2-2 in the Supplementary material, Design basis.pdf. In the base case, a velocity of $0.5 \mathrm{~m} / \mathrm{s}$ was applied. Optimizing fluid velocity in the pipes is obviously important but can only be achieved by studying the direct effects of mixing on algal growth and unfortunately, little work has been carried out in this field.

Table 2-2 Unit biomass production cost (in cts, eurocents) from various capital and operating cost elements for raceway ponds, tubular photobioreactors and flat panel photobioreactors.

\begin{tabular}{|c|c|c|c|c|c|c|}
\hline \multirow[t]{2}{*}{ (Base case) } & \multicolumn{2}{|c|}{$\begin{array}{l}\text { Raceway ponds } \\
\text { cts } \mathrm{kg}^{-1} \mathrm{DW}\end{array}$} & $\begin{array}{l}\text { Tubulars } \\
\text { cts kg-1 }\end{array}$ & DW & \multicolumn{2}{|c|}{$\begin{array}{l}\text { Flat panels } \\
\text { cts } \mathrm{kg}^{-1} \mathrm{DW}\end{array}$} \\
\hline & 1 ha & 100 ha & 1 ha & 100 ha & 1 ha & 100 ha \\
\hline \multicolumn{7}{|l|}{ Major equipment+ power } \\
\hline PVC liner & 49.33 & 40.45 & & & & \\
\hline Centrifuge & $\underline{118.66}$ & $\underline{44.45}$ & 43.26 & 9.54 & 38.61 & 7.23 \\
\hline Power & $\underline{17.02}$ & $\underline{19.12}$ & 3.65 & 3.96 & 2.54 & 2.99 \\
\hline Medium preparation & 81.31 & 44.66 & 29.29 & 9.29 & 19.31 & 7.01 \\
\hline Power & 3.80 & 4.20 & 0.84 & 0.81 & 0.64 & 0.61 \\
\hline Harvest buffer tank & 25.11 & 18.84 & 6.28 & 3.89 & 4.09 & 2.94 \\
\hline Culture circulation pump & & & 73.74 & 73.33 & & \\
\hline Power & & & $\underline{47.06}$ & $\underline{47.06}$ & & \\
\hline Steel framework & & & & & 11.73 & 11.73 \\
\hline Blower/paddle wheel & 4.52 & 4.53 & 6.91 & 0.99 & 73.55 & 69.30 \\
\hline Power & 3.17 & 3.18 & 5.83 & 5.79 & $\underline{240.67}$ & $\underline{240.67}$ \\
\hline \multicolumn{7}{|l|}{ Other capital } \\
\hline Installation costs & 41.84 & 22.94 & 47.84 & 29.11 & 44.19 & 29.46 \\
\hline Instrumentation costs & 27.89 & 15.29 & 15.95 & 9.70 & 14.73 & 9.82 \\
\hline Piping & 83.68 & 45.88 & 47.84 & 29.11 & 44.19 & 29.46 \\
\hline Buildings & 83.68 & 45.88 & 47.84 & 29.11 & 44.19 & 29.46 \\
\hline \multicolumn{7}{|l|}{ Variable costs (ex. power) } \\
\hline Polyethylene tubing/sheet & & & 12.76 & 12.76 & 9.76 & 9.76 \\
\hline Culture medium & 44.00 & 44.00 & 44.00 & 44.00 & 44.00 & 44.00 \\
\hline Carbon dioxide & 33.67 & 33.67 & 33.67 & 33.67 & 33.67 & 33.67 \\
\hline Medium filters & 44.42 & 44.42 & 18.39 & 18.39 & 13.88 & 13.88 \\
\hline Labour & 579.55 & 12.56 & 289.78 & 6.28 & 188.58 & 4.09 \\
\hline Salary overhead & 144.89 & 3.14 & 72.44 & 1.57 & 47.15 & 1.02 \\
\hline Maintenance & 42.91 & 23.53 & 49.07 & 29.86 & 45.32 & 30.22 \\
\hline General plant overheads & 342.35 & 19.85 & 93.39 & 17.09 & 128.65 & 18.87 \\
\hline Sum & 1772 & 495 & 990 & 415 & 1049 & 596 \\
\hline
\end{tabular}


Another important matter for the mixing economy in tubular reactors, is the shear stress experienced in the pump. Because of concern for shear stress damage, air-lift pumps have frequently been applied, but they are considerably less efficient than centrifugal pumps, and efficiency values as low as $2 \%$ have been recorded for tubular reactor studies (Hall et al., 2002) but unless very shear sensitive algae are produced, there is little point in applying air-lift pumps. The diatom, Chaetoceros muelleri which is widely used in aquaculture is considered relatively shear sensitive and has a shear stress tolerance slightly over $1 \mathrm{~Pa}$ (Michels et al. 2010) but is still reported to be able to tolerate the use of centrifugal pumps. We have envisaged the use of low shear centrifugal pumps. Such pumps are relatively costly and with better knowledge about shear stress tolerance in algae, pumping costs could be reduced significantly but this was not considered in the present study.

For the flat panel reactors, the cost is strongly affected by airflow and power efficiency and cost of the blower. A screw blower with a power efficiency of $45 \%$ was considered but it is possible that better economy may be obtained with other types of blowers. 


\section{Sensitivity analysis}

A sensitivity analysis of the effects of reduction of mixing costs, improvement of irradiation and photosynthetic efficiency and reduction of nutrient costs was carried out. With the tubular reactor, the effect of reducing flow velocity from 0.5 to $0.3 \mathrm{~m} / \mathrm{s}$ results in a pumping cost reduction of $€ 1.1$ per $\mathrm{kg} \mathrm{DW}$ or $26 \% .0 .3 \mathrm{~m} / \mathrm{s}$ was adopted as a minimum velocity as it will suffice for gas transfer for most combinations of algal species and climatic conditions and flow still will be within the turbulent regime. With respect to aeration in the flat panel reactor, a value of $0.3 \mathrm{~min}^{-1}$ will be enough to secure sufficient gas exchange, even under optimum growth conditions (Sierra et al.,

Table 2-3 Sensitivity analysis. Biomass cost in $€$ per $\mathrm{kg}$ with different scenarios (100 ha plant)

\begin{tabular}{|c|c|c|c|}
\hline & $\begin{array}{l}\text { Raceway } \\
\text { ponds }\end{array}$ & $\begin{array}{l}\text { Tubular } \\
\text { photobioreactors }\end{array}$ & $\begin{array}{l}\text { Flat p: nel } \\
\text { photo ioreactors }\end{array}$ \\
\hline & $(€ / \mathrm{kg} \mathrm{DW})$ & $(€ / \mathrm{kg} \mathrm{DW})$ & $(€ / \mathrm{kg} D W)$ \\
\hline 1. Base case & 4.95 & 4.16 & 5.96 \\
\hline 2. Bonaire location & 2.83 & 2.44 & 3.26 \\
\hline 3. Minimum mixing & & 3.06 & 3.08 \\
\hline $\begin{array}{l}\text { 4. } \mathrm{CO}_{2} \text { free (in addition } \\
\text { to } 3 \text { ) }\end{array}$ & 4.61 & 2.72 & 2.74 \\
\hline $\begin{array}{l}\text { 5. Medium free (in } \\
\text { addition to } 4 \text { ) }\end{array}$ & 4.17 & 2.28 & 2.30 \\
\hline $\begin{array}{l}\text { 6. PE increase } 60 \% \text { (in } \\
\text { addition to } 5 \text { ) }\end{array}$ & 2.61 & 1.43 & 1.44 \\
\hline $\begin{array}{l}\text { 7. Bonaire location (in } \\
\text { addition to } 6 \text { ) }\end{array}$ & 1.28 & 0.70 & 0.68 \\
\hline
\end{tabular}

2008) in a system dimensioned like the present flat panel reactor.

Reducing aeration to $0.3 \mathrm{~min}^{-1}$, a biomass production cost reduction of $48 \%$ is obtained.

We consider these improvements realistic in short term (about 10 years from now). The results are shown in Table 2-3. Invoking all improvements resulted in an algal production cost of about $€ 0.70$ per kg DW for the two bioreactors and $€ 1.30$ for the raceway. A price in this region readily makes the algal biomass interesting for the production of bulk products with a wide range of applications (chemical, fuels, materials, feed). 
Looking at the biomass production costs in terms of energy (Table 2-4), the best scenario results in energy cost of around $€ 25$ per GJ. This figure does not include extraction and refinery.

Table 2-4 Sensitivity analysis. Biomass cost in € per GJ, with different scenarios (100 ha plant)

\begin{tabular}{|c|c|c|c|}
\hline & $\begin{array}{l}\text { Raceway } \\
\text { ponds }\end{array}$ & $\begin{array}{l}\text { Tubular } \\
\text { photobioreactors }\end{array}$ & $\begin{array}{l}\text { Flat panel } \\
\text { photobioreactors }\end{array}$ \\
\hline & $(€ / G j)$ & $(€ / G j)$ & $(€ / G j)$ \\
\hline 1. Base case & 162.79 & 157.52 & 226.53 \\
\hline 2. Bonaire location & 107.00 & 92.12 & 123.29 \\
\hline 3. Minimum mixing & & 115.71 & 116.38 \\
\hline $\begin{array}{l}\text { 4. } \mathrm{CO}_{2} \text { free (in addition } \\
\text { to } 3 \text { ) }\end{array}$ & 174.86 & 102.86 & 103.63 \\
\hline $\begin{array}{l}\text { 5. Medium free (in } \\
\text { addition to } 4 \text { ) }\end{array}$ & 158.06 & 86.07 & 86.74 \\
\hline $\begin{array}{l}\text { 6. PE increase } 60 \% \text { (in } \\
\text { addition to } 5 \text { ) }\end{array}$ & 98.40 & 53.40 & 53.82 \\
\hline $\begin{array}{l}\text { 7. Bonaire location (in } \\
\text { addition to } 6 \text { ) }\end{array}$ & 47.95 & 25.78 & 25.00 \\
\hline
\end{tabular}

The figure is roughly similar to the cost of delivered electrical energy. Although not competitive with present day fossil fuels, it is possible to see a future role for algal biofuels, for example, as aviation fuels where the demand for compact energy sources will remain. 


\section{Supplementary material}

\section{Design basis for photobioreactors.}

\section{Power requirement for the production of compressed air:}

Power requirement for large industrial low pressure blowers were assumed applied; for the tubular reactors, a pressure of about 0.3 bar is required; hence the performance of a cyclo blower, producing $55 \mathrm{~m}^{3} \mathrm{hr}^{-1}$ per kW at 5 psig (0.34 bar) (Industrial_Assessment_Center Year?) was selected as an example to estimate the power requirements for the compressed air for the algal cultivation. At 0.15 bar which is used for the calculation of power requirements for the flat panel reactors, the performance was assumed to be 110 $\mathrm{m} 3 \mathrm{hr}^{-1} \mathrm{~kW}^{-1}$. This yield is equivalent with a power efficiency of $45 \%$. We have not been able to identify suitable blowers with higher power efficiency at the given pressure requirements, but it is possible that the use of roots blowers that may result in higher efficiency might be a more economical solution.

Both for the flat panel reactors and for the degasser with the tubular reactors, the air for sparging is assumed coarsely filtered only and not sterile filtered, contrary to the practice in some existing photobioreactor plants. Sterile filtration requires a pressure drop of several bar and would therefore ad significantly to the operating costs.

\section{Power requirements for liquid circulation in the tubular reactor.}

The specific power requirement per cubic meter tube volume (Fig. 2-2) for circulation of the culture in the tubular photobioreactor was calculated according to standard hydrodynamic principles (Coulson et al. 1999).

The tubular photobioreactor was considered constructed as a manifold system with $50 \mathrm{~m}$ tubes, with pressure drops consequently originating from friction loss from $100 \mathrm{~m}$ straight tube and from 2 manifold T-junction entries and $2 \mathrm{~T}$-junction exits. Other pressure drop contributing elements in the system, such as valves, bends and unions were considered negligible. 
The pressure drop calculation for the straight tube was done according to the Darcy-

Weisbach eqution, assuming smooth tubes and estimating the friction factor, $f=0.006$

from a Moody chart with Reynolds number of 19,000.

$$
\Delta P_{f}=8 f \frac{L}{d} \frac{\rho v^{2}}{2}
$$

Additional pressure drop in the tubular photobioreactor comes from entries and exits from the manifold tube; and can be calculated via the velocity head loss:

Velocity head, $h_{v}=\frac{v^{2}}{2 g}$

Equation 2-2

Velocity head loss due to manifold T-junctions for a manifold loop consists of velocity head loss for $2 \mathrm{~T}$-junctions $s_{\text {in }}$ and $2 \mathrm{~T}$-junctions out $_{\text {with }}$ respective velocity head loss factors 1.2 and 1.8 (Coulson et al. 1999).

$\Delta h_{v}=h_{v} 2(1.2+1.8)$

Equation 2-3

Pressure drop due to manifold elements, $\Delta_{\mathrm{p}}$, is:

$\Delta P_{v}=\Delta h_{v} \rho g$

Equation 2-4

with $\rho=$ density of liquid and g gravitational acceleration.

Total pressure drop in the photostage of the reactor (considering that there is no static height pressure drop):

$\Delta P=\Delta P_{f}+\Delta P_{v}$ Equation 2-5

The power to circulate the culture in the tubular reactor is: 
Power $=\frac{\Delta P}{\eta \rho} m$

Equation 2-6

With $\mathrm{m}$ : mass flow rate in $\mathrm{kg}$ (culture) $\sec ^{-1}$ in a given reactor section and $\eta$ : pump efficiency.

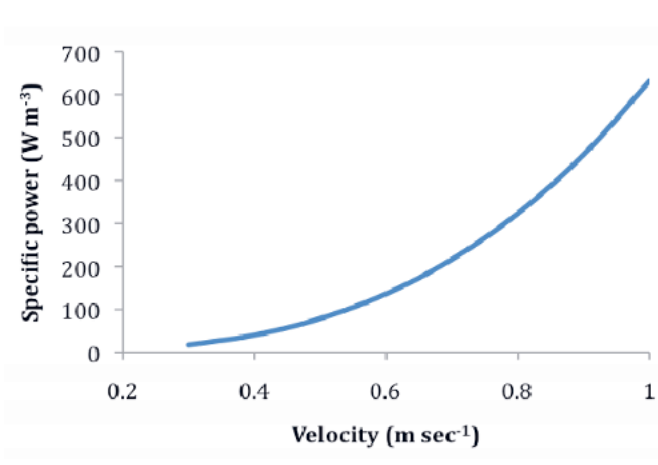

Fig. 2-2. Specific power requirements for circulating the culture in the tubes with centrifugal pump at varying velocity.

Efficiency of pump + motor $\equiv 60 \%$.
Pump efficiencies depend on pressure drop in system and pump characteristics: in the present system, the total calculated pressure drop, $\Delta P$, is $11.6 \mathrm{kPa}$ or about $1.2 \mathrm{~m}$ water column and efficiencies for centrifugal pumps of about 0.7 may be expected (Coulson et al. 1999) but efficiency values as high as 0.85 is possible with some impeller pumps.

A conservative efficiency estimate of 0.6 was applied and includes both pump and electromotor efficiency.

\section{Principle for assessment of capital items}

Capital items costs were set according to normal principles in chemical engineering(Humphreys 1991) and all three plants were treated uniformly except that installation costs for the open pond was set to $50 \%$ of the installation costs for the closed photobioreactors. 
Table 2-5. General conditions applying to all photobioreactor scenarios.

\begin{tabular}{|c|c|c|}
\hline Item & Value & Source \\
\hline Powercost & $€ 0.05 \mathrm{kWhr}^{-1}$ & \\
\hline Interest & $3 \%$ p.a. & \\
\hline Depreciation & $10 \%$ p.a. & \\
\hline Land cost & $10 € m^{-2}$ & \\
\hline $\mathrm{CO}_{2}$ cost & $€ 184$ ton $^{-1}$ & $\begin{array}{l}\text { Ben Amotz, Israel, 2007. Personal } \\
\text { communication }\end{array}$ \\
\hline Medium premix cost & $€ 0.44 \mathrm{~kg}^{-1}$ & (Grima et al. 2003) \\
\hline $\begin{array}{l}\text { Cost of polyethylene tubing for } \\
\text { tubular rectors }\end{array}$ & $€ 0.03 \mathrm{~m}^{-1}$ & Technogrow, the Netherlands. \\
\hline $\begin{array}{l}\text { Cost of polyethylene sheet for } \\
\text { flat panel reactors }\end{array}$ & & $\begin{array}{l}\text { Based on data from Technogrow, } \\
\text { the Netherlands }\end{array}$ \\
\hline Steel for construction & $€ 650$ ton $^{-1}$ & (Pimentel et al. 2005) \\
\hline Medium filters & $€ 95$ per cartridge & (Grima et al. 2003) \\
\hline Centrifuges & $\begin{array}{l}\text { Various capacities and prices, } \\
\text { see table } 1-3\end{array}$ & $\begin{array}{l}\text { Westfalia and Ben Amotz, } 2007 . \\
\text { Personal communication }\end{array}$ \\
\hline Pumps at plants & $\begin{array}{l}\text { Various capacites and prices, see } \\
\text { table } 1-3\end{array}$ & (DACE 2002) \\
\hline Medium filter unit & $€ 13,500$ & (Grima et al. 2003) \\
\hline Tanks & Various sizes and cost & Interviewed aquacuture experts \\
\hline Weighing station & $€ 40,000$ & $\begin{array}{l}\text { Interviewed biotech industry } \\
\text { expert }\end{array}$ \\
\hline Salary overheads & $25 \%$ of salaries & (Grima et al. 2003) \\
\hline Maintenance & $4 \%$ major equipment & (Grima et al. 2003) \\
\hline General plant overheads & $\begin{array}{l}55 \% \text { (salaries + salary } \\
\text { overheads) }\end{array}$ & (Grima et al. 2003) \\
\hline Blowers & $\begin{array}{l}\text { Various dimensions and prices, } \\
\text { see table 1-3 }\end{array}$ & $\begin{array}{l}\text { (DACE 2002) and various supplier } \\
\text { information }\end{array}$ \\
\hline
\end{tabular}


Table 2-6. Design basis for raceway plant.

\begin{tabular}{|c|c|c|c|}
\hline Specification & & Value & Comment \\
\hline Total land area & $\mathrm{ha}^{-1}$ & 1.25 & illuminated area $=80 \%$ \\
\hline Location & & $\begin{array}{l}\text { Eindhoven; } \\
\text { Netherlands }\end{array}$ & $\begin{array}{l}\text { Of significance for } \\
\text { irradiation }\end{array}$ \\
\hline Photosynthetic Efficiency & $\%$ & 1.5 & Solar \\
\hline Photosynthetic Efficiency & $\%$ & 3.5 & PAR \\
\hline Productivity & ton $\mathrm{ha}^{-1}$ year $^{-1}$ & 21 & Based on total land area \\
\hline Capacity, energy equivalent & GJ year-1 & 542 & \\
\hline \multicolumn{4}{|l|}{ Dimensions } \\
\hline Pond number & $\mathrm{ha}^{-1}$ & 9 & \\
\hline Volume, ponds & $\mathrm{m}^{3}$ & 200 & \\
\hline Distance between ponds & $\mathrm{m}$ & 1.1 & \\
\hline Pond length & $\mathrm{m}$ & 100 & \\
\hline Pond width & $\mathrm{m}$ & 10 & \\
\hline Pond depth & $\mathrm{m}$ & 0.2 & \\
\hline Pond area & $\mathrm{m}^{2}$ & 1000 & \\
\hline Velocity in ponds & $\mathrm{m} \mathrm{sec}^{-1}$ & 0.25 & \\
\hline Volume / ground area & $\mathrm{L} \mathrm{m}^{-2}$ & 180 & \\
\hline Total surface : volume ratio & $\mathrm{m}^{-1}$ & 5 & \\
\hline \multicolumn{4}{|l|}{ Process Design } \\
\hline Daily dilution & $\%$ & 10 & \\
\hline Volume produced per hour (12 h) & $\mathrm{m}^{3} \mathrm{ha}^{-1} \mathrm{~h}^{-1}$ & 15 & \\
\hline Volume processed per hour ( $24 \mathrm{~h})$ & $\mathrm{m}^{3} \mathrm{ha}^{-1} \mathrm{~h}^{-1}$ & 7.5 & \\
\hline Biomass concentration & $\mathrm{kg} \mathrm{m}^{-3}$ & .32 & \\
\hline Biomass production rate & $\mathrm{kg} \mathrm{m}^{-3} \mathrm{~h}^{-1}$ & 0.003 & $12 \mathrm{~h}$ day \\
\hline \multicolumn{4}{|l|}{ Major utilities } \\
\hline $\mathrm{CO}_{2}$ requirement & & 1.8 & $\begin{array}{l}\text { ton } \mathrm{CO}_{2} / \text { ton biomass } \\
\text { produced }\end{array}$ \\
\hline Net $\mathrm{CO}_{2}$ binding in plant & ton ha-1 year-1 & 38 & \\
\hline Power for cell harvest & $\mathrm{kWhr} \mathrm{m}^{-3}$ & 1.1 & \\
\hline Power requirement for paddle wheel & kW ha-1 & 5 & $24 \mathrm{~h}_{\text {day }}^{-1}$ \\
\hline Culture medium premix & ton $\mathrm{ha}^{-1}$ year $^{-1}$ & 20.7 & \\
\hline Medium filter cartridges & ha $^{-1}$ year $^{-1}$ & 132 & \\
\hline
\end{tabular}


Table 2-7. Cost of items for raceway ponds

\begin{tabular}{|c|c|c|c|c|c|c|c|}
\hline \multirow{2}{*}{$\begin{array}{l}\text { Major equipment (MEC) } \\
\text { Scale }\end{array}$} & \multirow[b]{2}{*}{ ha } & \multicolumn{2}{|c|}{$\begin{array}{l}\text { Maximum capacity } \\
\text { or value }\end{array}$} & \multicolumn{2}{|c|}{ Cost per unit $(€)$} & \multicolumn{2}{|c|}{ Number of units ha-1 } \\
\hline & & 1 & 100 & 1 & 100 & 1 & 100 \\
\hline PVC & $\mathrm{m}^{2}$ & 10,991 & $9,011,011$ & 7.15 & 7.15 & 10,991 & 9,011 \\
\hline Centrifuge Westfalia separator & $m^{3} h^{-1}$ & 22 & 60 & 183,000 & 500,000 & 1 & 0.14 \\
\hline Centrifuge Feed Pump & $m^{3} h^{-1}$ & 25 & 80 & 6,000 & 8,000 & 1 & 0.1 \\
\hline Medium Filter Unit & $m^{3} h^{-1}$ & 6 & 6 & 13,500 & 13,500 & 1 & 1.4 \\
\hline Medium Feed pump & $m^{3} h^{-1}$ & 25 & 60 & 6,000 & 8,000 & 1 & 0.14 \\
\hline Medium preparation tank & $\mathrm{m}^{3}$ & 60 & 60 & 20,000 & 20,000 & 2 & 1.5 \\
\hline Harvest broth storage tank & $\mathrm{m}^{3}$ & 60 & 60 & 20,000 & 20,000 & 2 & 1.5 \\
\hline Seawater pump station & $\mathrm{m}^{3} \mathrm{~h}^{-1}$ & 6 & 6 & 15,000 & 15,000 & 2 & 1.4 \\
\hline $\begin{array}{l}\text { Automatic Weighing Station } \\
\text { with silos }\end{array}$ & & & & 40,000 & 40,000 & 1 & 0.01 \\
\hline Paddle wheel & & & & 800 & 800 & 9 & 9 \\
\hline \multicolumn{8}{|l|}{ Operating cost items } \\
\hline Labor & staff ha-1 & 3 & 7 & 40,000 & 37,100 & 3 & 0.07 \\
\hline Payroll charges & $\%$ salary & 25 & 25 & & & & \\
\hline Maintenance & $\%$ MEC & 2 & 2 & & & & \\
\hline $\begin{array}{l}\text { General plant overheads } \\
\text { (Labor+ maintenance) }\end{array}$ & $\%$ & 55 & & & & & \\
\hline \multicolumn{8}{|l|}{ Other fixed costs } \\
\hline Installations costs & $\% \mathrm{MEC}$ & \multicolumn{2}{|l|}{15} & & & & \\
\hline Instrumentation & $\% \mathrm{MEC}$ & \multicolumn{2}{|l|}{10} & & & & \\
\hline Piping & $\%$ MEC & \multicolumn{2}{|l|}{30} & & & & \\
\hline Buildings & $\%$ MEC & \multicolumn{2}{|l|}{30} & & & & \\
\hline $\begin{array}{l}\text { Land (land cost not included as } \\
\text { capital, but interests on land are } \\
\text { included). }\end{array}$ & $€ \mathrm{~m}^{-2}$ & \multicolumn{2}{|l|}{10} & & & & \\
\hline Investment per ha (tot land) & $\mathrm{M} €$ & 0.68 & 0.37 & & & & \\
\hline
\end{tabular}


Table 2-8. Design basis for horizontal tubular photobioreactor

\begin{tabular}{|c|c|c|c|}
\hline Specification & & Value & Comment \\
\hline Total land area & $\mathrm{ha}^{-1}$ & 1.25 & illuminated area $=80 \%$ \\
\hline Location & & $\begin{array}{l}\text { Eindhoven, } \\
\text { Netherlands }\end{array}$ & Determining for irradiation \\
\hline Photosynthetic Efficiency & $\%$ & 3 & Solar based \\
\hline Photosynthetic Efficiency & $\%$ & 7.1 & PAR based \\
\hline Productivity & ton ha-1 year $^{-1}$ & 41 & Total land area \\
\hline Capacity, energy equivalent & GJ year-1 & 1085 & \\
\hline \multicolumn{4}{|l|}{ Dimensions } \\
\hline Reactor covered area & ha & 1 & Tube covered area \\
\hline Tube diameter & $\mathrm{m}$ & 0.057 & \\
\hline Velocity in tubes & $\mathrm{m} \mathrm{sec}^{-1}$ & 0.5 & \\
\hline Flow rate in tubes & $m^{3} h^{-1}$ & 4.59 & \\
\hline Volume, tube + degasser & $\mathrm{m}^{3} \mathrm{ha}^{-1}$ & 497 & \\
\hline Volume, tubes & $\mathrm{m}^{3} \mathrm{ha}^{-1}$ & 448 & \\
\hline Total surface : volume ratio & $\mathrm{m}^{-1}$ & 22.3 & \\
\hline Volume / ground area & $\mathrm{L} \mathrm{m}^{-2}$ & 44.8 & \\
\hline Volume produced per hour & $\mathrm{m}^{3} \mathrm{ha}^{-1} \mathrm{~h}^{-1}$ & 6.21 & $12 \mathrm{~h}$ production day \\
\hline \multicolumn{4}{|l|}{ Process Design } \\
\hline Daily dilution & $\%$ & 15 & \\
\hline Biomass production rate & $\mathrm{kg} \mathrm{m}^{-3} \mathrm{~h}^{-1}$ & 0.021 & $12 \mathrm{~h}$ production day \\
\hline Biomass concentration & $\mathrm{kg} \mathrm{m}^{-3}$ & 1.7 & \\
\hline Air flow rate in degasser & $\mathrm{m}^{3} \mathrm{~min}^{-1} \mathrm{~m}^{-3}$ & 0.1 & Volume air:volume culture \\
\hline $\begin{array}{l}\text { Culture volume processed per } \\
\text { hour }\end{array}$ & $m^{3} h a^{-1} h^{-1}$ & 3.11 & $24 \mathrm{~h}$ processing day \\
\hline $\mathrm{O}_{2}$ concentration at tube inlet & $\%$ air saturation & 133 & \\
\hline Culture circulation time & $\min$ & 3.3 & \\
\hline \multicolumn{4}{|l|}{ Major utilities } \\
\hline $\mathrm{CO}_{2}$ requirement & & 1.8 & $\begin{array}{l}\text { ton } \mathrm{CO}_{2} / \text { ton biomass } \\
\text { produced }\end{array}$ \\
\hline $\mathrm{CO}_{2}$ net binding in plant & ton $\mathrm{CO}_{2}$ ha $^{-1}$ year $^{-1}$ & 76 & \\
\hline Polyethylene tubing & $\mathrm{m}$ ha $^{-1}$ year $^{-1}$ & 175,438 & $@ € 0.03$, annual replacement \\
\hline Culture circulation pump power & $\mathrm{kW} \mathrm{m}^{-3} \mathrm{~min}$ & 0.3 & \\
\hline Blower power & $\mathrm{m}^{3} \mathrm{~h}^{-1} \mathrm{~kW}^{-1}$ & 55 & 0.39 bar \\
\hline Power for cell harvest & $\mathrm{kWh} \mathrm{m} \mathrm{m}^{-3}$ & $0.9-1.1$ & \\
\hline Culture medium premix & ton ha-1 year $^{-1}$ & 41 & \\
\hline Medium filters & cartridges ha-1 year ${ }^{-1}$ & 109 & \\
\hline
\end{tabular}


Table 2-9. Cost of items, tubular photobioreactor

\begin{tabular}{|c|c|c|c|c|c|c|c|}
\hline \multirow{2}{*}{$\begin{array}{l}\text { Major equipment (MEC) } \\
\text { Scale }\end{array}$} & \multirow[b]{2}{*}{ ha } & \multicolumn{2}{|c|}{ Maximum capacity } & \multicolumn{2}{|c|}{ Cost / Unit (€) } & \multicolumn{2}{|c|}{ Number of units ha-1 } \\
\hline & & 1 & 100 & 1 & 100 & 1 & 100 \\
\hline Circulation pump & $\mathrm{m}^{3} \mathrm{~h}^{-1}$ & 1000 & 1000 & 26,100 & 26,100 & 9 & 8.9 \\
\hline Centrifuge Westfalia separator & $\mathrm{m}^{3} \mathrm{~h}^{-1}$ & 11 & 60 & 133,000 & 500,000 & 1 & 0.06 \\
\hline Centrifuge Feed Pump & $\mathrm{m}^{3} \mathrm{~h}^{-1}$ & 12.5 & 80 & 4,800 & 8,000 & 1 & 0.05 \\
\hline Medium Filter Unit & $m^{3} h^{-1}$ & 5.99 & 5.99 & 13,500 & 13,500 & 1 & 0.57 \\
\hline Medium Feed pump & $\mathrm{m}^{3} \mathrm{~h}^{-1}$ & 12.5 & 80 & 4,800 & 8,000 & 1 & 0.05 \\
\hline Medium preparation tank & $\mathrm{m}^{3}$ & 60 & 60 & 20,000 & 20,000 & 1 & 0.62 \\
\hline Harvest storage tank & $\mathrm{m}^{3}$ & 60 & 60 & 20,000 & 20,000 & 1 & 0.62 \\
\hline Seawater pump station & $\mathrm{m}^{3} \mathrm{~h}^{-1}$ & 6 & 6 & 15,000 & 15,000 & 1 & 0.58 \\
\hline Weighing station & & & & 40,000 & 40,000 & 1 & 0.01 \\
\hline Air blowers & $m^{3} h^{-1}$ & 1,488 & 12,000 & 22,000 & 105,000 & 1 & 0.03 \\
\hline \multicolumn{8}{|l|}{ Operating cost items } \\
\hline Labor & staff ha-1 & 3 & 7 & 40,000 & 37,142 & 3 & 0.07 \\
\hline Payroll charges & $\%$ salary & \multicolumn{2}{|l|}{25} & & & & \\
\hline Maintenance & & 4 & $\% \mathrm{MEC}$ & & & & \\
\hline General plant overheads & $\%$ & \multicolumn{2}{|l|}{55} & & & & \\
\hline \multicolumn{8}{|l|}{ Other fixed costs } \\
\hline Installations costs & $\%$ MEC & \multicolumn{2}{|l|}{30} & & & & \\
\hline Instrumentation and control & $\%$ MEC & \multicolumn{2}{|l|}{10} & & & & \\
\hline Piping & $\% \mathrm{MEC}$ & \multicolumn{2}{|l|}{30} & & & & \\
\hline Buildings & $\%$ MEC & \multicolumn{2}{|l|}{30} & & & & \\
\hline $\begin{array}{l}\text { Land (land cost not included as } \\
\text { capital, but interests on land are } \\
\text { included). }\end{array}$ & $€ \mathrm{~m}^{-2}$ & \multicolumn{2}{|l|}{10} & & & & \\
\hline Investment per ha (total land) & $M €$ & 0.84 & 0.51 & & & & \\
\hline
\end{tabular}


Table 2-10. Design basis for flat panel photobioreactor.

\begin{tabular}{|c|c|c|c|}
\hline Specification & & Value & Comment \\
\hline Total land area & ha $^{-1}$ & 1.25 & illuminated area $=80 \%$ \\
\hline Orientation & & Facing south & \\
\hline Location & & $\begin{array}{l}\text { Eindhoven, } \\
\text { Netherlands }\end{array}$ & for irradiation data \\
\hline Photosynthetic Efficiency & $\%$ & 5 & Solar based \\
\hline Photosynthetic Efficiency & $\%$ & 11.8 & PAR based \\
\hline Productivity & ton ha-1 year-1 & 64 & Base: total land area \\
\hline Capacity, energy equivalent & GJ year-1 & 1667 & \\
\hline \multicolumn{4}{|l|}{ Dimensions } \\
\hline Reactor covered ground & ha & 1 & \\
\hline Distance between panels & $\mathrm{m}$ & 0.75 & \\
\hline Reactor depth & $\mathrm{m}$ & 0.03 & \\
\hline Reactor height & $\mathrm{m}$ & 1.5 & \\
\hline $\begin{array}{l}\text { Total vertical illuminated } \\
\text { area }\end{array}$ & $\mathrm{m}^{2} \mathrm{ha}^{-1}$ & 38,500 & both sides of panels included \\
\hline Illumination ratio & & 3.85 & $\begin{array}{l}\text { illuminated reactor surface: reactor } \\
\text { covered ground area }\end{array}$ \\
\hline Volume & $\mathrm{m}^{3} \mathrm{ha}^{-1}$ & 577 & \\
\hline Total surface : volume ratio & $m^{-1}$ & 66.7 & \\
\hline Volume / ground area & $\mathrm{L} \mathrm{m}^{-2}$ & 57.7 & \\
\hline Volume produced per hour & $\mathrm{m}^{3} \mathrm{ha}^{-1} \mathrm{~h}^{-1}$ & 7.21 & 12 hour production day \\
\hline \multicolumn{4}{|l|}{ Process Design } \\
\hline Daily dilution & $\%$ & 15 & \\
\hline Biomass production rate & $\mathrm{kg} \mathrm{m}^{-3} \mathrm{~h}^{-1}$ & 0.025 & $12 \mathrm{~h}$ production day \\
\hline Biomass concentration & $\mathrm{kg} \mathrm{m}^{-3}$ & 2.01 & \\
\hline Air flow rate & $\mathrm{m}^{3} \mathrm{~min}^{-1} \mathrm{~m}^{-3}$ & 1 & Volume air:volume culture \\
\hline $\begin{array}{l}\text { Culture volume processed } \\
\text { per hour }\end{array}$ & $\mathrm{m}^{3} \mathrm{ha}^{-1} \mathrm{~h}^{-1}$ & 3.6 & $24 \mathrm{~h}$ processing day \\
\hline \multicolumn{4}{|l|}{ Major utilities } \\
\hline $\mathrm{CO}_{2}$ requirement & & 1.8 & ton $\mathrm{CO} 2$ / ton biomass produced \\
\hline $\mathrm{CO}_{2}$ net binding in plant & ton $\mathrm{CO}_{2}$ ha $^{-1}$ year & 116 & \\
\hline Polyethylene liner, annual & $\mathrm{m}^{2}$ ha $^{-1}$ year $^{-1}$ & 38,500 & \\
\hline Blower power & $\mathrm{m}^{3} \mathrm{~h}^{-1} \mathrm{~kW}^{-1}$ & 110 & Pressure: 0.15 bar \\
\hline Power for cell harvest & $\mathrm{kWhr} \mathrm{m}^{-3}$ & $0.9-1.1$ & \\
\hline Culture medium premix & ton ha-1 year-1 $^{-1}$ & 63 & \\
\hline Medium filters & cartriges ha-1 & 126 & \\
\hline
\end{tabular}


Table 2-11. Cost of items for flat panel photobioreactor

\begin{tabular}{|c|c|c|c|c|c|c|c|}
\hline \multirow{2}{*}{$\begin{array}{l}\text { Major equipment (MEC) } \\
\text { Scale }\end{array}$} & \multirow[b]{2}{*}{ ha } & \multicolumn{2}{|c|}{ maximum capacity } & \multicolumn{2}{|c|}{ Cost / Unit (€) } & \multicolumn{2}{|c|}{ Number of units ha-1 } \\
\hline & & 1 & 100 & 1 & 100 & 1 & 100 \\
\hline Steel frame & ton ha-1 & \multicolumn{2}{|l|}{88} & 650 & 650 & & \\
\hline Centrifuge Westfalia separator & $m^{3} h^{-1}$ & 22 & 60 & 183,000 & 500,000 & 1 & 0.07 \\
\hline Centrifuge Feed Pump & $m^{3} h^{-1}$ & 12.5 & 80 & 6,000 & 8,000 & 1 & 0.05 \\
\hline Medium Filter Unit & $\mathrm{m}^{3} \mathrm{~h}^{-1}$ & 5.99 & 5.99 & 13,500 & 13,500 & 1 & 0.67 \\
\hline Medium Feed pump & $m^{3} h^{-1}$ & 12.5 & 80 & 6,000 & 8,000 & 1 & 0.05 \\
\hline Medium preparation tank & $\mathrm{m}^{3}$ & 60 & 60 & 20,000 & 20,000 & 1 & 0.72 \\
\hline Harvest broth storage tank & $\mathrm{m}^{3}$ & 60 & 60 & 20,000 & 20,000 & 1 & 0.72 \\
\hline Seawater pump station & $m^{3} h^{-1}$ & 5.99 & 5.99 & 15,000 & 15,000 & 1 & 0.7 \\
\hline Weighing station & & & & 40,000 & 40,000 & 1 & .01 \\
\hline Air blowers & $m^{3} h^{-1}$ & 9,000 & 12,000 & 90,000 & 106,000 & 4 & 0.32 \\
\hline \multicolumn{8}{|l|}{ Operating cost items } \\
\hline Labor & staff ha-1 & 3 & 0.07 & 40,000 & 37,142 & & \\
\hline Payroll charges & $\%$ salary & \multicolumn{2}{|l|}{15} & & & & \\
\hline Maintenance & $\%$ MEC & \multicolumn{2}{|l|}{4} & & & & \\
\hline General plant overheads & $\%$ & \multicolumn{2}{|l|}{55} & & & & \\
\hline \multicolumn{8}{|l|}{ Other fixed costs } \\
\hline Installations costs & $\%$ MEC & \multicolumn{2}{|l|}{30} & & & & \\
\hline Instrumentation and control & $\%$ MEC & \multicolumn{2}{|l|}{10} & & & & \\
\hline Piping & $\%$ MEC & \multicolumn{2}{|l|}{30} & & & & \\
\hline Buildings & $\%$ MEC & \multicolumn{2}{|l|}{30} & & & & \\
\hline $\begin{array}{l}\text { Land (land cost not included as } \\
\text { capital, but interests on land are } \\
\text { included). }\end{array}$ & $€ \mathrm{~m}^{-2}$ & \multicolumn{2}{|l|}{10} & & & & \\
\hline Investment per ha (total land) & $\mathbf{M €}$ & 1.20 & 0.80 & & & & \\
\hline
\end{tabular}




\section{Estimation of photosynthetic efficiency in raceway ponds, horizontal tubular photobioreactors and vertical flat panel photobioreactors.}

\section{Calculation of photosynthetic efficiency}

Methods for calculating photosynthetic efficiency $(\mathrm{PE})$ vary somewhat in practice but $\mathrm{PE}$ can generally be defined as the photobioreactor percentage conversion of the intercepted solar irradiation into biomass-bound energy.

$P E(\%$ solar $)=P_{a}{ }^{*} h^{*} I^{-1} * 100$

Equation 2-7

$\mathrm{Pa}_{\mathrm{a}}$ being areal DW productivity ( $\mathrm{g}$ day ${ }^{-1} \mathrm{~m}^{-2}$, ash free or total $\left.\mathrm{DW}\right) ; \mathrm{h}$ is combustion enthalpy ( $\mathrm{MJ} \mathrm{g}^{-1}$ total or ash free DW), I is intercepted solar irradiation (MJ day ${ }^{-1} \mathrm{~m}^{-2}$ ).

For comparison of different photobioreactor systems, the assessment of the intercepted light is a problematic. The term intercepted may designate surface irradiation of the photobioreactor, integrated over a period of time, thus disregarding surface reflection or it can be an assessment of the light absorption by the culture, which considers the surface reflection from the photobioreactor and absorption in the reactor material. Reflected light from surfaces surrounding the photobioreactor may also play an important role for the calculation of light absorption in vertical systems; normally, the irradiation of a vertical photobioreactor not empirically determined which can be very difficult, but estimated from registered horizontal irradiation data.

The horizontal tubular photobioreactor is a borderline system; with the tubes lying directly on the ground, horizontal solar radiation on the a cross section of the tubes may be used as a measure of intercepted irradiation, but when the tubes are separated and suspended in a cooling water tank with reflective bottom layer, the tubes receive a substantial reflected irradiation from the tank bottom on the lower side and this must be taken into consideration, otherwise, a too low intercepted irradiation is applied and an 
overestimation of the PE results. Irradiation in most cases is measured as photon flux PAR for calculation of solar irradiation based $\mathrm{PE}$, the irradiation in those cases was converted to solar values by dividing with 4.6. PE values indicated as PAR have been converted to solar radiation values by multiplying with 0.42 . $(0.42$ is the average proportion energy content in PAR/total solar spectrum).

The photosynthetic efficiency strongly depend on areal productivity. With respect to the areal productivity data used for the calculations, the figures used are net daily productivities; i.e. including night biomass loss. If only day biomass production figures are used, annual productivity cannot be estimated from the data - night biomass losses of up to $20 \%$ of the daily biomass production is not uncommon.

From papers, where productivity was recorded as a function of different operation procedures, we used the highest productivity results.

The energy content in the biomass is calculated as combustion enthalpy, multiplied with the ash free dry weight biomass produced. Determination of enthalpy is laborious and in most papers, has not been determined for the actual experiment but a previously determined value for the strain is applied. In case of oil accumulation, it could lead to underestimated values, but in general, enthalpy values for a strain vary rather little - as a rule of thumb, less than $5 \%$. We have in several cases calculated PE using combustion enthalpy values for the given strain from other papers.

In some cases, we have calculated PE from areal productivities based on total dry weight an overestimation of PE of in the order of 5-10\% may incur in such cases. There is no bias of the comparison of systems in this aspect.

For the reverse process, - calculating biomass productivity based on estimated photobioreactor PE values and local irradiation, we have used a Roels correlations (Duboc et al. 1999) and an example of basic composition of biomass of Chlorella vulgaris, resulting in a combustion enthalpy value of $26.2 \mathrm{~kJ} \mathrm{~g}^{-1} \mathrm{DW}$. This corresponds to an algal biomass with about 30 \% lipids(Duboc et al. 1999). 
For the open pond and horizontal, tubular reactor, it was assumed that the full irradiation impinging on the surface was absorbed. For the flat panel reactor, light at incidence angles with the panel surface less than 5 degrees, was assumed to be totally reflected and therefore lost. Solrad, version 12 (Pelletier 2002) was used for calculating this fraction

\section{Estimation of $\mathrm{PE}$ in raceway ponds.}

The most widely used microalgal production system is the raceway pond. The raceway pond is a circular channel through which the algal culture is circulation by means of a paddle wheel. The size of industrial raceway ponds vary between 1000 and 5000 $\mathrm{m}^{2}$ (Vonshak et al. 1988). The most characteristic feature of the raceway pond, distinguishing it from the two other systems examined here, the tubular-and the flat panel photobioreactor, is a rather large depth, 10-30 cm(Ben-Amotz et al. 1990, Benemann et al. 1996), a relatively low fluid velocity, typically less than $25 \mathrm{~cm} \mathrm{sec}^{-1}$ in large commercial ponds(Richmond et al. 1986) and a flat, horizontal surface through which the solar irradiation is absorbed and exchange of oxygen with the atmosphere takes place by diffusion. The fact that the culture system is open to the atmosphere does not have a big bearing on the productivity of the system; it carries some limitations with respect to the number of species that are suitable for production in such systems, but in terms of areal productivities and operating costs, another open system, the Czech cascading plate system resembles more closed photobioreactors that raceway ponds.

\section{Validity of productivity data for raceway ponds.}

Productivity in industrial raceway ponds generally is lower than in small experimental ponds (Richmond et al. 1986). In small ponds, productivities up to $40 \mathrm{~g} \mathrm{DW} \mathrm{m}^{-2}$ day $^{-2}$ have been registered with PE (solar) values around 4\% throughout the year (Del Campo et al. 2001). In large scale commercial ponds, annual biomass productivity typically does not exceed 30 ton ha ${ }^{-1}$ year $^{-1}$, which corresponds to an average daily productivity of $10 \mathrm{~g} \mathrm{DW}$ $\mathrm{m}^{-2}$ and a net photosynthetic efficiency of under $1 \%$ (Vonshak et al. 1988).

Direct sources of productivity figures from large commercial ponds is by nature scarce; in an internal report to the US Department of Energy (Benemann et al. 1996), following 
production figures for a number of large industrial Spirulina producers are given: Eathrise farms, 27 ton/ha, Cyanotech 35 ton/ha, Siam Algae, Thailand: 33 ton/ha. On the average, this corresponds to about $10 \mathrm{~g} \mathrm{DW} \mathrm{m}^{-2}$ day $^{-1}$ thus far below the productivity from small experimental ponds.

An extensive review of productivity in microalgal systems was carried out by Goldman in 1979 (Goldman 1979). The conclusion was that long term average productivity figures very rarely extended $20 \mathrm{~g} \mathrm{dw} \mathrm{m}^{-2}$ day $^{-1}$ although short term maximum productivities of $30-35 \mathrm{~g}$ DW $\mathrm{m}^{-2}$ day $^{-1}$ were recorded.

\section{Productivity limiting factors}

Given the apparent contrast between productivity in small and large ponds, it would be useful to know what limits the productivity in large raceway ponds, both for the assessment of the base case PE level and for the increased PE case. Mixing and light integration has been implied (Goldman 1979) but it is likely that mass transfer (oxygen and $\mathrm{CO}_{2}$ ) in many cases is productivity limiting.

Productivity of Spirulina in raceway ponds in Israel was found to be positively related to dissolved oxygen levels (Vonshak et al. 1982) - but at levels under $220 \%$ saturation. However, dissolved oxygen over the entire concentration range was found to limit photosynthesis in Spirulina platensis(Marquez et al. 1995). Mass transfer - removal of dissolved oxygen from the pond to the ambient is poor in raceway pond and may limit the algal productivity (Ugwu et al. 2008). In large commercial raceway ponds, oxygen concentration around noon typically exceeds $400 \%$ saturation(Richmond et al. 1986)and may reach $500 \%$ (Vonshak et al. 1988) but even in small raceway ponds dissolved oxygen levels higher than $30 \mathrm{mg}$ per liter could be measured around noon during summer (Richmond et al. 1990, Jiménez et al. 2003, Moheimani et al. 2007), which is approaching $500 \%$ air saturation values and was verified to limit productivity (Jiménez et al. 2003, Moheimani et al. 2007). When the ponds were heated in the morning, productivity increased.

Mass transfer is proportional with temperature; the liquid diffusion coefficient typically increases with $2.5 \%$ for each degree (Riet et al. 1991) and as the solubility of $\mathrm{O}_{2}$ in water is inversely proportional with temperature, also the driving force for oxygen stripping from 
the pond consequently increases with temperature, which might explain lower dissolved oxygen concentration in one of the cases.

If poor mass transfer and consequently high dissolved oxygen concentrations exerts a practical limiting effect on productivity in raceway ponds, the use of wastewater as nutrient source may thus have a direct beneficial effect on algal productivity by reduction of dissolved oxygen in the pond.

Diffusion of oxygen through the surface was indicated to account for only about $60 \%$ of the removal of oxygen produced in observed raceway ponds (Weissman et al. 1988). It was suggested, that nucleating bubble formation was responsible for the residual oxygen removal and that such bubble formation was observed at dissolved oxygen levels around 3$400 \%$ air saturation. Such oxygen nucleation is not theoretically possible until oxygen saturation exceeds levels in balance with $100 \%$ oxygen atmosphere but one possibility is that the bubbles form around other gas bubbles, for example methane formed in anoxic bottom sediments, and readily diluted by oxygen to a level in balance with the dissolved oxygen in the pond.

We thus hypothesize that mass transfer in many cases limits the productivity in raceway ponds and may explain both why small experimental ponds appear to exhibit higher productivity than large ponds and why use of waste water as nutrient supply may have a positive effect on productivity.

\section{Photosynthetic efficiency in raceway ponds}

For the evaluation of photosynthetic efficiency in raceway ponds, annual productivity measurements along with solar irradiation data and energy content in the produced biomass are required. In addition, the data should be obtained from full-scale raceway ponds or downscaling should be correctly performed as scale has a considerable influence on mass transfer in raceway ponds.

Few long term datasets of productivity in large scale ponds are available; however, in study data cited in (Benemann et al. 1996, Sheehan et al. 1998), the productivity of two $1000 \mathrm{~m}^{2}$ raceway ponds at Richmond, California (near San Francisco) was followed over a period of more than a year. Peak productivities of about $35 \mathrm{~g} \mathrm{DW} \mathrm{m}^{-2} \mathrm{day}^{-1}$ were registered in one 
pond in the months July, August and September. We estimated photosynthetic efficiencies for the two pond time series by assuming a combustion enthalpy of $21 \mathrm{~kJ} \mathrm{~g} \mathrm{DW}^{-1}$ of biomass, which would apply for medium lipid containing algal biomass, and using solar irradiation values for San Francisco for the period, which are available from the USEPA (USEPA 2010). Mean annual photosynthetic efficiency thus calculated, were 1.8 and $2.4 \%$ for the two ponds, the difference due to grazer control by screening in the pond with the high PE (2.4\%). The data are included in Fig. 2-3.

In a raceway pond, built in a greenhouse in Sendai, Japan $\left(38.2^{\circ} \mathrm{N}\right)$, high long-term photosynthetic efficiencies of Chlorella (6.56 \% PAR or $2.8 \%$ solar) were obtained in $0.9 \mathrm{~m}^{2}$ raceway ponds (Hase et al. 2000). The dry weight productivity as well as the photosynthetic efficiency was positively correlated with irradiation in the ponds, which is a an indication the irradiation may not have been the dominant, directly limiting factor as productivity and photosynthetic efficiency are inversely related in light limited systems. The observed relation could be explained by assuming that temperature exerted the primary control on productivity even in the greenhouse for a certain number of the datapoints.

In Fig. 2-3, the photosynthetic efficiency data from various sources are depicted against monthly average daily solar irradiation values in the relevant months. There seems to be no correlation between photosynthetic efficiency and irradiation which may be a result of ponds operating at suboptimal temperatures during months with low irradiation where PE otherwise would be expected to be higher.

\section{Conclusion}

An all-year photosynthetic efficiency of $1.5 \%$ (solar) was adopted as base case PE for raceway ponds, operated with fertilizers as nutrient source. In Fig. 2-3, it appears that there are many points over that limit, but much of the available data are derived from high temperature regions and comprise the use of waste water which for reasons described above would not be realistic for north European sites. A $60 \%$ increase of base case PE that was used appears in the sensitivity analysis as a realistic PE enhancement level is also indicated in Fig. 2-3. 


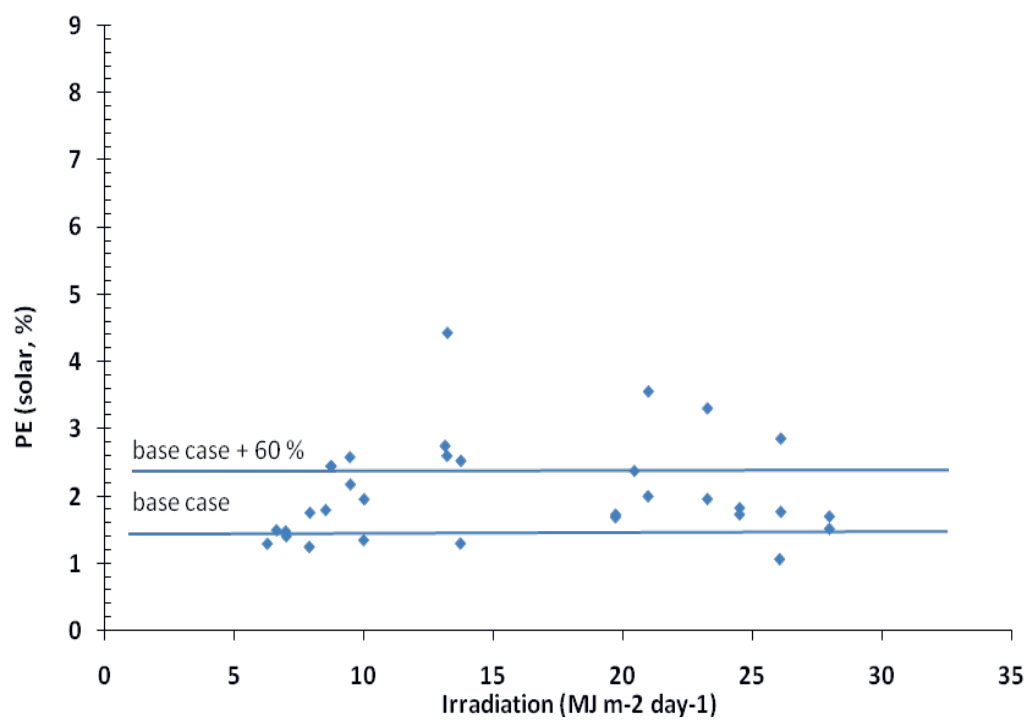

Fig. 2-3. Photosynthetic efficiencies for raceway ponds depicted against average daily irradiation (solar) during the period. Data from (Laws et al. 1983, Pushparaj et al. 1997, Sheehan et al. 1998, Hase et al. 2000, Moreno et al. 2003)

\section{Horizontal tubular reactors.}

Tubular photobioreactors are produced in a number of designs that are variations of the basic principle that mass transfer and photosynthesis are separated. Photosynthesis takes place in transparent tubes through which the culture is pumped and mass transfer (oxygen stripping) takes place in a degasser, a tank in which dissolved oxygen is stripped off, normally by air sparging. The tubular design also means that cleaning of the plant is easy to accomplish at an industrial scale and the tubular photobioreactor is the so-far the only closed photobioreactor type in commercial operation. Commercial plants include the vertical plant in Klöze, Germany, the vertical Salata plant in Ritschenhausen, Germany, the horizontal Algatech plant in Israel, and a number of other tubular plants in Spain, Portugal, Germany and the Netherlands. Different tubes are being applied, most often acrylic or glass due to high transmission, but also disposable soft (polyethylene) tubes are used. In a number of cases, turbulence enhancing devices (bafflers or tube bends) are applied, and may enhance the photosynthetic efficiency of the system. The analyzed data include such examples. 
The turbulence level in the tubes influences the photosynthetic efficiency of the photobioreactor and a number of papers support a directly positive effect of turbulence on productivity up to a threshold where shear damage reduces the productivity.

Residence time in the tubes between each successive pass through the degasser and irradiation determines oxygen build up in the culture and high oxygen levels can reduce productivity and hence photosynthetic efficiency of the photobioreactor; the knowledge of oxygen tolerance of various algal species however, is very limited. An oxygen concentration at the end of the tube $300 \%$ saturation $(670 \mu \mathrm{M})$ has found use as a design criteria for tubular photobioreactors, based on experience with Phaeodactylum and Porphyridium cruentum (Camacho et al. 1999, Acién Fernández et al. 2001), but it should be mentioned that no real optimization of tubular reactors that includes species specific oxygen tolerance has been carried out so-far.

Well designed and operated, tubular reactors can result in very high volumetric productivities 2-3 $\mathrm{g} \mathrm{DW} \mathrm{L}^{-1}$ day $^{-1}$ of at biomass concentrations of 7-9 $\mathrm{g} \mathrm{DW} \mathrm{L}^{-1}$, which is advantageous for the downstream processing, for (Grima et al. 1994, Molina Grima et al. 1994, Molina Grima et al. 1996, Acién Fernández et al. 1998, Carlozzi 2000) which is roughly an order of magnitude higher than may be obtained with raceway ponds, and areal productivities, with area calculated as tube-length multiplied with tube diameter, is generally high, for example summer productivities of 40 - $53 \mathrm{~g} \mathrm{DW} \mathrm{m}^{-2} \mathrm{day}^{-1}$ in southern Spain (Del Campo et al. 2001). However, there is some uncertainty as to how these figures relate to total-land-occupied area, which in practice eliminates the effect of irradiation from below.

The fluid velocity in the tubular reactors is probably the most central parameter since it determines oxygen saturation at the end of the tube, turbulence and shear stress (mainly in the circulation pump); all of which influence the PE of the photobioreactor which is probably why the PE values in Fig. 2-4 are spread over a wider range than the PE values for raceway ponds, despite the reactor in principle offers a much higher degree of control. 


\section{PE in tubular photobioreactors.}

The photosynthetic efficiency has been calculated on the basis of the horizontal irradiation of the area, occupied by the tubes, except for one example,(Acién Fernández et al. 1998) where the absorbed irradiation on tubes suspended in a thermostating bath, was estimated for the entire circumference of the tubes.

In Fig. 2-4, the PE values for horizontal tubular reactors, covering different species and irradiation conditions is depicted against daily irradiation (monthly average) for the relevant production period. The exceptional high value (6.9 \% solar PE) is from a cyanobacteria (Synechococcus aquatilis) culture (Ugwu et al. 2005).

There seems to be no correlation between PE and irradiation level.

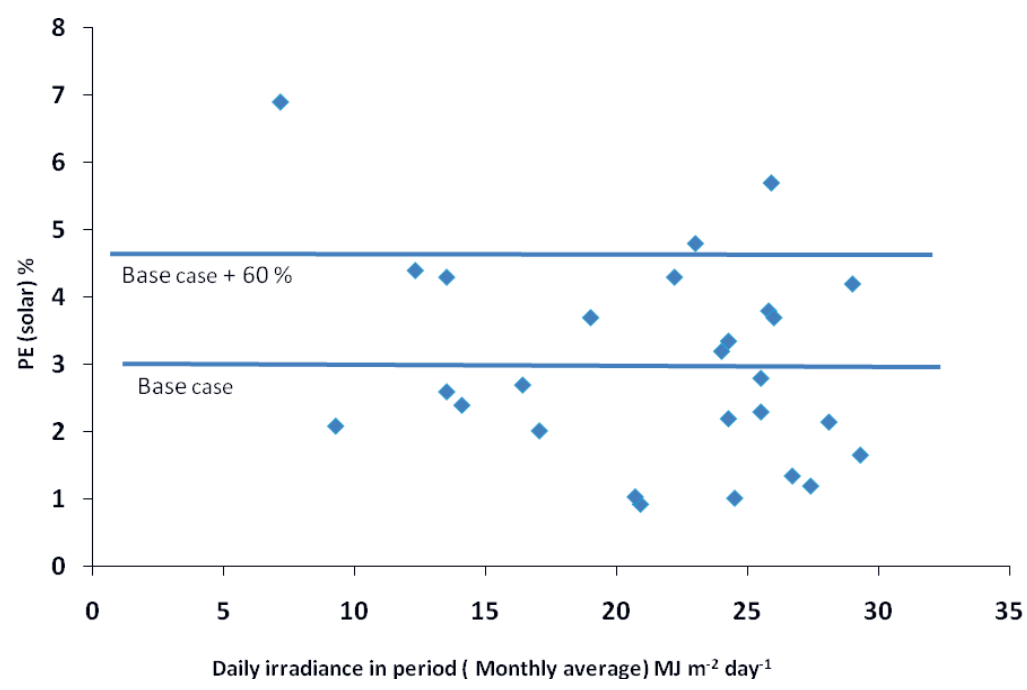

Fig. 2-4 Photosynthetic efficiency depicted against horizontal solar irradiation for a range of horizontal tubular photobioreactors. Data from:(Torzillo et al. 1986, Torzillo et al. 1993, Grima et al. 1994, Molina Grima et al. 1994, Carlozzi et al. 1996, Molina Grima et al. 1997, Acién Fernández et al. 1998, Tredici et al. 1998, Camacho et al. 1999, Carlozzi 2000, Del Campo et al. 2001, Ugwu et al. 2005)

\section{Conclusion}

An all-year base case PE value of $3 \%$ was adopted for horizontal tubular photobioreactors as a base case (lower horizontal line in Fig. 2-4). As for the raceway ponds, base case $+60 \%$ 
encompasses most - but not all of the values and seems therefore a realistic scenario for improvement.

Flat panel photobioreactors.

Productivity

A flat panel photobioreactor is basically a one-compartment, air-sparged aquarium with one of the dimensions - the light path, being relatively short, enabling thereby a large area:volume ratio and options to modulate the irradiation pattern over the day by orienting the panel in various ways towards the sunlight.

Compared with tubular photobioreactors, flat panel photobioreactors are robust and relatively easy to design and operate, but to our knowledge, no commercial or industrial scale flat panel plants have yet bee built. One of the draw backs of the flat panel photobioreactor system, is poor cleanability. Depending on aeration (sparging) rate, the flat panel photobioreactors may have a large mass transfer capability, and very high volumetric or area productivities may be obtained.

With an artificially illuminated short light path $(14 \mathrm{~mm})$ reactors, productivities of 250 and $400 \mathrm{~g} \mathrm{DW} \mathrm{m}^{-2}$ day $^{-1}$ at total irradiation of 2000 and $8000 \mu \mathrm{mols} \mathrm{m}^{-2} \mathrm{sec}^{-1}$, respectively, were obtained(Qiang et al. 1996). PE in those cases were $8.1 \%$ and $2.4 \%$ solar (- equivalent). Applied outdoors in Israel in June with a $2.8 \mathrm{~cm}$ light path flat panel reactor, a productivity of $40 \mathrm{~g} \mathrm{DW} \mathrm{m}^{-2}$ day $^{-1}$ was obtained at a PE of $8.6 \%$ solar (Qiang et al. 1998). Short light path combined with efficient mixing is a prerequisite for utilizing high intensity irradiation at high PE (Hu et al. 1998).

Variations exist - alveolar panels built from extruded dual wall sheet plate (channel plate) and variously structured panels. The channel plate reactors have been built both with horizontal and vertical channels. Another version is the annular reactor which is a "cylindrical panel", formed by the annular space between two concentric cylinders. The effect of varying orientation of the panels was investigated in Israel, and with optimum biomass concentrations of Spirulina, it was observed that with $\mathrm{N}: \mathrm{S}$ orientation of the panels, maximum productivity ensued with angles giving maximum irradiation $(10 \%$ during 
summer and $60 \%$ during winter), whereas maximum PE during the summer, $20 \%$ PAR, approaching the theoretically maximum possible, occurred in vertically oriented panels (Qiang et al. 1998). It was concluded that compared with constantly vertical panel, 26\% additional productivity could be achieved by changing tilt angles twice annually and $35 \%$ by changing tilt angles 4 times per year.

For industrial use of flat panel photobioreactor, we considered only vertical panels, placed at relatively short distances from each other in a N-S orientation, which obviously leads to shadowing between the panels; although it was shown in (Qiang et al. 1998) that it is possible to obtain high photosynthetic efficiency at high irradiance (using short lightpath, about $1 \mathrm{~cm}$ and based on irradiance at the panel surface), it is generally believed that the principle of light dilution obtained through mutual shadowing and reflection between the panels represents the overall best strategy for obtaining high photosynthetic efficiency in the utilization of horizontal irradiation over the entire photobioreactor installation (Richmond 2000). Indeed, a very high productivity (130 $\mathrm{g} \mathrm{DW} \mathrm{m}^{-2}$ day $^{-1}$ ) was reported for a $100 \mathrm{~m}^{2}$ installation with 42 panels in central Germany (Pulz et al. 1998) although the closer circumstances around the work are unclear and border effects are thought to explain the high result. Obviously, consequences of maximizing light dilution through close position of panels are also higher culture volume per area and consequently lower biomass density, higher aeration costs and installation costs and surface cleaning efforts.

\section{Photosynthetic efficiency in vertical flat panels.}

There are not many studies of PE in closely spaced vertical photobioreactors considering the total installation; in Fig. 2-5, we have depicted the few available data against horizontal irradiation. The data also include an annular photobioreactor study where the reactor surfaces produce a similar light dilution although the geometry of the reactor surfaces is different and one single-panel study. Although PE for a photobioreactor installation obviously will be influenced by panel distance, light path and panel height, there is too little data to support any speculations. 
The process layout we have selected for the calculation of flat panel production economy was $1.5 \mathrm{~m}$ high panels in a N-S orientation, with an inter-panel distance of $0.75 \mathrm{~m}$. (half

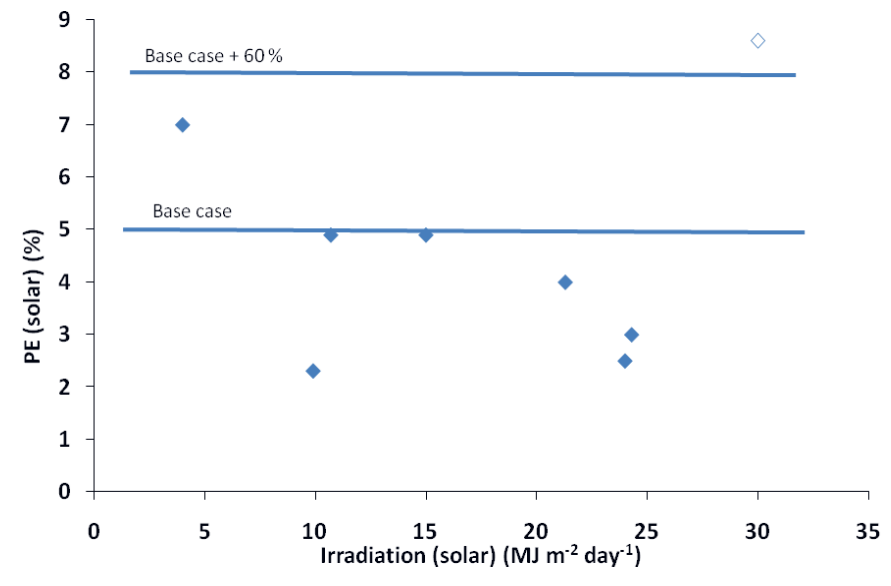

Fig. 2-5. Photosynthetic efficiency depicted against solar horizontal irradiation for a range of flat panel reactor systems. Data from (Tredici et al. 1993, Qiang et al. 1998, Zhang et al. 1999, Zhang et al. 2001, Zhang et al. 2001, Meiser et al. 2004, Chini Zittelli et al. 2006) Closed symbol: PE for entire installation, based on ground surface. Closely spanced Open symbol: PE for single panel, based on panel surface. height). This

distance between

the panels was

selected as the

maximum

distance, which at

the highest solar

elevation in

Netherlands would

ensure that no

direct irradiation

would fall on the

ground between

the panels.

On the basis of the performance of the closely spaced flat panel photobioreactors in Fig.

2-5, an all-year photosynthetic efficiency was assigned.

\section{Conclusion}

An all-year base case PE value of $5 \%$ was adopted for vertical panels as a base case (lower horizontal line in Fig. 2-5). As for the raceway ponds and tubular photobioreactors, the enhanced PE scenario was chosen as base case $+60 \%$. In this case, it encompasses all the values except for the single panel example. The fact that a single panel can be operated at high irradiation with more than $8 \% \mathrm{PE}$, is a strong evidence that it also will be possible for entire installations of vertical panels. Consequently, a PE of $8 \%$ is also a reasonable value for an enhanced PE case. 


\section{On energy ballance and production costs in tubular and flat panel photobioreactors}

Published as: Norsker, N.-H., M. J. Barbosa, M. H. Vermuë and R. H. Wijffels (2012). On Energy Balance and Production Costs in Tubular and Flat Panel Photobioreactors. Technikfolgenabschätzung - Theorie und Praxis 21(1): 54-62

\section{Abstract}

Reducing mixing in both flat panel and tubular photobioreactors can result in apositive net energy balance with state-of-the-art technology and Dutch weather conditions. In the tubular photobioreactor, the net energy balance becomes positive at velocities $<0.3 \mathrm{~m} \mathrm{~s}^{-1}$, at which point the biomass production cost is $3.2 € / \mathrm{kg}$ dry weight. In flat panel reactors, this point is at an air supply rate $<0.25 \mathrm{vol} \mathrm{vol}^{-1} \mathrm{~min}^{-1}$, at which rate the biomass production cost is $2.39 € / \mathrm{kg}$ dry weight. To achieve these values in flat panel reactors, cheap lowpressure blowers must be used, which limits the panel height to a maximum of $0.5 \mathrm{~m}$, and in tubular reactors the tubes must be hydraulically smooth. For tubular reactors, it is important to prevent the formation of wall growth in order to keep the tubes hydraulically smooth. This paper shows how current production costs (2012) and energy requirements could bedecreased. 


\section{Introduction}

Electrical power is used for mechanical mixing in both open and closed photobioreactors and is necessary:

- to keep the algae suspended,

- to provide sufficient mass transfer which denotes the exchange of oxygen and carbon dioxide,

- to obtain a certain level of light integration.

Light integration means shifting the algae between dark and light zones in the light path whereby the resulting productivity increases towards that of a homogenously illuminated culture, illuminated by the time-averaged light intensity. Full light integration implies high frequency of flashing. For the microalga Chlamydomonas reinhardtii, for example, full light integration implies light flashes of only millisecond duration (Vejrazka et al. 2011), which in practical photobioreactors requires a high and energetically costly level of turbulence. Optimizing a photobioreactor in terms of cost or energy is hence a complicated process requiring functional relationships between, on the one hand, oxygen, carbon dioxide and irradiation and, on the other, productivity. Furthermore, it should be kept in mind that these processes are overlaid by daily and seasonal cycles of light and temperature. Until these functional relationships have been developed, optimization remains a trial-and-error process. Some guidance can be obtained by identifying the energy-sensitive parameters in the two cultivation systems. The energy requirements for circulation in the tubular and flat panel reactors are normally given as the specific power supply in watt per $\mathrm{m}^{3}\left(\mathrm{~W} \mathrm{~m}^{-3}\right)$ of reactor volume and is reasonably comparable since the volumetric productivity of the two systems is rather similar.

\section{Power Requirements for Mixing in Photobioreactors}

Many recent (as of 2012) papers have assumed that a specific power supply in the range of 2,000-3,400 $\mathrm{W} \mathrm{m}^{-3}$ is characteristic of tubular photobioreactors (Sierra et al. 2008; Lehr, Posten 2009; Posten 2009; Xu et al. 2009; Jorquera et al. 2010; Morweiser et al. 2010; Brentner et al. 2011; Gilbert et al. 2011; Hulatt, Thomas 2011; Pegallapati, Nirmalakhandan 
2011; Singh, Olsen 2011), but even 6,000 $\mathrm{W} \mathrm{m}^{-3}$ has been assumed to be typical (Brentner et al. 2011). These figures seem to originate from a single experimental study with a small, airlift-driven helical tubular photobioreactor (Hall et al. 2003), in which a power supply of $2,000-3,400 \mathrm{~W} \mathrm{~m}^{-3}$ was given along with a power efficiency of $1-2 \%$. This power calculation is probably not correct, and the value is certainly not characteristic of tubular photobioreactors in general. For example, in a tubular photobioreactor with $6 \mathrm{~cm}$ tubes, a velocity of $1.0 \mathrm{~m} \mathrm{~s}^{-1}$ corresponds to a specific power supply of $170 \mathrm{~W} \mathrm{~m}^{-3}$ (Acién Fernández et al. 2001), and in a recent (as of 2012) paper on a tubular pilot reactor, the actual specific circulation power was about $300 \mathrm{~W} \mathrm{~m}^{-3}$ at a fluid velocity of $0.9 \mathrm{~m} \mathrm{~s}^{-1}$ in $9 \mathrm{~cm}$ (d) tubing (Acién et al. 2012). Burgess and Fernandez-Velasco (2008), using standard hydraulic estimates for smooth tubes at low Reynolds numbers, obtained much lower specific power requirements for circulation. Their calculation, however, did not account for the friction caused by flow elements in the system such as bends, T-pieces and restrictions or for that due to biofilm-induced roughness.

As the power requirement for circulation is very dependent on culture velocity, minimizing velocity will reduce power consumption. But what is the minimum velocity? The velocity is needed partly to create sufficient turbulence for light integration and partly to avoid detrimental concentrations of dissolved oxygen in the tubes (to minimize growth inhibition caused by photorespiration). This inhibitory effect of oxygen can be reduced by establishing a maximum level of dissolved oxygen permitted in the medium. At sub saturating light intensities, Neochloris oleoabundans, for example, had nearly the same growth rate at dissolved oxygen saturation and at three times the saturation level, but at four times the saturation level the growth rates were reduced, although it was possible to reverse the reduction by operating at a high $\mathrm{CO}_{2}$ partial pressure (Sousa et al. 2012). A velocity of $0.5 \mathrm{~m} \mathrm{~s}^{-1}$ was sufficient to keep the oxygen level under $300 \%$ saturation during a passage through a $100 \mathrm{~m}$ tube under high irradiation conditions (Acién Fernández et al. 2001).

A velocity of $0.5 \mathrm{~m} \mathrm{~s}^{-1}$ with Neochloris would thus be a safe, no-oxygen-effect velocity in that system, but the cost-optimized velocity is probably much lower.

With regard to flat panel reactors or bubble columns, the necessary specific aeration power supply can be calculated as the product of the superficial gas velocity, the gravity 
acceleration and the liquid density (Sierra et al. 2008). While the preferred value of $53 \mathrm{~W}$ $\mathrm{m}^{-3}$ emerging from that study has been cited frequently as typical for flat panel reactors, this value does not take the pressure drop over sparger holes or the energy efficiency in the production of compressed air into account and is therefore not a useful indication.

The superficial gas velocity is the most rational basis for discussing mixing in flat panels, but the aeration rate is more relevant in relation to compressor economy (superficial gas velocity is equal to the aeration rate divided by the aerated cross-sectional area of the reactor). Very large differences in the aeration rate are employed in different reactor studies. For small flat plate reactors, an aeration rate of 1 liter of air per liter reactor volume per minute is commonly used (Sierra etal. 2008). Whereas this value may be unnecessarily large for mass transfer purposes alone, high aeration rates normally enhance growth. For example, in Zhang et al. (2002), for a $55 \mathrm{~cm}$ tall flat panel reactor gassed with $10 \% \mathrm{CO}_{2}$, an aeration rate of $0.05 \mathrm{vol} \mathrm{vol}^{-1} \mathrm{~min}^{-1}$ was defined as optimum, although growth increased with aeration rates up to $1 \mathrm{vol} \mathrm{vol}^{-1} \mathrm{~min}^{-1}$. References can also be found to the beneficial effect of applying much larger aeration rates, for example 1-6 vol vol-1 $\mathrm{min}^{-1}$ with high productivity Spirulina culture (Qiang, Richmond 1996). For economic modelling purposes, we have been using an aeration rate of $1 \mathrm{vol} \mathrm{vol}^{-1} \mathrm{~min}^{-1}$ as a base level and consider that reductions down to $0.05 \mathrm{vol}$ vol- $1 \mathrm{~min}^{-1}$ may be optimal in some cases for energy consumption reasons. The energy efficiency of photobioreactors cannot be viewed sensibly without also considering the cost of the mixing. It is important to note that the cost of mixing is composed of power consumption and depreciation of equipment and that the selection of technology (instrumentation) and mixing requirements (process design) can change the magnitude and proportions of the two components considerably.

\section{Mixing Costs in Photobioreactors}

The mixing costs for flat panel reactors consist of the value of the depreciation and cost of the energy consumption of the blowers or compressors delivering the compressed air for sparging, the pressure of which has a dramatic effect on these factors. Roots-type and screw-type blowers produce compressed air at a high energy efficiency over a wide pressure range but are high precision mechanical instruments and very costly. The very commonly used side channel blowers are cheap but energy efficient only at low pressures. 
Radial blowers (the equivalent of a centrifugal liquid pump) are cheap and energy efficient, generally up to higher pressure levels, but this is strongly influenced by scale: large scale radial blowers can be both cheap and energy efficient at higher pressures but also pose an engineering challenge even at a large algal cultivation installation.

The mixing costs for tubular reactors are entirely dominated by the circulation pump costs, which are the sum of the depreciation of the circulation pump and the costs of the pump's energy consumption. It is possible to get widely varying results depending on the process layout and instrumentation, the type of circulation pump being very important to the circulation economy. Large-turbine wheel centrifugal pumps can be very energy efficient but are costly, while small-turbine wheel centrifugal pumps are cheap but less energy efficient and dissipate the energy deficit as shear stress. Airlift pumps have frequently been preferred to avoid possible shear stress damage, but it is necessary to operate them at a large immersion depth to generate the necessary head to circulate the culture. This excludes the use of cheap and energy-efficient, low-pressure blowers. In order to realize the promises of producing cost-efficient microalgal feedstock for biofuel production, it is essential not to spend more energy on agitation than absolutely necessary. In 2011, we published a desk study on the cost of producing microalgal biomass with a base case at a north European site (Netherlands), com-paring three different cultivation methodologies (open ponds, one-layer tubular photobioreactors, and closely spaced flat panel photobioreactors) at a 100 ha scale (Norsker et al. 2011). The current (2011) cost of producing $1 \mathrm{~kg}$ of biomass dry weight (DW) was calculated to be 4.95, 4.15 and $5.96 €$ respectively. These base case results were obtained for a velocity in the tubular reactor of $0.5 \mathrm{~m} \mathrm{~s}^{-1}$ and an aeration rate of $1 \mathrm{vol} \mathrm{vol}^{-1} \mathrm{~min}^{-1}$ for the flat panel reactor. In the tubular reactor, mixing cost constituted $1.27 € / \mathrm{kg}$ DW or $30 \%$ of the total biomass production costs. The cost of the air sparging in flat panel photobioreactor systems was even larger, namely $3.04 €$ per kg DW or $52 \%$ of the biomass production costs. So there is good reason for looking further into the details of these processes and examining if more economical process layouts can be obtained. We therefore believe that a study of energy optimization of closed photobioreactors in the context of classical hydraulics would be useful. 


\section{Economic model}

A techno-economic model was established for producing microalgae under Dutch conditions using open ponds, tubular photobioreactors or flat panel reactors as described in Norsker et al. (2011), and we refer to that paper and the accompanying information for all the details about the calculations. Here, we can briefly state that the microalgal productivity is calculated on the basis of assumptions about reactor-specific typical photosynthetic efficiency (justified in the accompanying information) of ponds, tubular and flat plate photobioreactors. Applying the average monthly irradiation values to different sites then will result in typical biomass productivities for algae with a given caloric value. The individual contributions to the cost of the algal production can then be expressed as the cost per kg algal biomass produced. Here, we perform the calculation with modified input data on mixing costs.

For tubular reactors, a significant amount of energy is required to circulate the culture in the tubes. This energy consumption is necessary to perform the equivalent of mixing in the flat panel reactors and to recycle the culture between the degasser and the solar collector. The energy consumption is usually indicated as the specific power supply, that is the power (in watt) per $\mathrm{m}^{3}$ solar collector. The specific power supply for circulation depends strongly on the culture velocity and the pump efficiency, which are important process design factors. Calculating the power needed for circulating the culture in a circuit (a path from degasser tank through manifold and tube and back again) is done by calculating the power associated with tube wall friction and other power absorbing elements in the circuit separately. The power was calculated using the Darcy-Weisbach equation and estimating the friction factor with the Moody approximation to the Blasius equation, setting the sandequivalent biofilm roughness to either 0 or $1.5 \mathrm{~mm}$ and using an overall (pump + motor) energy efficiency of 0.5 .

In the economic model for the tubularplant, we operated with variably sized reactor units, each provided with one circulation pump with a flow of $1,000 \mathrm{~m}^{3} \mathrm{~h}^{-1}$. In the present case, 100-300 tubes in each direction per pump were necessary. Larger pumps can be found, but units larger than 300 tubes are not practical. The cost of each pump was $29,000 €$. The effect of varying the velocity with tubular reactors and varying the air supply rate with the flat 
panel reactors does not imply any functional relationship between velocity/air supply and the productivity of the systems but is merely an analysis of the cost effect of these parameters.

For the flat panel reactors, the panel height was lower $(0.5 \mathrm{~m})$ than in the previous layout and the distance between reactors was shorter, while the volume of the reactors was kept approximately constant. The height of the panels was set to $0.5 \mathrm{~m}$ as that would be the largest possible height with a total pressure drop over the panels of $100 \mathrm{mbar}$. The purpose of this choice will become obvious later.

In flat panel photobioreactors, compressed air is used for mixing and mass transfer directly in the reactor and constitutes a significant part of the energy consumption. The power supply for sparging depends strongly on the discharge pressure of the compressor, and efficiency as function of the discharge pressure varies strongly between the different types of compressors, so the pressure of the gas used for sparging is an important design factor. It is the sum of the pressure drop over the sparger system and the static height of the reactor. To evaluate the compressor efficiency, it is reasonable to compare it with the energy requirements for isothermal compression, as the compressed air is cooled before injection and the energy represented by compression heat is inevitably lost. For the three actual compressor types for which we managed to acquire data, the total power consumption per volume compressed air delivered was depictedagainst the discharge pressure, but expressed at standard conditions $\left(1 \mathrm{bar}, 20^{\circ} \mathrm{C}\right)$ and compared with the power for isothermal compression.

The data were fitted with various regression formula. The efficiency at a given pressure was directly calculated by dividing the power supply for isothermal compression with the actual requirements of the different compressors.

\section{Results}

\section{Tubular Photobioreactors}

To minimize the mixing costs for tubular photobioreactors, we examined the effect of flow resistance in the tubes as a function of tube wall friction. Fig. 3-1 shows the specific power needed to circulate the algal culture in the tubular photobioreactor plant manifold 
constructions. Tubes $6 \mathrm{~cm}$ in diameter and $100 \mathrm{~m}$ long were used. The specific power was calculated using two different friction factors $(0.025$ for smooth tubes and 0.05 for tubes with an assumed sand-equivalent roughness of $1.5 \mathrm{~mm}$ ). The sand-equivalent roughness of $1.5 \mathrm{~mm}$ is chosen rather arbitrarily, and there is no information available on the hydraulic resistance from biofilm in microalgal bioreactors. Barton et al. (2008), however, estimated that the biofilm sand-equivalent roughness would build up to $2.17 \mathrm{~mm}$ before cleaning in a number of sewers. Yet it is hard to say to what extent this can be seen as a parallel to the situation in a tubular photobioreactor. In comparison, the sand-equivalent roughness of 1.5 $\mathrm{mm}$ is probably a worst-case scenario for tubular photobioreactors. The pump power efficiency was chosen as 0.5 ; with large-turbine wheel pumps, this is probably at the low side.

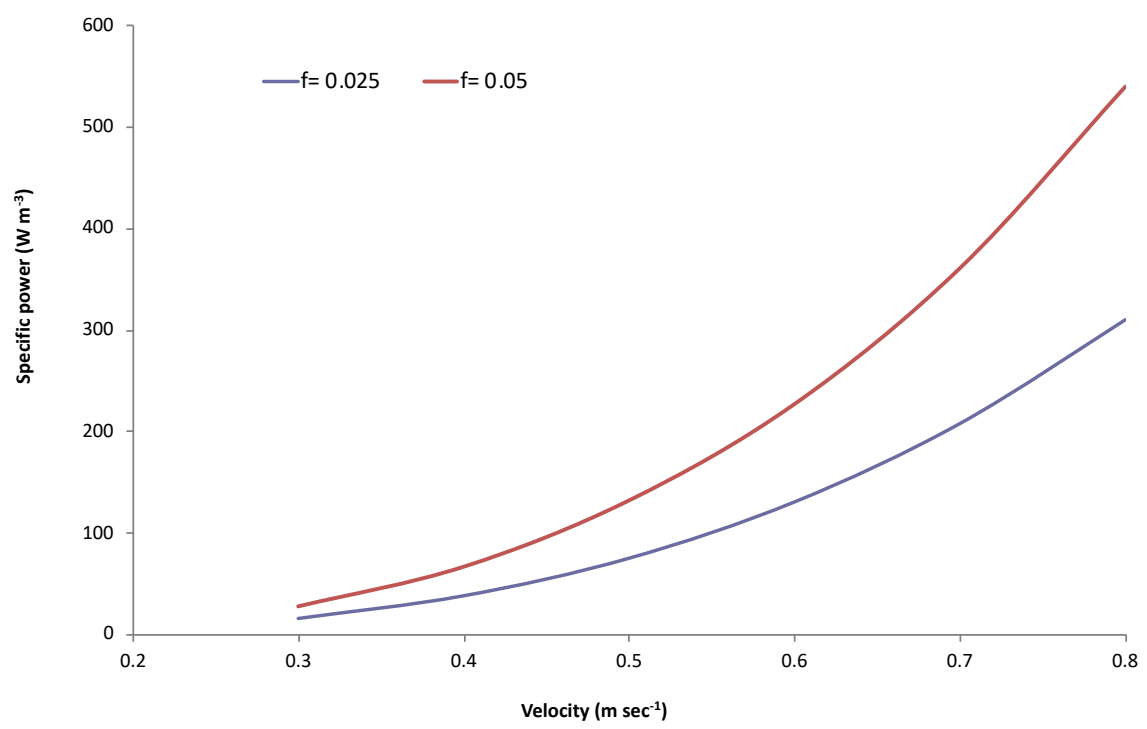

Fig. 3-1 Specific circulation power depicted against tube flow velocity 
Table 3-1 Net energy balance in tubular photobioreactor systems at a velocity of $0.5 \mathrm{~m} \mathrm{~s}^{-1}$

\begin{tabular}{|l|l|}
\hline Energy usage & $\begin{array}{l}\text { MJ kg DW-1algal } \\
\text { biomass }\end{array}$ \\
\hline Pumps & 0.75 \\
\hline Centrifuge & 3.01 \\
\hline Circulation pump & 27.20 \\
\hline $\begin{array}{l}\text { Air blower (degasser) } \\
\text { LDPE 1Y (low density } \\
\text { polyethylene film) }\end{array}$ & 4.40 \\
\hline Energy produced & 9.96 \\
\hline Net balance & 26.20 \\
\hline
\end{tabular}

At a velocity of $0.5 \mathrm{~m} \mathrm{~s}^{-1}$, the resulting specific power is $76 \mathrm{~W} \mathrm{~m}^{-3}$ in smooth tubes and $133 \mathrm{~W}$ $\mathrm{m}^{-3}$ in rough ones.

The net energy balance (the amount of energy produced minus the amount of energy used) per kg DW produced is indicated in Table 3-1 for a velocity of $0.5 \mathrm{~m} \mathrm{~s}^{-1}$. At $0.5 \mathrm{~m} \mathrm{~s}^{-1}$, the circulation pump uses about the same energy as the algae produce.

The effect of velocity on the net energy balance is shown in Fig. 3-2. If the productivity of the culture is maintained at a velocity of about

$0.35 \mathrm{~m} \mathrm{~s}^{-1}$, the energy balance will be positive. Reducing the velocity carries the risk that there will be an increased concentration of dissolved oxygen at the outlet of the loop and that reduced turbulence may reduce the productivity or the desired product formation at a high level of irradiation as a result of the mechanisms mentioned in the introduction, but virtually no empirical information exists to evaluate this effect. It should be kept in mind that the con centration of dissolved oxygen in the tubes is highly dynamic. At constant irradiation, it in-creases linearly over the length of the tubes, and the irradiation is furthermore highly dynamic. In all circumstances, therefore, velocity control would be an important tool for reducing power consumption in tubular reactors. The power supply data were entered in the economic model; the electricity rate used was $5 \mathrm{c}$ per $\mathrm{kWh}$. The number and cost of each pump was selected by varying the number of tubes connected to each pump so that the flow provided by each pump was roughly the same $-1,000 \mathrm{~m}^{3} \mathrm{~h}^{-1}$. At $0.5 \mathrm{~m} \mathrm{~s}^{-1}$, the cost of producing biomass at $\mathrm{f}=0.025$ is $3.97 € / \mathrm{kg}^{-1}$ and at $\mathrm{f}=0.05$ is 4.26 $€ / \mathrm{kg}^{-1}$. At higher velocities, the effect of the friction becomes more pronounced. 


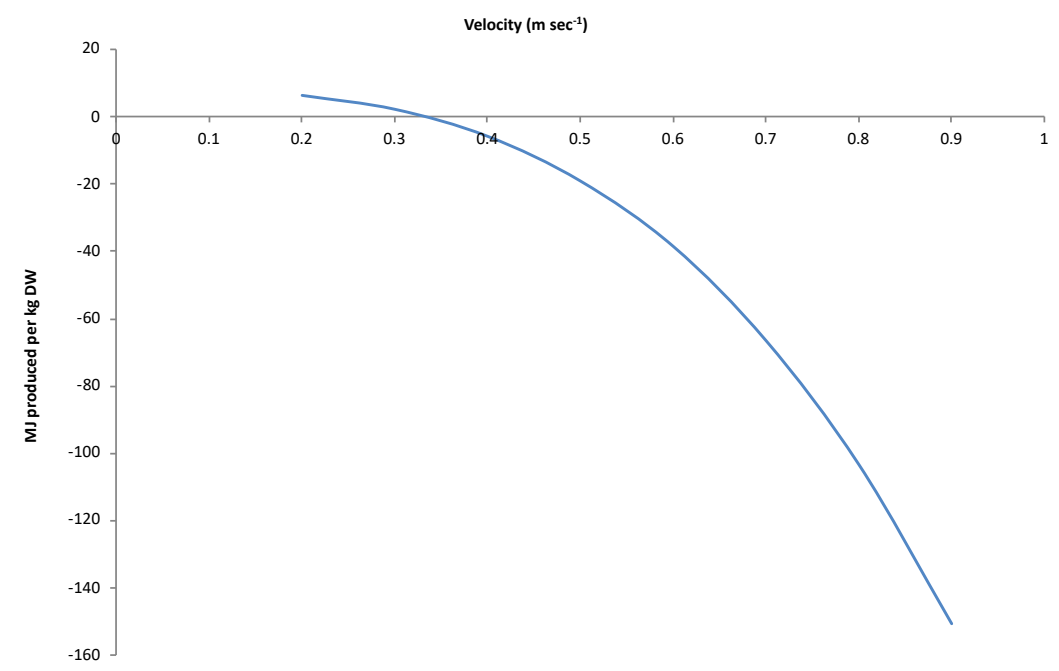

Fig. 3-2 Effect of culture velocity on net energy balance in a tubular photobioreactor

\section{Flat Panel Reactors}

To minimize mixing costs for flat panel reactors, supplier data for power consumption at varying back pressures and flows for three relevant blower types were compared with isothermal compression. The isothermal compression may be considered a theoretical zero-energy loss reference for the blowers as it would imply the compression heat is removed by cooling and not considered conserved in the system.

The energy used by a Roots blower (Omega Industries, model 1012), a side channel blower (Elmo-Rietchle, model 2GH1620) and a radial blower (Elektror, model CFXH 280 B) were also compared. The capacities of these blowers are roughly in the same range (about $10,000 \mathrm{~m}^{3} / \mathrm{h}$ at $\left.100 \mathrm{mbar}\right)$.

They were chosen as suitable to supply the rather large aeration rates of the flat panel reactors. Each blower thus meets the requirements of flat panel reactors occupying a horizontal area of 3,000 $\mathrm{m}^{2}$. Conventional piston compressors are considerably less energy efficient and were à priori not considered. 


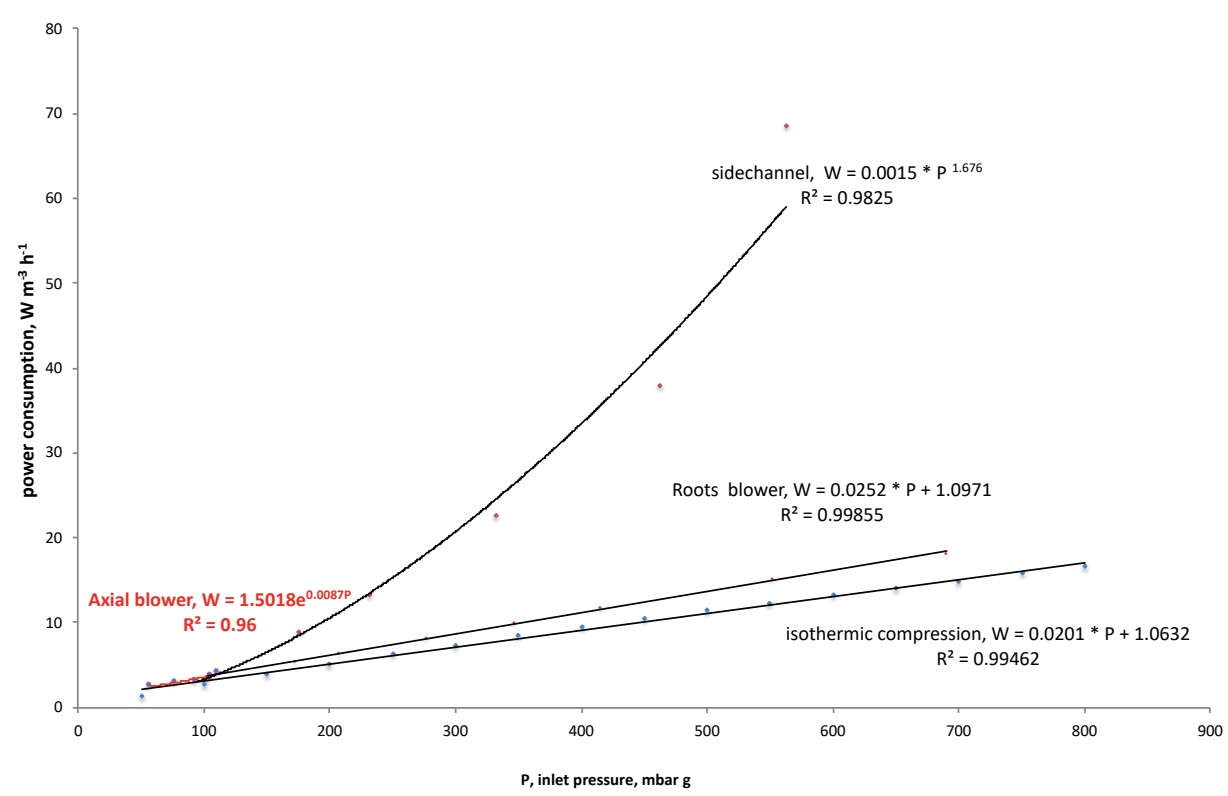

Fig. 3-3 Power supply of different blowers: A Roots blower, a side channel blower, and a radial blower. These three blowers have a similar capacity. The performance is expressed in terms of $k W$ per $m 3$ per $h$ and depicted against discharge pressure. The same representation of the power input for an isothermal compression is included, represented by the black line, allowing a direct evaluation of the performance of the blowers. Note: the axial blower coves only the pressure range indicated by the red curve section.

The power supply per $\mathrm{m}^{3} \mathrm{~h}^{-1}$ flow for the three blowers and for isothermal compression is given in Fig. 3-3 along with regression lines fitted to the data. The Roots blower data were well approximated by a linear regression, and when comparing with the isothermal compression data, the efficiency over the range from 100 to 800 mbar varied from 79.4 to $84.6 \%$. The side channel blower efficiency drops from $80.2 \%$ at $100 \mathrm{mbar}$ to $20 \%$ at 600 mbar. Although no data were available below 100 mbar, it is evident from Fig. 3-3 that if the pressure is below 100 mbar the side channel blower is as efficient as the Roots blower. The last type, the radial blower, has a power efficiency of $82 \%$ at $100 \mathrm{mbar}$, but its pressure range does not exceed 100 mbar.

The blowers were dimensioned to be sufficient for a ground area of 3,000 $\mathrm{m}^{2}$. If, however, it were possible to operate with blowers covering a larger area, it would be possible to obtain a pressure higher than 100 mbar while maintaining high efficiency and low cost. 
The characteristics for the different blower types were incorporated in the economic model, and the resulting total cost per $\mathrm{kg}$ algal biomass produced at an air supply rate of 1 and $0.05 \mathrm{vol} \mathrm{vol}^{-1} \mathrm{~min}^{-1}$ is indicated in Table 3-2. Using a radial blower that is four times larger (CFHX 710) only resulted in marginal savings. The conclusion from Table 3-2 is that if the back pressure from the flat panel reactors is kept below 100 mbar, either radial or side channel blowers can be used with the same result on the economics of production. The

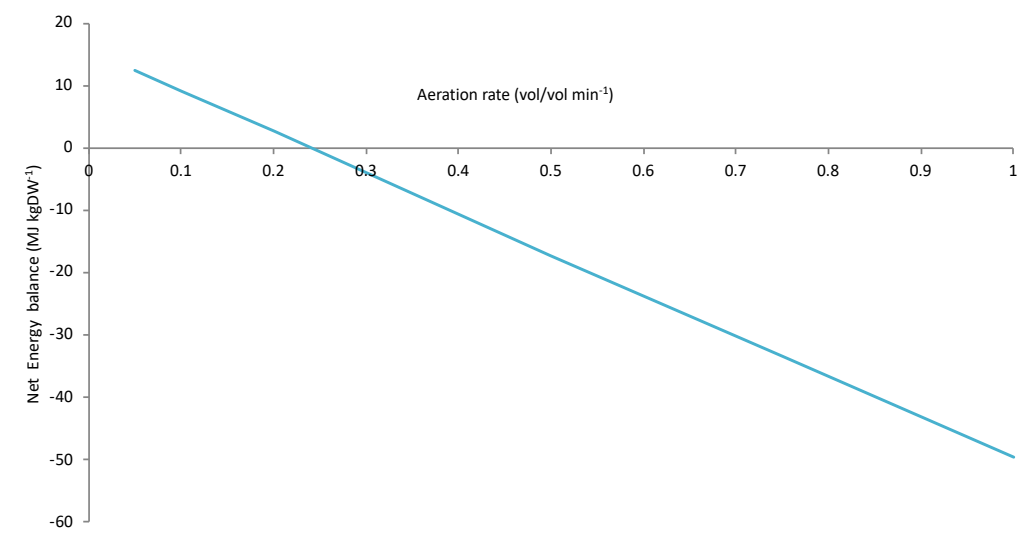

Fig. 3-4 Net energy balance for biomass produced in flat panel reactors (energy produced-energy spent) for Electror CFHX $280 B$ as function of aeration rate at a Dutch site

Roots blower, however, is significantly more expensive due to the high depreciation.

Reducing the aeration rate to the low value of $0.05 \mathrm{vol} \mathrm{vol}^{-1} \mathrm{~min}^{-1}$ as suggested in a number of recent papers (as of 2012) has a significant effect on the economics of production, resulting in biomass production costs of about $2.2 € / \mathrm{kg}^{-1}$ (Table 3-2). The net energy balance is positive at an aeration rate $<0.25 \mathrm{vol} \mathrm{vol}^{-1} \mathrm{~min}^{-1}$, at which point the biomass production cost is $2.4 €$.

Fig. 3-4 demonstrates that a positive energy balance can be obtained for a flat panel reactor if the aeration rate in a low-profile flat panel reactor is limited to $0.25 \mathrm{vol} \mathrm{vol}^{-1} \mathrm{~min}^{-1}$ and the productivity of the reactor is maintained. 
Table 3-2 Calculated cost of algal biomass cultivation as influenced by type of blowe. All blowers operating at full capacity at 100 mbar. Source: The costs for the Roots blower was estimated based on DACE 2002 Microalgae cultivation cost

\begin{tabular}{|l|l|l|}
\hline & \multicolumn{2}{|c|}{$€$ kg DW-1 algal biomass } \\
\hline Air supply rate & $1 \mathrm{vol} \mathrm{vol}^{-1} \mathrm{~min}^{-1}$ & $0.05 \mathrm{vol} \mathrm{vol}^{-1} \mathrm{~min}^{-1}$ \\
\hline Type of blower & & \\
\hline Roots & 4.32 & 2.27 \\
\hline $\begin{array}{l}\text { Radial blower, } \\
\text { CFHX 280 }\end{array}$ & 3.02 & 2.21 \\
\hline $\begin{array}{l}\text { Radial blower, } \\
\text { CFHX 710 }\end{array}$ & 2.99 & 2.18 \\
\hline $\begin{array}{l}\text { Side channel } \\
\text { blower }\end{array}$ & 3.13 & 2.21 \\
\hline
\end{tabular}

\section{Discussion}

In the literature, the value given for the required circulation power of tubular reactors is up to $3,400 \mathrm{~W} \mathrm{~m}^{-3}$, but this high energy demand appears to be based on a misunderstanding of work done on small airlift-driven helical tubular

photobioreactors. The calculations in this paper show that given a suitable design for tubular photobioreactors with smooth tubes, it should be possible to reduce the specific energy demand for microalgae cultivation to $75 \mathrm{~W} \mathrm{~m}^{-3}$ tube volume. Nevertheless, maintaining circulation is still the dominant cost factor in tubular reactors, so it is important to minimize the velocity in tubular reactors. Positive energy balances can result just from reducing the energy spent on mixing in tubular reactors (but also in flat panel reactors). In our calculations, a rather high cost is employed for the pumps, corresponding to the cost associated with slowly turning centrifugal pumps with large impellers which have been selected to avoid shear stress damage, but the worry about shear stress damage of the microalgae in centrifugal pumps is probably out of proportion, and more research into shear stress damage of commercially applicable microalgae is needed. If larger pumps were utilized, pumping costs would also be reduced, but using large pumps also increases the size of each bioreactor unit, which makes contamination more troublesome. In the present case, it was necessary to use 100-300 tubes in each direction connected to a single pump in order to use a $1,000 \mathrm{~m}^{3} \mathrm{~h}^{-1}$ pump. Larger units are not practical. Reducing the flow velocity to the minimum would require looking carefully intothe maximum permissible level of dissolved oxygen, which is an area of microalgal biotechnology that has been investigated very little. The biomass production costs could be reduced below $2 €$ per kg biomass by reducing the velocity to $0.3 \mathrm{~m} \mathrm{~s}^{-1}$ and sourcing a circulation pump for $1,000 \mathrm{~m} 3$ 
$\mathrm{h}^{-1}$ at a head of $0.2 \mathrm{~m} \mathrm{~s}^{-1}$. Further reductions will involve separation technology, operating at more sunny sites, optimizing reactor photosynthetic efficiency, and reducing costs for nutrients and $\mathrm{CO}_{2}$ supply.

In a tubular pilot reactor study, the actual specific circulation energy was about $300 \mathrm{~W} \mathrm{~m}^{-3}$, but a fluid velocity of $0.9 \mathrm{~m} \mathrm{~s}^{-1}$ was used in $9 \mathrm{~cm}$ (d) tubing (Acién et al. 2012).

An average photosynthetic efficiency (solar) of $3.6 \%$ was reported with an equivalent productivity of $90 \mathrm{t} \mathrm{ha}^{-1}$ year $^{-1}$. The actual production costs in the $428 \mathrm{~m}^{2}$ pilot plant were $69.2 € \mathrm{~kg}^{-1}$. A scale up study of the technology was calculated to result in biomass production costs of $12.6 € \mathrm{~kg}^{-1}$, and the authors concluded that a further reduction could be achieved by simplifying technology and materials and by reducing power consumption, manpower and raw materials.

It thus seems that "simple" engineering is the way to achieve significant cost reductions with the tubular system since the photosynthetic efficiency is probably as good as it gets under a natural day cycle. But it should be emphasized that the necessary biological knowledge to do so is still lacking. If, in a flat panel reactor, the pressure drop over the panels is maximum 100 mbar, it is possible to obtain an aeration solution that is economic in both depreciation and power consumption. More than $80 \%$ energy efficiency is readily obtainable with radial and side channel blowers. Direct displacement pumps such as the Roots blowers can produce compressed air very efficiently at higher pressures but are costly and result in high production costs. One possible solution to high profile panels are very large units - with radial blowers - but the channeling and lack of isolation is a serious drawback of this option. If a flat panel reactor has no more than 100 mbar of available sparging pressure, the maximum panel height is probably no more than $50 \mathrm{~cm}$ to allow for pressure drop over sparger holes and in tubing. This raises the problem of air filtration, which probably very few microalgal biotechnologists are prepared to sacrifice.

So what is the solution to this problem? One answer is recirculation of part of the sparger gas, so only a minor part of the sparger gas has to be pressed through filters. Obviously, oxygen levels will rise in the recirculated gas, raising again the question of the maximum amount of dissolved oxygen permitted in photobioreactors. 


\section{Conclusion}

The energy costs for mixing have a strong influence on the economics of microalgal biomass production in photobioreactors, but simple hydrodynamic engineering combined with knowledge of the effect of dynamic oxygen and irradiation conditions on microalgal productivity can potentially turn microalgal photobioreactors into net producers of energy. For tubular photo-bioreactors, this may be accomplished by keep-ing tube wall friction and circulation velocity at a minimum, and for flat panel reactors, by minimizing the aeration rate and using highly energy-efficient blowers. This, in turn, requires that either a low panel profile or very large reactor units be employed, served by single large blowers. 


\title{
4. Productivity of Namnochloropsis
}

oceanica in an industrial closely spaced

\section{flat painel photobioreactor}

Published as: Norsker, N. H., M. Michiels, P. M. Slegers, G. L. A. M. Swinkels, M. J. Barbosa and R. H. Wijffels (2019). Productivity of Nannochloropsis oceanica in an industrial closely spaced flat panel photobioreactor. Algal Research 43: 101632

\begin{abstract}
A growth rate model was established to simulate the growth rate of Nannochloropsis oceanica over a range of values of temperature from 15 to $35^{\circ} \mathrm{C}$ and irradiation from 25 to $205 \mu \mathrm{mol} \mathrm{m} \mathrm{mec}^{-1}$. The model was used to calculate productivity rate with high resolution ( 5 seconds) in an industrial flat panel photobioreactor plant, comprising 20 reactors with a total of 700 individual panels with ambient temperature and horizontal photon flux density as the only input variables. Model predictions were compared with instantaneous productivity data, based on carbon dioxide absorption on 3 selected days with weather extremes: a cloud-less summer day, a summer day with strongly variable cloud cover and a cloudy winter day. Predicted and measured accumulated daytime productivity was 35.4 versus $41.5 \mathrm{~g} \mathrm{dw} \mathrm{m}^{-2}$ panel covered ground area for the cloudless summer day, 27.3 versus $29.7 \mathrm{~g} \mathrm{dw} \mathrm{m}^{-2}$ for the variable-cloud cover summer day and 2.4 versus 1.9 for the cloudy winter day, respectively. Simulated and actual daytime photosynthetic efficiency was to 3-5 $\%$.
\end{abstract}




\section{Introduction}

\section{General}

Design and operation of photobioreactors is a developing discipline. Photobioreactors are closed, transparent vessels used for the production of photosynthetic microorganisms, such as microalgae. Photobioreactors serve both to protect against unwanted organisms and to provide a physical environment that is suitable for photosynthetic production of the cultured species. High biomass productivity and low down-stream processing costs are obtainable with short-light-path photobioreactors. It is possible to obtain short light paths in open reactors; Chlorella sp. was produced outdoors, flowing down an inclined glass surface in a $6 \mathrm{~mm}$ light layer (Doucha et al. 2005) but wider commercial application of this technology does not appear to have taken place. Flat panels offer in addition to short light path and protection against contaminants also the possibility of optimum orientation towards the sun rays and modulation of light intensity through close position (Chini Zittelli et al. 2013). Flat panel reactors may be designed to obtain maximum solar irradiation like photovoltaic panels or the panels may be closely spaced to obtain so-called light dilution: a larger light absorption area and surface reflection between panels reduces the average light intensity which may result in a higher photosynthetic efficiency. The current work focuses on the ProviApt: an industrial photobioreactor, produced by Proviron Industries, Belgium in a fully automated production process.

The ProviApt reactor system has the potential to become a low-cost photobioreactor with a high degree of culture integrity protection. Furthermore, the instrumentation costs are low because process control is exerted in parallel on 2 levels: panels are operated in parallel within one reactor and reactors are operated in parallel within one control unit. Further description of the system can be found elsewhere (Posten et al. 2012, Chini Zittelli et al. 2013, Enzing et al. 2014) 


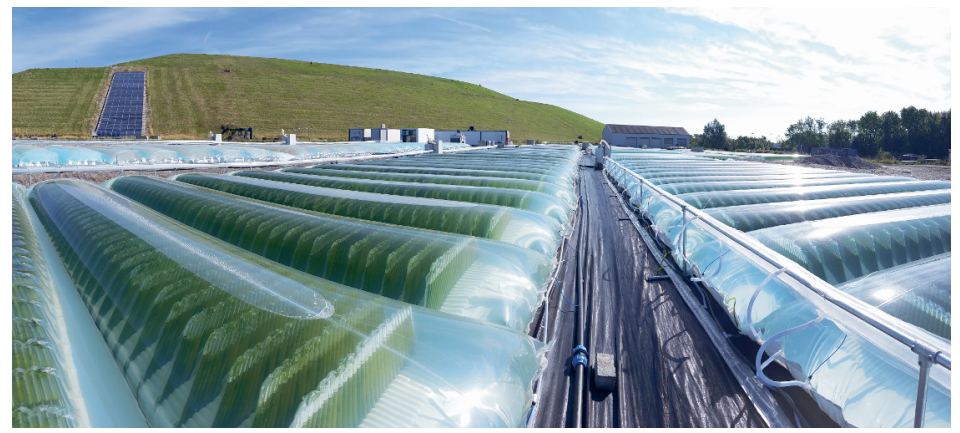

Fig. 4-1 Proviron plant in Antwerp 2015, comprising 120 reactors with 4 control units, each operating 30 reactors in parallel. A feed container in turn services multiple units. The first unit with 20 reactors was operating through 2012

\section{Design}

The 35 panels of the ProviApt photobioreactor constitute a culture volume of $0.175 \mathrm{~m}^{3}$ and are suspended by buoyancy in a water-filled outer bag (Fig. 4-1 and Fig. 4-2). The water in the outer bag is referred to as buffer water. Panels and outer bag are made from transparent polypropylene ( $90 \%$ transparency) film while the bottom is made from a highly reflective white polypropylene film with $90 \%$ light reflectance, adding considerably to the total light flow impinging on the photobioreactor.

The footprint of the outer bag is $8 \times 1.5 \mathrm{~m}$ and it has a water volume of $7 \mathrm{~m}^{3}$. On either side of the panels, a strip of $0.25 \mathrm{~m}$ is left void for practical reasons. Each panel measures $1 \mathrm{x}$ $0.5 \mathrm{~m}$ and has an average thickness (lightpath) of $0.01 \mathrm{~m}$. The distance between the panels in the reactor is $0.2 \mathrm{~m}$.

The ratio between panel covered area and total footprint area of the reactor is thus $35 \mathrm{x}$ $0.2 /(8 \times 1.5)=58 \%$. For area based productivity calculations, the panel covered area is used.

The panels (Fig. 4-2) are divided in 36 tubes by vertical welding seams; in total, the welding seams occupy $10 \%$ of the width of the panel. Each tube is sparged through a sparger hole at the bottom from an aeration layer driven by a pressure difference of 120 mbar over perforations. The $120 \mathrm{mbar}$ thus constitutes static height of $50 \mathrm{~cm}$ and a pressure drop over the perforations of 70 mbar. 


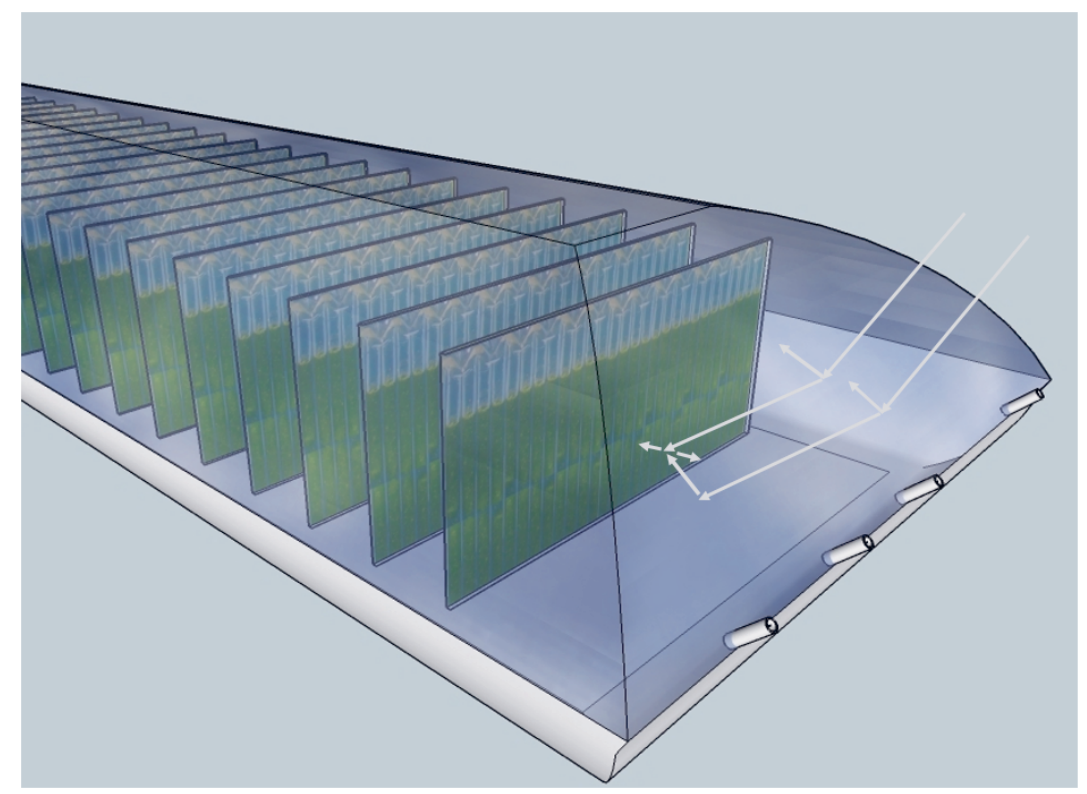

Fig. 4-2 Drawing of the ProviApt photobioreactor, showing the path of light rays, impinging on a unit panel surface: a light ray is partially reflected, partially refracted at the entrance through the outer bag, according to Fresnel's law at an air:water boundary.

The water filled outer bag also functions as a temperature buffer, reducing the requirement for cooling by dissipating accumulated heat during night. In the individual reactor, the 35 panels provide a rapid leveling of the reactor temperature, both vertically and longitudinally (i.e., between panels).

The 20 reactors are operated in parallel by a common controller unit, that feeds growth medium to the reactors, recycles sparging gas, feeds $\mathrm{CO}_{2}$-enriched, ambient air, distributes compressed air for valve operation, provides buffer water circulation and heating and PLC process control. The controller units in turn are operated by an operator station that contains operator interface, medium make-up and harvest unit for primary dewatering of the harvest.

The PLC controller registers 5-second interval values of:

- horizontal irradiance

- temperature of mixed, buffer water outlet

- inlet flow of ambient air to the system

- recirculation flow of gas in the system 
- $\mathrm{CO}_{2}$ inlet flow into the system

- measured $\mathrm{CO}_{2}$ concentration in the recirculating gas

- gas pressure in the panel headspace

- gas pressure at the inlet to the panels

- buffer water pressure for measuring filling height

With these parameters, the $\mathrm{CO}_{2}$-consumption/uptake per $\mathrm{m}^{2}$ of panel covered reactor area and carbon-based productivity may be calculated and biomass productivity may be estimated with a given C:DW ratio.

\subsection{Organization of the laboratory research}

collaborated on testing and developing the reactor system. As a tool in that work, a productivity model for the reactor was developed, depending on only two measured variables: horizontal irradiance and reactor temperature. The model was validated with empirical productivity results from an industrial scale installation, consisting of 20 reactors, operated by Proviron in Belgium.

There are many parameters implicated in the optimization of the productivity of the reactor, including geographical position of the reactor, atmospheric clarity, temperature, panel spacing, orientation of the reactor, panel height, and biomass density (Slegers et al. 2011). Empirical testing of these relations is cumbersome and overlaid by variability in irradiation and operational disturbances. A computer model that simulates the productivity of the reactor, using readily available data would be a valuable tool in optimizing the reactor system. 
A central element of the productivity modeling is the specific growth rate model (Fig. 4-3) that calculates the algal instantaneous growth rate as a function of ambient irradiance and reactor temperature. We selected a growth rate model from literature and fitted the model to steady state dilution rates, measured in a continuously illuminated laboratory

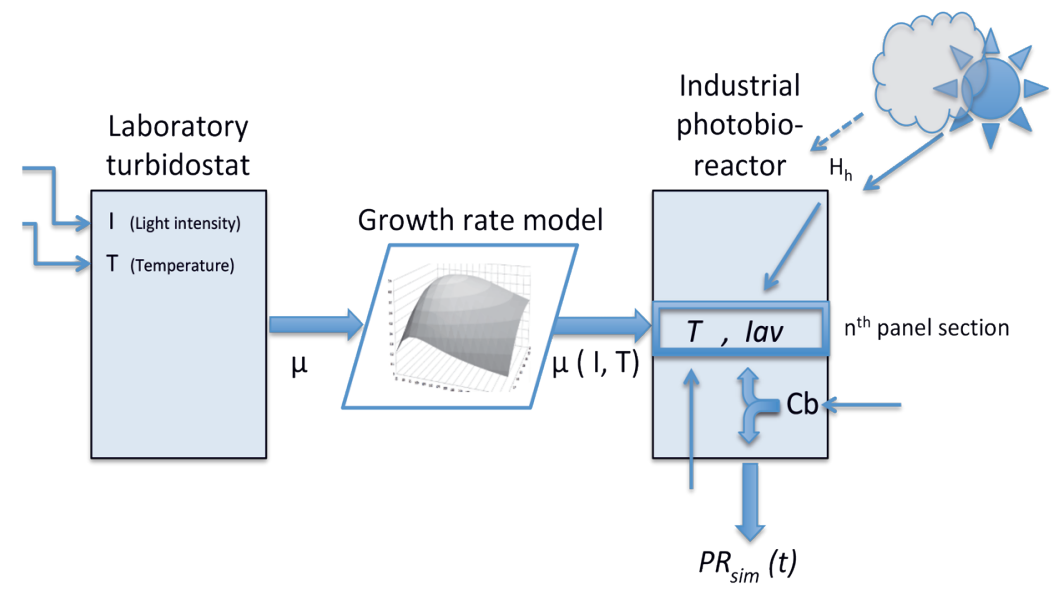

Fig. 4-3 Simulation of the productivity rate in the industrial photobioreactor. A growth rate model is made as a function of light intensity and temperature. With the industrial photobioreactor, the growth rate model is applied to reactor tempertature and average light intensity in each of the vertical panel sections to calculate the productivity rate $\left(P R_{\text {sim }}(t)\right)$.

turbidostat.

The turbidostat was operated at low biomass density to avoid steep light gradients in the turbidostat. The turbidostat was operated under continuous irradiation and covering the full feasible light intensity range from 25 to $205 \mu \mathrm{mol} \mathrm{sec}^{-1} \mathrm{~m}^{-2}$. At an irradiation below that range, the dilution rate became difficult to measure and above the range, the steady state tended to become unstable. Following the nomenclature of Béchet et al. (Béchet et al. 2013), a model depending on two parameters such as light intensity and temperature may be named coupled if one parameter modifies the influence of the other parameter on the growth rate, but uncoupled if the two parameters affects the growthrate in independent manners. Coupled models generally depend on more coefficients than uncoupled models. Type I models depend on one parameter (i.e. light), type II models depend on two (+)parameters and type III models furthermore consider the time history of the algal culture in a dynamic manner. Due to the complexity of type III and coupled type II models they tend to involve many coefficients and hence be difficult to fit. For engineering 
assessments of full scale systems, such as the current situation, Béchet et al. therefore recommend the use of uncoupled, type II models. In the current work we were limited by a very low number (16) of observations and accordingly selected an uncoupled, type II model. The second important element is the light model. The light model converts horizontal, ambient irradiation to irradiation, impinging on the panel surface. The output of the light absorption model is a vertical light distribution on the panels, whereas the panels are considered homogeneously illuminated across the width. The light model was done using ray tracing which is a method for calculating the path of waves or particles through a system with regions of varying propagation velocity, absorption characteristics, and reflecting surfaces. Under these circumstances, wave fronts may bend, change direction, or reflect off surfaces, complicating analysis. Ray tracing solves the problem by repeatedly advancing idealized narrow beams called rays through the medium by discrete amounts. Solar radiation is simulated by propagating many rays with different angles of incidence and wavelengths. When applied to electromagnetic radiation, ray tracing often relies on approximate solutions to Maxwell's equations that are valid as long as the light waves propagate through and around objects whose dimensions are much greater than the light's wavelength, which is the case for the algae reactor. Ray theory does not describe phenomena such as interference and diffraction, which require wave theory (involving the phase of the wave). The calculations of the vertical light profile over the reactor panels were carried out with the ray tracing model RAYPRO. This model was developed by Wageningen UR Greenhouse Horticulture and has been used and validated in several research projects in the field of greenhouse covering materials (Swinkels et al. 2001), microstructures for PV cells (Sonneveld et al. 2005) and solar systems (Sonneveld et al. 2010). Direct solar radiation is simulated as a large number of perpendicular rays. Diffuse light (attaining significant proportion of the light during cloudy conditions) is simulated as randomly directed equally spaced rays in direction cosine space (Lambertian). From the light source, a large number of rays are traced through the object until the ray's intensity drops below a certain threshold value.

The third important element is the actual light intensity to use with the growth rate calculation in the industrial photobioreactor. In a photobioreactor panel with high biomass concentration, there are two different approaches to light intensity assessment: the local 
light intensity method in which local productivity is calculated in steps along the light path or the average light intensity, where the productivity for the entire light path is calculated with the integrated version of the Lambert-Beer formula. The difference between the methods is related to the influence of mixing on light utilization and one is not generally more accurate than the other. The methods were compared in (Bosma et al. 2007). In the current work, the average light intensity method was used.

The biomass productivity rate, expressed in $\mathrm{g} \mathrm{dw} \mathrm{m}^{-2} \mathrm{hr}^{-1}$, was calculated on the basis of simulated growth rate and biomass concentration and was validated with productivity rate values, obtained from $\mathrm{CO}_{2}$-consumption/uptake for the photobioreactor.

\section{Materials and methods.}

\section{Strain and propagation.}

The strain used for both laboratory work and in the ProviApt reactor was Nannochloropsis sp. (CCAP 211/78), now assigned to species Nannochloropsis oceanica (Fawley et al. 2015). Axenic Nannochloropsis was propagated in $250 \mathrm{~mL}$. conical flasks with $100 \mathrm{~mL}$ culture on an orbital shaker and irradiated from above with an approximate photon flux density of 25 $\mu \mathrm{mol} \mathrm{m} \mathrm{sec}^{-1}$ and at a temperature of $20^{\circ} \mathrm{C}$. Prior to inoculation of the laboratory turbidostat reactor, the axenic state of the culture was verified on standard bacterial plate count agar in microalgal growth medium.

Purity of the cultures was verified before used and occasionally during operation of the reactor and no bacterial contaminations were observed in the cultures that were used in the work.

\section{Cultivation medium}

The cultivation medium for the laboratory turbidostat reactor experiments was an artificial sea water medium based on (Zou et al. 1999) with following modifications: phosphate concentration was raised 3.7 times to avoid phosphate deficiency; resulting N:P ratio was 20. Hydrogen carbonate addition was raised 4 times to ensure sufficient carbon during start-up of the culture until the $\mathrm{pH}$ increase, produced by growth would trigger $\mathrm{pH}$ controlled $\mathrm{CO}_{2}$ addition. Vitamins were included in the axenic laboratory turbidostat cultivations as a precaution to standardize growth conditions: Thiamin: $1000 \mu \mathrm{L}^{-1}$, Biotin: 
$200 \mu \mathrm{gL}^{-1}$ and $\mathrm{B}_{12}: 50 \mu \mathrm{gL}^{-1}$. Vitamins were not added in the industrial microalgal production for economical reasons. Nannochloropsis for example does not depend on external vitamin $\mathrm{B}_{12}$ but has several enzymes that might operate faster with additional $\mathrm{B}_{12}$ (Jinkerson et al. 2013).

Biomass yield on nitrogen at nitrogen depletion was $6.1(\mathrm{w} / \mathrm{w})$ in batch cultivation (Wan et al. 2013). The medium used in the laboratory turbidostat experiments contained $0.2 \mathrm{~g}$ nitrogen $\mathrm{L}^{-1}$ which is hence sufficient for nitrogen replete growth up to a biomass concentration of about $1.2 \mathrm{~g} \mathrm{DW} \mathrm{L}^{-1}$. The turbidostat at set point operated at a biomass concentration around $0.1 \mathrm{~g} \mathrm{~L}^{-1}$ (see Table 4-2) so the turbidostat measurements can be assumed to have been conducted under nutrient replete conditions.

The medium was sterile filtered into a 12 liter Nalgene bottle, which was sterilized together with the reactor vessel. The medium was prone to precipitation in the Nalgene bottle; to prevent or reverse this, the Nalgene bottle headspace was pressurized slightly with compressed $\mathrm{CO}_{2}$.

The cultivation medium for the industrial photobioreactor was produced from stock solutions by an automated mixing station. The resulting salinity was 27 ppt. Natural seawater was not used for reasons of convenience. Nitrogen source was $\mathrm{NaNO}_{3}$ rather than $\mathrm{KNO}_{3}$ as in the laboratory turbidostat medium for cost reasons (potassium was provided with the phosphate).

Element composition of the industrial photobioreactor medium and the laboratory turbidostat medium is compared in Table 4-1.

For both laboratory turbidostat and industrial photobioreactor, the media used for inoculum or startercultures were identical to the media used in the final stage. 
Table 4-1 Selected elements in the cultivation medium used for the industrial photobioreactor and the laboratory turbidostat. Note: Fe was provided as FeEDDHA (ferric-ethylenediamine di-2hydroxyphenyl acetate) in the industrial photobioreactor medium and as FeEDTA (ferricethylenediamine tetraacetic acid) in the laboratory turbidostat medium.

\begin{tabular}{|l|l|l|l|}
\hline Element & $\begin{array}{l}\text { Industrial photo- } \\
\text { bioreactor }\end{array}$ & $\begin{array}{l}\text { Laboratory } \\
\text { turbidostat }\end{array}$ & unit \\
\hline $\mathbf{N}$ & 25 & 14.34 & $\mathrm{mM}$ \\
\hline $\mathbf{P}$ & 0.85 & 0.72 & $\mathrm{mM}$ \\
\hline $\mathbf{C}_{\mathbf{i}}$ & 0.24 & 0.38 & $\mathrm{mM}$ \\
\hline $\mathbf{C a}$ & 3.40 & 10.20 & $\mathrm{mM}$ \\
\hline $\mathbf{F e}$ & 60 & 3.90 & $\mu \mathrm{m}$ \\
\hline $\mathbf{M n}$ & 10.4 & 0.45 & $\mu \mathrm{m}$ \\
\hline $\mathbf{Z n}$ & 2.3 & 0.04 & $\mu \mathrm{m}$ \\
\hline $\mathbf{C o}$ & 0.24 & 0.02 & $\mu \mathrm{m}$ \\
\hline $\mathbf{C u}$ & 0.1 & 0.02 & $\mu \mathrm{m}$ \\
\hline $\mathbf{M o}$ & $\mathbf{1 . 0}$ & $\mathbf{0 . 1 2}$ & $\boldsymbol{\mu m}$ \\
\hline & & & \\
\hline
\end{tabular}

\section{Laboratory turbidostat reactor}

The laboratory turbidostat reactor was a FMT 150 reactor system from Photon systems. The reactor vessel (Photonsystems 2018) consists of a frame of stainless steel with the two sides covered by $3.3 \mathrm{~mm}$ flame proof glass. Temperature control was achieved through the steel frame with Peltier elements. The reactor chamber width was $10 \mathrm{~cm}$ and culture depth $2.5 \mathrm{~cm}$ and culture height kept at $16 \mathrm{~cm}$, resulting in a culture volume of $400 \mathrm{~mL}$. The laboratory turbidostat reactor was fitted with an Ingold combination electrode for $\mathrm{pH}$ measurement. The experimental reactor was sparged with air from a central compressed air supply (dry, oil free), regulated by a mass flow controller, set to a fixed rate of 0.4 liter per minute. After the mass flow controller, the air was bubbled through a moisturing flask and entered the reactor through a hydrophobic $0.22 \mu \mathrm{m}$ filter. Carbon dioxide was added to the spargeing air with a mass flow controller, manually adjusted through each time series to keep $\mathrm{pH}$ around 8.0.

The outlet from the reactor was collected in a conical flask, placed in a dark icebox to prevent further growth in the samples until analysis (once daily).

The FMT 150 reactor was operated in turbidostatic mode with target $\mathrm{OD}_{680}=0.4$. (Optical density at a light path of $2.3 \mathrm{~cm}$ ). 


\section{Irradiation of the laboratory turbidostat}

The illumination was a field of 8 by 12 , red $(630 \mathrm{~nm}$ ) LED lamps, illuminating the reactor vessel from the side. The opposite side was covered with a black plate, protecting the reactor from ambient light. The light field was not even with this reactor - a description of the light field can be found in the annex in (Vejrazka et al. 2011). Photon flux density was therefore measured in 5 replicas, scanning over the back side (outside) of the cuvette with the integrating function of a Licor Li-250 A meter with a $2 \pi$ PAR sensor. The measurement was done with growth medium with air sparging in the reactor. The measured photon flux density was a little lower (typically about 5\%) at the back with air spargeing on as opposed to with no air spargeing. This was believed to be caused by scattering of light entering the reactor by the air bubbles.

Biomass concentration remained rather uniform around $0.1 \mathrm{~g} \mathrm{DW} \mathrm{L}^{-1}$. At that biomass level, light intensity at the back side of the reactor (outlet) was around $80 \%$ of inlet $\left(I_{0}\right)$ intensity. The use of red light in the laboratory turbidostat as opposed to daylight, was not per se considered a problem for the translation of growth rate results to daylight photobioreactor conditions. Light color, however, may influence the productivity in a panel, operated at high biomass concentration and strong irradiation. Yellow light, for example, is less readily absorbed by the algae than red and blue light and penetrates more deeply into the reactor and can result in higher productivity (de Mooij et al. 2016). But as there were no steep light gradients in the current turbidostat cultures, the use of day light would therefore have led to similar results as red light.

\section{Dilution rate.}

Overflow from the laboratory turbidostat reactor was collected in a sterilized sample flask and weighed on a daily basis. During the collection of the sample, the flask was kept dark in an ice-box to minimize physiological activity in the algal sample; temperature in the flask varied between 0 and $10^{\circ} \mathrm{C}$.

Dilution rate was calculated as:

$D=\frac{\Delta L}{\Delta t * V}\left[d^{-1}\right]$ (Equation 4-1) 
$\Delta L=$ culture volume collected during $\Delta t, \Delta t=$ sampling time (days) and $V$ being the volume of culture in the reactor. One dilution rate value per day was thus obtained.

\section{Steady state definition.}

The reactor was considered having reached a steady state when the daily dilution rate appeared constant, i.e., the trend exhibited no obvious slope. To record a steady state set of values, sampling (for dilution rate, biomass, total fatty acid content and externally measured, optical density) then continued for a minimum of 4 days. The ratio of optical density at $680 \mathrm{~nm}\left(\mathrm{OD}_{680}\right)$ to biomass concentration should remain constant during the steady state and was followed to monitor the stability of the steady state.

Decreasing OD/biomass ratio is an indication that algal cells are aggregating or increasing their cell volume and is caused by a decrease in the turbidity of algal suspension: The turbidity of a cell suspension is given by:

$\tau=N \pi \frac{d^{2}}{4} Q(d, \lambda)$ (Equation 4-2)

(Masy et al. 1995).

The turbidity is directly proportional with the square of a cell size (diameter, $d$ ) while the cell number, $\mathrm{N}$ is inversely proportional with the cube of the cell diameter when the total cell volume and biomass is constant. $Q$ is a scattering constant.

\section{Chemical analyses}

Biomass was determined on pre-dried, pre-weighed GF/F filters, washed after filtration with $0.5 \mathrm{M}$ ammonium formate and dried at $100^{\circ} \mathrm{C}$ and weighed on a 5-decimal balance. 3 filters were used per measurement.

Total fatty acids (TFA) were determined using a Bligh and Dyer protocol (Breuer et al. 2013) and identified fatty acids were added to calculate TFA in percent bomass dry weight.

\section{Quantum yield.}

The light adapted quantum yield, $\left(\frac{F^{\prime} m^{-}-F_{0}^{\prime}}{F^{\prime} m}\right)$ measurement facility of the FMT 150 was applied. Measurement was carried out with 10 minute intervals and averaged over each steady state series. At light intensity levels over $100 \mu \mathrm{mol} \mathrm{m} \mathrm{mec}^{-1}$, it became increasingly difficult to obtain sustained fluorescence readings resulting in many 0 -values in the log files 
while the remaining readings were consistent. 0-readings consequently were filtered out of the files before averaging.

\section{Operation of the industrial photobioreactor}

The plant was operated semicontinuously, harvesting at the end of the day every 2-3 days and replenishing with fresh cultivation medium. The volume harvested was set to keep biomass concentration around $3 \mathrm{~g} \mathrm{dw} \mathrm{L}^{-1}$ during the summer and $2 \mathrm{~g} \mathrm{dw} \mathrm{L} \mathrm{L}^{-1}$ during the winter. Algal samples for dry weight measurement were taken from the harvest tank. Sparging gas was recirculated in the plant at a rate of 5-10 $\mathrm{L} \mathrm{min}^{-1} \mathrm{~m}^{-2}$ (panel covered ground area) or $0.2-0.4 \mathrm{~L} \mathrm{~min}^{-1} \mathrm{~L}^{-1}$ (panel volume). Carbon dioxide concentration in the recirculating sparging gas was maintained at $0.3 \%$.

$\mathrm{pH}$ was measured off-line once daily. $\mathrm{pH}$ was not directly controlled but varied during the current work between 7.0 and 7.4, reflecting the $\mathrm{CO}_{2}$ concentration maintained in the recirculating gas.

Temperature was not controlled during the summer period, but varied sinusoidaly over the day with minimum in the morning and maximum in the evening; on 09.09.2012, a clear-sky summerday with an irradiation of $21.08 \mathrm{MJ} \mathrm{m}^{-2}$ for example, from 23 to $28 \mathrm{deg}$. In the winter, waste heat was used to heat the buffer water to $20 \mathrm{deg}$.

\section{Productivity modelling}

\section{Overview}

Growth rate distribution over the height of the panels in the ProviApt reactor was modelled according to the flow diagram in Fig. 4-4. The 4 main sections of the model (light model, calculation of panel light intensity, growth rate model and panel productivity rate) are commented below. 


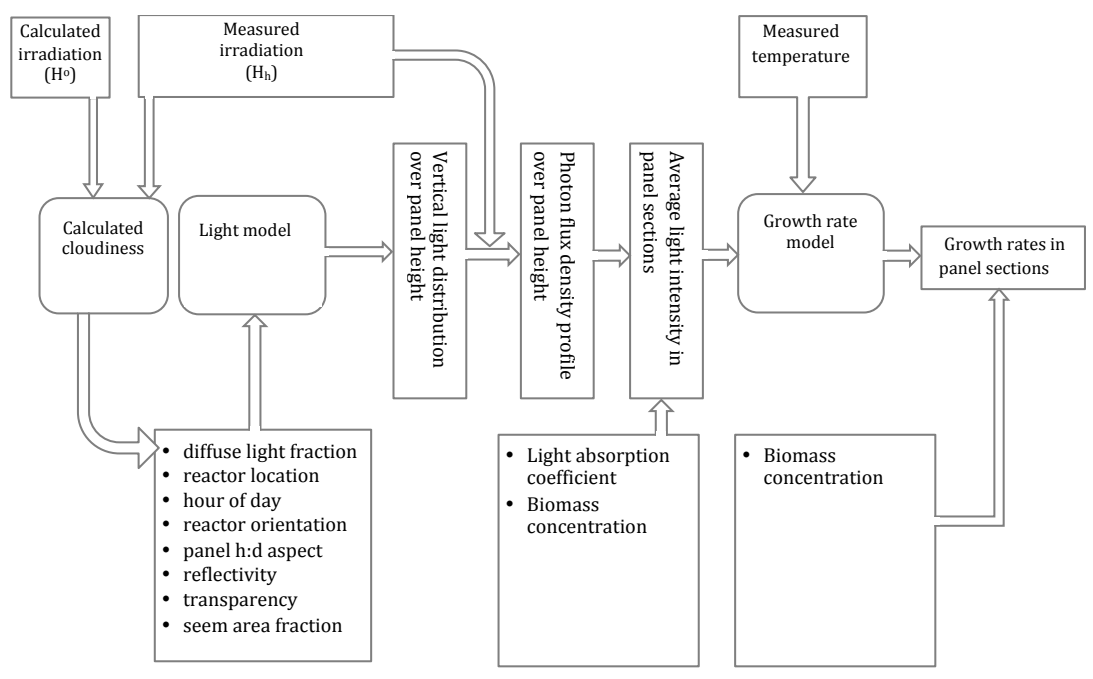

Fig. 4-4 Model flow diagram. The model simulates specific growth rate from only two free variables: reactor temperature and horizontal irradiation.

1. Light model: The vertical profile of panel surface PFD (photon flux density) was calculated from horizontal irradiation $\left(\mathrm{H}_{h}\right)$ using a ray tracing model. The model needs as an input the proportion of diffuse light which was estimated from the cloudiness, using the discontinuous equation 6 of Collares-Pereira (Collares-Pereira et al. 1979). Cloudiness is given as the proportion of measured horizontal irradiation to horizontal irradiation without the influence of atmosphere $\mathrm{H}^{\circ}$. Equations for $\mathrm{H}_{h}$ and $\mathrm{H}^{\circ}$ were found in Duffie and Beckmann (Duffie et al. 1991).

2. Calculation of panel light intensity: Light intensity in the panel was calculated as average light intensity from panel surface PFD, using the integrated Lambert-Beer formula.

3. Growth rate model: Calculates the current growth rate of Nannochloropsis in each of 20 vertically stacked segments of a unit panel as a function of panel segment average light intensity and reactor temperature.

4. Calculation of productivity rate. The growth rate is then multiplied with biomass concentration and summed over the panel to obtain instantaneous panel productivity. The biomass concentration used for this purpose was a daily measured value, common for all reactors (as sampled from the harvested, mixed culture). 
Daily productivity was calculated from the productivity rate, summing over the daytime hours. At night, the model is not applicable.

\section{Light model}

The ray-tracing model Raypro was used to calculate the vertical profile of light falling on the vertical surface of a unit reactor panel, meaning that there was no distinction made between reactor panels which is a simplification as the first panel in the reactor is obviously differently lit from the others. At low solar elevation, the first panel in a reactor, oriented with its longitudinal axis towards the sun would receive a much larger irradiation that the others and could bias the result. But as one panel represents less than $3 \%$ of the total panel surface or volume of the reactor, a deviation of the light absorption of the first panel from the others would weigh on the average of the others by only $3 \%$. The reactors in the set-up were oriented north-south so it was decided not to try to shade the first panel. The panel was considered uniformly irradiated over the width. Irradiation, normally reflected between panels was included to the unit panel at the appropriate vertical position. Light was not considered to pass through the panels. This fully acceptable at normal operating biomass densities because almost all light will be absorbed during the passage of the panel: with a biomass density of $3 \mathrm{~g} D W$ per liter, direct light is more than 99\% absorbed at the end of the path through the panel, using Lambert-Beers formula. With a biomass density of $0.05 \mathrm{~g} \mathrm{DW} \mathrm{L}^{-1}$, on the other hand, only $10 \%$ of the direct light would be absorbed at the end of the path and consequently, the model would underestimate productivity, but such low biomass densities have little practical relevance under outdoor conditions. Welding seams in the panels (creating the vertical tubes) were considered not to absorb any light.

Model input parameters were the following:

1. horizontal irradiation, measured with flat LiCor L-190 quantum (PAR) sensor

2. reactor aspect (ratio between panel distance and panel height): 0.3

3. fraction diffuse light

4. geographical position (latitude, longitude)

5. reactor orientation

6. time of year 
7. reflectiveness from film cover: $10 \%$ (estimate)

8. bottom reflection layer: $90 \%$ (producer specification, verified empirically)

9. Percentage welding seam of panel area: $10 \%$

The proportion of direct to diffuse light, required for the ray-tracing mode was estimated from the atmospheric clearness index, $\mathrm{K}_{\mathrm{h}}$, according to the discontinuous equations in Collares-Pereira (Collares-Pereira et al. 1979):

$K_{h}=\frac{H_{h}}{H_{0}}$ :diffuse light proportion (Equation 4-3)

$\left(H_{h}\right)$ is the measured irradiance on the horizontal surface, $H_{0}$ the calculated irradiance for the surface without atmosphere.

The algae reactor's geometry was translated into a collection of surfaces in 3D with individual optical properties such as refractive index, diffuse reflectance and light absorption coefficient as a function of the wavelength. The reactor panel was divided into several vertical sections in order to calculate the vertical light distribution over the reactor panel.

Average light intensity in panel compartment $I_{a v}\left(\mu \mathrm{mol} \mathrm{sec}{ }^{-1} \mathrm{~m}^{-2}\right)$ was calculated with the integrated Lambert-Beer formula:

$I_{a v}\left[\mu \operatorname{molsec}^{-1} m^{-2}\right] \quad=\frac{I_{0}}{p * C_{b} * K_{a}}\left(1-\exp \left(-p * C_{b} * K_{a}\right)\right)$

(Equation 4-4)

with:

$I_{0}\left[\mu \mathrm{mol} \mathrm{sec}{ }^{-1} \mathrm{~m}^{-2}\right] \quad$ :photon flux density at surface of panel section

$p[m]$

: light path

$C_{b}\left[k g ~ m^{-3}\right]$

: biomass concentration

$K_{a}\left[m^{2} k^{-1}\right]$

:light absorption coefficient

Spatial light absorption coefficient, $\mathrm{K}_{\mathrm{a}}=189 \mathrm{~m}^{2} \mathrm{~kg}^{-1}$ was measured with a low-light adapted, nutrient replete batch culture of Nannochloropsis oceanica, using a spectrophotometer with an integrating sphere:

$K_{a}\left[m^{2} * k^{-1}\right]=\bar{A}_{400-700 n m} * F * \ln (10) * L^{-1} * D W^{-1}$ (Equation 4-5) with:

$\bar{A}_{400-700 n m}$ : average absorbance, $400 \mathrm{~nm}$ to $700 \mathrm{~nm}$, 
(1 $\mathrm{nm}$ interval).

F

$L[m]$

$D W\left[\mathrm{~kg} \mathrm{~m}^{-3}\right]$
: dilution factor for cuvette

:cuvette light path $(0.06 \mathrm{~m})$

:cuvette biomass concentration

\section{Construction of the growth rate model}

To keep the number of parameters as low as possible, two different models were combined: With respect to light intensity, the binomial growth rate expression of Geider was used (Geider et al. 1997) and to incorporate the temperature effect on the growth rate, the expression of Blanchard (Blanchard et al. 1996) was used. Taking two parameters as one product and fixing another parameter at its initially simulated value, the number of parameters was $5\left(T_{\text {let }}, T_{\text {opt }}, \beta, \mu_{\text {max }}, \alpha * \theta\right)$.

For the effect of light intensity, I on the growthrate $(\mu)$ Geiders equation 7 (Geider et al. 1997) was used:

$\mu=P_{m}^{c} *\left(1-\exp \left(\frac{-\alpha * \theta * I}{P_{m}^{c}}\right)\right)-r_{m} \quad[\sec -1]$ (Equation 4-6)

$P_{m}^{c}$, the light saturated, carbon specific photosynthesis rate, relates to temperature through:

$P_{m}^{c}=\mu_{\max } * f_{T}+r_{m} \quad\left[\sec ^{1}\right]$ (Equation 4-7)

$\theta$ is the chlorophyll a:cell carbon ratio; $\propto$ is the initial slope of the $\mathrm{PI}$ curve $\left[\mathrm{m}^{2} \mathrm{gC} \mathrm{mol}^{-1}\right.$ photons $\left.\mathrm{g}^{-1} \mathrm{Chla}\right] ; r_{m}$ is the metabolic maintenance $\left[\mathrm{sec}^{-1}\right]$. $\mu_{\max }$ is the maximum specific growth rate and $f_{T}$ is a variable that describes the temperature effect on the growth rate. $f_{T}$, was taken according to Blanchard et al (Blanchard et al. 1996):

$f_{T}=\left(\frac{T_{\text {let }}-T_{w}}{T_{\text {let }}-T_{\text {opt }}}\right)^{\beta} * \exp \left(-\beta *\left(\frac{T_{\text {let }}-T_{w}}{T_{\text {let }}-T_{\text {opt }}}-1\right)\right)$ (Equation 4-8) $T_{w}$ is the short time variable temperature, $T_{\text {opt }}$ is the temperature optimum for $T_{\text {let }}$ is the maximum sustainable temperature while $\beta$ is a dimensionless shape factor.

In the current work, the parameters were determined by non-linear parameterfitting of the Equations 6-8 to 4 temperature sets of dilution rate measurements with varying irradiation. $\theta$ and $\propto$ were determined as one single parameter $(\theta * \propto)$ and $r_{m}$ was first 
included in the parameter fitting as a variable but then fixed at its fitted value, 0.05 to reduce the confidence intervals.

The fit was done in MATLAB R2015b by the function nlinfit. The coefficients were estimated using iterative least squares estimation. The $95 \%$ confidence intervals were derived with the nlparci function.

\section{Simulated productivity calculation}

$P R_{\text {sim }}$, the simulated productivity rate of the reactor, is the sum of biomass productivity, calculated for for each of the 20 vertical panel sections:

$P R_{\text {sim }}\left[g^{-2} h r^{-1}\right]=\sum_{1}^{20} \frac{\mu * C_{b} * L}{a s p * 24}$ simulated productivity rate (Equation 4-9) with:

$\mu\left[\right.$ day $\left.^{-1}\right] \quad$ :instantaneous growth rate in a panel compartment, as predicted by growth model as function of average light intensity in panel section and temperature.

$C_{b}\left[\mathrm{~kg} \mathrm{~m}^{-3}\right] \quad$ :biomass concentration in photobioreactor

$L[m] \quad$ :avg. panel depth

asp : :distance between panels, divided by panel height

Simulated daytime productivity was calculated as:

$P_{\text {dsim }}\left[g D W m^{-2}\right]: \sum_{\text {sunrise }}^{\text {sunset }} P R_{\text {sim }} \Delta \mathrm{t}$ simulated daytime productivity (Equation 4-10)

\section{Empirical productivity calculation}

To measure productivity, $\mathrm{CO}_{2}$-consumption/uptake in the industrial photobioreactor was used to estimate area productivity rate with high resolution rather than direct biomass measurements. The reason for using $\mathrm{CO}_{2}$-consumption/uptake to calculate productivity rather than direct biomass measurement, is that the latter depend on either laborious sampling procedures or on complex in-line turbidity or electrochemical biomass sensors. Measurement of carbon dioxide in the recirculating gas is simple, fast and reflect overall culture biomass productivity. During the operation of the plant, the recirculating gas was 
diluted with a flow of atmospheric air to establish gas exchange with the ambient and a controlled concentration of $\mathrm{CO}_{2}$, normally $1.5 \%$ was maintained in the recirculating gas. The $\mathrm{CO}_{2}$-consumption/uptake was calculated from the $\mathrm{CO}_{2}$ mass flow into the plant, minus the mass flow of $\mathrm{CO}_{2}$, leaving the plant. It is difficult to indicate the accuracy of the the $\mathrm{CO}_{2}$ uptake calculation accuracy. Carbon mass flow into the plant (all 20 reactors) was controlled with a mass flow $\mathrm{CO}_{2}$ controller (about $3 \%$ accuracy). Carbon mass flow out of the reactor was calculated from $\mathrm{CO}_{2}$ concentration, measured with a $\mathrm{CO}_{2}$ sensor in the recirculating gas and the gas exchange with the ambient, as controlled with an on-off operated diaphragm pump. The $\mathrm{CO}_{2}$ sensor was a Vaisala model GMT221 in combination with Vaisala GMT 220 transmitter with an accuracy of $1.5 \%$ of range $\left(10 \% \mathrm{CO}_{2}\right)$ and a precision of $2 \%$ of the reading. The accuracy of the operation of the diaphragm pump was not precisely known but believed to be high, probably in the order of 1-2 \%. However, regulatory events caused deviations, for example following planned and unintentional deflations of the plant. The dynamics of the regulation has not been fully documented but considered to be sufficiently responsive: Solar events were tracked with about 10 minutes delay. Carbon dioxide loss through leaks in the system (either punctures or diffusion through the plastic film) would wrongly be registered as productivity but as the system could be maintained with pure medium for several days without any productivity being registered (data not shown), it appears to be $\mathrm{CO}_{2}$ tight. A matter of concern with using $\mathrm{CO}_{2}$-consumption/uptake to estimate productivity is that a fraction of the $\mathrm{CO}_{2}$ absorbed by the medium might accumulate in the medium as hydrogen carbonate and carbonate and incorrectly be registered as productivity. However, the effect is small. For example, in a semicontinuous culture, operated at similar $\mathrm{pH}$ and dilution rate, dissolved carbon in the medium was $50 \mathrm{mg} \mathrm{C/L}$ or $4.2 \mathrm{mM}$ (Acién Fernández et al. 2003). On a cloudless summer day, this would result in a day-time, productivity overestimation of $2.3 \mathrm{~g} \mathrm{DW} \mathrm{m}^{-2}=6 \%$. With the 24 hour productivity figure, carbonate formation would be of even less significance as carbonates are assumed to partly dissolve during night. Leaks occurring as accidents, on the other hand, did take place but could be verified as increased air demand to keep the system pressurized. 
Constants:

$E\left[k J g^{-1}\right]$

:enthalpy of biomass:

19.2

$\mathrm{CDW}$

:biomass-carbon fraction:

$R\left[\mathrm{~mol}\left[L *{ }^{\circ} \mathrm{K}\right]^{-1}\right]$

:gas constant:

0.08206

$A R\left[m^{2}\right]$

:Total, panel covered reactor area:

140

Measured parameters:

$P A R\left[\mu \mathrm{mol} \mathrm{m}^{-2} \mathrm{sec}^{-1}\right] \quad$ : horizontal incident irradiation (PAR)

$\mathrm{F}_{\mathrm{CO}_{2}}\left[\mathrm{~mol} \mathrm{~m}^{-2} \mathrm{hr}^{-1}\right] \quad$ : Flow $_{\text {in }}$ of $\mathrm{CO}_{2}$ at standard conditions

$\mathrm{X}_{\mathrm{CO}_{2}}$

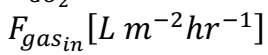
:mass fraction $\mathrm{CO}_{2}$ in circulating gas.

$Q_{\mathrm{CO}_{2}}\left[\mathrm{~mol} \mathrm{~m}^{-2} \mathrm{hr}^{-1}\right]$

:Flow of gas into reactor

:Flow of $\mathrm{CO}_{2}$ into reactor

Equations

$\begin{array}{ll}Q_{\mathrm{CO}_{2}}\left[\mathrm{~mol} \mathrm{hr} r^{-1} \mathrm{~m}^{-2}\right] & =\left(F_{\mathrm{CO}_{2}}-X_{\mathrm{CO}_{2}}\right. \\ & : \text { Flowrate of } \\ P R_{\text {empir }}\left[\mathrm{g} \mathrm{m}^{-2} \mathrm{hr}^{-1}\right] & =\frac{12 Q_{\mathrm{CO}_{2}}}{\mathrm{CDW}} \\ & : \text { Empirical prod }\end{array}$

(Equation 4-11)

:Flowrate of $\mathrm{CO}_{2}$ into reactor

:Empirical productivity rate

$P_{\text {dempir }}\left[g^{-2} h r^{-1}\right]$

$=\sum_{\text {sunrise }}^{\text {sunset }} P R_{\text {empir }} \Delta t$

(Equation 4-13)

:Daytime empirical productivity

$P_{24 \text { empir }}\left[\mathrm{g} \mathrm{m}^{-2} \mathrm{hr}^{-1}\right] \quad=\sum_{0}^{24} P R_{\text {empir }} \Delta t$

(Equation 4-12)

:24-hour empirical productivity

$I R\left[M J m^{-2}\right.$ day $\left.^{-1}\right] \quad=\sum_{\text {sunrise }}^{\text {sunset }} \frac{P A R}{0.43 * 4.6}$

(Equation 4-15)

:daily horizontal solar irradiation

PE [\%]

$$
=P d_{\mathrm{CO}_{2}} * \frac{E}{I R} * 100
$$

:photosynthetic efficiency

For photosynthetic efficiency calculations and calculation of proportion of indirect light, PAR quanta ( $\mu \mathrm{M} \mathrm{m}^{-2} \mathrm{sec}^{-1}$ ) were converted to energy total solar radiation to total solar irradiation $\left(\mathrm{W} \mathrm{m}^{-2}\right)$ by the factors $\frac{1}{4.6}$ and $\frac{1}{0.43}$ (Slegers et al. 2011). 
For the photosynthetic efficiency calculation, a mass-enthalpy value of $19.2 \mathrm{~kJ} \mathrm{~g} \mathrm{dw}^{-1}$ was applied (both to modeled and measured productivity) and for the calculation of observed biomass productivity in the photobioreactor, a carbon mass fraction of biomass of 0.42 for nutrient replete Nannochloropsis was used - which represents the only "judicious" coefficient used in the validation (the term used with reference to Huesemann et al. (Huesemann et al. 2016). Biomass-based enthalpy and C: biomass ratio was calculated, using average enthalpy $\mathrm{C}-\mathrm{mol}^{-1}$ and elemental analysis of $\mathrm{C}, \mathrm{H}, \mathrm{N}$ and $\mathrm{O}$ for Chlamydomonas, 2 Chlorella strains, one Selenastrum and one Scenedesmus strain (Duboc et al. 1999) with biomass $\mathrm{C}-\mathrm{mol}^{-1}$ for elements other than $\mathrm{C}, \mathrm{H}, \mathrm{O}$ and $\mathrm{N}$ taken from average values of 20 microalgal strains (Ho et al. 2003). Calculated enthalpy of biomass was $19.20 \mathrm{~kJ} \mathrm{gdw}^{-1}$. The values agree with other literature values: while $\mathrm{C}: \mathrm{CHON}$ ratio for nutrient replete Nannochloropsis sp. and Nannochloropsis salina were 60.97 and $59.5 \%$ respective (Raso et al. 2012, Sforza et al. 2012); C:DW was 43.3\% for Nannochloropsis sp. in paste from Reed Mariculture (Brown et al. 2010). With 2 different $\mathrm{CO}_{2}$ concentrations in the spargeing gas, 42.8, respectively 44.8 C \% CNHO was obtained for N. oculata (Hsueh et al. 2009).

\section{Test of model with ProviApt reactor plant.}

1. To test how the model predicted outdoor productivity, 3 days with irradiation extremes were modeled and compared with $\mathrm{CO}_{2}$-absorption based productivity data for the Proviron production site in Antwerp:

2. a cloudless summer day (09.09.2012): an example of a rather pure sinusoid radiation pattern that will allow simple visual comparison of the modeled and measured productivity. The irradiation on the day was $21.08 \mathrm{MJ} \mathrm{m}^{-2}$

3. a variable-cloud cover day (12.09.2012): a day, close to the first so that the extraterrestrial irradiation is similar but with strongly variable cloud cover to allow comparison of model performance under continuous and variable irradiation conditions. The irradiation on the day was $16.40 \mathrm{MJ} \mathrm{m}^{-2}$.

4. a variable-cloud cover winter day (03.01.2013): a day with minimum irradiation to test compliance at minimum irradiation which in terms of solar elevation and direct/in-direct proportion is another extreme situation. 


\section{Results and discussion}

\section{Algal steady states}

20 steady state turbidostat runs with Nannochloropsis oceanica were obtained in the laboratory turbidostat. Dilution- and biomass data from these are presented in Table 4-2.

\begin{tabular}{|c|c|c|c|c|c|c|c|c|c|}
\hline $\begin{array}{l}\text { Steady } \\
\text { state \# }\end{array}$ & $\begin{array}{l}\text { Light } \\
\text { intensity } \\
{\left[\mu \mathrm{mol} \mathrm{m}^{-2}\right.} \\
\left.\mathrm{sec}^{-1}\right]\end{array}$ & $\begin{array}{l}\text { Temp } \\
{\left[{ }^{\circ} \mathrm{C}\right]}\end{array}$ & $\begin{array}{l}\text { Dilution } \\
\text { rate } \\
{\left[\text { day }^{-1}\right]}\end{array}$ & $\begin{array}{l}\text { Stddev, } \\
\text { dilution } \\
\text { rate }\end{array}$ & $\begin{array}{l}\text { Slope, } \\
\text { dilution } \\
\text { rate. } \\
{\left[\text { day-2 }^{-2} \text {. }\right.}\end{array}$ & $\begin{array}{l}\text { Biomass } \\
\text { conc. } \\
\text { [g DW L-1] }\end{array}$ & $\begin{array}{l}\text { Stddev, } \\
\text { biomass } \\
\text { conc. }\end{array}$ & $\begin{array}{l}\text { Slope, } \\
\text { biomass } \\
\text { [g DW L L } \\
\text { day }^{-1} \text { ] }\end{array}$ & $\begin{array}{l}\text { TFA } \\
{[\% \text { DW] }}\end{array}$ \\
\hline 1 & 100 & 20 & 0.78 & 0.146 & 0.002 & 0.16 & 0.047 & 0.0148 & \\
\hline 2 & 100 & 25 & 0.74 & 0.112 & 0.000 & 0.08 & 0.020 & 0.008 & \\
\hline 3 & 100 & 30 & 0.68 & 0.029 & -0.012 & 0.10 & 0.016 & 0.002 & 5.2 \\
\hline 4 & 33 & 20 & 0.35 & 0.033 & 0.000 & 0.11 & 0.009 & 0.007 & \\
\hline 5 & 33 & 15 & 0.23 & 0.045 & -0.001 & 0.15 & 0.019 & -0.001 & 3.3 \\
\hline 6 & 37 & 25 & 0.37 & 0.043 & 0.036 & 0.09 & 0.051 & 0.003 & 3.6 \\
\hline 7 & 37 & 30 & 0.15 & 0.042 & -0.009 & 0.09 & 0.007 & -0.002 & 6.2 \\
\hline 8 & 160 & 25 & 0.60 & 0.109 & -0.018 & 0.13 & 0.013 & -0.001 & 3.5 \\
\hline 9 & 187 & 30 & 0.71 & 0.144 & 0.012 & 0.10 & 0.022 & -0.012 & 6.7 \\
\hline 10 & 187 & 25 & 0.80 & 0.133 & 0.030 & 0.08 & 0.015 & -0.001 & 6.3 \\
\hline 11 & 205 & 25 & 0.70 & 0.054 & 0.000 & 0.13 & 0.025 & 0.000 & 6.3 \\
\hline 12 & 205 & 30 & 0.75 & 0.098 & -0.067 & 0.14 & 0.020 & -0.018 & 3.9 \\
\hline 13 & 137 & 25 & 0.68 & 0.066 & -0.017 & 0.16 & 0.009 & 0.005 & 7.0 \\
\hline 14 & 173 & 20 & 0.64 & 0.110 & 0.014 & 0.16 & 0.014 & -0.004 & \\
\hline 15 & 200 & 20 & 0.74 & 0.108 & 0.000 & 0.14 & 0.015 & 0.001 & 6.8 \\
\hline 16 & 29 & 15 & 0.26 & 0.053 & 0.002 & 0.10 & 0.017 & 0.000 & 7.7 \\
\hline 17 & 27 & 20 & 0.42 & 0.167 & 0.010 & & & & \\
\hline 18 & 27 & 25 & 0.47 & 0.078 & 0.006 & 0.07 & 0.006 & 0.000 & 4.1 \\
\hline 19 & 25 & 30 & 0.40 & 0.099 & 0.000 & 0.07 & 0.013 & -0.004 & 3.3 \\
\hline 20 & 150 & 25 & 0.89 & 0.053 & 0.011 & 0.18 & & & \\
\hline
\end{tabular}

Total fatty acids as percentage dry weight ranged from 3.3 to 7.7 TFA\% DW with no trend with respect to irradiation level (data not shown). Under nutrient limitation, fatty acid contents may be much higher: with various levels of nutrient limitation during batch growth, total fatty acid contents of Nannochloropsis sp. CCAP 211/78 varied between 9.1 and 28.1\% DW (Hulatt et al. 2017) and 22-37 \% DW (Solovchenko et al. 2014). 
The low fatty acid contents in the turbidostat samples in the current study support the notion that the growth rates obtained were the maximum possible under the light and temperature conditions.

The turbidostat maintained an $\mathrm{OD}_{680}$ value of 0.4 which resulted in steady state biomass in the reactor, ranging from 68 to $180 \mathrm{mg} \mathrm{DW} \mathrm{L}^{-1}$ (Table 4-2). At this biomass level, the light transmission through the culture was around $80 \%$ of that of a reactor filled with water (data not shown). This means that the real light intensity, experienced by the algae at any time has been in a narrow range between the stated light intensity and a value, $25 \%$ higher.

The coefficients in equations 6-11 were fitted to the dilution rates values from Table 4-2, excluding 4 values (steady state number 8,14,17 and 19) as these values deviated strongly from the trend of the irradition series and the biomass concentration of the cultures indicated that a steady state had not been safely established.

It was not possible to find quality criteria for algal turbidostat steady states in the literature which could be helpful to determine when a new steady state has been reached. This is probably particularly important with turbidostat cultures where the optical property of the culture (that triggers the dilution) depend on many parameters.

Slow or apparently suspended acclimation to a new condition may possibly explain deviating points as for example with phosphate limited Pavlova cultures (Droop 1974) or light limited Dunaliella cultures (Sciandra et al. 1997).

Fluorescent steady-state quantum yield versus irradiation of the turbidostat cultures is depicted in Fig. 4-5.

The quantum yield of the algae in the different steady states appear to depend only on light intensity. At low light intensity, $\left(25 \mu \mathrm{mol} \mathrm{m}^{-2} \mathrm{sec}^{-1}\right)$, the quantum yield was $>0.7$ whereas at above $200 \mu \mathrm{mol} \mathrm{m} \mathrm{mec}^{-1}$, the quantum yield was $<0.2$. The regression line in Fig. 4-5 intersects the abscissa at $233 \mu \mathrm{mol} \mathrm{m}^{-2} \mathrm{sec}^{-1}$, i.e. thus represents the zero-QY irradiation level which must be assumed to be the maximum continuous light intensity, that Nannochloropsis oceanica can sustain.

Other works report growth of Nannochloropsis sp. or N.salina under continuous irradiation at higher intensities (250-500 $\mathrm{mmol} \mathrm{m}^{-2} \mathrm{sec}^{-1}$ ) (Sukenik 1991, Van Wagenen et al. 2012). Spolaore on the other hand, found an optimum light intensity of $52 \mu \mathrm{mol} \mathrm{m}^{-2} \mathrm{sec}^{-1}$ at $21 \mathrm{deg}$ 
(Spolaore et al. 2006) with N. oculata.

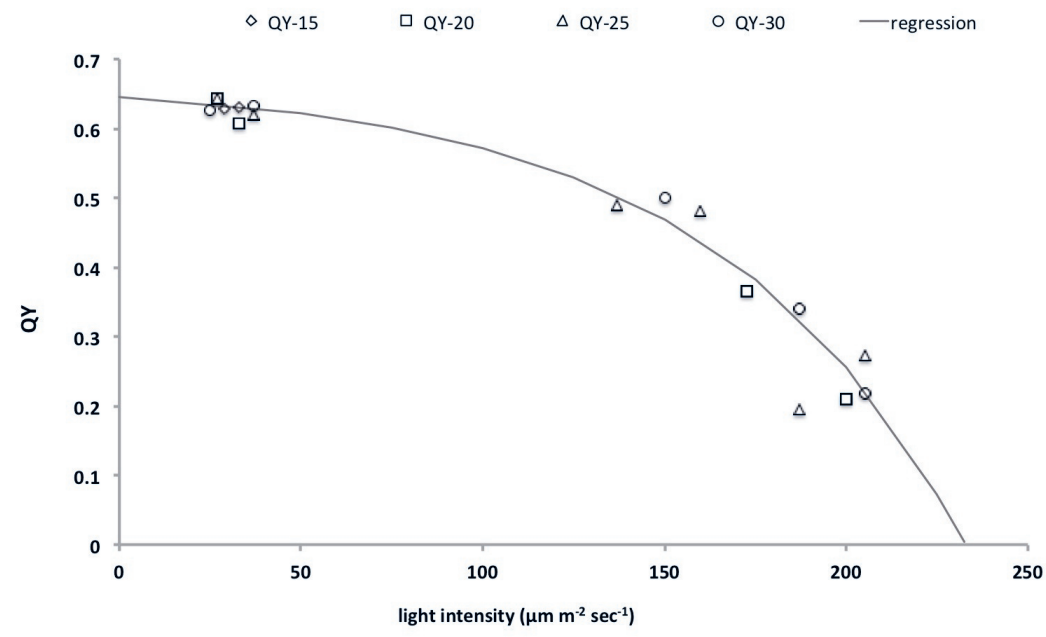

Fig. 4-5 Quantum yield (QY) of Nannochloropsis oceanica in steady state turbidostat cultures. Symbols: QY-15 stands for quantum yield of the 15 degrees series and so forth. The regression line is fitted to the average $Q Y$ at the various light intensity levels through the expression $Q Y=a *(b-\exp (c * x))$ using least squares method and the Excel tool "Solver". The QY curve intersects the abscissa at a light intensity of $233 \mathrm{\mu mol} \mathrm{m}^{-2} \mathrm{sec}^{-1}$.

This apparent inconsistency could depend on differences in light measurement, optical path or applied biomass concentration. 


\section{Parameter fitting.}

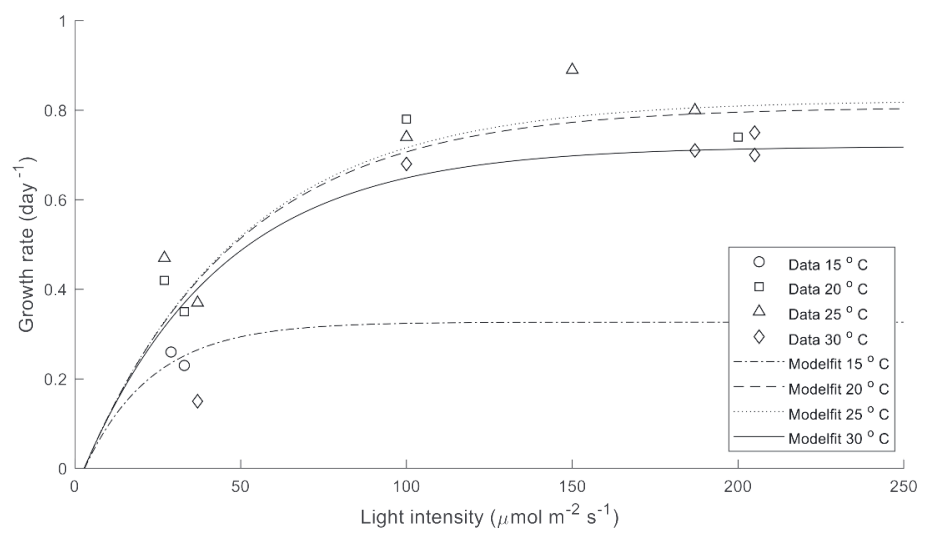

Fig. 4-6 4 temperature sets of growth rate versus light intensity and fitted curves. Note that the 15 degrees only holds two measurements at low light as attempts operating at higher irradiation failed. This means that the applicability of the curve at higher irradiation failed. This means that the applicability of the curve at higher irradiation can not be known.

Fig. 4-6 shows the growth rates obtained 4 light intensity series performed at different temperature on the laboratory turbidostat reactor and the curves fitted to the data.

Table 4-3 Fitted values for constants in growth rate equation and their confidence intervals (95\%).

\begin{tabular}{|l|l|l|l|l|}
\hline & $\begin{array}{l}\text { Fixed } \\
\text { value }\end{array}$ & $\begin{array}{l}\text { Parameter } \\
\text { fit result }\end{array}$ & $\mathrm{Cl}-$ & $\mathrm{Cl}+$ \\
\hline Tlet & 14.265 & 7.2735 & 21.258 \\
\hline Topt & 22.633 & 16.301 & 28.965 \\
\hline$\beta$ & 0.6206 & -2.256 & 3.497 \\
\hline$\mu_{\max }$ & 0.8396 & 0.6763 & 1.0028 \\
\hline$r_{\mathrm{m}}$ & 0.05 & & & \\
\hline$\alpha * \theta$ & 0.2127 & 0.1344 & 0.2910 \\
\hline
\end{tabular}

The fitted values of the 6 growth equation constants and their confidence intervals are given in Table 4-3. After the initial fit of all 6 constants that resulted in large confidence intervals, $r_{m}$ was fixed at its fitted value $(0.05)$ and the calculation repeated which resulted in fitted values, shown in Table 4-3.

Normalized mean absolute error was $11.7 \%$.

The reliability of the fit was further evaluated by using a reduced training set (n-1) and testing the results on the excluded data ( the leave-one-out procedure, see appendix). Training the model to the $n-1$ dataset increased the deviation of the fit. The normalised mean absolute errors of the fit (relative root mean square errors) ranged between $0.4 \%$ and $202.4 \%$ and averaged $21.5 \%$. The difference from using the full dataset is mainly due to 3 values dataset which could indicate overfitting. However the full dataset is used rather than using 
the cross validation to tune the coefficients in accordance with the guidelines in (Hawkins 2004).

\subsection{Modeled growth rate}

The surface plot in Fig. 4-7 was made by entering the fitted coefficients from Table 4-3 in the growth rate model, Equations 6 to 8 .

The experimental area of validity of the equation for continuous illumination extends only to an irradiation of $205 \mu \mathrm{m} \mathrm{m}^{-2} \mathrm{sec}^{-1}$ which is the maximum light intensity that was at which continuously illuminated cultures were established. Fig. 5 indicates that the light adapted fluorescent quantum yield has reached 0 at irradiation $>233 \mu \mathrm{m} \mathrm{m}^{-2} \mathrm{sec}^{-1}$ which suggests that the capacity for non-photochemical quenching of the algae has been reached, including xanthophyll cycle-, state transition- and photoinhibition reactions (Müller et al. 2001). Sustained exposure to irradiation above that level may result in photoinhibition. To judge the extent of such high light intensity exposure, it is usefull to examine the timespatial distribution of average light intensity $\left(\mathrm{l}_{\mathrm{av}}\right)$ in the industrial photobioreactor. In Fig. $4-8$, the occurrence of average light intensity above $250 \mu \mathrm{m} \mathrm{m}^{-2} \mathrm{sec}^{-1}$ on a cloudless summer day is indicated in yellow-red signatures. This occured from 18 to $19 \mathrm{hrs}$ and sporadically also at the bottom segments during noon, which must be judged acceptable.

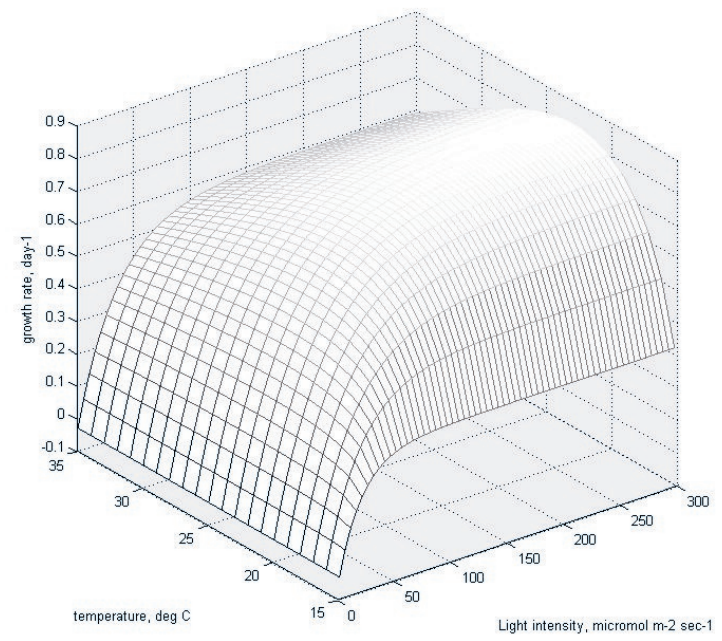

Fig. 4-7 Nannochloropsis growth rate as function of light intensity and temperature. 


\section{Limitations of the overall modeling approach}

Measuring light and temperature limited growth rates as steady-state values has some limitations; first, the algal culture under consideration must be nutrient replete. Fatty acid enhancement by nutrient depletion can therefore not be applied. Secondly, the growth rate model is produced with dilute, continuously illuminated steady state cultures and a maximum sustainable light intensity can be derived from these steady states. Above or around the maximum continuously sustainable light intensity, the cultures become unstable and may aggreagate or adhere to the walls of the reactor. It would be difficult, therefore to obtain stable measurements at irradiation levels above the $205 \mu \mathrm{mol} \mathrm{m} \mathrm{mec}^{-1}$ which was the maximum tested. In the industrial photobioreactor, the light intensity experienced by the algal culture may for shorter periods of time exceed the continuously sustainable maximum. In practice this could happen as a consequence of a low biomass concentration or low absorption coefficient and would result in the irradiation being outside the test field. Mapping the calculated light intensity in the culture is therefore necessary to justify the validity of the growth rate model. This is shown in Fig. 4-8. for the cloudless summer day, where the zones (space-time) with irradiation above the test field are graphically easily observed. The computational consequences of exeding the tested irradiation is that the calculated growthrate would remain at the maximum level as there is no photoinhibition mechanism built in. Raising the biomass concentration in the reactor would remedy the situation. Orientation of the reactor wil also influence light exposure.

\subsection{Light absorption of the industrial photobioreactor}

The light absorption model predicts absorption between $50-80 \%$ of the incident irradiation for most of the hours of the day (Fig. 4-10). For a given site, this factor is a clear object for optimization of the performance of the plant; factors influencing the light absorption of the reactor include biomass concentration, absorption coefficient, orientation of the reactor, panel aspect ratio, reflectance of the material, reflectance and transmissivity of the reactor film. Orientation of the reactor, for example influence absorption efficiency of the reactor by $10 \%$ (data not shown). 


\section{Simulated and empirical productivity asessments}

\section{Cloudless summer day}

Fig. 4-9 shows the resulting growth rates and in Fig. 4-11, the predicted productivity on the cloudless summer day, showing both simulated and empirical productivity. Night-time respiratory biomass loss was $23 \%$ of the biomass. It is interesting that the rather short duration spikes in irradiation occurring in the morning are reflected both in the simulated and empirical productivity and suggests that the $\mathrm{CO}_{2}$ - consumption/uptake is a responsive indicator of productivity.

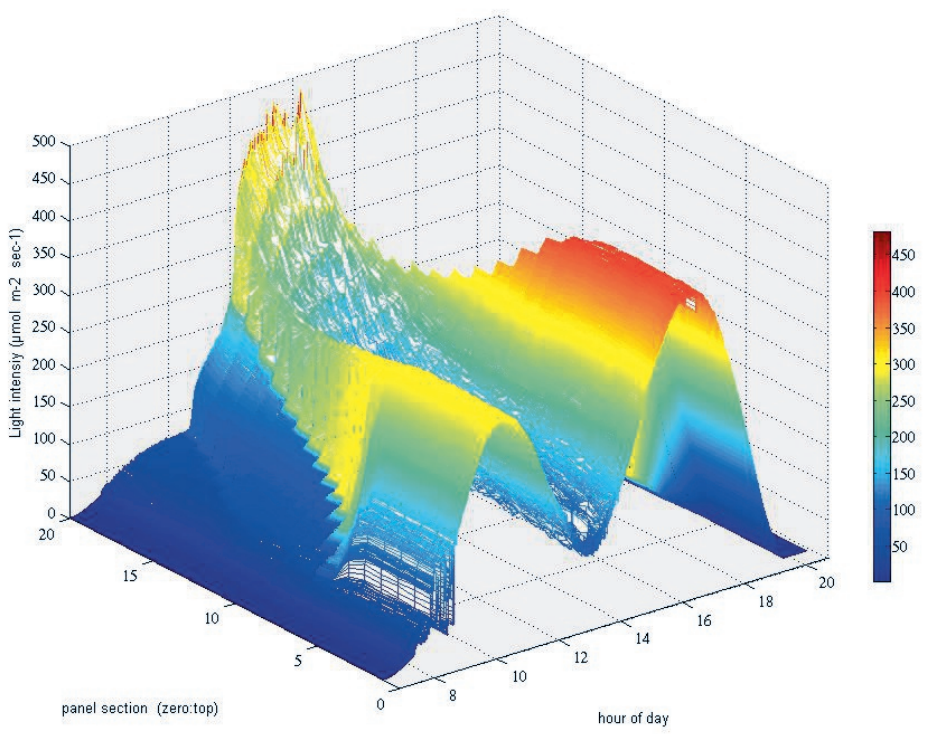

Fig. 4-8 Average light intensity in panel sections on a cloudless summer day (09.09.2012).

Blue-green colors mark a average light intensity level under $250 \mu \mathrm{mol} \mathrm{m}^{-2} \mathrm{sec}^{-1}$. 


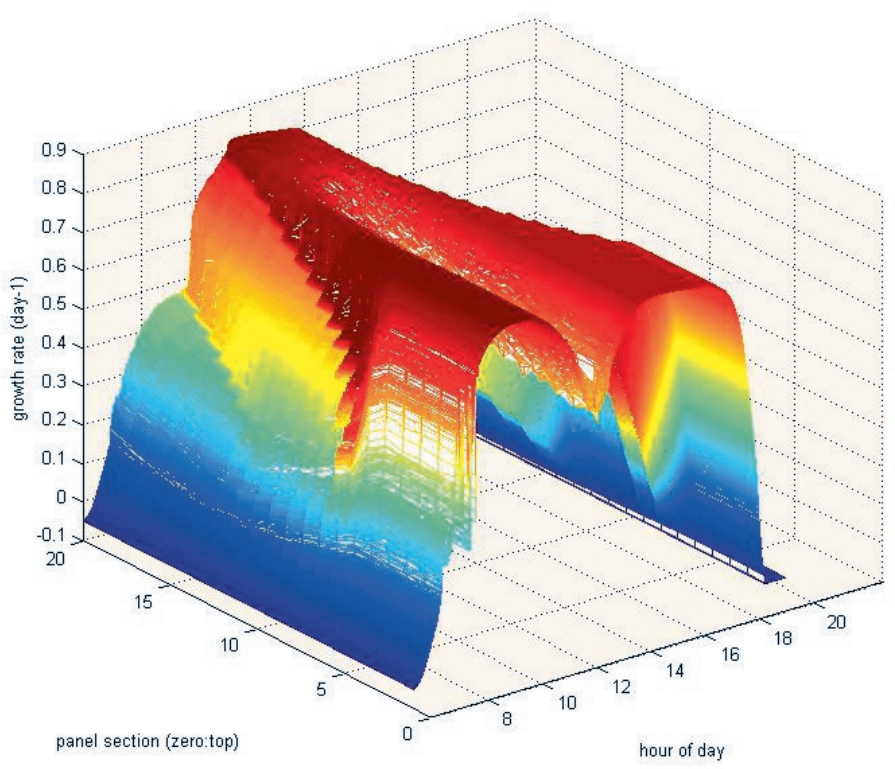

Fig. 4-9 Simulated algal growth rate in photobioreactor compartments on a cloudless summer day (09.09.2012)

There is generally a good correlation except for the midday hours where the simulated productivity rate is systematically lower than the empirical. It may be due to reflected irradiation from the bottom film, covering the bottom of the reactors between the panelcovered areas, which is not handled by the model. The simulated daytime productivity is 15 \% lower than the empirical (Table 4-4).

\section{Cloudy summer day}

For a summer day with strongly variable cloud cover (Fig. 4-12), the simulated productivity exhibits such a dynamic response to the irradiation that it was difficult to compare with the empirical productivity. In , the simulated productivity curve therefore was smoothed by rolling average of 20 points (that is, over a period of 100 seconds). The general trend of the simulated and empirical productivity graph appears rather coinciding.

Simulated daily productivity was $8.1 \%$ lower than the empirical (Table 4-4). 
Night time respiratory biomass-loss was $20 \%$. The empirical data included some unusual peaks that could have been caused by mechanical malfunction or transients in the $\mathrm{CO}_{2}-$ control of the plant.

\section{Cloudy winter day}

On the cloudy winter day with irradiation $0.89 \mathrm{MJ}^{\text {day }}{ }^{-1}$ (Fig. 4-13), simulated and empirical daytime productivity was around $2 \mathrm{~g} \mathrm{dw} \mathrm{dw} \mathrm{m}^{-2}$ day $^{-1}$ (Table 4-4).

Due to night time respiration, the 24 -hour productivity was negative. 


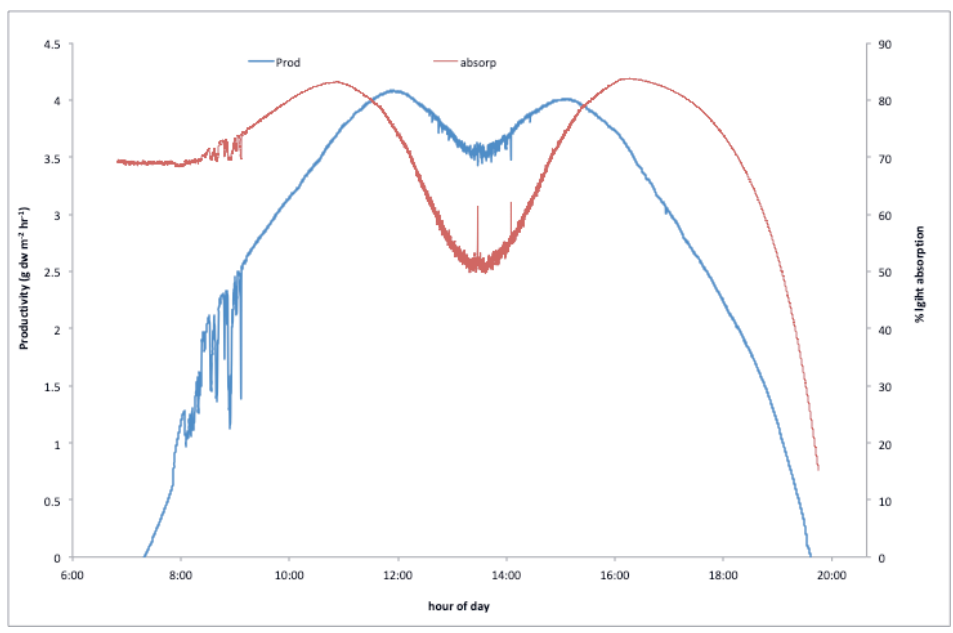

Fig. 4-10 Light absorption (abs) and productivity rate ( $\left.P R_{\text {sim }}\right)$ on a sunny summer day (09.09.12)

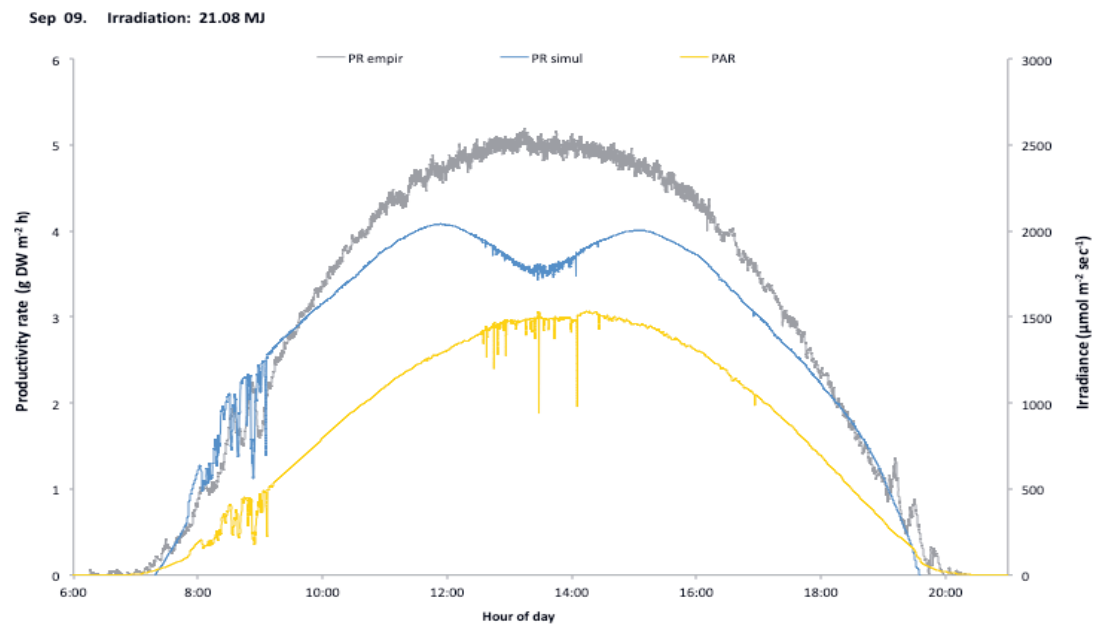

Fig. 4-11 Productivity rate (simulated and empirical) on a cloudless summer day (09.09.2012) 


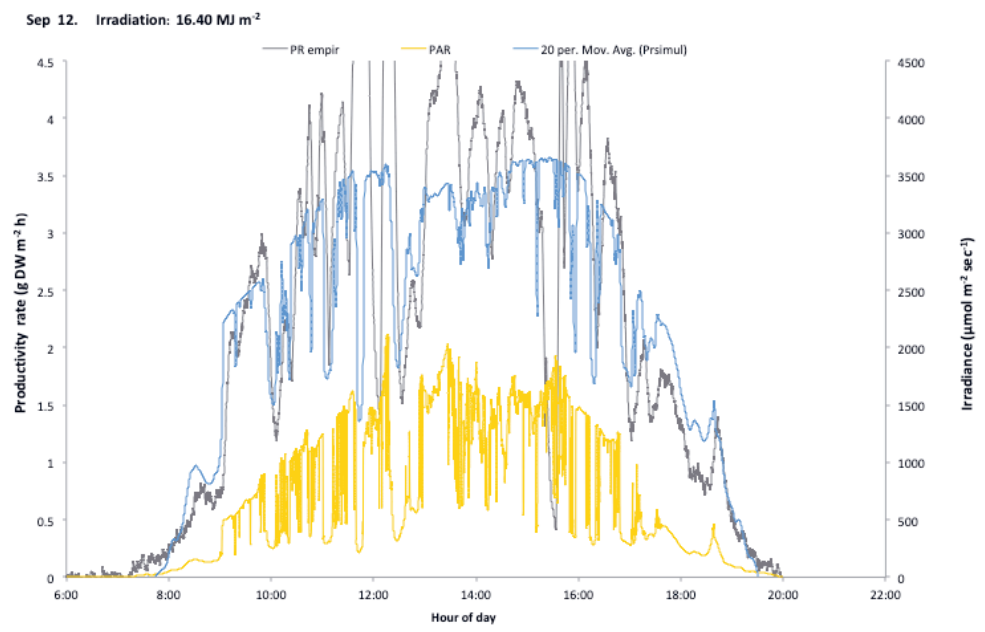

Fig. 4-12 Productivity rate (simulated and empirical) on a summer day with strongly variable cloud cover (12.09.2012). Note: the large drop in the empirical productivity occurring at 15 hours is due to a depressurization of the plant. Note also that the calculated values have been smoothed (gliding average) for clarity in comparison.

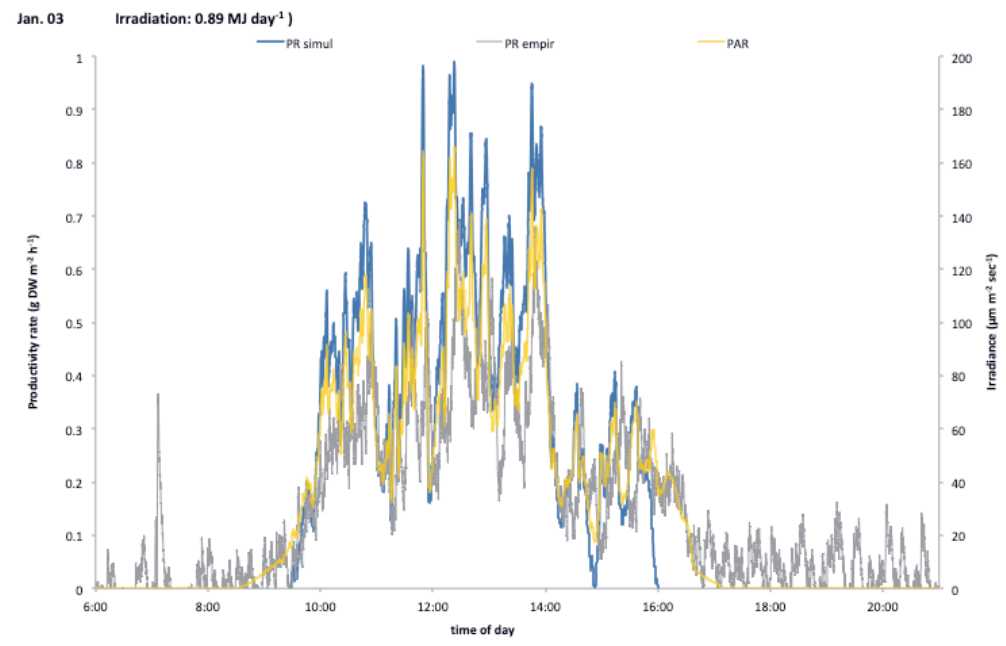

Fig. 4-13 Productivity rate (simulated and empirical) on a cloudy winter day (Jan. 03) with very low irradiation $\left(0.89 \mathrm{MJ} \mathrm{m}^{-2}\right)$ 


\section{Comparison of simulated and empirical productivity}

Table 4-4 Simulated and empirical productivity and photosynthetic efficiency and photosynthetic efficiency (PE) values for the ProviApt reactor at 3 days: 09/09: a cloudless summer day; the 12/09: a summer day with variable cloud cover and 03.01, a winter day with variable cloud cover. Rows with simulated values indicated with "SIM".

\begin{tabular}{|c|c|c|c|c|c|}
\hline Date & unit & & 09/09 & $12 / 09$ & $03 / 01$ \\
\hline Hours of light (daytime) & hours & & 13.67 & 13.12 & 8.48 \\
\hline Irradiation & $\mathrm{MJ} \mathrm{m}^{-2}$ & & 21.08 & 16.40 & 0.89 \\
\hline Reactor biomass concentration & $\mathrm{g} \mathrm{DW} \mathrm{L}^{-1}$ & & 3.77 & 3.21 & 2.23 \\
\hline Productivity (daytime hours) & g DW m ${ }^{-2}$ & & 41.46 & 29.72 & 1.93 \\
\hline Productivity (daytime hours) & g DW $\mathrm{m}^{-2}$ & SIM & 35.35 & 27.31 & 2.35 \\
\hline Productivity (24 hours) & g DW m ${ }^{-2}$ & & 31.79 & 23.81 & -0.04 \\
\hline PE (daytime hours) & $\%$ & & 3.78 & 3.48 & 4.17 \\
\hline PE (daytime hours) & $\%$ & SIM & 3.22 & 3.20 & 5.07 \\
\hline PE (24 hours) & $\%$ & & 2.89 & 2.79 & -0.09 \\
\hline
\end{tabular}

\subsection{Conclusion}

Based on daytime productivity and general trends of the simulated and empirical productivity rate in Fig. 4-11, Fig. 4-12 and Fig. 4-13, one may conclude that it is possible to predict the productivity of the reactor, with the limitations described $i$ the current work, from a simple steady state growth rate function and the two easily measured parameters, horizontal irradiation and reactor temperature.

The productivity of the reactor corresponds to photosynthetic efficiencies around $3-5 \%$ of the total light spectrum (Table 4-4).

The model can be used as a practical tool to improve reactor output by optimizing the parameters biomass concentration, panel aspect ratio, reactor position and temperature management under different irradiation scenarios. With modifications of the light absorption model to accommodate other photobioreactor constructions, the simulation method could be used to assist the design and operation of photobioreactors in general. 


\section{Appendix}

To test the growth rate curve fit, a leave-one-out cross validation was performed.

Method: For each of the 16 datapoints, the model was fitted to a training set that left out the datapoint in question. Predicting of the left-out datapoint was done with the fitted coefficients. Relative absolute (root mean square) error and fitted parameters are shown in Table 4-5.

Table 4-5. Relative root mean square error and fitted parameters for the leave-one-out cross validation.

$\begin{array}{lllllll}\begin{array}{l}\text { Training } \\ \text { set } \\ \text { number }\end{array} & \begin{array}{l}\text { Relative root } \\ \text { mean square }\end{array} & \text { Tlet } & \text { Topt } & \beta & \mu \max & \alpha \Theta \\ & & & & & & \\ 1 & 0.16713 & 14.48811 & 22.62061 & 0.59136 & 0.84001 & 0.21240 \\ 2 & 0.21909 & 13.73340 & 22.65739 & 0.69155 & 0.83925 & 0.21240 \\ 3 & 0.26100 & 14.01913 & 22.81659 & 0.68682 & 0.84617 & 0.20030 \\ 4 & 0.12122 & 14.36753 & 22.52173 & 0.58850 & 0.83753 & 0.21814 \\ 5 & 0.13914 & 12.58165 & 23.76063 & 1.22202 & 0.83127 & 0.20774 \\ 6 & 0.22882 & 14.99993 & 17.79938 & 0.10604 & 0.95804 & 0.20355 \\ 7 & 0.35345 & 14.38010 & 22.41407 & 0.52883 & 0.84890 & 0.19405 \\ 8 & 0.17505 & 14.14201 & 22.75630 & 0.69258 & 0.83769 & 0.22156 \\ 9 & 0.03926 & 14.44736 & 22.47107 & 0.53366 & 0.83516 & 0.21084 \\ 10 & 0.15496 & 14.98446 & 20.78861 & 0.18399 & 0.80880 & 0.21268 \\ 11 & 0.00923 & 14.19038 & 22.68388 & 0.65394 & 0.84184 & 0.21248 \\ 12 & 0.23495 & 11.38822 & 23.43287 & 1.94729 & 0.89660 & 0.20618 \\ 13 & 2.02392 & 14.69100 & 23.00236 & 0.43959 & 0.81021 & 0.25265 \\ 14 & 0.05922 & 14.04567 & 22.59675 & 0.72242 & 0.84314 & 0.21132 \\ 15 & 0.00136 & 14.27176 & 22.63428 & 0.61738 & 0.83951 & 0.21264 \\ 16 & 0.08421 & 13.78290 & 22.58282 & 0.85880 & 0.84319 & 0.21555 \\ \text { Full set } & 0.117 & 14.2653 & 22.6332 & 0.62057 & 0.8396 & 0.21268\end{array}$

\section{Result}

The relative root mean square errors of the training sets ranges between $0.4 \%$ and $202.4 \%$ (Table 4-5). The average relative root mean square error of the set of fits is $21.46 \%$, which is to compare with $11.7 \%$ for the fit using the full dataset.

Errors between datapoints and full-set fits/respective $n-1$ training set fits are shown in Fig. 4-14. 


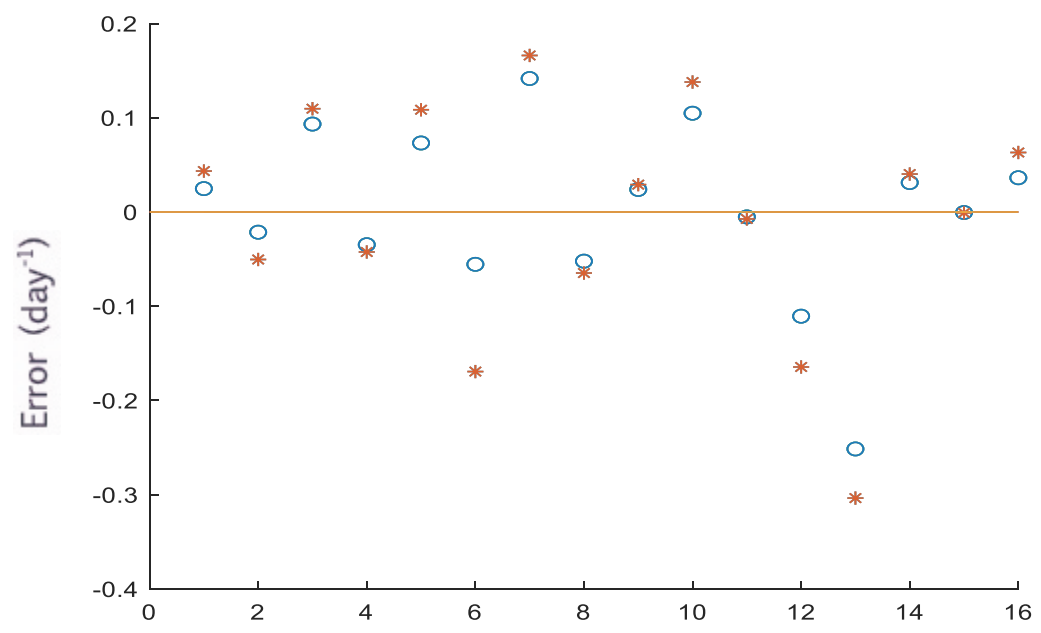

Fig. 4-14 Error between data points and full-set, respective training set curve fits. Symbol: Deviation based on full training set ('o'), deviation based on $n-1$ training set ('*') 


\section{Developing microalgal oil production for an outdoor photobioreactor}

Accepted for publication in Applied Phycology as: Norsker, N.-H., M. Cuaresma, J. d. Vree, P. Uronen, M. J. Barbosa and R. Wijffels Developing microalgal oil production for an outdoor photobioreactor.

\section{Abstract}

In this paper, the preparations are described to develop a production of oil rich microalgal biomass under south European conditions. 10 microalgal species were compared in shake flasks in an incubator for potential for oil production. Potential oil production capacity was assayed as maximum total fatty acid (TFA) concentration and volumetric TFA productivity. TFA concentration ranged from 5 to $40 \%$ DW while TFA productivity rate ranged from 0 to $204 \mathrm{mg}$ TFA L ${ }^{-1}$ day $^{-1}$. To control the oil enrichment process in the outdoor microalgal batch culture, a quadratic equation was proposed, predicting the TFA concentration based on biomass inverse nitrogen ratio. A concentrated substrate was developed to add to sea water, made from natural sea-salt and tap water. 


\section{Introduction}

Scope of the work.

Industrial scale photosynthetic production of microalgal biomass mainly depends on openpond technology whereas photobioreactor technology is applied to produce high-value products and inoculum for pond production. This paper reports on the achievements to develop microalgal oil production for fuel, based on a disposable, soft-sleve tubular photobioreactor. The tubes were placed on the ground, which was partly to avoid the high cost of scaffolding, partly out of necessity as the soft sleeves require full-length support from below. A tube diameter of $6 \mathrm{~cm}$ was selected in accordance with (Acién Fernández et al. 2001). Length of the tubes was $32 \mathrm{~m}$, i.e. the culture would run of 64 meter before returning for degassing.

The current work was carried out in 2010 and 2011, and the purpose was to select a suitable strain to test the tubular microalgal biomass production technology, which was proposed in Norsker et al. (2011) and to develop methodology to enhance the microalgal oil productivity. The study was a technical-economic analysis of various algal production technologies at a Dutch location at 100 ha scale. The technologies compared were open ponds, soft-sleve tubular photobioreactors and vertical, 1 meter tall, flat panels, spaced with 1 meter distance. Out of these 3 technologies, the biomass production costs for the tubular plant were projected as the lowest (€4.15/kg DW). South-west Spain was selected as experimental site because of low annual cloudiness which results in monthly average daily irradiance in the 4 winter months being around $10 \mathrm{MJ} \mathrm{m}^{-2}$ day $^{-1}$ and during the rest of the year 20-25 MJ m${ }^{-2}$ day $^{-1}$ with some days peaking around $30 \mathrm{MJ} \mathrm{m}^{-2}$ day $^{-1}$ whereas summer temperatures rarely exceed 35 degrees. Relatively cool summers, combined with high, sustained irradiance are good conditions to establish microalgal oil production. Huelva University provided access to both laboratory and processing facilities and suitable outdoor space for the later pilot reactor tests at its field research station in Matalascañas. For a soft sleeve tubing photobioreactor, a plane and relatively smooth surface is necessary, and this was provided around the buildings. Batch production technique was applied because it is more simple to use with nutrient limitation than continuous production. Batch production in practice requires a sustained supply of starter culture for 
the reactor but the short production cycles also means lower sanitary demands as predatory or competing organisms are normally not allowed sufficient time to attain troublesome concentrations in the plant.

\section{Oil quantification}

Oil quantification is a problem area in microalgal oil production because of the requirement for rapid feed back on the stage of the product formation. The oil content may be quantified as total lipid, TFA (total fatty acid) or TAG (triacyl glycerol). TAG only includes fatty acids that are esterified with glycerol but not fatty acids in phospholipids and glycolipids that may constitute a significant part of the fatty acids in algae, particularly under nutrient repletion. The lipid fraction contains in addition to fatty acids also chlorophyll, carbohydrate moieties of glycolipids and other non-oil pigments. Discrepancy between lipid and TFA content may be considerable. The cleaned lipid fraction (fatty acid) for example, in the work of Choi et al. constituted $74 \%$ of the crude extract from Scenedesmus obliquus (Choi et al. 1987).

Analysis of microalgal oil content normally is done following Soxhlet, Bligh \& Dyer or Folch extraction methods. These are multistep time consuming methods, often involving large quantities of solvents. (Ahmad et al. 2018). Direct transesterification methods that skip the initial lipid extraction step are faster (Cavonius et al. 2014), but still follow lengthy laboratory protocols. Accelerated solvent extraction, using high pressure extraction cycles is a faster, automated process which is useful for high throughput analytical purposes. Mass spectroscopy (CHNO analysis) may serve to predict fatty acid content but not normally available at microalgal production plants. A relation between total microalgal fatty acid content and C: $\mathrm{N}$ ratio was indeed found in the current study.

Proxy assays may be of help in developing microalgal oil production. Non-destructive carotenoid-chlorophyll ratio, measured on a spectrophotometer was used as an in-direct measure of TFA content in Nannochloropsis oceanica (Solovchenko et al. 2010). In the current work, we developed another proxy assay, the inverse nitrogen quota method to predict and control microalgal fatty acid content. 


\section{Strains for oil production}

The selection of production strain is one of the most critical steps in microalgal oil production development.

\section{Laboratory results}

Oil production has been investigated with a number of strains under laboratory conditions, including Chlorella vulgaris, Chlorella zofingiensis, Nannochloris sp., Scenedesmus obliquus, Dunaliella tertiolecta, Isochrysis galbana, Phaeodactylum tricornutum, Porphyridium cruentum, Chlorococcum littorale, Nannochloropsis oculata, Nannochloropsis sp., Neochloris oleoabundans, Stichococcus bacillaris and Tetraselmis suecica.

These strains were illuminated continuously at $150-190 \mu \mathrm{mol}$ photons $\mathrm{m}^{-2} \mathrm{sec}^{-1}$ in shake flasks and analyzed in terms of fatty acid content and volumetric productivity. The highest fatty acid productivities were exhibited by nitrogen deplete Scenedesmus (360 $\mathrm{mg} \mathrm{L}^{-1}$ day $^{-1}$ ) and Nannochloropsis sp. (238 $\mathrm{mg} \mathrm{L}^{-1}$ day $^{-1}$ ) (Breuer et al. 2012, Benvenuti et al. 2015). Oil rich subpopulations of Chlorococcum littorale were selected with cell sorting and TAG productivity was increased from originally 180 to $340 \mathrm{mg} \mathrm{L}^{-1}$ day $^{-1}$ in flat panel reactors under $400 \mu \mathrm{mol}$ photons $\mathrm{m}^{-2} \mathrm{sec}^{-1}$ irradiance (Cabanelas et al. 2016). TAG content will be less than the TFA content but approaches the TFA content under nitrogen limitation.

\section{Oil production in outdoor photobioreactors}

In Italy, Rodolfi and coworkers maintained a lipid productivity with Nannochloropsis $s p$. in a green wall panel of $204 \mathrm{mg} \mathrm{L}^{-1}$ day $^{-1}$ as a daily average over the first 3 days at a daily average irradiance of $15 \mathrm{MJ} \mathrm{m}^{-2}$ day $^{-1}$. (Rodolfi et al. 2009). The irradiance corresponds to a continuous PAR of $343 \mu \mathrm{mol}$ photons $\mathrm{m}^{-2} \mathrm{sec}^{-1}$.

In Germany, Münkel et al. obtained an average volumetric TFA productivity with Chlorella vulgaris under $\mathrm{N}$ - and P-deprivation of $390 \mathrm{mg} \mathrm{TFA} \mathrm{L}^{-1}$ day $^{-1}$ at a TFA concentration of $44.8 \%$ DW in an outdoor, vertical flat panel reactor during summer (average global irradiance in the period: $15.9 \mathrm{MJ} \mathrm{m}^{-2}$, corresponding to $340 \mu \mathrm{mol}$ photons $\mathrm{m}^{-2} \mathrm{sec}^{-1}$ continuous PAR irradiance (Münkel et al. 2013). 


\section{Comparison between laboratory- and outdoor results}

In terms of volumetric TFA productivity, there is little difference between current best practices: current best laboratory results are $340 \mathrm{mg} \mathrm{TFA}^{-1}$ day $^{-1}$ and best outdoor results are $390 \mathrm{mg} \mathrm{L}^{-1}$ day $^{-1}$.

\section{Oil production enhancement}

The production of microalgal oils generally follows a two-phase pattern with the main biomass production taking place in a nutrient replete stage while enhancement of oil content may follow in a nutrient depleted phase, during which biomass production is reduced or even may become negative. Nitrogen limited, continuous fatty acid production technology has also been proposed but appears not as efficient as batch technology in terms of volumetric productivity. (Klok et al. 2013, Remmers et al. 2017). With continuous production of Neochloris oleoabundans, $79 \mathrm{mg} \mathrm{TFA} \mathrm{L}^{-1}$ day $^{-1}$ was produced under nitrogen limitation whereas nitrogen sufficient productivity was $118 \mathrm{mg}$ (Klok et al. 2013). Continuuus production of nitrogen limited Acutodesmus obliquus resulted a TAG productivity of $0.11 \mathrm{~g} \mathrm{~L}^{-1}$ day $^{-1}$ while batch productivity resulted in $0.23 \mathrm{~g} \mathrm{TAG} \mathrm{L}^{-1}$ day $^{-1}$ (Remmers et al. 2017).

Until the discovery of Nannochloropsis strains that could maintain significant biomass growth for an extended period of time after nitrogen depletion (Rodolfi et al. 2009), nutrient depletion was normally assumed to result in higher fatty acid concentrations but lower productivity than nutrient replete cultures. Breuer found that the main difference between the strains examined was the biomass productivity and capability to sustain biomass production after onset of nitrogen limitation (Breuer et al. 2012).

It is important to notice, that nutrient depletion is not necessarily advantageous in terms of lipid productivity; whereas nutrient deplete Nannochloropsis may produce the highest lipid yields, the efforts associated with a limitation phase in large scale should also be considered. On the other hand, certain extraction protocols require high TAG contents. Fatty acid content and productivity potential, with and without nutrient limitation is therefore important for the evaluation of feasibility of a given strain. 


\section{Current research objectives}

\section{Strains selection}

For the strains selection, fatty acid productivity and fatty acid content in biomass was tested under nitrogen deplete and replete conditions in 10 selected strains. Strains Chlorococcum littorale, Neochloris oleoabundans, Nannochloropsis sp, Phaeodactylum tricornutum, Scenedesmus obliquus and Botryococcus braunii were selected as being among the most frequently investigated strains in microalgal oil studies, whereas Porphyridium cruentum, Tetraselmis suecica and Chlorella sorokiniana are known as prolific biomass producers in large-scale. Scenedesmus sp. (M) was a local spanish isolate that had shown promise in previous outdoor cultivation trials.

A short term, shake flask assay was devised for an orbital shake-flask incubator with start volumes of $100 \mathrm{ml}$, which was sufficient for 2 biomass and fatty acid samples.

\section{Fatty acid enhancement control}

To implement nitrogen limitation in outdoor batch cultures where the nitrogen limitation state of the culture can be difficult to assess and is influenced by the actual weather conditions, we aimed to develop a method to control the nitrogen supply of the culture, the inverse nitrogen quota as a means to predict or control the fatty acid content of the culture. The procedure involves establishing the relation (formula) between the inverse nitrogen quota and the associated fatty acid content in a laboratory assay.

In the outdoor production, the inverse nitrogen quota is calculated as the biomass concentration of the culture, divided by the total assimilated nitrogen of the culture. The algal fatty acid content can then be estimated from the laboratory formula. The formula is species-specific.

\section{Minimum start biomass concentration in the outdoor photobioreactor}

With a batch cultivation and oil enrichment protocol, a steady supply of starter culture must be provided for the photobioreactor. Starter cultures are propagated in successively larger cultivation devices, diluting the culture at transfer 5-10 times and generally inoculate at a minimum biomass density of 5-10 $\mathrm{g} \mathrm{DW} \mathrm{m}^{-2}$ for unspecified strains (Sergejevová et al. 2015). This corresponds to $100-200 \mathrm{mg} \mathrm{L}^{-1}$ in a single layer tubular photobioreactor with 6 
$\mathrm{cm}$ diameter tubes placed closely on the ground. In a commercial production plant, the production of starter cultures is a major effort and plays a pivotal role in the success of the cultivation effort. To estimate the minimum inoculation biomass concentration, we measured the maximum sustainable light exposure of the selected strain in the laboratory from fluorometric quantum yield. From the average light intensity inside the outdoor tubular photobioreactor, a minimum inoculation biomass density was then estimated.

\section{Cultivation medium}

Medium for the outdoor algal production was made from natural seasalt, dissolved in tap water. To supply nutrients, a substrate was developed that could be made concentrated and kept for extended periods without precipitation.

\section{Materials and methods}

\section{Analyses}

Fatty acids were measured in a modified Bligh \& Dyer analysis (Breuer et al. 2013). Total fatty acid productivity, $P t f a_{n}$ from day 0 to day $n$ was calculated according to:

$$
P t f a_{n}=\left(\left(C b_{n} * X t f a_{n}\right)-\left(C b_{0} * X t f a_{0}\right)\right) / n\left[k g m^{-3} d a y^{-1}\right]
$$

With $C b_{n}$ being biomass concentration in biomass on day $\mathrm{n}\left[\mathrm{kg} \mathrm{dw} \mathrm{m}^{-1}\right], C b_{0}$ being biomass concentration in the reactor at the first day of lipid sampling $\left[\mathrm{kg} \mathrm{dw} \mathrm{m}^{-1}\right], X t f a_{n}$ being total fatty acid fraction of biomass at day $\mathrm{n}$ [dimensionless] and Xtfa $a_{0}$ being total fatty acid fraction of biomass at the first day of sampling and $n$ the day number after the beginning of the lipid sampling [dimensionless].

Biomass dry weight was measured in triplicate on pre-dried GF/F fiberglass filters, washing the filters with $0.5 \mathrm{M}$ ammonium formate before drying 24 hours at $95{ }^{\circ} \mathrm{C}$.

Optical density was measured at 735, 680 and $530 \mathrm{~nm}$ on a Genesys UV-10, Thermo Spectronic or, in other situations, on a Bausch \& Lomb Spectronic 20 spectrophotometer. The ratio $\mathrm{OD}_{680} / \mathrm{OD}_{530}$ was taken as an indicator of chlorophyll content in microalgal biomass (Bosma et al. 2008).

Absorption coefficient was measured in a $6 \mathrm{~cm}$ cuvette spectrophotometer equipped with an integrating sphere.

Absorption coefficient $K_{a}$, was calculated according to: 
$K_{a}\left[m^{2} * k g^{-1}\right]=\bar{A}_{400-700 n m} * F * \ln (10) * L^{-1} * C b^{-1}$

(Equation 5-2)

with L: cuvette light path $[\mathrm{m}] ; \mathrm{Cb}:\left[\mathrm{kg} \mathrm{m}^{-3}\right]$ and F: dilution factor for cuvette.

Irradiance was measured with a Licor Li-250 A meter with a $2 \pi$ PAR sensor. In the shake-flask incubator, the sensor was placed at different points between the flasks and directed towards the light source. Irradiance varied between 150 and $205 \mu \mathrm{mol}$ photons $\mathrm{m}^{-2} \mathrm{sec}^{-1}$ with an average close to $200 \mu \mathrm{mol}$ photons $\mathrm{m}^{-2} \mathrm{sec}^{-1}$. Position of the flasks in the incubator were shifted on a daily base to ensure equal irradiance. For the flat panel photobioreactors, used with the inverse nitrogen ratio measurements, light intensity was measured at the surface of the water filled aquarium that the reactors were placed in.

Outdoor irradiance data were kindly provided by the meteorological station at INTA (Instituto Nacional de Técnicas Aeroespaciales), belonging to the Spanish Ministry of Defense, situated at Arenosillo, about $25 \mathrm{~km}$ from the experimental research station in Matalascañas. Data used were hourly global irradiance (in $\mathrm{kJ} \mathrm{m}^{-2}$ ).

Hourly values of $\mathrm{kJ} \mathrm{m}^{-2}$ were converted to average PAR quanta ( $\mu \mathrm{mol}$ photons $\mathrm{m}^{-2} \mathrm{sec}^{-1}$ ) multiplying with the factor: $10^{3} / 3600 * 4.6 * 0.43$ (Slegers et al. 2011).

\section{Strains selection assay}

To select suitable fatty acid producing species, 10 strains were tested in duplicate for fatty acid yield and fatty acid productivity in a $250 \mathrm{~mL}$ conical flasks assay (Table 5-1).

Table 5-1 List of strains included in the strain's selection

\begin{tabular}{lll}
\hline Strain & Designation & Medium \\
\hline Tetraselmis suecica & UTEX LB2286 & Zou (modified) \\
Nannochloropsis oceanica & CCAP 849/10 & Zou (modified) \\
Nannochloropsis sp. & CCAP 211/78 & Zou (modified) \\
Phaeodactylum tricornutum & UTEX 640 & Zou (modified) \\
Porphyridium cruentum & UTEX 161 & Zou (modified) \\
Chlorococcum littorale & NBRC 102761 & Zou (modified) \\
Botryococcus braunii & UTEX 572 & BBM (modified) \\
\hline
\end{tabular}




\begin{tabular}{lll}
\hline Neochloris oleoabundans & UTEX 1185 & BBM (modified) \\
Scenedesmus obliquus & SAG 276-3a & BBM (modified) \\
Chlorella sorokiniana & Not known & BBM (modified) \\
Scenedesmus sp. (local, Matalascañas) & Own isolate & BBM (modified) \\
\hline
\end{tabular}

The algal strains were incubated in Bold's basal medium (Pruvost et al. 2009) or Zou medium (Zou et al. 1999), $\left[\mathrm{NO}_{3}\right]$ in both media set to 5 or $15 \mathrm{mM}$.

Both media were added $1 \mathrm{~mL}$ vitamin solution (composition per liter: B1: 1.2 g; B12 :75 mg; Biotin: $375 \mathrm{mg})$.

The flasks were inoculated at relatively high biomass concentrations $\left(\mathrm{OD}_{530}=0.2\right)$ and incubated in an orbital shake incubator at continuous irradiance of $200 \mu \mathrm{mol}$ photons $\mathrm{m}^{-2}$ $\mathrm{sec}^{-1}$ at 25 deg. and with $5 \% \mathrm{CO}_{2}$ atmosphere maintained in the incubator. In order to assure an even irradiance, the position of the flasks in the incubator were interchanged daily.

Comparing the color of the $5 \mathrm{mM}$ and $15 \mathrm{mM}$ Neochloris-flasks in Fig. 5-1, it is evident that the color of the $5 \mathrm{mM} \mathrm{NO}_{3}$ flasks on the rightmost picture (day 3.71) had started to become pale in comparison with the $15 \mathrm{mM} \mathrm{NO}_{3}$ flasks.
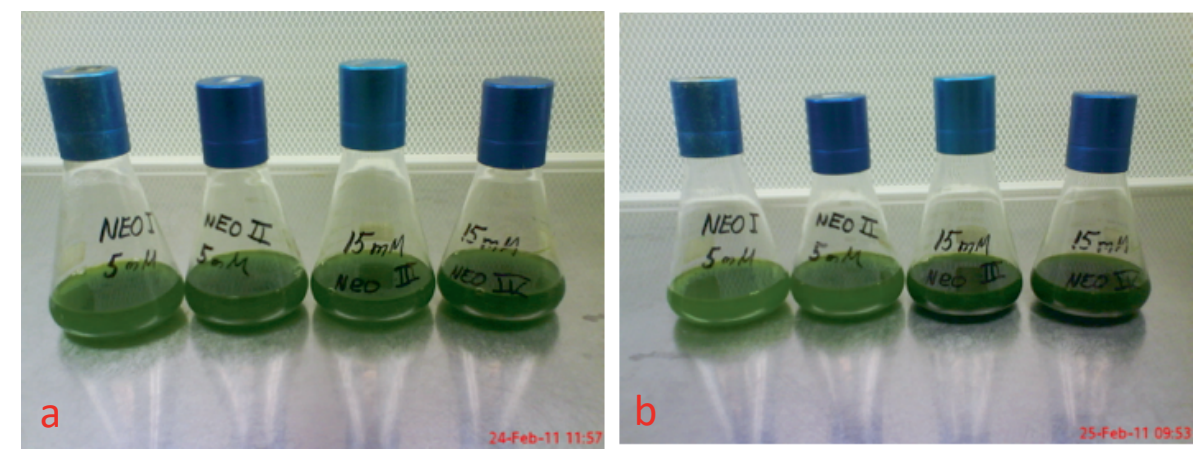

Fig. 5-1 Neochloris oleoabundans flasks photographed a): just before the sampling point (T1) and b): just after the T1, which marks the beginning of the nitrogen limitation phase in the $5 \mathrm{mM} \mathrm{NO}_{3}$-flasks. Notice that the $15 \mathrm{mM}$ $\mathrm{NO}_{3}$ flasks are considerably darker than the $5 \mathrm{mM} \mathrm{NO}_{3}$ flasks

These pigment changes were followed spectrophotometrically.

For each strain, two sampling points were determined on the basis of the $\mathrm{OD}_{530}$ value and $O D_{680} / O D_{530}$ ratio: $T 1$, the first point at which the $O D_{530}$ trend and the $O_{680} / O D_{530}$ trend have started to fall in the $5 \mathrm{mM} \mathrm{NO}_{3}$ flask and nitrogen limitation of could be assumed to 
have begun and $T 2$, a point before the $\mathrm{OD}_{530}$ trend and $\mathrm{OD}_{680} / \mathrm{OD}_{530}$ trend would be expected to wane off in the $15 \mathrm{mM} \mathrm{NO}_{3}$ flasks. In this manner, the $5 \mathrm{mM} \mathrm{NO}_{3}$ cultures could be assumed to exhibit nitrogen deplete fatty acid formation and the $15 \mathrm{mM} \mathrm{NO}_{3}$ a nitrogen replete fatty acid formation rate between $\mathrm{T} 1$ and $\mathrm{T} 2$. As examples of the trends, the trends for Neochloris oleoabundans and Chlorococcum littorale are shown in Fig. 5-2 and Fig. 5-3, respective.

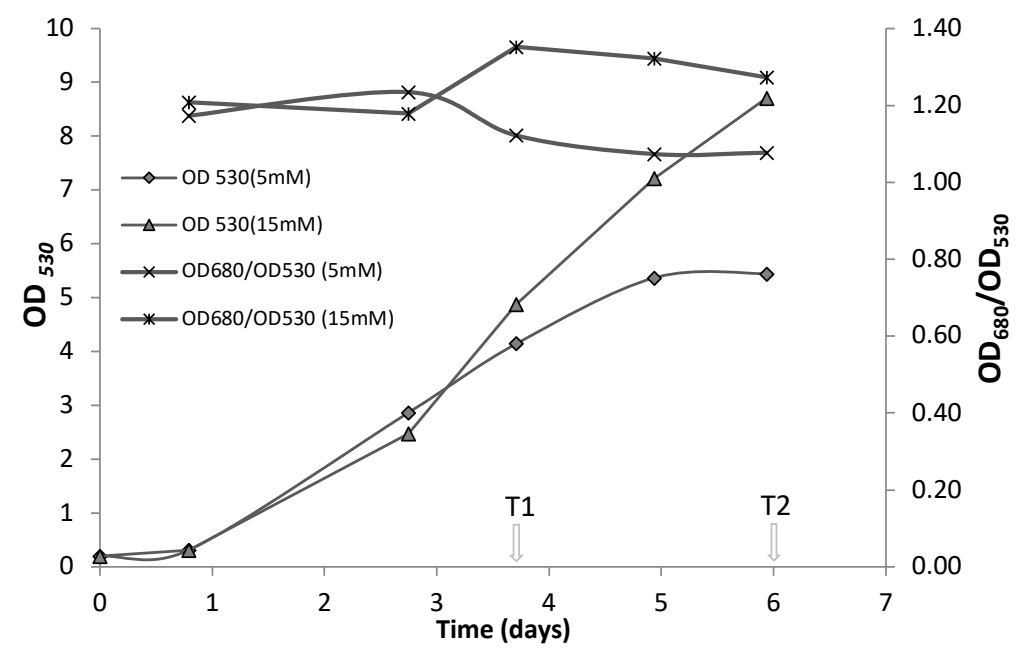

Fig. 5-2 TFA production in Neochloris oleoabundans. T1 and T2 sample points indicated with arrows. FA productivity measurement period: $T 2-T 1$ : $5.94-3.71=2.23$ days 


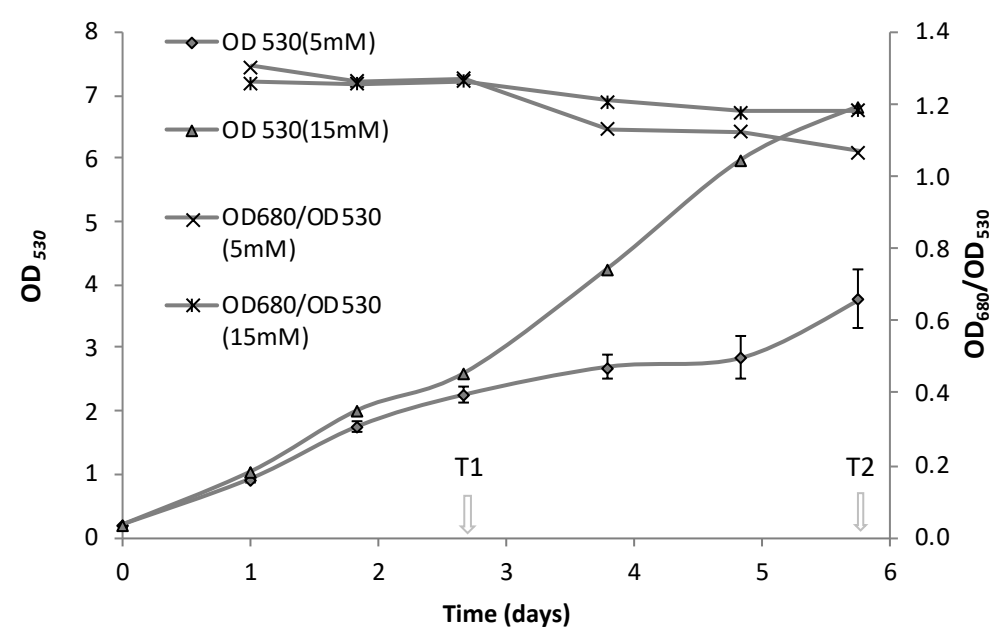

Fig. 5-3 TFA production in Chlorococcum littorale. T1 and T2 sample points indicated with arrows. T2 - T1: 5.75-2.67 = 3.08 days.

It can be noted from the figure that the ratio $O D_{680} / O D_{530}$ changes less during the TFA productivity measurement period than with Neochloris oleoabundans.

\section{Growth medium}

A concentrated and storage-stable substrate was designed for addition to natural sea water in order to make growth medium for high biomass density cultures of marine algae at large scale.

The use of the substrate with natural seawater allowed sodium, calcium and magnesium to be excluded from the substrate, which resulted in a long-term stable (non-precipitating) substrate. Recipe in Table 5-2 .

$20 \mathrm{~mL}$ of substrate per liter of final medium were tested with a batch culture of Neochloris oleoabundans and sufficed for a biomass concentration of $7 \mathrm{~g} \mathrm{DW} \mathrm{L^{-1 }}$. In the large-scale algal production, biomass concentration was kept below $3 \mathrm{~g} \mathrm{DW} \mathrm{L}^{-1}$. 
Table 5-2 Composition of the medium, used to propagate and produce the microalgal species in the study

\begin{tabular}{|c|c|c|}
\hline Trace mineral stock & $\begin{array}{r}\text { Resulting } \\
\text { concentration } \\
\text { in medium }\end{array}$ & Per liter stock solution \\
\hline $\mathrm{Na}_{2} \mathrm{EDTA}$ & $282 \mu \mathrm{M}$ & $45 \mathrm{~g}$ \\
\hline $\mathrm{FeSO}_{4} \cdot 7 \mathrm{H}_{2} \mathrm{O}$ & $108 \mu \mathrm{M}$ & $30 \mathrm{~g}$ \\
\hline $\mathrm{MnCl}_{2} \cdot 2 \mathrm{H}_{2} \mathrm{O}$ & $11 \mu \mathrm{M}$ & $1.71 \mathrm{~g}$ \\
\hline $\mathrm{ZnSO}_{4} \cdot 7 \mathrm{H}_{2} \mathrm{O}$ & $2.3 \mu \mathrm{M}$ & $0.66 \mathrm{~g}$ \\
\hline $\mathrm{Co}\left(\mathrm{NO}_{3}\right)_{2} \cdot 6 \mathrm{H}_{2} \mathrm{O}$ & $0.24 \mu \mathrm{M}$ & $70 \mathrm{mg}$ \\
\hline $\mathrm{CuSO}_{4} \cdot 5 \mathrm{H}_{2} \mathrm{O}$ & $0.1 \mu \mathrm{M}$ & $24 \mathrm{mg}$ \\
\hline $\mathrm{Na}_{2} \mathrm{MoO}_{4} \cdot 2 \mathrm{H}_{2} \mathrm{O}$ & $1.1 \mu \mathrm{M}$ & $242 \mathrm{mg}$ \\
\hline
\end{tabular}

$\mathrm{pH}$ adjusted to about 4

Deion. Water

ad tot vol. $1 \mathrm{~L}$

\begin{tabular}{lrr}
\hline Substrate & $\begin{array}{r}\text { Resulting } \\
\text { concentration } \\
\text { in medium }\end{array}$ & per liter substrate \\
& $50 \mathrm{mM}$ & $212 \mathrm{~g}$ \\
$\mathrm{NaNO}_{3}$ & $1.7 \mathrm{mM}$ & $11.5 \mathrm{~g}$ \\
$\mathrm{KH}_{2} \mathrm{PO}_{4}$ & $173 \mu \mathrm{M}$ & 3 \\
$\mathrm{Na}_{2} \mathrm{EDTA}$ & & $50 \mathrm{ml}$ \\
$\mathrm{Trace}$ mineral stock & & \\
pH adjusted to 7.5-7.6 & & ad tot vol. $1 \mathrm{~L}$ \\
Deion. Water & &
\end{tabular}

\section{Medium}

Substrate

Natural seawater
$20 \mathrm{ml}$

ad tot vol. $1 \mathrm{~L}$ 
Table 5-3 P-normalized elemental ratios in the medium, relative to equivalent cell quotas for average phytoplankton in (Ho et al. 2003)

\begin{tabular}{ll}
\hline Element & Ratio \\
\hline $\mathrm{P}$ & 1.00 \\
$\mathrm{~N}$ & 1.84 \\
$\mathrm{Fe}$ & 8.47 \\
$\mathrm{Mn}$ & 1.70 \\
$\mathrm{Zn}$ & 3.55 \\
$\mathrm{Co}$ & 0.74 \\
$\mathrm{Cu}$ & 0.15 \\
$\mathrm{Mo}$ & 19.61 \\
\hline
\end{tabular}

We designed medium with a high N:P ratio (30) in order to enable high biomass concentrations when growing Phaeodactylum tricornutum which requires a N:P ratio ranging from 24 to 45 , depending on growth rate, ranging from to 0 to 0.8 day $^{-1}$ (Terry et al. 1985).

The elemental composition ratio of the medium to that of average phytoplankton (Ho et al. 2003) is given in Table 5-3. The ratios were normalized with respect to phosphorous.

Elements with strong deviation from natural phytoplankton content included Molybdenum (Mo), 19.61 times higher), Fe (8.47 times higher) and $\mathrm{Cu}: 0.15$ times. Mo was increased to avoid interference in nitrogen assimilation from Mo limitation as Mo is a cofactor in nitrate reductase and limitation can interfere in carbon partitioning under nitrate depletion (Guerra et al. 2013). Fe was increased to avoid iron limitation due to oxidation and precipitation and $\mathrm{Cu}$ was reduced due to low Cu-tolerance in Phaeodactylum.

For introduction of nitrogen limitation in the cultivation, the $\mathrm{NaNO}_{3}$ was reduced without modifying the other medium components.

\section{Inverse nitrogen quota}

4 flat panel photobioreactors (cuvettes from Photon Systems FMT 150) with a light-path of $2 \mathrm{~cm}$ and a working volume of $400 \mathrm{~mL}$ were used. The photobioreactor units were placed in pairs in two aquaria and operated in batch mode.

Mixing of the cultures was done by aeration. A mixture of air and carbon dioxide was supplied to the culture broth through a metal tube with holes placed at the bottom. Before entering the system, the flow rate of both gases was set with two independent mass flow controllers to obtain a final $\mathrm{CO}_{2}$ concentration of $2 \% \mathrm{v} / \mathrm{v}$. The gas was humidified to 
minimize evaporation inside the reactors. The total gas flow rate was $1.12 \mathrm{~L} \mathrm{~min}^{-1}$ and it was distributed equally between the four photobioreactor units.

Temperature was controlled at 25 degrees with a water bath with an external recirculation loop, connected to the aquaria. The 4 photobioreactor units were placed in the aquaria, which were connected to the recirculation loop. An external temperature sensor connected to the water bath was placed in one of the aquaria to maintain the temperature according to the setpoint.

Each aquarium contained 2 photobioreactor units with the two different initial nitrogen concentrations (10 $\mathrm{mM}$ and $2.5 \mathrm{mM}$ of nitrate).

Two panels with fluorescent lamps were used to provide continuous illumination of the photobioreactors at an incident intensity of $120 \mu \mathrm{mol}$ photons $\mathrm{m}^{-2} \mathrm{~s}^{-1}$.

Light input, temperature, and air and $\mathrm{CO}_{2}$ flow were set at the beginning of the cultivation and the flow of $\mathrm{CO}_{2}$ was adjusted to set $\mathrm{pH}$ around 7 .

The cultures were followed for 15 days ( $2.5 \mathrm{mM}$ nitrate cultures) and 22 days (10 mM nitrate cultures). The $2.5 \mathrm{mM}$ nitrate-cultures were running out of residual nitrate after about 4 days and the $10 \mathrm{mM}$ nitrate-cultures after about 15 days.

Residual nitrate was followed in the medium at several points throughout the test, in a photometric assay with two different reaction kits with a dynamic range of 0.3-30 $\mathrm{mg} \mathrm{NO}_{3}-$ $\mathrm{N} \mathrm{L}^{-1}$ and $0.01-0.5 \mathrm{mg} \mathrm{NO}_{3}-\mathrm{N} \mathrm{L}^{-1}$, respectively.

$\mathrm{pH}$ control was done indirectly via carbon dioxide addition. The fixed $\mathrm{CO}_{2}$ concentration supplied to the systems resulted in a stable $\mathrm{pH}$ in the cultures.

\section{Calculation of inverse nitrogen quota:}

$r_{N}^{-1}(t)=\frac{C_{b}(t)}{\left(N_{0}-N_{t}\right)} \quad\left[g * \mathrm{mmol}^{-1}\right]$

with $C_{b}(t)\left[g D W L^{-1}\right]$ denoting culture biomass concentration at time $t$, and $N_{o}$ and $N_{t}[\mathrm{mM}]$ denoting culture initial- and residual nitrogen concentration, respectively. Nitrogen added with the inoculum is also included in $N_{0}$. 


\section{Mass spectroscopy}

Elemental analysis of dried algal biomass samples from the inverse nitrogen quota assay was performed by a carbon/hydrogen/nitrogen elemental analyzer. Oxygen content was calculated by difference.

\section{Average irradiance}

$I_{a v}$, average irradiance in the tubular reactor was calculated from the integrated LambertBeer formula:

$I_{a v}=\frac{I_{0}}{p * C_{b} * K_{a}}\left(1-\exp \left(-p * C_{b} * K_{a}\right)\right)$

(Equation 5-4)

Surface irradiance, $\left(I_{0}\right)\left[\mu \mathrm{mol}\right.$ photons $\left.\mathrm{m}^{-2} \mathrm{sec}^{-1}\right]$ was taken as the irradiance on the horizontal projection of the tubes. $p[m]$, the photic depth was taken as the tube diameter. $C_{b}\left[\mathrm{~g} \mathrm{~m}^{-3}\right]$ is biomass concentration and $K_{a}\left[\mathrm{~m}^{2} \mathrm{~kg}^{-1}\right]$ is the light absorption coefficient for the algal biomass.

The absorption coefficient, $K_{a}$ was determined in a spectrophotometer equipped with an integrating sphere for low light adapted Neochloris.

\section{Results and discussion}

\section{Strains selection}

The results of the strains selection are summarized in Fig.4, where total fatty acid productivity and fatty acid concentration over the period T1-T2 is shown for all 10 strains. From Fig. 5-4, it is obvious that N-depleted Nannochloropsis sp. exhibited both the highest total fatty acid formation rate ( $210 \mathrm{mg} \mathrm{TFA} \mathrm{L}^{-1}$ day $\left.^{-1}\right)$ and total fatty acid content (40\% DW). Phaeodactylum showed roughly the same TFA content but lower TFA formation rate (120 mg TFA L ${ }^{-1}$ day $^{-1}$ ) in the $\mathrm{N}$-depleted flasks. Neochloris, on the other hand, exhibited approximately the same TFA productivity as Nannochloropsis, but lower TFA contents. Chlorococcum exhibited slightly lower TFA productivties than Nannochloropsis but much lower TFA contents. 


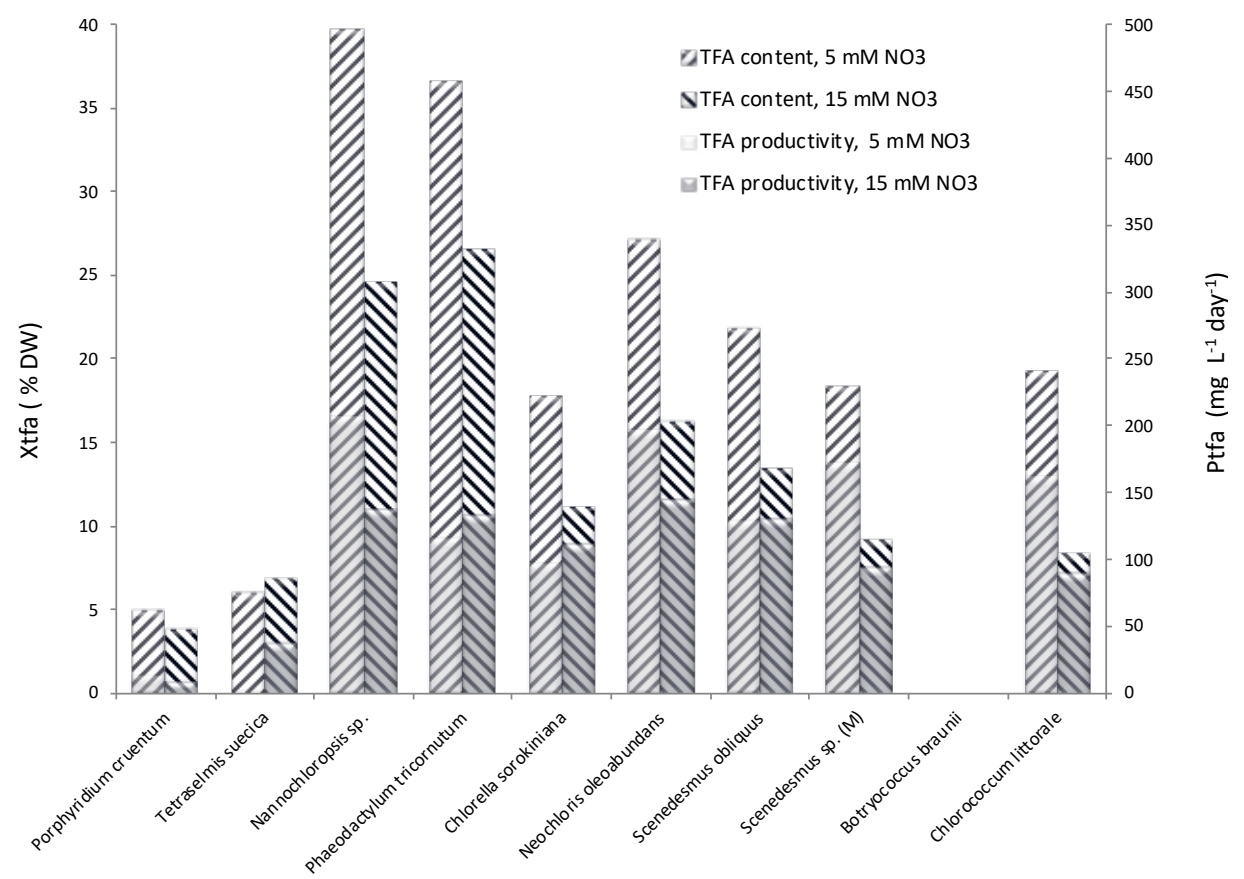

Fig. 5-4 Fatty acid productivity (shaded signature) and fatty acid content (hatched signature) in all 10 strains during short-term from T1 to T2. The $5 \mathrm{mM}$ nitrate flasks are assumed to be nitrogen deplete, whereas the $15 \mathrm{mM}$ nitrate flasks are assumed to be nitrogen replete. No values for Botryococcus braunii are shown as the cultures failed to grow during the incubation

From laboratory fatty acid production tests, Nannochloropsis resulted as the best strain.

Selecting a strain for outdoor fatty acid production, other characteristics may also be considered, such as temperature and light tolerance and amenability for downstream processing. During the strain's selection study, Nannochloropsis was noticed to be sensitive (tended to bleach) at an irradiance over $200 \mu \mathrm{mol}$ photons $\mathrm{m}^{-2} \mathrm{sec}^{-1}$. Phaeodactylum was deselected for its low TFA productivity at nitrogen depletion. Consequently, Neochloris was selected as the most promising strain for further testing. Chlorococcum also was selected as a reasonably good fatty acid producer both in terms of productivity and contents under nitrogen limitation. 


\section{Minimum inoculation biomass density}

To determine the minimum inoculation biomass density, the apparent maximum permissible irradiance was determined in a dilute $\left(C_{b}\right.$ about $\left.0.1 \mathrm{~g} \mathrm{DW} \mathrm{L}^{-1}\right)$, continuously irradiated $400 \mathrm{ml}$ flat panel laboratory turbidostat culture. This was done by stepwise raising irradiance and temperature (for one day) until the fluorescence-based quantum yield and dilution rate were approaching 0 (Table 5-4).

From Table 5-4, it appears that both dilution rate and quantum yield is strongly reduced at $300 \mu \mathrm{mol}$ photons $\mathrm{m}^{-2} \mathrm{sec}^{-1}$ at $35^{\circ} \mathrm{C}$ compared to the situation at $210 \mu \mathrm{mol}$ photons $\mathrm{m}^{-2} \mathrm{sec}^{-}$ 1 and it was concluded that the maximum permissible continuous irradiance was $300 \mu \mathrm{mol}$

Table 5-4 Quantum yield and dilution rate in a Neochloris oleoabundans turbidostat culture at a continuous irradiance of 210, respective $300 \mu \mathrm{mol}$ photons $\mathrm{m}^{-2} \mathrm{sec}^{-1}$ and temperatues $30-40^{\circ} \mathrm{C}$. The QY is the light adapted fluorescence based quantum yield as measured by the FMT 150 flat panel photobioreactor from Photic Systems. $D$ is the dilution rate [day ${ }^{-1}$.

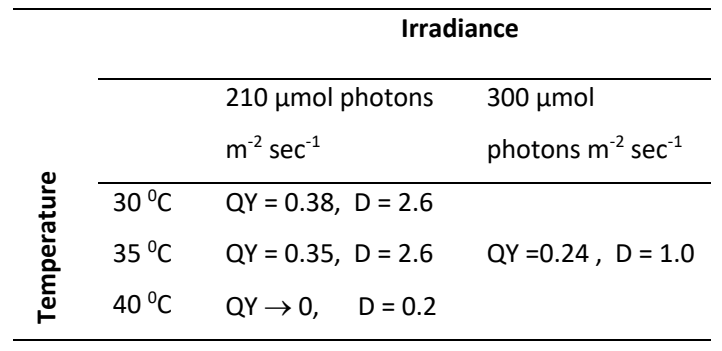
photons $\mathrm{m}^{-2}$ sec $^{-1}$ at $35^{\circ} \mathrm{C}$.

$40{ }^{\circ} \mathrm{C}$ was not sustained.

In a high-biomass density, outdoor tubular photobioreactor, a biomass density was assumed to be a safe minimum (i.e., not resulting in photoinhibition) if the average intensity in the tube $\left(\mathrm{I}_{\mathrm{av}}\right)$ did not exceed $300 \mu \mathrm{mol}$ photons $\mathrm{m}^{-2} \mathrm{sec}^{-1}$ for more than 2 hours at

noon. 


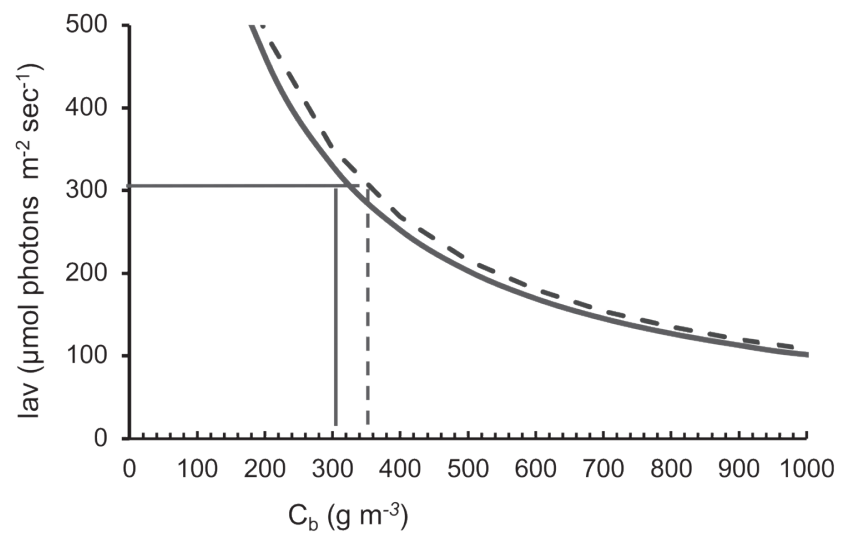

Fig. 5-5 I av depicted against biomass density for two situations: $I_{0}=1277 \mu \mathrm{mol}$ photons $\mathrm{m}^{-2} \mathrm{sec}^{-1}$ (dashed line) and $\mathrm{I}_{0}=1221 \mu \mathrm{mol}$ photons $\mathrm{m}^{-2} \mathrm{sec}^{-1}$ (full line)
In Fig. 5-5, $I_{a v}$ is depicted against $\mathrm{C}_{\mathrm{b}}$ ( biomass concentration) for two irradiance situations: $l_{0}=$ $1277 \mu \mathrm{mol}$ photons $\mathrm{m}^{-2}$ $\mathrm{sec}^{-1}$ which corresponds to the noon irradiance on 06.11.2011 (which is the day with the maximum irradiance in the month of November) and $I_{0}=1221$

$\mu \mathrm{mol}$ photons $\mathrm{m}^{-2} \mathrm{sec}^{-1}$, which is the irradiance limit only exceeded during the solar hours 11 to 13 (Fig. 5-6).

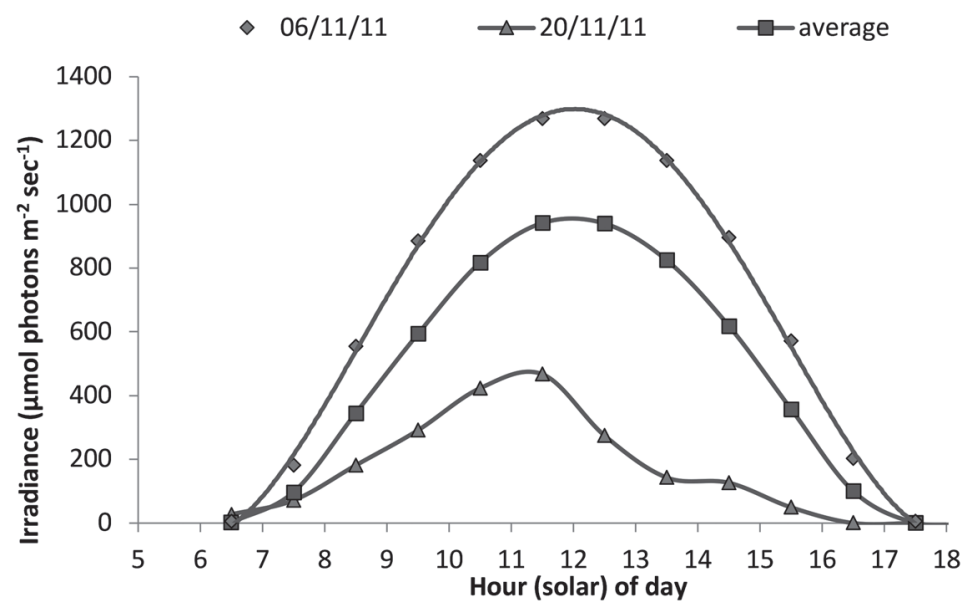

Fig. 5-6 Hourly average irradiance during November 2011. 06.11.2011 was the day with the highest irradiance whereas the 20.11.2011 was the day with the lowest irradiance. Also depicted is the average irradiance profile of the month

Inoculating the $600 \mathrm{~L}$ reactor with a biomass quantity of about $330 \mathrm{~g} \mathrm{~m}^{-3}$, would thus result in an average irradiance exceeding $300 \mu \mathrm{mol}$ photons $\mathrm{m}^{-2} \mathrm{sec}^{-1}$ only during two hours which was judged sustainable, and $330 \mathrm{~g}$ DW was therefore considered the minimum permissible biomass for inoculation of the $600 \mathrm{~L}$ reactor. 
The minimum biomass density found here thus is higher than the $100-200 \mathrm{~g} \mathrm{~m}^{-3}$, recommended by Sergejevová et al (recalculated from 5-10 g DW m²) (Sergejevová et al. 2015).

Keeping the average irradiance below $300 \mu \mathrm{mol}$ photons $\mathrm{m}^{-2} \mathrm{sec}^{-1}$ at all time during a maximum irradiance summer day, would similarly calculated require a minimum biomass concentration of Neochloris of $550 \mathrm{~g} \mathrm{~m}^{-3}$.

\section{Optimization of fatty acid productivity}

For the strains Neochloris oleoabundans and Chlorococcum littorale, fatty acid content was depicted against inverse nitrogen ratio in Fig. 5-7 and Fig. 5-8 and a quadratic polynomium was fitted to the data.

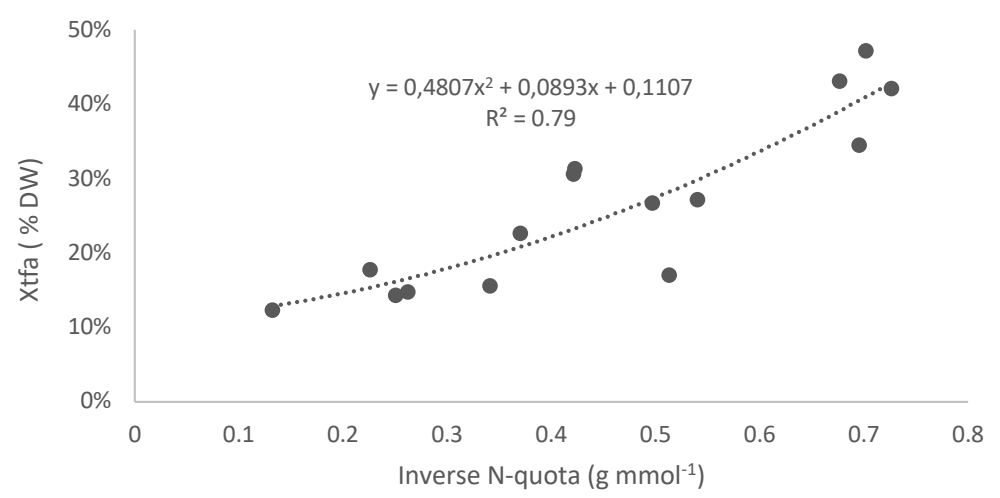

Fig. 5-7 Total fatty acid content in Neochloris oleoabundans depicted against inverse nitrogen cell quota 


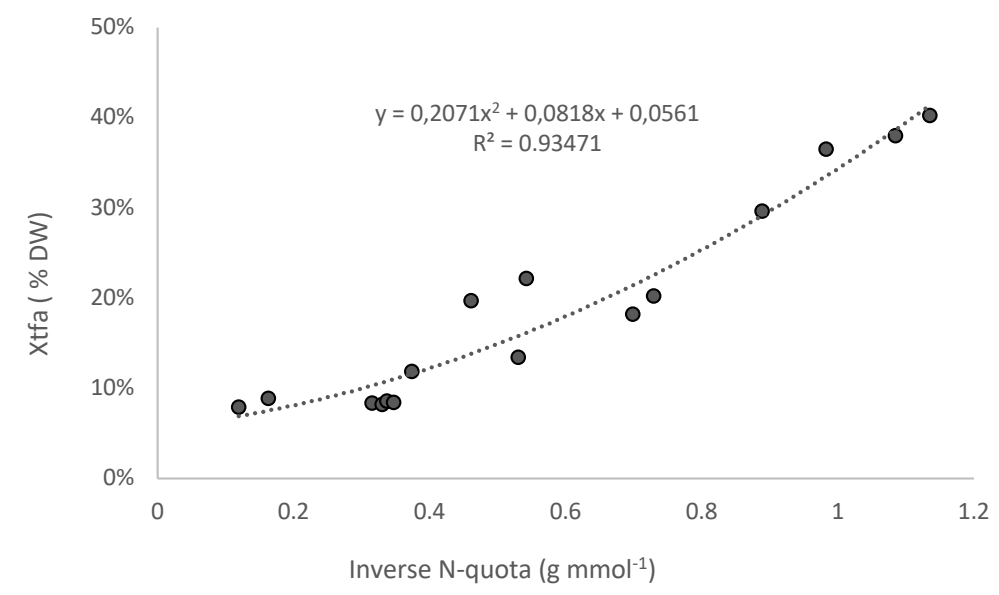

Fig. 5-8 Total fatty acid content in Chlorococcum littorale depicted against inverse nitrogen cell quota

For Neochloris the equation is $y=0.4807 x^{2}+0.0893 x+0.1107$ with y being TFA in \% DW

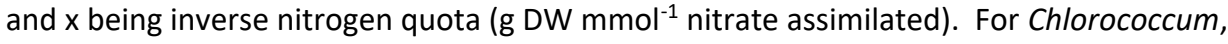
the depiction resulted in $y=0.2071 x^{2}+0.0818 x+0.0561$

For Chlorococcum, the correlation coefficient was 0.93 whereas it was only 0.79 Neochloris. Keeping the values for the 2.5 - and $10-\mathrm{mM}$ series separate, better individual correlation coefficients were obtained (not shown) - but that would no serve the purpose of obtaining a single regression formula.

From the regression line, a target inverse nitrogen ratio could be determined that hypothetically would be associated with a given fatty acid yield in an outdoor culture.

The $\mathrm{C}: \mathrm{N}$ ratio as measured by elemental analysis of the biomass (Chlorococcum littorale) was proportional with the fatty acid content (Fig. 5-9). 


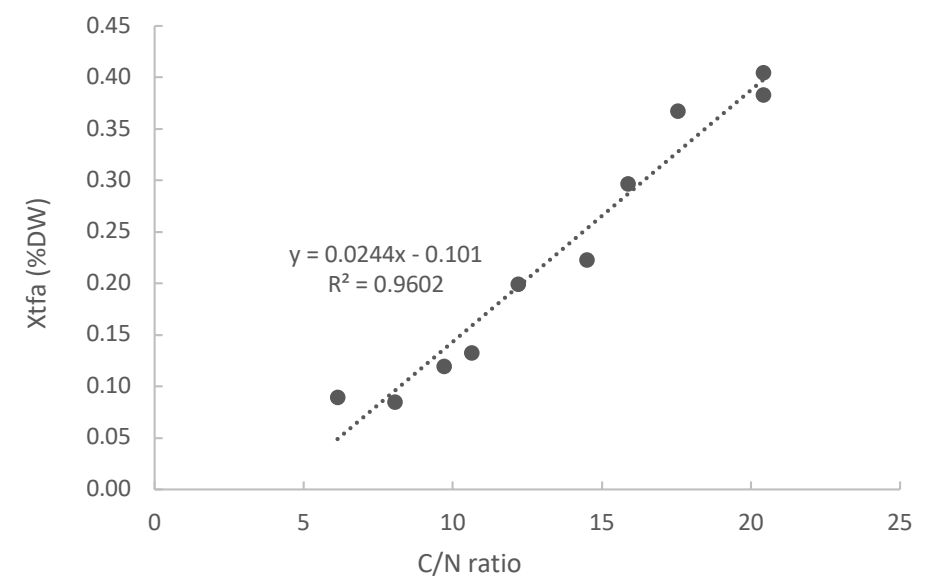

Fig. 5-9 Total fatty acid content in Chlorococcum littorale depicted against elemental C/N ratio
This means

that elemental

analysis of the

biomass could

substitute

direct fatty acid

measurement

but this is little

useful as a

routine

measure

because

elemental analysis is not widely available. Also, the inverse nitrogen quota may be used in a fatty acid enhancement control strategy. The good correlation between fatty acid content and $C / N$ ratio $\left(R^{2}=0.96\right)$ is an indication that both were precise.

\section{Conclusion}

Neochloris oleoabundans and Chlorococcum littorale were selected for testing in a horizontal tubular pilot reactor in southern Spain on the basis potential fatty acid formation rate and fatty acid content under nitrogen deprivation. Nannochloropsis performed better with the strains selection criteria but was not selected because of higher temperature and irradiance sensibility. 


\title{
in an outdoor tubular photobioreactor at
}

\section{pillot scalle}

Submitted to Applied Phycology as: Norsker, N.-H., M. Cuaresma, J. d. Vreea, M. C. Ruiz-Domínguez, M. C. M. García, P. Uronen, M. J. Barbosa and R. Wijffels Neochloris oleoabundans oil production in an outdoor tubular photobioreactor at pilot scale

\begin{abstract}
Oil production was tested with Neochloris oleoabundans in a $6 \mathrm{~m}^{3}$, horizontal soft sleeve tubular reactor from 22 October to 7 November in Matalascañas, southern Spain. Areal biomass productivity during the nitrogen replete phase was $7.4 * 10^{-3} \mathrm{~kg} \mathrm{dw} \mathrm{m}^{-2} \mathrm{day}^{-1}$. Maximum lipid content in the biomass was $39 \%$ and average lipid productivity during the nitrogen depletion phase was $2.0 * 10^{-3} \mathrm{~kg} \mathrm{~m}^{-2}$ day $^{-1}$. Nitrogen depletion of the cultures was carried out in order to enhance fatty acid formation, using the nitrogen ratio in the biomass to predict the fatty acid content. TFA concentration at harvest was $14 \% \mathrm{DW}$, compared to a value of $17 \%$, predicted by the inverse nitrogen ratio. The overall feasibility of the horizontal tubular technology for microalgal oil production, including mixing energy expenditure, was evaluated.
\end{abstract}




\section{Introduction}

Microalgal oil production for transportation fuels received considerable interest during a period with high crude oil prices, roughly from 2005 to 2014. However, several issues of the microalgal production technology remain to be addressed for microalgal oil to become economically feasible for automotive fuels, including basic biomass production technology and oil enhancement methodology. We carried out a full-scale projection study of various algal biomass production techniques in (Norsker et al. 2011) and we examined photobioreactor auxiliary energy requirements in (Norsker et al. 2012).

On the basis of this work, we concluded that the horizontal tubular photobioreactor with disposable soft sleeve-tubes had the best development potential to become a full-scale production technology for microalgal transportation fuels.

The current work describes the pilot scale test of microalgal oil production in an outdoor, horizontal tubular photobioreactor in the South of Spain. The objective was, besides gaining experience with the cultivation technology, to test whether an oil content of $30 \%$ dw could be achieved with the selected strain, measured as total fatty acid fraction of biomass. Tubular photobioreactors are used both for full scale biomass production and to produce starter cultures for open pond production of the algal biomass. The tubular photobioreactor was designed in accordance with the principles in (Norsker et al. 2011) and built in Matalascañas, Huelva region. This test-site was selected because of low cloudiness which results in monthly average daily irradiation most of the year between 20 and $25 \mathrm{MJ} \mathrm{m}^{-2}$ day $^{-1}$, peaking on some summer days around $30 \mathrm{MJ} \mathrm{m}^{-2}$ day $^{-1}$. In December and January average daily irradiation is around $10 \mathrm{MJ} \mathrm{m}^{-2}$ day $^{-1}$. Summer temperatures rarely exceed 35 degrees.

The system was designed for batch mode operation and between campaigns, the polyethylene tubing was discarded, the rest of the system was cleaned and set up with newly propagated starter culture. The system was not attempted hermetically closed. When operating an outdoor photobioreactor as a batch system, inoculation is a recurring challenge depending on system volume and minimum inoculum biomass concentration. Inoculation biomass concentration is a critical as starting the photobioreactor with too low 
biomass concentration results in the culture becoming photo inhibited, possibly irreversibly. It is difficult to find any systematic approach in the literature as to how to start the photobioreactor and to what extent the minimum starter culture concentration depends on strain, state of light adaptation and climatic conditions. A few general recommendations can be found, including scaling up in steps of 5-10, not exposing the culture to full sunlight during the first days and ensure biomass densities between 5 and 10 $g \mathrm{dw} \mathrm{m} \mathrm{m}^{-2}$ (Sergejevová et al. 2015). It is unclear whether the area base with that recommendation should be the total cultivation area or the tube covered area. And furthermore, in order to get a species specific, volumetrically based and more precise assessment, we estimated the minimum inoculum density from the maximum applicable continuous irradiation in a flat panel laboratory photobioreactor. For Neochloris to be able to sustain full sunlight exposure in October at the site, the minimum biomass density was

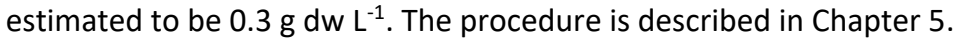

For the pilot scale system to be representative to a full scale installation, the tube length and diameter must be representative.

Tube diameter was chosen as $6 \mathrm{~cm}$ in accordance with (Acién Fernández et al. 2001) as a compromise between pumping costs and irradiation requirements for an $80 \mathrm{~m}$ tube length from degassing to degassing. In this case, tube length was $32 \mathrm{~m}$, resulting in a tube run between degassing events of $64 \mathrm{~m}$. During the run through the transparent tubes, dissolved oxygen concentration builds up. Excessive dissolved oxygen exposure may lead to photo inhibition and tube length is therefore limited by the acceptable dissolved oxygen concentration build-up over the tube length. A good photobioreactor design also depends on an even distribution of the flow in the tubes. A manifold unit was devised that comprised 4 outgoing tubes with the entry port placed centrally and 2 returning tubes on either side. At the end, the tubes were joined in a single, 8-stud manifold. This arrangement ensured - together with suitable dimension of the manifold branches, identical pressure drop and hence culture velocity in the tubes. The photobioreactor had 6 manifold units, amounting to a total tube length of $1536 \mathrm{~m}$. Irradiated volume $4.96 \mathrm{~m}^{3}$ and total volume of plant $6.1 \mathrm{~m}^{3}$. 


\section{Oil production with microalgae.}

The oil content can be quantified as total lipids and TFA (total fatty acids). The lipid extract contains chlorophyll, carbohydrate moieties of glycolipids and other non-oil pigments and by the content of total fatty acids (TFA). Lipid content in microalgae ranges from 5 to over $80 \% \mathrm{dw}$, according to an extensive review of Qiang, considering all strains and growth conditions (Qiang et al. 2008). In mid-Italy, outdoor lipid productivity varied from 1.32 to 2.14 ton ha ${ }^{-1}$ month $^{-1}$ corresponding to 4.4-7.1 $\mathrm{g} \mathrm{m}^{-2}$ day $^{-1}$ with Chlorella vulgaris under nutrient replete conditions (Guccione et al. 2014).

Total fatty acids (TFA) is the most relevant biofuel measure when considering extraction as FAME (fatty acid methyl ester). Lipid composition influences the FAME fraction of the lipid extracts as TAG's (triglycerides) convert $100 \%$ to FAME whereas the glycolipid di-galactosyl diglyceride, for example converts to only 63 \% FAME (Laurens et al. 2012). Discrepancy between lipid and TFA content may therefore be considerable.

Annual average FAME productivity with Nannochloropsis salina in Colorado were $13.1 \mathrm{~m}^{3}$ ha-1 $\mathrm{y}^{-1}$ with a peak summer value of $36.3 \mathrm{~m}^{3} \mathrm{ha}^{-1} \mathrm{y}^{-1}$ (Quinn et al. 2012), corresponding to 3.2 and $8.95 \mathrm{~g} \mathrm{~m}^{-2}$ day $^{-1}$ respectively, calculated using FAME density of $0.918 \mathrm{~kg} \mathrm{~m}^{-3}$ (Quinn et al. 2012).

Under laboratory conditions, light absorption by the biomass can be accurately assessed and the productivity on light can be used to predict outdoor productivity under various climatic conditions. A productivity model, based on a metabolic network of Scenedesmus obliquus and annual solar irradiation data for southern Spain, predicted TAG productivities between 2.1 to $8.8 \mathrm{~g} \mathrm{~m}^{-2}$ day $^{-1}$, the latter with optimal light dilution and use of a starch-less mutant (Breuer et al. 2015).

The production of microalgal oils generally follows a two-phase pattern with the main biomass production taking place in a nutrient replete stage while enhancement of oil content may follow in a nutrient depleted phase, during which biomass production is reduced or even may become negative.

Oil content enhancement normally is done in a batch process. Continuous fatty acid production technology has also been proposed; with Neochloris oleoabundans, TFA 
productivity from 60 to $118 \mathrm{mg} \mathrm{L}^{-1}$ day-1 was obtained with varying degree of nitrogen limitation; the high value obtained with nitrogen replete cultures (Klok et al. 2013) but continuous production appears not as efficient as batch technology in terms of volumetric productivity; in a direct comparison of cultivation technology, batch TAG productivity was $230 \mathrm{mg} \mathrm{L}^{-1} \mathrm{day}^{-1}$ in nitrogen limited Acutodesmus obliquus as opposed to $110 \mathrm{mg} \mathrm{L}^{-1}$ day $^{-1}$ in continuous cultivation. The difference appeared to be related to higher light utilization efficiency with the batch compared to continuous culture (Remmers et al. 2017). The term light utilization efficiency or yield on light (in g lipid/TAG/TFA per mol photons $(P A R))$ is particularly useful for characterizing a given laboratory or field reactor result in terms of what potentially is possible. Remmers and coworkers suggested the maximum realistic TAG yield on light being about $0.53 \mathrm{~g} \mathrm{~mol}^{-1}$, taking TAG content as $50 \%$ dry biomass (Remmers et al. 2018). Values of $0.32 \mathrm{~g} \mathrm{TAG} \mathrm{mol}^{-1}$ were found in lab reactors for low-light cultivated Chlorella zofingiensis at the onset of nitrogen starvation (Mulders et al. 2014). In outdoor flat- and stacked horizontal tubular reactors, TAG yield on light varied between 0.07 and $0.19 \mathrm{~g} \mathrm{~mol}^{-1}$ for Nannochloropsis sp. (Benvenuti et al. 2015).

Recently, a minimum irradiation has been also shown necessary to support high TAG yield in Nannochloropsis gaditana under nitrogen limitation (Janssen et al. 2018). TAG yield on light by $N$. gaditana was maximal $\left(0.09 \mathrm{~g} \mathrm{~mol}^{-1}\right)$ at intermediate biomass specific irradiation, ranging between 11 and $26 \mu \mathrm{mol} \mathrm{sec}^{-1} \mathrm{~g}^{-1}$ (Janssen 2018). The reported productivities are not long-term sustainable, but typically apply to nutrient limited batch cultures over 4-7 days, after which time productivity declines.

With outdoor cultures however, it is difficult to implement such knowledge to optimize oil productivity because of dynamic irradiation, both in terms of day-night cycles and cloudiness.

Furthermore, the degree of nitrogen supply is also critical for the result of the fatty acid enhancement procedure. With prolonged nitrogen starvation, high oil content can be obtained but oil productivity tends to be low because of low biomass productivity. With higher nitrogen supply, oil productivity may be high but at high biomass productivity and low oil content. The overall industrial optimum lies between these two extremes (Adams et al. 2013) and depends on strains characteristics, cultivation conditions, dewatering- and oil extraction technique. Determinining the optimum oil content in the algae is therefore 
arguably complex. In the current work, we aimed at developing an industrially feasible microalgal oil production, leading to a fatty acid biomass content of $30 \%$ with Neochloris oleoabundans. To obtain that, we wanted to test and validate a previously established relationship between inverse nitrogen ratio and fatty acid content in Neochloris (Chapter $5)$.

\section{Materials and methods.}

\section{Analyses}

Lipid

For total lipids content measurement, biomass was recovered and washed with $0.5 \mathrm{M}$ ammonium formate and oven dried at $60{ }^{\circ} \mathrm{C}$. The biomass was ground in a mortar and homogenized by sonication in chloroform-methanol. Total lipids were extracted in an automated Soxhlet device as described in (Ruiz-Domínguez et al. 2015).

Fatty acids were measured in a modified Bligh \& Dyer analysis (Breuer et al. 2013). For the tubular photobioreactor experiments, methylated lipid extracts, produced at the test site in Matalascañas were sent to Wageningen University and analyzed further, according to Breuer et al. Internal standards for all 6 identified fatty acids were included.

Lipid productivity, $P I_{n}$ or total fatty acid productivity, $P t^{\prime} a_{n}$ on the $n^{\prime}$ th day during the final batch phase was calculated according to:

$$
\begin{array}{ll}
P l_{n}=\left(\begin{array}{lll}
\left.\left(C b_{n} * X l_{n}\right)-\left(C b_{0} * X l_{0}\right)\right) / n & {\left[\mathrm{~kg} \mathrm{~m}^{-3} \text { day }^{1}\right]} & \text { Equation 6-1 } \\
P t f a_{n}=\left(\left(C b_{n} * X t f a_{n}\right)-\left(C b_{0} * X t f a_{0}\right)\right) / n & {\left[\mathrm{~kg} \mathrm{~m}^{-3} \text { day }^{1}\right]} & \text { Equation 6-2 }
\end{array}\right.
\end{array}
$$

With $C b_{n}$ being biomass concentration in biomass on day $n, C b_{0}$ being biomass concentration in the reactor at the first day of lipid- or total fatty acid sampling, $X I_{n}$ and $X t f a_{n}$ being lipid fraction and total fatty acid fraction of biomass at day $n$, respective. $X I_{0}$ and $X t f a_{0}$ being lipid- and total fatty acid fraction of biomass at the first day of sampling respective and $\mathrm{n}$ the day number after the beginning of the lipid sampling. 


\section{Biomass}

Biomass dry weight was measured in triplicate on pre-dried GF/F fiberglass filters, washing the filters with $0.5 \mathrm{M}$ ammonium formate before drying $24 \mathrm{~h}$ at $95 \mathrm{deg}$.

\section{Optical density}

Optical density was measured on a Genesys UV-10, Thermo Spectronic or, in other situations, on a Bausch \& Lomb Spectronic 20 spectrophotometer.

\section{Nitrate and phosphate}

Nitrate and phosphate was analyzed in a continuous flow analyzer (Proxima, Alliance). Samples for nitrate, phosphate, lipid and fatty acid content were taken daily, around noon. Biomass dry weight was measured in a separate sample.

\section{Irradiation}

Irradiation measurements were kindly provided by the meteorological station at INTA (Instituto Nacional de Técnicas Aeroespaciales), belonging to the Spanish Ministry of Defense, situated at Arenosillo, about $25 \mathrm{~km}$ from the experimental research station in Matalascañas. Data were given as hourly accumulated horizontal global irradiation, lacc [in $\left.\mathrm{kJ} \mathrm{m} \mathrm{m}^{-2} \mathrm{~h}^{-1}\right]$.

These values were converted to hourly average quantum (PAR) irradiance, $\mathrm{H}_{\text {avg }}\left[\mu \mathrm{mol} \mathrm{m} \mathrm{m}^{-2}\right.$ $\left.\sec ^{-1}\right]$, using the formula in Equation 6-3.

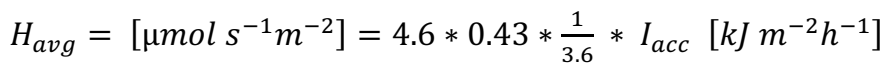

(Slegers et al. 2011).

Irradiance impinging on the reactor tube surface (horizontally projected) was calculated by summing hourly accumulated irradiation over the hours of the day, omitting the time in the evening, where the reactor was completely shadowed by an adjacent laboratory building and the radiance was less than of $200 \mu \mathrm{mol}$ photons $\mathrm{m}^{-2} \mathrm{sec}^{-1}$. Impinging irradiation represented $77-94 \%$ of the horizontal irradiation. 
$I_{a v},\left[\mu \mathrm{mol}\right.$ photons $\left.\mathrm{m}^{-2} \mathrm{sec}^{-1}\right]$ average irradiance in the tubular reactor was calculated from the integrated Lambert-Beer formula. Io [ $\mu$ mol PAR $\left.^{-2} \mathrm{sec}^{-1}\right]$, irradiation impinging on the tube surface, was taken as the irradiation on the horizontal projection of the tubes, $p[m]$, the photic depth was taken as the tube diameter. $C_{b}\left[\mathrm{~kg} \mathrm{dw} \mathrm{m}^{-3}\right]$ is biomass concentration and $\mathrm{K}_{\mathrm{a}}\left[\mathrm{m}^{2} \mathrm{~kg}^{-1}\right]$ is the light absorption coefficient for the algal biomass.

$I_{a v}=\frac{I_{0}}{p * C b * K_{a}}\left(1-\exp \left(-p * C_{b} * K_{a}\right)\right)$ [umol photons $\left.m^{-2} \sec ^{-1}\right]$

Equation 6-4

\section{Photosynthetic efficiency}

Photosynthetic efficiency, PE is the ratio of biomass energy produced during a growth period to the total solar light energy, assimilated by the culture during the period.

In practice, the nutrient replete growth rate, $\frac{\Delta \mathrm{C}_{b}}{\Delta t}$ was multiplied with the estimated calorific content $(\mathrm{H})$ and divided by $I R_{\text {avg }}{ }_{t}^{t+\Delta t}$, the average horizontal irradiation during the period.

This is a simplification, assuming that the light absorption can be taken as the horizontal irradiation, impinging on projection of the tube. Also, it should be noted that $H$, the calorific content, was not measured but estimated on the basis of literature values (Table 6-1) to be characteristic for nutrient replete Neochloris biomass.

$P E=\frac{\Delta C b}{\Delta t} * \frac{H}{I R_{a v g}{ }^{t+\Delta t}}$

Equation 6-5

The calorific content value used in the calculation of photosynthetic efficiency during the nutrient replete phase, was $21 \mathrm{kJg}^{-1}$. To justify the used value, some literature values for algal biomass calorific values, $H$, are presented in Table 6-1. Unfortunately, there are no published values on calorific content of nutrient replete Neochloris, but a figure in the low range of values for Chlorella, would be reasonable to use.

Table 6-1. Calorific content values from literature.

\begin{tabular}{|l|l|l|}
\hline Source & Values $\mathbf{( \mathbf { k J ~ g } ^ { - \mathbf { 1 } } )}$ & Reference \\
\hline Various literature references & $20-23.75$ & (Weyer et al. 2010) \\
\hline Oil rich (lipid $\mathbf{5 0 \%} \mathbf{~ d w}$ ) biomass & 26.9 & (Weyer et al. 2010) \\
\hline Chlorella vulgaris, $\mathbf{5 8} \%$ lipid & 28 & (Scragg et al. 2002) \\
Chlorella vulgaris, $\mathbf{2 8} \%$ lipid & 21 & (Scragg et al. 2002) \\
Chlorella, axenic, lab batch & 22 & (Halfhide et al. 2014) \\
\hline & & \\
\hline
\end{tabular}




\section{Growth medium}

Table 6-2. Composition of the medium, used to propagate and produce the microalgal species in the study.

\begin{tabular}{|c|c|}
\hline Mineral & Concentration \\
\hline $\mathrm{NaNO}_{3}$ & $50 \mathrm{mM}$ \\
\hline $\mathrm{KH}_{2} \mathrm{PO}_{4}$ & $1.7 \mathrm{mM}$ \\
\hline $\mathrm{Na}_{2} \mathrm{EDTA}$ & $173 \mu \mathrm{M}$ \\
\hline $\mathrm{FeSO}_{4}$ & $108 \mu \mathrm{M}$ \\
\hline $\mathrm{MnCl}_{2}$ & $11 \mu \mathrm{M}$ \\
\hline $\mathrm{ZnSO}_{4}$ & $2.3 \mu \mathrm{M}$ \\
\hline $\mathrm{Co}\left(\mathrm{NO}_{3}\right)_{2}$ & $0.24 \mu \mathrm{M}$ \\
\hline $\mathrm{CuSO}_{4}$ & $0.1 \mu \mathrm{M}$ \\
\hline $\mathrm{Na}_{2} \mathrm{MoO}_{4}$ & $1.1 \mu \mathrm{M}$ \\
\hline $\mathrm{NaNO}_{3}$ & $50 \mathrm{mM}$ \\
\hline $\mathrm{KH}_{2} \mathrm{PO}_{4}$ & $1.7 \mathrm{mM}$ \\
\hline $\mathrm{Na}_{2}$ EDTA & $173 \mu \mathrm{M}$ \\
\hline
\end{tabular}

A concentrated and storage-stable substrate was designed for addition to natural sea water in order to make growth medium for high biomass density cultures of marine algae at large scale. The composition of the medium is given in Table 6-2. The complete recipe of the substrate is given in Chapter 5. The use of the substrate with natural seawater allowed sodium, calcium and magnesium to be excluded from the

substrate, which resulted in a long-term stable (non-precipitating) substrate. The recipe had been tested with a batch culture of Neochloris oleoabundans and sufficed for a biomass concentration of $7 \mathrm{~kg} \mathrm{dw} \mathrm{m}^{-3}$. In the large-scale nutrient replete algal production, biomass concentration was kept below $3 \mathrm{~kg} \mathrm{dw} \mathrm{m}^{-3}$. For introduction of nitrogen limitation in the cultivation, the $\mathrm{NaNO}_{3}$ was reduced without modifying the other medium components.

\section{Biomass Propagation}

Cultures were maintained in $250 \mathrm{ml}$ E-flasks in an illuminated ( $25 \mu \mathrm{mol}$ photons $\left.\mathrm{m}^{-2} \mathrm{sec}^{-1}\right)$ orbital shaker with air with $5 \% \mathrm{CO}_{2}$ and temperature control $\left(25^{\circ} \mathrm{C}\right)$. Propagation was done in an 8-panel, semi-continuously operated reactor, see Fig. 6-1. The dimensions of the panels were $50 \times 50 \times 1 \mathrm{~cm}$, filled to a volume of about $1.7 \mathrm{~L}$. The panels were made by the Belgian chemical company Proviron from clear polypropylene film and front- and back sides were welded together creating vertical channels. Each 


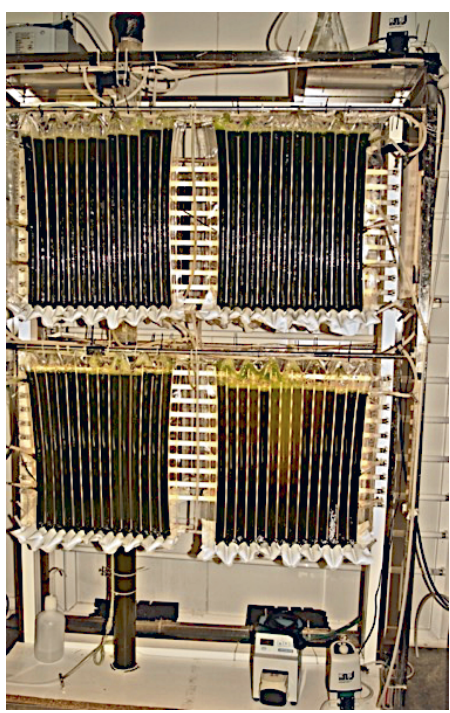

Fig. 6-1 Propagation reactor with 8 Proviapt panels (4 of them behind fluorescent tube banks) channel was sparged from a chamber below the channels. The panels were suspended on both sides of a bank of 20 fluorescent T5 tubes (54 W) with electronic ballasts at a distance of approximately 20 $\mathrm{cm}$ from the panels.

Maximum irradiance at the surface of the panels was $450 \mu \mathrm{mol}$ photons $\mathrm{m}^{-2} \mathrm{sec}^{-1}$, normally operated at 200-300 $\mu \mathrm{mol} \mathrm{m}{ }^{-2} \mathrm{sec}^{-1}$.

A diaphragm pump (500 $\mathrm{W}$ ) recycled the aeration gas.

A fixed flow of $\mathrm{CO}_{2}$ and compressed air from mass

flow controllers was sufficient to control $\mathrm{pH}$.

About $25 \%$ of the culture was daily exchanged with fresh medium. In total, about 3-4 liters were daily harvested at a biomass concentration of 2-4 $\mathrm{kg} \mathrm{dw} \mathrm{m}^{-3}$ and stored in the dark at 4 deg until use. Cultures

typically retained their productivity for 2-3 weeks (data not shown). The reactor was operated aseptically and unialgal cultures were produced.

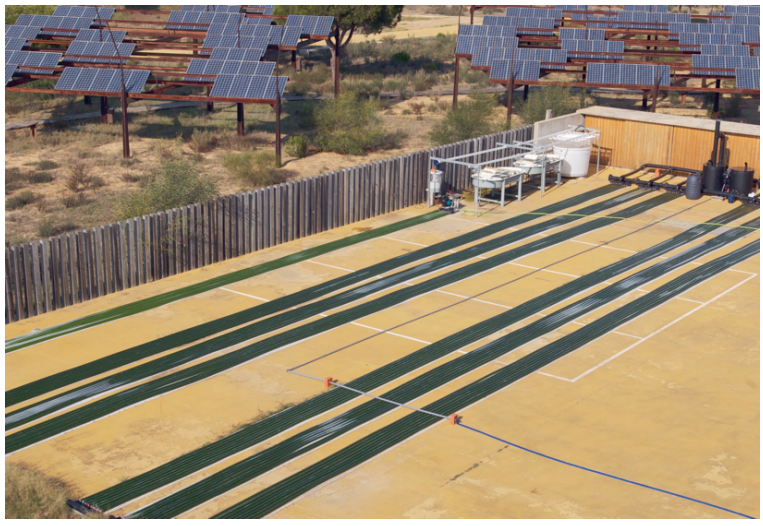

Fig. 6-2 The two out-door photobioreactors on the 27.11.2011. In front, the 6 lanes belonging to the $6 \mathrm{~m}^{3}$ reactor; in the back the single lane of the $600 \mathrm{~L}$ reactor, used for producing starter culture for the $6 \mathrm{~m}^{3}$ reactor. The tube crossing the 3 front lanes is the harvest line
The 600 literphotobioreactor

The 600-liter photobioreactor was a soft-sleeve tubular photobioreactor with a single, $24 \mathrm{~m}$ long lane and a single degasser-buffer tank. In Fig. $6-2$, it is adjacent to the $6 \mathrm{~m}^{3}$ reactor, near the fence. The manifold system was similar to the $6 \mathrm{~m}^{3}$ reactor. The circulation pump was a $2.3 \mathrm{~kW}$

ESPA, $3 \times 380$ V, 2650 rpm swimming pool pump. Normally, the ESPA pump was operated 
at a power supply of $0.9 \mathrm{~kW} \mathrm{~m}^{-3}$, effectively subjecting the biomass to a much higher shear stress than the circulation pump in the $600 \mathrm{~L}$ reactor. Degassing was carried out by falling film: in the degasser-buffer tank, the inlet ejected vertically up-wards with a $75 \mathrm{~mm}$ tube, falling down as an umbrella-shaped sheet of fluid. This degassing method was found more effective and energy-economic than air sparging (data not shown).

\section{The $6 \mathrm{~m}^{3}$ photobioreactor}

\section{Design}

The tubular photobioreactor was designed by the authors and built by the Dutch engineering company Paques and encompassing a flow distribution- and degassing system for 6 lanes of 8 soft PE tubes, the 4 central tubes being exit tubes and two peripheral tubes on either side being return tubes. At the end of each lane, all 8 tubes were connected in a

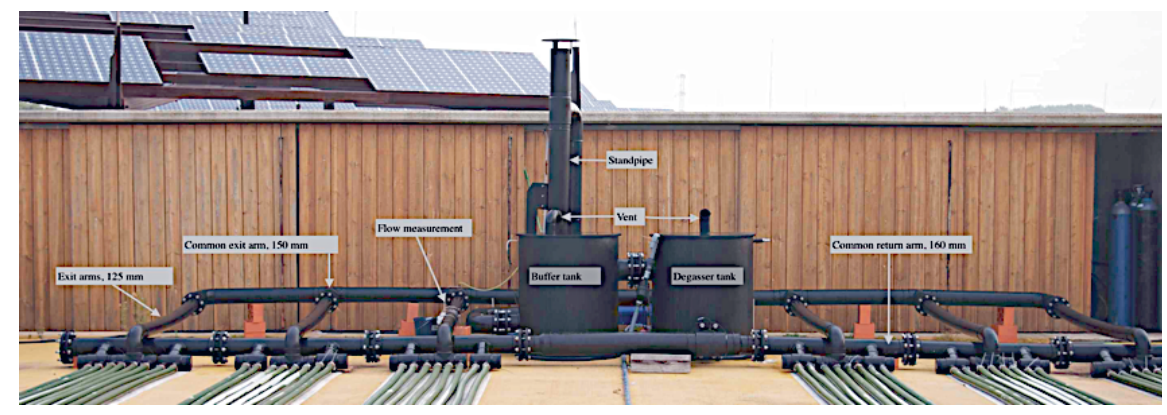

Fig. 6-3 The photobioreactor flow distribution system

manifold. Hard pipe dimensions in the distribution system varied from $200 \mathrm{~mm}$ to $125 \mathrm{~mm}$

(Fig. 6-3). The distribution system included a $1 \mathrm{~m}^{3}$ degassing tank with air-spargers and a $1 \mathrm{~m}^{3}$ buffer tank before the circulation pump. Before the exit main manifold branch, an open, 3-meter stand-tube was inserted to avoid the risk of over-pressuring the soft PE tubes. The total volume of the system was $6.1 \mathrm{~m}^{3}$ and the horizontally projected tube area was $92 \mathrm{~m}^{2}$. Degassing was carried out by air sparging.

The circulation pump was a KSB kwp K 100-250 with a $224 \mathrm{~mm}$ impeller with a design speed of $950 \mathrm{rpm}$ (low shear). The pump was controlled with a frequency converter according to pump speed or power consumption.

Dissolved oxygen was measured at two points - at the entrance to the degassing tank and before the circulation pump. 
$\mathrm{pH}$ was measured between the buffer tank and the circulation pump.

With a horizontal tubular reactor, harvesting represents a problem, particularly when used in batch mode. In this case, the reactor was emptied by gravity by manually lifting the tubes. Dimensions of the large tubular plant are given in Table 6-3.

Table 6-3 Characteristics of the large tubular plant.

\begin{tabular}{|l|l|}
\hline Photobioreactor dimensions and main operational parameters. & Dimensions \\
\hline Length of tubes & $32 \mathrm{~m}$ \\
\hline Tube length between degassing & $64 \mathrm{~m}$ \\
\hline Total projected area of tubing & $92 \mathrm{~m}^{2}$ \\
\hline Total volume of plant & $6.1 \mathrm{~m}^{3}$ \\
\hline Tube diameter & $6 \mathrm{~cm}$ \\
\hline Tubing material & $\mathrm{PE}, \mathrm{UV} \mathrm{stabilized}$ \\
\hline Tubing wall thickness & $150 \mu \mathrm{m}$ \\
\hline Tube pressure bursting point & $0.8 \mathrm{bar}^{-}$ \\
\hline Tube maximum sustained pressure & $0.2 \mathrm{bar}^{-1}$ \\
\hline Circulation pump flow max at 2.2 kW power supply & $900 \mathrm{~m}^{3} \mathrm{~h}^{-1}, 0.4 \mathrm{bar}$ \\
\hline Air flow (at a point just before pump) & $4 \mathrm{~m}^{3} \mathrm{hr}^{-1}$ \\
\hline Normal flow velocity (at 180 W power supply) & $0.45 \mathrm{~m} \mathrm{~s}^{-1}$ \\
\hline
\end{tabular}

\section{Operation of photobioreactor}

The plant was not hermetically closed; sparging air was unfiltered and the medium was made from 5- $\mu \mathrm{m}$ filtered seawater (made from tap water and natural sea-salt). At harvest, the tubes were emptied manually into the buffer tank by lifting the tubes and the culture broth was pumped to a harvest buffer tank, centrifuged and stored frozen at $-20 \mathrm{deg}$. As the centrifuge could process between 300 and $600 \mathrm{~L} \mathrm{hr}^{-1}$, depending on algal buoyancy, a harvest procedure, lasting 12-24 hours was implied from the point the circulation was turned off till the biomass was frozen.

Between batches, the plant was cleaned with a pressure cleaner, the tubing was renewed, and the plant sanitized with 200 ppm sodium hypochlorite, connecting the manifold studs with short sections of tubing.

\section{Temperature control}

Due to the late time of year (October/November), temperature control was not necessary as a maximum permissible temperature of 35 degrees was found for Neochloris and the daily temperature maximum only shortly reached that level on the day with the highest 
irradiation. During summer, temperature was controlled with high pressure water mist spray-nozzles positioned over the tube lanes with 2-meter intervals.

pH control

$\mathrm{pH}$ was controlled at 8.0 with $\mathrm{CO}_{2}$, injected before the circulation pump to ensure rapid mass transfer of gaseous to dissolved $\mathrm{CO}_{2} . \mathrm{pH}$ and dissolved oxygen were measured with a Prosense Qis P915-18 controller with recording of dissolved oxygen, $\mathrm{pH}$ and temperature data.

Flow velocity

Flow velocity was measured in one of the 6 outgoing 125-mm exit arms (Fig. 6-3) with a Sierra model 210 acoustic flowmeter. Flow velocity was set to about $0.45 \mathrm{~m} \mathrm{sec}^{-1}$ by adjusting the circulation pump frequency controller. The velocity measurement only worked with growth medium, presumably because small gas bubbles accumulate in the medium after algal growth takes on. As a consequence, the frequency controller settings were left constant during the cultivation period. The velocity may could therefore have changed during the day as a consequence of foam formation and gas pockets and over the cultivation period as a consequence of biofilm build up.

\section{Biofilm control}

Nylon beads (PA 10 with a positive buoyancy in sea water) were used during the batch. In the $600 \mathrm{~L}$ system, the beads passed perfectly unharmed through the circulation pump; in the $6 \mathrm{~m}^{3}$ reactor system, on the other hand, the beads were gradually macerated during the cultivation cycle. It is uncertain what effect - if any - the beads may have had on the biofilm formation.

\section{Oxygen control.}

Dissolved oxygen content was measured at two points in the plant: before the degassing tank and between the buffer tank and circulation pump (Fig. 6-3). The degassing tank was sparged with atmospheric air from two $60 \mathrm{~W}$ diaphragm pumps through 4 sintered, $12 * \varnothing 5$ $\mathrm{cm}$ diffusor cylinders, delivering $4 \mathrm{~m}^{3}$ of air $\mathrm{hr}^{-1}$. 
Foam and gas pockets.

10 ppm of antifoam Struktol 304 was added manually on demand, normally once per day, to prevent foam formation. Prior to usage in the reactor, antifoam dosage was independently assessed in the laboratory in order to find a suitable concentration which does not compromise algal growth (data not shown). Gas pockets were found to be able to accumulate at high points in the tubes as a result of the unleveled ground, and it was difficult to evacuate by raising the circulation pump velocity. Instead, gas pockets were removed manually on a daily base with a roller tool that was devised to drive the gas pockets out of the tubes.

\section{Harvest}

With a horizontal tubular reactor, harvest of the total batch is laborious; in this case, the polyethylene tubes were cut at the tube inlet one by one and lifted manually, walking towards the opposite manifold to evacuate the broth into the remaining reactor by gravity. During this procedure, the broth was pumped from the reactor to a harvest buffer tank from which it was pumped to a GEA KA6 semicontinuous bowl centrifuge. Biomass density obtained with this centrifuge was $21 \% \mathrm{dw}$.

\section{Results and discussion}

\section{Inoculation of the outdoor reactors.}

About $50 \mathrm{~g} \mathrm{dw}$ of biomass was used to start the $600 \mathrm{~L}$ reactor, resulting in an initial biomass concentration of $0.1 \mathrm{~kg} \mathrm{dw} \mathrm{m}^{-3}$, which is less than the $0.3 \mathrm{~kg} \mathrm{dw} \mathrm{m}^{-3}$, which was estimated necessary to start the reactor under full sunlight exposure (Chapter 5). Furthermore, the biomass had been kept dark at 4 degrees for a period of 2 weeks before use. The 600 -liter reactor tubes therefore initially were covered with white plastic which reduced incident irradiation about $80 \%$. The cover was removed in 3 steps between day 2 and day 3 . 
Fig. 6-4 shows that the growth of $N$.

oleoabundans in the $600 \mathrm{~L}$ reactor followed an exponential rate of 0.4 day $^{-1}$ without lag phase, suggesting that the irradiation exposure procedure was not excessive but also not light limited.

For the $6 \mathrm{~m}^{3}$ reactor, the entire broth from the $600 \mathrm{~L}$ culture was used as a starter culture.

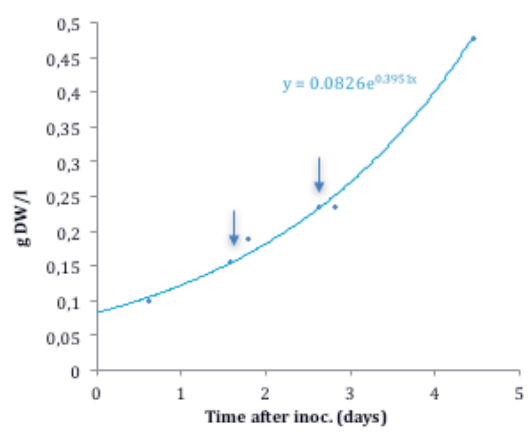
Inoculation was done in the evening; a

Fig. 6-4 Growth of Neochloris in the $600 \mathrm{~L}$ tubular photobioreactor. Arrows indicate time at which precaution to minimize the risk of first, respectively last $1 / 3$ of cover over reactor was photoinhibition in the beginning of the removed cultivation cycle and the resulting biomass concentration was $0.141 \mathrm{~kg} \mathrm{~m}^{-3}$ (Fig. 6-5).

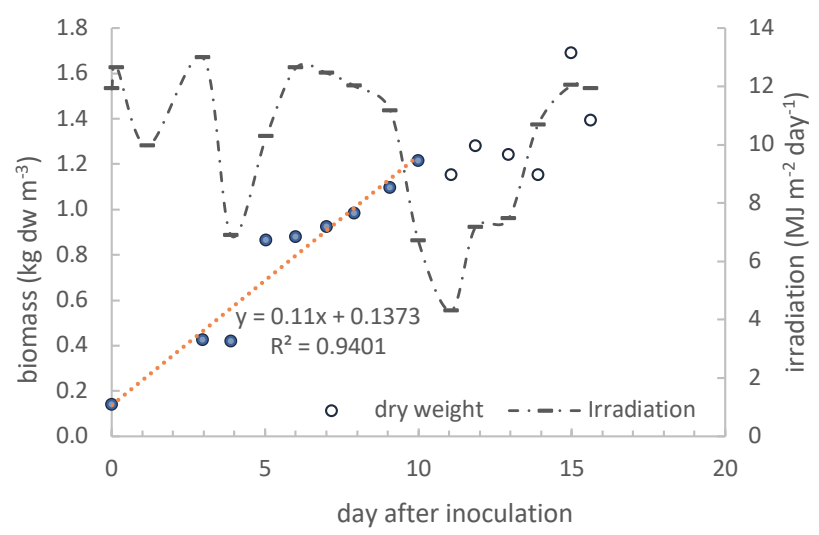

Fig. 6-5 Biomass concentration and irradiation in the $6 \mathrm{~m} 3$ reactor
A minimum of inoculum concentration of $0.3 \mathrm{~kg} \mathrm{~m}^{-3}$ had been predicted necessary. Biomass growth (Fig. 6-5) appeared to be approximately linear over the first 10 days of the batch cycle with no apparent sign of photo inhibition in the beginning. It is possible that the inoculum had been more

properly adapted to the outdoor conditions and therefore could sustain a higher irradiation or stronger dilution than the laboratory culture used to derive the minimum inoculum density.

\section{Fatty acid control}

For Neochloris, the relation between fatty acid content and inverse nitrogen ratio could be described according to the formula: 
$y=0.4807 * x^{2}+0.0893 * x+0.1107 \quad R^{2}=0.7$

(Equation 6-6)

with $y$ being total fatty acid content in biomass (TFA\% $\mathrm{dw}$ ) and $x$ being inverse nitrogen quota (g dw mmol-1 $\mathrm{N}$ ) (Chapter 5).

A biomass concentration of $2.73 \mathrm{~kg} \mathrm{dw} \mathrm{m}^{-3}$ and a total fatty acid content of $30 \% \mathrm{dw}$ was set as a target for the production.

Using the formula, the resulting target inverse nitrogen ratio of $0.54 \mathrm{~g} \mathrm{dw} \mathrm{mmol}^{-1} \mathrm{~N}$ for Neochloris for a total nitrogen addition of $31.05 \mathrm{~mol}$ would require a final biomass concentration of $2.73 \mathrm{~kg} \mathrm{dw} \mathrm{m}^{-3}$ (Table 6-4).

The nitrogen addition was taken as the total nitrogen used to produce the batch, including nitrogen added in the propagation step of the biomass as the biomass of the propagation reactor and the $600 \mathrm{~L}$ reactor was transferred as a total. Biomass concentration at harvest, however, reached only $1.4 \mathrm{~kg} \mathrm{dw} \mathrm{m}^{-3}$, down from 1.7 on the previous day due to cloudy conditions.

\section{Biomass productivity}

During the linear, nutrient replete growth phase from day 0 to day 10 where the residual nitrate concentration was exhausted, volumetric biomass productivity was $0.11 \mathrm{~kg} \mathrm{dw} \mathrm{m}$ day $^{-1}$ (the slope of the linear biomass trend in Fig. 6-5). This corresponds to an average areal biomass productivity of $7.3 * 10^{-3} \mathrm{~kg} \mathrm{dw} \mathrm{m}^{-2} \mathrm{day}^{-1}$ and a photosynthetic efficiency of $1.42 \%$ (Table 6-4). This is a rather low figure; with other algae, much higher values have been experienced in the present photobioreactor, for example with Chlorococcum littorale under identical conditions, a photosynthetic efficiency of $5 \%$ was obtained (data not shown).

An average of $1.5 \%$, was obtained with Nannochloropsis $s p$. in a horizontal tubular reactor in the Netherlands during July and August whereas the vertically stacked tubular photobioreactor yielded $2.4 \%$ on average (Vree et al. 2015).

But in nutrient replete, laboratory turbidostat cultures of Neochloris oleoabundans, a biomass yield on light of $0.99 \mathrm{~g} \mathrm{dw} \mathrm{mol}^{-1}$ photons was obtained for low light cultures (average intensity $70 \mu \mathrm{mol}$ photons $\mathrm{m}^{-2} \mathrm{sec}^{-1}$ ) and $0.76 \mathrm{~g} \mathrm{dw} \mathrm{mol}^{-1}$ photons for high light cultures average intensity $200 \mu \mathrm{mol}$ photons $\mathrm{m}^{-2} \mathrm{sec}^{-1}$ ) (Klok et al. 2013). This corresponds 
to a photosynthetic efficiency of 3.8 and $3.16 \%$ respective, using equation $6-5$. With increasing nitrogen deficiency, the biomass yield on light decreased strongly. Whereas it is clear that nutrient replete Neochloris may exhibit high photosynthetic efficiency, it is possible that both oleaginous species integrate the strong light gradients in the horizontal tubular reactor poorly, resulting in reduced photosynthetic efficiency.

\section{Harvest efficiency}

At harvest, which was carried out in a bowl centrifuge) only $52.5 \%$ of the biomass that the daily sampling had documented in the reactors, was recovered (Table 6-4). It is unclear what caused the biomass loss during harvest; the centrate appeared visibly clear, but the procedure was lengthy ( 8 hours) during which time the culture was stagnant. Fermentation in the algal biomass or loss of dissolved substances from excretion or cell disruption may have played a role.

\section{Lipid and fatty acid productivity}

Average volumetric lipid productivity during the nitrogen limitation period from day 10 to day 16 was $42 * 10^{-3} \mathrm{~kg} \mathrm{~m}^{-3}$ day $^{-1}$ and average areal productivity was $2.0 * 10^{-3} \mathrm{~kg} \mathrm{~m}^{-2}$ day $^{-1}$. Maximum lipid productivities that occurred over the last 2 days ( 3 datapoints) before the culture was harvested, were $149 * 10^{-3} \mathrm{~kg} \mathrm{~m}^{-3}$ day $^{-1}$ and $7 * 10^{-3} \mathrm{~kg} \mathrm{~m}^{-2} \mathrm{day}^{-1}$, respectively (Table 6-4).

The lipid concentration in the Neochloris culture reached $39 \%$ dw and TFA reached $14 \%$ $\mathrm{dw}$ at harvest while the predicted TFA was $17 \% \mathrm{dw}$ (Fig. 6-6).

Whilst close to the predicted value, it was far from the original planned content ( $30 \% \mathrm{dw}$ ). Main reason for this is that the biomass concentration only reached $1.40 \mathrm{~kg} \mathrm{dw} \mathrm{m}^{-3}$; significantly lower than the planned concentration of $2.73 \mathrm{~kg} \mathrm{~m}^{-3}$ (Fig. 6-5 and Table 6-4).

Having continued the cultivation of batch till it had reached a higher biomass concentration would have been the general obvious action to reach a higher inverse nitrogen quota - in the actual situation however, it is unlikely that it would have been effective as the weather remained dark (irradiation $6 \mathrm{MJ} \mathrm{m}^{-2}$ day $^{-1}$ ) for several days after the harvest which is approximately half the normal daily irradiation for the period (Fig. 6-5). 


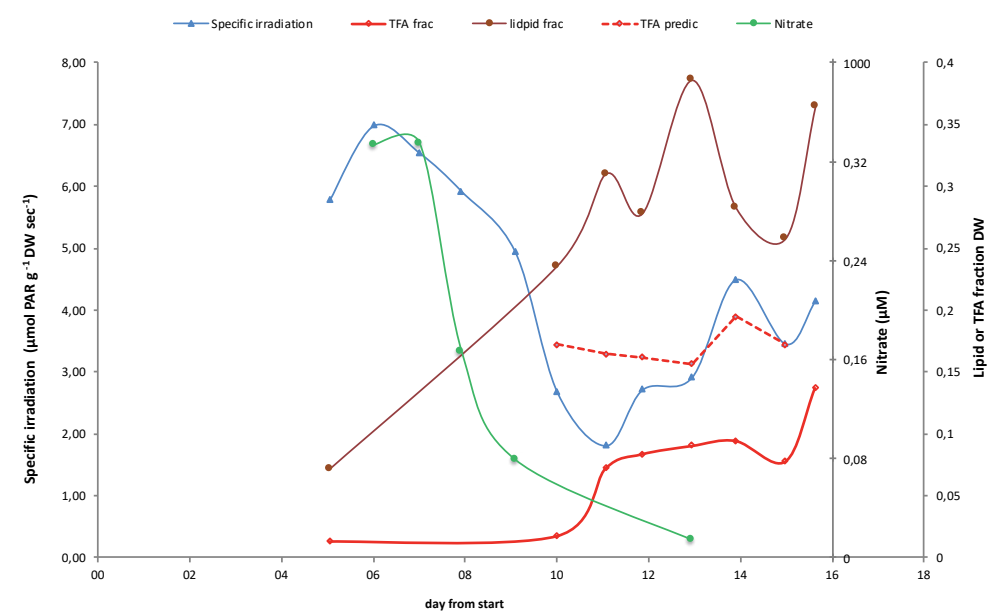

Fig. 6-6 Lipid fraction of biomass, TFA fraction of biomass, predicted TFA, specific irradiation and nitrate concentration under nitrogen limited Neochloris in the $6 \mathrm{~m}^{3}$ tubular reactor from 22.10.2011 to 07.11.2011. TFA was predicted according to the inverse nitrogen ratio method

Another way to raise the TFA content could have been to reduce the biomass density in the reactor as the biomass specific irradiation appears to be critical to obtain a high TFA yield. Janssen et al. found a maximum TAG yield on light between 11 and $26 \mu \mathrm{mol}$ photons $\mathrm{sec}^{-1} \mathrm{~g}$ $\mathrm{dw}^{-1}$ (Janssen 2018). Average (24 h) biomass specific irradiation for Neochloris is shown in Fig. 6-6 and it appears to be correlated to TFA and lipid concentration after $\mathrm{NO}_{3}$ is depleted. TFA appears to increase at a biomass specific irradiation above $4 \mu \mathrm{mol}$ photons $\sec ^{-1} g^{-1}$.

The proposed inverse nitrogen ratio method is thus a tool to help steer the fatty acid content in the biomass. It may help balancing the fatty acid production either towards high content with low biomass yield or low content with high biomass yield.

The accuracy of the inverse nitrogen quota method to predict the microalgal TFA content is not yet clear; Fig. 6-6 shows a discrepancy between the predicted and the realized fatty acid concentration.

The discrepancy may express a fundamental difference between the continuous light conditions under which Equation 6-6 is derived and the natural day-light cycle under which the outdoor cultivation is done. Another source of variability could be the sampling point of 
the day, if the TFA content in the biomass is very dynamic but further work is necessary to elucidate that.

Table 6-4 Characteristics of the Neochloris batch, produced 22.10.2011-07.11.2011 in Matalascañas, south-west Spain. Note that the enthalpy value of $21 \mathrm{~kJ} \mathrm{~g}^{-1}$ which was used in the photosynthetic efficiency calculation was assumed and not measured.

\begin{tabular}{|c|c|c|}
\hline Parameter & Unit & Value \\
\hline Nitrogen limitation & & yes \\
\hline Date of inoculation & & 22.10.2011 \\
\hline Date of harvest & & 07.11 .2011 \\
\hline Wet weight harvested & $\mathrm{kg}$ & 20 \\
\hline Dry weight harvested & $\mathrm{kg}$ & 4.49 \\
\hline Dry weight produced & $\mathrm{kg}$ & 8.6 \\
\hline Volumetric biomass productivity ( $\mathrm{N}$ replete) & $\mathrm{kg} \mathrm{m}^{-3}$ day $^{-1}$ & 0.11 \\
\hline Areal biomass productivity ( $\mathrm{N}$ replete) & $\mathrm{kg} \mathrm{m}^{-2}$ day $^{-1}$ & $7.3 * 10^{-3}$ \\
\hline Average irradiation in batch period & MJ m-2 day ${ }^{-1}$ & 9.9 \\
\hline Average irradiation lin. growth period & MJ m-2 day ${ }^{-1}$ & 10.6 \\
\hline Photosynthetic efficiency & $\%$ & 1.42 \\
\hline Average volumetric $\mathrm{N}$-deplete lipid productivity (day $10-16$ ) & $\mathrm{kg} \mathrm{m}^{-3}$ day $^{-1}$ & $41.9 * 10^{-3}$ \\
\hline Average areal N-deplete lipid productivity (day 10-16) & $\mathrm{kg} \mathrm{m}^{-2}$ day $^{-1}$ & $2.0 * 10^{-3}$ \\
\hline Maximum volumetric lipid productivity (day 14-16) & $\mathrm{kg} \mathrm{m}^{-1} \mathrm{day}^{-1}$ & $149 * 10^{-3}$ \\
\hline Maximum areal lipid productivity, (day 14-16) & $\mathrm{kg} \mathrm{m}^{-2}$ day $^{-1}$ & $7.0 * 10^{-3}$ \\
\hline Total period average lipid productivity (day 15) & $\mathrm{kg} \mathrm{m}^{-3}$ day $^{-1}$ & $32 * 10^{-3}$ \\
\hline TFA concentration max & $\% d w$ & 14 \\
\hline TFA concentration max, predicted & $\% \mathrm{dw}$ & 17 \\
\hline Nitrogen addition to reactor & $\mathrm{mol}$ & 31.1 \\
\hline Target Inverse nitrogen cell quota & $\mathrm{g} \mathrm{mmol}^{-1}$ & 0.54 \\
\hline Actual final inverse nitrogen cell quota & $\mathrm{g} \mathrm{mmol}^{-1}$ & 0.28 \\
\hline Biomass specific irradiation & $\mu \mathrm{mol} \mathrm{g}^{-1} \mathrm{sec}^{-1}$ & 4.37 \\
\hline Lipid productivity on light & mg lipid mol-1 & 120 \\
\hline Density (dw/ww) & $\%$ & 22.5 \\
\hline Recovery (biomass harvested/biomass produced) & $\%$ & 52.2 \\
\hline
\end{tabular}

\section{Power consumption}

Fig. 6-7 shows the volumetric power consumption of the circulation pump as a function of flow velocity. The recirculation energy applied for the duration of the batch was not directly measured, but for this and two following batches, a total of $1107 \mathrm{kWhr}$ were used by the circulation pump in the $6 \mathrm{~m}^{3}$ photobioreactor, corresponding to an average volumetric power supply of $166 \mathrm{~W} \mathrm{~m}^{-3}$. Average specific circulation power consumption was $28.7 \mathrm{kWhr}$ per kg of biomass produced. In a tubular pilot reactor facility, using $9 \mathrm{~cm}$ diameter tubing with a velocity of $0.9 \mathrm{~m} \mathrm{~s}^{-1}$, a volumetric power supply of $333 \mathrm{~W} \mathrm{~m}^{-3}$ resulted (Acién et al. 2012) but due to the different tube dimension and velocity, the two plants cannot be directly compared. 


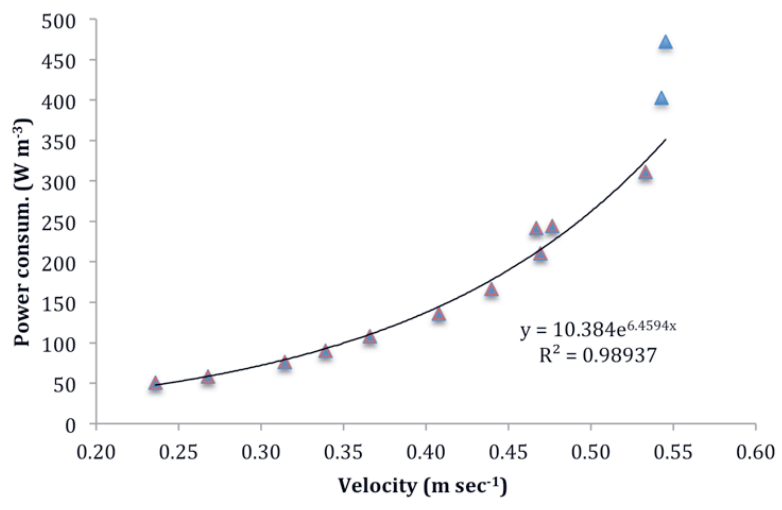

Fig. 6-7 Volumetric power consumption (based on tube volume) versus flow velocity in the $6 \mathrm{~m}^{3}$ tubular reactor on growth medium.

Note: the points in blue signature are not included in the regression
We calculated for a full scale (100

ha) tubular plant of a this design a circulation power supply of 76-133

$\mathrm{W} \mathrm{m}^{-3}$, depending on tube hydraulic roughness. The flow velocity used was $0.5 \mathrm{~m} \mathrm{sec}^{-1}$ (Norsker et al. 2012). In the pilot test, the velocity however was less than $0.5 \mathrm{~m} \mathrm{sec}^{-1}$. Unfortunately, the velocity could not be measured with algal culture in the tube, only with the sea water

medium, probably because bubble formation disturbed the sonic measurement.

With seawater medium in the current plant, the volumetric power consumption as a function of tube flow velocity is shown in Fig. 6-7. The regression line equation was $y=$ $10.384 \mathrm{e}^{6.4594 \mathrm{x}}, y$ being volumetric power consumption $\left(\mathrm{W} \mathrm{m}^{-3}\right)$ and $x$ being tube flow velocity $\left(\mathrm{m} \mathrm{sec}^{-1}\right)$. A flow velocity of $0.5 \mathrm{~m} \mathrm{sec}^{-1}$ would thus require $262 \mathrm{~W} \mathrm{~m}^{-3}$ that probably should be compared to the theoretical smooth-tube value of $76 \mathrm{w} \mathrm{m}^{-3}$, although patches of biofilm had formed in the tubes during the measurement period.

\section{Summary and conclusion}

The soft sleeve horizontal tubular reactor described here was constructed at relatively low costs. Strategies for culture propagation, inoculation of outdoor reactors, $\mathrm{pH}$ and oxygen control worked well during the cultivation period. Temperature control was not necessary due to the late time of the year. During summer periods, temperature control had been accomplished with mist spraying.

The horizontal soft-sleeve tubes used were generally practical for the microalgal production with a few critical points: The horizontal tubes accumulate gas pockets on the high spots if the ground is not sufficiently levelled. Foam or gas pockets must be removed to maintain light absorption and flow rate. This can in principle be achieved by increasing the circulation pump but there is a limit to how much pressure the tubing can withstand 
(burst pressure of the used polyethylene sleeves was 0.8 bar, but a 0.25 bar stand-pipe was included to prevent tube bursting and this effectively prevented proper evacuation of tubing using the circulation pump. Instead, a roller device was used to drive air-pockets out. Secondly, emptying the plant for harvest without destroying the tubing is also not possible and the tubing was therefore used as disposable item.

The inverse nitrogen quota is promising method to predict and control TFA concentration under nitrogen depletion in outdoor, tubular reactors; focus however should be on elucidating the influence of biomass specific irradiation on biomass productivity and TFA content. It may be inferred from the data that a biomass-specific irradiation of at least 4 $\mu \mathrm{mol}$ photons $\mathrm{g}^{-1} \mathrm{sec}^{-1}$ is a perquisite for good biomass growth and hence TFA formation under nitrogen limitation. 


\section{Generall discussion}




\section{Achievements of the thesis work}

\section{Chapter 2}

The productivity and economics in photobioreactor production was analyzed. Typical photosynthetic efficiency was found to be around $1.5 \%$ in open ponds, $3 \%$ in horizontal tubular reactors and $5 \%$ in flat panel reactors was identified, based on the rather limited available literature at the time (Norsker et al. 2011). With these data and Dutch irradiation data, annual biomass productivity in the Netherlands amounted to 21 -, 41- and 64-ton dw $\mathrm{ha}^{-1}$ year $^{-1}$ and biomass production costs $4.95,5.15$ and $5.96 € \mathrm{~kg}^{-1}$ for a 100 -ha plant. The paper has received 700 citations (Google Scholar) and still has a high citation rate despite being almost 10 years old. This paper gave insight in the techno-economic bottlenecks in the production processes for microalgae and helps to fine-tune research questions to address in further technological developments of the process.

In 2016, the techno-economic model was updated (Ruiz et al. 2016), a more detailed design supported by 4 years of empirical experience from pilot plants in AlgaePARC, Wageningen and extrapolations were made towards locations with more sunny conditions. In addition, the effect of scale was studied. Photosynthetic efficiency established was 2.4 and $2.7 \%$ for vertical tubular reactors and flat panels, respectively. Presently predictions show that costs can be as low as $3.4 € \mathrm{~kg}^{-1}$ if production takes place in southern Spain. Sensitivity analysis showed that cost reduction toward $0.5 € \mathrm{~kg}^{-1}$ are possible with as major factors the photosynthetic efficiency and scale of production. Evaluating the photosynthetic efficiency predictions that are the basis for the projections, the figure for the tubular plant (3\%) in the Netherlands was close to the empirically established figure for the tubular plant in AlgaePARC (2.4\%). It should be kept in mind, however that the tubular plant in chapter 2 was a horizontally extended, whereas the tubular plant described in Ruiz et al. (2016), was vertically stacked which would expectedly exhibit a somewhat higher photosynthetic efficiency. The estimated photosynthetic efficiency of the vertical panel reactor in chapter 2 was $5 \%$ against an empirical result of $2.7 \%$. This is a considerable discrepancy which presumably is due to the small literature value base, the prediction in chapter 2 was based upon. Biomass production costs (100 ha scale) were in chapter 2 
estimated to 5.15 and $5.96 € \mathrm{~kg}^{-1}$ for a horizontal tubular and flat panel plant respective against 8.9 and $6.0 € \mathrm{~kg}^{-1}$ in the experimentally supported projection. With the flat panel plant, emprical photosynthetic efficiency was less than anticipated in chapter 2 , but the panel height was $0.5 \mathrm{~m}$ as opposed to $1 \mathrm{~m}$ in chapter 2 , and aeration costs presumably therefore smaller. In the study of Ruiz et al. (2016), the flat panel resulted as the most cost-efficient with about $30 \%$ lower biomass production costs. Sensitivity analyses in the two studies did not show any major differences. Based on this assessment, research questions 1 . can be said to have been adequately answered.

\section{Chapter 3}

In the years 2010-2011, specific energy requirements for pumping microalgae in tubular reactors from 2000-4000 $\mathrm{W} \mathrm{m}^{-3}$ were frequently cited and re-cited; one paper even indicated $6000 \mathrm{~W} \mathrm{~m}^{-3}$. Energy costs for pumping were so high that we decided to analyze the calculations in depth. We showed that it is possible to reduce the energy input close to $100 \mathrm{~W} \mathrm{~m}^{-3}$ (Norsker et al. 2012). In the 1990'es, there was a concern with cell damage caused by shear stress in conventional centrifugal pumps and as a precautionary measure, pumping widely done with air-lift pumps that in most photobioreactor installations require a large power supply. This may for a while have delayed the development of tubular photobioreactors. To-day, centrifugal pumps with energy requirements of $100-300 \mathrm{~W} \mathrm{~m}^{-3}$ are used with most tubular photobioreactors.

Another design modification, foreseen in the paper, regarded the height of flat panel reactors, that in some experimental designs from the time were about one meter or higher. A height of $0.5 \mathrm{~m}$ was predicted as energetically maximum feasible because low panel height reduces the air pressure required to sparge the panels. This means that blowers may be used instead of compressors with significant reductions both in operating and capital costs and low height panels $(0.5 \mathrm{~m})$ are now applied in for instance a commercially produced flat panel reactor system. Calculations furthermore indicated that a positive net-energy balance could be achieved for flat panel reactors at a Dutch site at an aeration rate less than $0.25 \mathrm{~V} / \mathrm{V}$ (volumes of gas delivered per panel volume). At this point, the reactor would start to produce more energy by photosynthesis than it consumed for circulation. For tubular reactors, this would occur at flow velocity under $0.35 \mathrm{~m} \mathrm{sec}^{-1}$ 
(Norsker et al. 2012). Based on personally given information by a tubular plant supplier, specific pumping energy supply may be under $100 \mathrm{~W} \mathrm{~m}^{-3}$. The height of commercially produced flat panel reactors are now 0.5 . These facts suggest that the treatment of research question 2 was adequate.

\section{Chapter 4}

The chapter is an industrial scale validation of productivity model of a flat panel photobioreactor with panels, suspended by buoyancy in a waterfilled plastic bag. The design of the reactor was done by a Belgian chemical company inspired by the flat panel reactor in chapter 2 although not entirely in that it applied closely spaced panels to obtain mutual shading and reflection between the panels. The purposes for following-up the development with a productivity model were 2 :

a) develop a tool to help optimizing the design and operation of the photobioreactor

b) provide a benchmark for performance evaluation of the reactor.

The model is relatively complex because actual irradiation and temperature data was used as input at a given site for instantaneous productivity simulation with a reactor with a complex geometry. The irradiation absorbed by the reactor panels was calculated with a ray tracing model that calculates vertical distribution of irradiation on the panels. This requires information about the direct/diffuse light proportion and as such information normally is not readily available, the proportion was estimated from atmospheric clearness formulae. Average light intensity inside the panel at a given height was then calculated, followed by instantaneous growth rate model (temperature and light intensity based) for the given strain (Fig. 7-

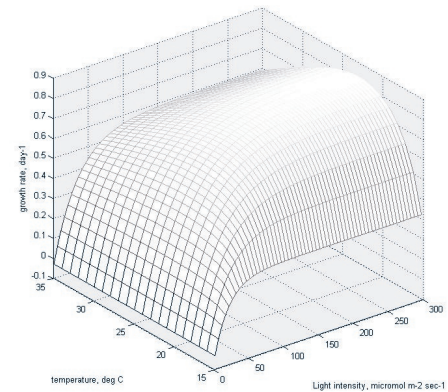

Fig. 7-1 Growth rate of Nannochloropsis as function of temperature and light intensity.

1) and from that, the instantaneous productivity rate was calculated, and other performance parameters, such as photosynthetic efficiency. To obtain a high temporal resolution empirical parameter, used for validation of the model, carbon dioxide absorption measurements were used to calculate the biomass productivity rate. Validation 
was carried out with a 20-reactor installation in Antwerp, each reactor with 35 flat panels and showed that the model predicted the empirical productivity rate well, both for a bluesky day and with rapidly changing cloud cover. On a heavily overcast winter day with negative 24-hour productivity on the other hand, the modelled day time photosynthetic efficiency was higher than measured because there is no independent respiration term. The model is of value because it demonstrates productivities can be simulated under dynamic conditions based on only 2 variables, that are easy to measure, horizontal irradiation and temperature.

Using the model on new scenarios with same strain and reactor is very simple and could be extended with raytracing models of other reactor designs and growth models for other algal strains could be included. This could be turned into a versatile photobioreactor simulation tool. The work developing the growth rate model applied in this work was substantial in that long term turbidostat steady state cultivations were carried out with only one laboratory flat plate reactor. To-day, there are several multiple reactor laboratory systems commercially available and data can be produced much faster. The model was furthermore used in the development of the photobioreactor to show effects of distance between panels, reflectivity and transparency of materials used and orientation and geographical location of reactor.

The model predicitons and the validation approach demonstrated that the research question 3 was successfully answered.

\section{Chapter 5 and 6}

Chapter 5 and 6 are full-scale system validation studies made with a horizontal tubular reactor with disposable, soft polyethylene tubes, as described in work in chapter 2. Development of the procedures is described in Chapter 5 and the validation for Neochloris oleoabundans in Chapter 6. 
The overall objective was to test whether oil-rich biomass, could be produced with the system on a high irradiation site. The reactor was designed by us for sequential batch operation and intermittent cleaning. There are many concerns in the design of a large scale microalgal production system, including dimensioning of a manifold system to ensure even flow in the 48 tubes, suitable degassing and temperature control and growth medium provision, a propagation chain that could provide a sufficient quantity of starter culture for the reactor. With smaller systems, the risk of irreversible photoinhibition and subsequent loss of the culture can be mitigated by covering the reactor the first few days after inoculation but with an industrial size reactor, this is not possible and the photoinhibition is instead avoided by starting the reactor with sufficient amount of culture. Minimum start biomass concentration is strains- and physiological state specific and required a scientific approach, given that the

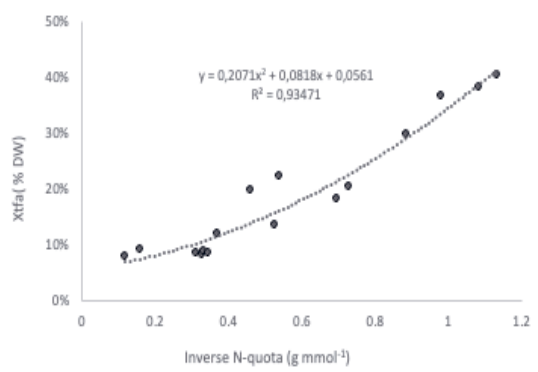

Fig. 7-2 The total fatty acid content (Xtfa) as function of the inverse nitrogen quota for Chlorococcum littorale.

alternative "having simply enough inoculum" is

not a practical one. Selecting a suitable strain for a new and unproven production purpose is also a challenge; test criteria should include both high oil content and high oil productivity but also amenability to the cultivation conditions and suitability for downstream processing. In the cultivation process, oil enhancement is also a consideration which is done by inorganic nutrient limitation and a procedure for controlling the nutrient limitation in the batch cultivation had to be developed. A Bligh-and-Dyer total fatty acid gas chromatograph analysis was adopted as the most suitable microalgal oil content analysis. At the point of planning the work, efforts had been done to develop fast lipid analysis procedures, but no practical approaches had resulted. Fast fatty acid results were required if they were to be used to control the cultivation process; normally, a GC analysis protocol is lengthy and organized as an automated, central service with long delays for the results. A proxy method to predict total fatty content and control the oil enhancement process was therefore developed (the inverse nitrogen ratio method, Fig. 7-2).

The validation did not produce as high fatty acid contents as planned - the nitrogen

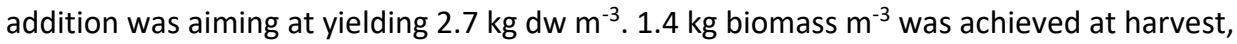


due to overcast sky the last several days of the cultivation period. With the biomass produced, $17 \%$ total fatty acid should have been found in the biomass as opposed to actual $13 \%$ total fatty acid content. It is difficult to know whether to conclude that the inverse nitrogen quota did or did not predict the fatty acid content so the research question 4 can therefore not be unambiguously answered.

The inverse nitrogen quota may nevertheless be a useful means to control fatty acid formation in industrial scale batch productions.

\section{The larger picture}

One may get the impression that microalgal $R \& D$ community has missed the outlook to an industrial main stream technology. The question is if this is true and justified or there is still a potential for such a breakthrough. The history of the field has not been an immediately success story, but a rather bumpy ride.

Microalgae have several times during the last 70 years attracted attention as promising candidates for industrial exploitation. In comparison with plants, microalgae have a rapid growth and have high potential for nutritional or therapeutic uses either as whole-cell biomass or as a biorefinery feedstock. But like the objectives "Staple food for the world population" and "Carbon dioxide emission mitigation", also "Biofuel after peak oil" lost industrial relevance necessary break-throughs could be made. In the "peak oil" days, where crude oil prices rose above $\$ 100$ per barrel, automotive fuels were the main driver behind the microalgal development. A few automotive and energy companies (eg. Isuzu, Mazda, Exxon-Mobile) still have microalgal programs. But most microalgal production companies have shifted towards food supplements and other niche products. Also, the Japanese company, Euglena that has large microalgal biofuel development programs, is currently exploring niche products.

But this does not mean that low value - large volume products with microalgae should be forgotten. As shown in chapters 5 and 6 , the potential to utilize the solar energy with an efficiency of up to $6 \%$ photosynthetic efficiency is there and molecular engineering may contribute to raise the photosynthetic efficiency, potentially above the $6 \%$ level. And the fact that the technological engineering has proven more difficult and slower than initially expected, should not discourage us from continue to investigate its opportunities. 
There are reasons for being optimistic about the microalgal development potential.

\section{Current microalgal production knowledge and know-how}

What is different now from previous algal "waves" as mentioned in Chapter 1 , is that there is now a much more solid knowledge base and an entrepreneurial layer, consisting of many smaller and larger players. There is a constantly increasing flow of scientific publications on the subject, currently about 500 publications per year were identified in a search in Science Direct (Fig. 7-3).

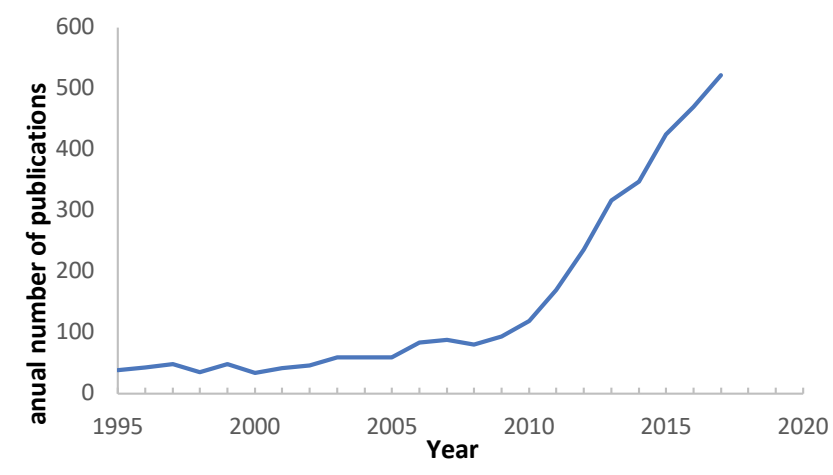

Fig. 7-3 The annual number of scientific publications in Science Direct, with the terms "alga" and "production" occurring anywhere. The publication types included Research papers, Reviews, mini-Reviews and Book Chapters. 55\% of the publications were in 4, predominantly applied journals: Bioresource Technology, Algal Research, Aquaculture and Journal of Biotechnology.
In Europe, currently 430 operating microalgal companies are known (Vieira 2017). 166 companies are aiming at commodity productions (Laurens et al. 2017). Companies are typically very small with $<10$ employees and have therefore difficulties funding R\&D. In the US, companies tend to be larger than in Europe, but still even large production facilities are smaller in extension than an average terrestrial crop producing farm and can therefore not finance serious process development efforts.

During the current wave of microalgal interest, we have experienced significant advances in the understanding of the physiological constraints in achieving high photosynthetic efficiency at high and variable irradiation but there are still significant practical engineering barriers to overcome. At AlgaePARC in Wageningen, photobioreactor designs are being developed and tested and systems are being evaluated under high irradiation sites such as southern Spain and Bonaire; the European union is supporting joint R\&D efforts and research groups and companies are working together in taking the production technology 
forward; this has resulted in commercially available flat panel- and tubular photobioreactor systems. But these systems are still costly and largely one-off installations and microalgal markets still focus on high-value products; unlocking the mutual constraints of high-volume market and production costs is therefore the key to future development.

At the moment, microalgal cultivation is experiencing rapid growth in China, including scale-up of photobioreactor technology (Fig. 7-4).

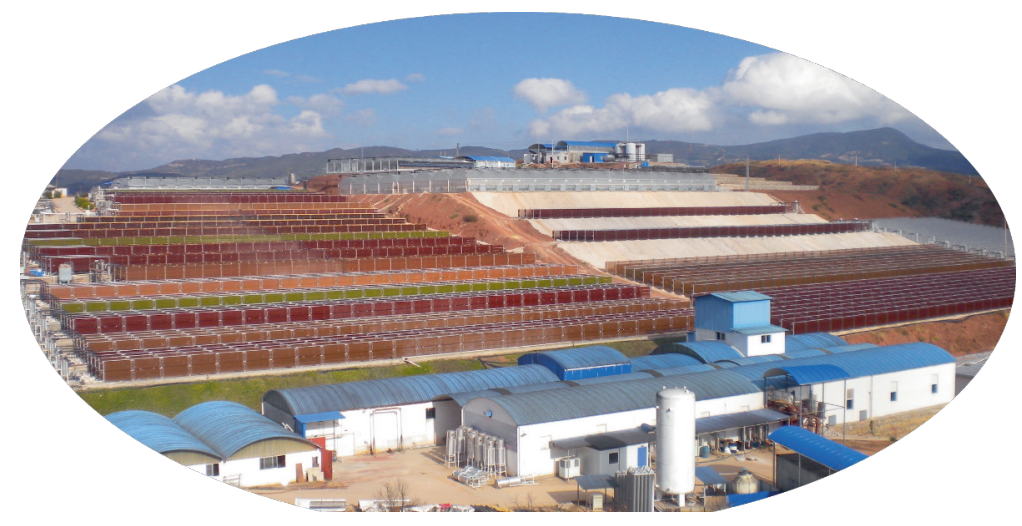

Fig. 7-4 Yunnan Alphy Biotech Co, a tubular photobioreactor plant, China (Haematococcus). Photograph: Courtesy of prof. Liu.

The practical aspects of microalgal photobioreactor development and management are not extensively treated in the scientific literature.

It is on one hand not typical academic practice - but also because technical and management aspects of professional microalgal production are kept secret not to reveal important know-how or business weaknesses. Better integration of academic science and entrepreneurship might help the area to move forward.

\section{Evaluating the photosynthetic efficiency of photobioreactors.}

In order to be able to judge reported production results and future development potentials, the photosynthetic efficiency of the process should be known and evaluated against the theoretically possible and benchmarked against that of successfully managed experimental reactors. This theoretical photosynthetic efficiency is established incontroversially as follows:

The first product that emerge from the photosynthesis, in which photons are captured and used to convert carbon from $\mathrm{CO}_{2}$ to organic matter, is glyceraldehyde 3-P or GAP. From 
GAP, glucose and acetyl-coenzyme-A, which is the building block for oils and carotenoids, originate.

The basic balance that has been standing since the 1950 'ies is, that to fix one carbon atom from $\mathrm{CO}_{2}, 8$ photons are required.

For glucose formation, the energy conservation in therefore is:

For red light, for example, energy (E) of a mol photons is $E=h * v * A=176 \mathrm{~kJ} \mathrm{~mol}^{-1}$; as $v=4.4110^{14} \mathrm{sec}^{-1}\left(\right.$ for $\lambda_{\text {red light }}=680 \mathrm{~nm}$ ), $h=6.63 * 10^{37}$ and $A=6.22 * 10^{23}$ The reason red light is chosen is that the additional energy of photons at shorter wavelengths is dissipated. Photons in the photosynthetically active spectrum, $400-700 \mathrm{~nm}$, yield the same energy as red light-photons.

Glucose consists of 6 carbon atoms, each requiring 8 photons to be fixed. Therefore, 48 mol photons are required for each mol glucose formed (Barber 2009) and the photosynthetic energy input is $48 \times 176 \mathrm{~kJ}$. Considering the energy in glucose as the standard enthalpy of combustion, $2805 \mathrm{~kJ} \mathrm{~mol}^{-1}$ (NIST 2017), the energy conservation of glucose formation on photosynthetically active light (PAR) is $2805 \mathrm{~kJ} /(48 \times 176 \mathrm{~kJ})=33 \%$. The photosynthetically active light represents on the average, however only about $42 \%$ of the energy in the full solar spectrum so the theoretical maximum energy conservation in glucose formation on daylight is about $33 \times .42=14 \%$.

Conversion to complex biomass however involves thermodynamical energy loss; there is photorespiratory loss involved in $\mathrm{CO}_{2}$ fixation and general biomass maintenance energy loss. Maximum theoretical daylight efficiency of microalgal biomass production is around 10\% (Tredici et al. 2010) or 9 \% (Janssen et al. 2003). In comparison, best photovoltaic technology reaches nearly $30 \%$ efficiency on daylight at the moment.

Empirical values from outdoor photobioreactors rarely exceed 4.5-6 \% (Barber 2009, Tredici et al. 2010, Work et al. 2012) and routinely often only around 3\%. Photosynthesis works at high efficiency only at rather low light intensity and it is a challenge to administer the often highly dynamic daylight to the culture so that it is used effectively. The closely spaced flat panel reactor (Chapter 4), however exhibited a carbon dioxide assimilation that responded well to a highly variable solar irradiation. 
In laboratory-, continuously illuminated photobioreactors, high productivities at high photosynthetic efficiencies at high irradiance are reported; the most impressive results in that sense was a dual-side illuminated, flat panel Spirulina culture $\left(2000 \mu \mathrm{m} \mathrm{m}^{-2} \mathrm{sec}^{-1}\right)$, exhibiting a productivity of $19 \mathrm{~g} \mathrm{dw} \mathrm{m}^{-2} \mathrm{hr}^{-1}$. Based on photosynthetic active radiation (PAR), efficiency was 15\% (Qiang et al. 1998). Equivalent solar efficiency is 6.3 A similar photosynthetic efficiency level was obtained with Chlorella sorokiniana in a vertical flatpanel photobioreactor, operated in luminostat mode under simulated summer southern Spanish daylight cycles: biomass yield on light was $1.3 \mathrm{~g} \mathrm{dw} \mathrm{mol}^{-1}$ photons as opposed to a theoretical maximum value of 1.8 (Cuaresma et al. 2011). Recalculated, assuming a theoretical maximum daylight photosynthetic efficiency of $9 \%$, it would correspond to a daylight photosynthetic efficiency of $6.5 \%$.

With outdoor photobioreactors, sofar lower photosynthetic efficiencies have resulted. At a Dutch photobioreactor test site, maximum photosynthetic efficiency was $4.2 \%$ with average $2.4 \%$ was found for July - December with a vertical tubular photobioreactor, maximum $3.8 \%$ for a flat panel reactor with average 2.7\% (Vree et al. 2015).

But high outdoor photobioreactor photosynthetic efficiency results do exist: Optimizing biomass concentration of Spirulina of outdoor flat panel reactors, PAR efficiency from 10 to $14 \%$ (daylight efficiency 4.3-6.02\%, recalculated) were obtained in June in Israel (Qiang et al. 1998), i.e., at irradiance reaching roughly similar levels as the irradiance as the above-mentioned laboratory flat panel experiments.

Maximum photosynthetic efficiency appears to be associated with intermediate biomass concentration levels (Molina Grima et al. 1996, Vree et al. 2015). A rather recent discovery is that maximum photosynthetic capacity and maximum TAG formation rate under nitrogen deficiency were shown to be associated with medium biomass-specific photon supply rates in Nannochloropsis gaditana (Janssen 2018). This may contribute to photobioreactor optimization and industrial algae production.

Another aspect of the photobioreactor development that has taken a toll on the optimism, is the auxiliary energy consumption of photobioreactors. But - as shown in Chapter 3 , some of the negative opinions and practices were based on wrong assumptions and auxiliary energy consumption of both tubular and flat panel reactors is now much less worrying. 


\section{A future for photobioreactors}

It is within reach to produce omega-3 fatty acids that have a large market (Chauton et al. 2015). And aviation could become a market for microalgal derived fuels. There is still a cost-gap for this market to be realistic but certainly realistic if photobioreactors are optimized to reach their already demonstrated potential.

Todays global scene is characterized by climate change mitigation, a massive green transformation of the industrial raw material supply, a trend towards electrical person transport vehicles, depending on sustainable energy and maybe power-to-methanol technology on the horizon. This development is not driven by raw competitive economy but environmental- and climate change motivation and microalgal fuels could be considered as well. The question how much the cost of microalgal fuels should be reduced to be realistic as an aviation fuel.

In the techno-economic analysis of Ruiz et al. (Ruiz et al. 2016), increasing photosynthetic efficiency from $2.7 \%$ to $6 \%$, biomass production costs could be reduced by $47 \%$ which would bring biomass production costs down to $€ 1.8 \mathrm{~kg}^{-1}$. As previously mentioned, a $6 \%$ photosynthetic efficiency in outdoor carefully managed experimental photobioreactors has been demonstrated, so such an improvement of the industrial practice is feasible. Capital costs, furthermore, constitute more than $30 \%$ of the biomass production costs of the biomass production for a 100-ha installation, even with the benefits of scale. As previously mentioned, current days photobioreactors are largely one-off installations, using central and costly off-the-shelf process equipment.

Cheaper, mass produced photobioreactors would be instrumental to increase the capacity and cost efficiency of the microalgae production. Economy-of-scale is difficult to realize in a short time span. Public-private partnerships in the field of photobioreactor development and manufacturing could lead to significant technology cost reductions and prepare for the green industrial conversion.

Engineering accomplishments normally are driven by market-motivation. We should not wait for the next wave of global economy-driven R\&D incentive but keep in mind, that industrial microalgal production is a complex biological practice and it requires strategically directed and sustained development efforts. 
As stated in Chapter 1, marine aquaculture development in Norway is a good example of a concerted, surprisingly fast private-public partnership development of a new biological industry.

Considering some of the near-feasible bulk-product opportunities, large concerted publicprivate engineering efforts could lead to cost-effective production technology in a rather short time. Given the stage of technology - 10 years could now result in a significant leap. 


\section{Completed training activities}

Category A: Discipline specific activities (courses, workshops, symposia, summer schools, conferences etc.)

\section{Name of the course/meeting}

8. Int conference on Applied Algology

9. Int Conference on Applied Phycology

Mar Biotechnol: Basic

14. Internat Symp on Carotenoids

International Algae Congress

Themamiddag Algenkweek

IROP Vergadering

EABA first conference

Management of Microbiolical Hazards in Foods

GOED Exchange

Category B: General courses (e.g. $\mathrm{PhD}$ week, writing and presenting courses, statistics, etc.)

Name of the course

Citation analysis for staff

GMP audit training course

Category C: Other activities (participation in

discussion groups, $\mathrm{PhD}$ excursions, MSc courses, etc.)

Name of the course

BioProcess A/S

Writing Research Proposal

\section{Organizing institute (s)}

Isapo

Isapo

BioProcess Engineering, WUR

International carotenoid society

DLG Benelux

Vereniging van Milieuprof

IROP

EABA

VLAG

GOED

\section{Organizing institute}

WUR library

Proviron Industries N.V.

\section{Organizing institute}

DK devel comp

BPE, WUR 


\section{Publication list}

Norsker, N.-H. (1991). Sulfide generation in biofilm in gravitary sewers. Vand og Miljø(Special Issue).

Norsker, N.-H. and J. G. Støttrup (1991). Technical and Biological Aspects of Continuous Micro algae Cultivation. European Aquaculture Society, Special Publication No. 15(15): 8790.

Nielsen, P. H., K. Raunkjaer, N. H. Norsker, N. A. Jensen and T. Hvitved-Jacobsen (1992). Transformation of waste-water in sewer systems - a review. , Pergamon-Elsevier Science Ltd.

Norsker, N. H. and J. G. Støttrup (1994). The importance of dietary HUFAs for fecundity and HUFA content in the harpacticoid, Tisbe holothuriae Humes. Aquaculture 125(1-2): 155166.

Støttrup, J. G. and N.-H. Norsker (1994). The importance of dietary HUFA's for fecundity and HUFA content in the Harpacticoid Tisbe holothuriae Humes. Aquaculture 125: 155-167.

Norsker, N.-H., P. H. Nielsen and T. Hvitved-Jakobsen (1995). Influence of oxygen on biofilm growth and potential sulfate reduction in gravity sewer biofilm. Water Science and Technology 31(7): 159-167.

Støttrup, J. G., K. Gravningen and N.-H. Norsker (1995). The role of different algae in the growth and survival of turbot larvae (Scopthalmus maximus L.) in intensive rearing systems. ICES mar. Sci.Symp. 201: 173-186.

Støttrup, J. G. and N. H. Norsker (1997). Production and use of copepods in marine fish larviculture. Aquaculture 155(1-4): 231-247.

Michels, M. H. A., A. J. van der Goot, N. H. Norsker and R. H. Wijffels (2010). Effects of shear stress on the microalgae Chaetoceros muelleri. Bioprocess and Biosystems Engineering 33(8): 921-927.

Norsker, N. H., M. Barbosa and R. Wijffels (2010). Microalgal Biotechnology in the Production of Nutraceuticals. Biotechnology in Functional Foods and Nutraceuticals. Debasis Bagchi, F. C. Lau and D. K. Ghosh, Taylor \& Francis: 591.

Norsker, N.-H., M. J. Barbosa, M. H. Vermuë and R. H. Wijffels (2011). Microalgal production - A close look at the economics. Biotechnology Advances 29: 24-27. 
Norsker, N.-H., M. J. Barbosa, M. H. Vermuë and R. H. Wijffels (2012). On Energy Balance and Production Costs in Tubular and Flat Panel Photobioreactors.

Technikfolgenabschätzung - Theorie und Praxis 21(1): 8.

Chauton, M. S., K. I. Reitan, N. H. Norsker, R. Tveterås and H. T. Kleivdal (2015). A technoeconomic analysis of industrial production of marine microalgae as a source of EPA and DHA-rich raw material for aquafeed: Research challenges and possibilities. Aquaculture 436: 95-103.

Norsker, N. H., M. Michiels, P. M. Slegers, G. L. A. M. Swinkels, M. J. Barbosa and R. H. Wijffels (2019). Productivity of Nannochloropsis oceanica in an industrial closely spaced flat panel photobioreactor. Algal Research 43: 101632.

Norsker, N.-H. (2020). Scale-up of microalgae-based processes. Handbook of MicroalgaeBased Processes and Products, Elsevier: 861-883. 


\section{Bibliography}

Aalborg-Portland (2018). Environmental Repoert 2018 kEnvironment and Helath \& Safety. Denmark, Alborg Portland A/S.

Acién Fernández, F., F. García Camacho, J. Sánchez Pérez, J. Fernández Sevilla and E. Molina Grima (1998). Modeling of Biomass Productivity in Tubular Photobioreactors for Microalgal Cultures: Effects of Dilution Rate, Tube Diameter, and Solar Irradiance. Biotechnology and Bioengineering 58(6): 605-616.

Acién Fernández, F. G., J. M. Fernández Sevilla, J. A. Sánchez Pérez, E. Molina Grima and Y. Chisti (2001). Airlift-driven external-loop tubular photobioreactors for outdoor production of microalgae: assessment of design and performance. Chemical Engineering Science 56(8): 2721-2732.

Acién Fernández, F. G., C. B. Alías, M. a. C. García-Malea López, J. M. Fernández Sevilla, M. a. J. Ibáñez González, R. Núñez Gómez and E. Molina Grima (2003). Assessment of the production of $13 \mathrm{C}$ labeled compounds from phototrophic microalgae at laboratory scale. Biomolecular Engineering 20(4): 149-162.

Acién, F. G., J. M. Fernández, J. J. Magán and E. Molina (2012). Production cost of a real microalgae production plant and strategies to reduce it. Biotechnology Advances 30(6): 1344-1353.

Adams, C., V. Godfrey, B. Wahlen, L. Seefeldt and B. Bugbee (2013). Understanding precision nitrogen stress to optimize the growth and lipid content tradeoff in oleaginous green microalgae. Bioresource Technology 131: 188-194.

Ahmad, F. B., Z. Zhang, W. O. Doherty and I. M. O'Hara (2018). Optimising Extraction Of Microalgal Oil Using Accelerated Solvent Extraction By Response Surface Methodology. Journal of Engineering Science and Technology 13(4): 964-976.

Alexander, P., C. Brown, A. Arneth, J. Finnigan and M. D. A. Rounsevell (2016). Human appropriation of land for food: The role of diet. Global Environmental Change 41: 88-98.

Alías, C. B., M. C. García-Malea, F. G. Acién, J. M. Fernandez, J. L. García and E. Molina (2004). Influence of power supply in the feasibility of Phaeodactylum tricornutum cultures. Biotechnology and bioengineering 87(6): 723-733.

Barber, J. (2009). Photosynthetic energy conversion: natural and artificial. J Chemical Society Reviews 38(1): 185-196. 
Barton, A. F., M. R. Wallis, J. E. Sargison, A. Buia and G. J. Walker (2008). Hydraulic roughness of biofouled pipes, biofilm character, and measured improvements from cleaning. Journal of Hydraulic Engineering-Asce 134(6): 852-857.

Béchet, Q., A. Shilton and B. Guieysse (2013). Modeling the effects of light and temperature on algae growth: state of the art and critical assessment for productivity prediction during outdoor cultivation. Biotechnology advances 31(8): 1648-1663.

Beer, L. L., E. S. Boyd, J. W. Peters and M. C. Posewitz (2009). Engineering algae for biohydrogen and biofuel production. Current Opinion in Biotechnology 20(3): 264-271.

Ben-Amotz, A. and M. Avron (1990). The biotechnology of cultivating the halotolerant alga Dunaliella. Trends in Biotechnology 8: 121-126.

Benemann, J. R. and W. J. Oswald (1996). Systems and economic analysis of microalgae ponds for conversion of $\mathrm{CO} 2$ to biomass. Berkely, University of California: 215.

Benvenuti, G., R. Bosma, M. Cuaresma, M. Janssen, M. J. Barbosa and R. H. Wijffels (2015). Selecting microalgae with high lipid productivity and photosynthetic activity under nitrogen starvation. Journal of applied phycology 27(4): 1425-1431.

Benvenuti, G., R. Bosma, A. J. Klok, F. Ji, P. P. Lamers, M. J. Barbosa and R. H. Wijffels (2015). Microalgal triacylglycerides production in outdoor batch-operated tubular PBRs. Biotechnol Biofuels 8.

Blanchard, G., J. Guarini, P. Richard, G. Ph and F. Mornet (1996). Quantifying the short-term temperature effect on light-saturated photosynthesis of intertidal microphytobenthos. Marine Ecology Progress Series 134: 309-313.

Borowitzka, M. A. (2005). Culturing microalgae in outdoor ponds. Algal culturing techniques, Academic Press: 205-218.

Bosma, R., E. v. Zessen, J. H. Reith, J. Tramper and R. H. Wijffels (2007). Prediction of volumetric productivity of an outdoor photobioreactor. Biotechnology and bioengineering 97(5): 1108-1120.

Bosma, R., K. Miazek, S. M. Willemsen, M. H. Vermue and R. H. Wijffels (2008). Growth inhibition of Monodus subterraneus by free fatty acids. Biotechnology and bioengineering 101(5): 1108-1114.

BP. (2009). "BP and Martek Biosciences Enter a Joint Development Agreement to Deliver Advanced Biodiesels" Retrieved 09.05.10.

Brentner, L. B., M. J. Eckelman and J. B. Zimmerman (2011). Combinatorial life cycle assessment to inform process design of industrial production of algal biodiesel. 45(16): 7060-7067. 
Brenton, P., G. Edwards-Jones and M. F. Jensen (2010). Case Study: Carbon Footprints of Tropical Food Products Calculated According to PAS 2050. Carbon Footprints and Food Systems. P. Brenton, G. Edwards-Jones and M. F. Jensen, The World Bank: 30-43.

Breuer, G., P. P. Lamers, D. E. Martens, R. B. Draaisma and R. H. Wijffels (2012). The impact of nitrogen starvation on the dynamics of triacylglycerol accumulation in nine microalgae strains. Bioresource Technology 124: 217-226.

Breuer, G., W. A. Evers, J. H. de Vree, D. M. Kleinegris, D. E. Martens, R. H. Wijffels and P. P. Lamers (2013) "Analysis of fatty acid content and composition in microalgae." Journal of visualized experiments: JoVE (), e50628 DOI: doi:10.3791/50628 (2013).

Breuer, G., P. P. Lamers, M. Janssen, R. H. Wijffels and D. E. J. B. t. Martens (2015). Opportunities to improve the areal oil productivity of microalgae. 186: 294-302.

Brown, T. M., P. G. Duan and P. E. Savage (2010). Hydrothermal Liquefaction and Gasification of Nannochloropsis sp. Energy \& Fuels 24: 3639-3646.

Buffet, L. (2017). How to make the Renewable Energy Directive (RED II) work for renewable electricity in transport. Transport \& Environment, Brussels.

Burgess, G. and J. G. Fernandez-Velasco (2008). Materials, operational energy inputs, and net energy ratio for photobiological hydrogen production. International Journal of Hydrogen energy 32(9): 1225-1234.

Burlew, J. S. (1953). Algal culture. From Laboratory to Pilot Plant, Carnegie Inst. Washington Publ 600.

Cabanelas, I. T. D., M. Zwart, D. M. M. Kleinegris, R. H. Wijffels and M. J. Barbosa (2016). Sorting cells of the microalga Chlorococcum littorale with increased triacylglycerol productivity. Biotechnol Biofuels 9.

Camacho, R., F, F. Acíen, FG, P. Sánchez, F, C. García, F and E. Molina Grima (1999). Prediction of Dissolved Oxygen and Carbon Dioxide Concentration Profiles in Tubular Photobioreactors for Microalgal Culture. Biotechnology and Bioengineering 62(1): 71-86.

Carlozzi, P. and G. Torzillo (1996). Productivity of Spirulina in a strongly curved tubular photobioreactor. Applied Microbiology and Biotechnology 45: 18-23.

Carlozzi, P. (2000). Hydrodynamic aspects and Arthrospira growth in two outdoor tubular undulating row reactors. Applied Microbiology and Biotechnology 54: 14-22.

Carvalho, A. P., L. A. Meireles and X. Malcata (2006). Microalgal Reactors: A Review of Enclosed System Designs and Performances. Biotechnology Progress 22(6): 1490-1506. 
Cavonius, L. R., N.-G. Carlsson and I. Undeland (2014). Quantification of total fatty acids in microalgae: comparison of extraction and transesterification methods. Analytical and Bioanalytical Chemistry 406(28): 7313-7322.

Chauton, M. S., K. I. Reitan, N. H. Norsker, R. Tveterås and H. T. Kleivdal (2015). A technoeconomic analysis of industrial production of marine microalgae as a source of EPA and DHA-rich raw material for aquafeed: Research challenges and possibilities. Aquaculture 436: 95-103.

Chini Zittelli, G., L. Rodolfi, N. Biondi and M. R. Tredici (2006). Productivity and photosynthetic efficiency of outdoor cultures of Tetraselmis suecica in annular columns. Aquaculture 261(3): 932-943.

Chini Zittelli, G., N. Biondi, L. Rodolfi and M. Tredici (2013). Photobioreactors for Mass Production of Microalgae.

Choi, K. J., Z. Nakhost, E. Bárzana and M. Karel (1987). Lipid content and fatty acid composition of green algae Scenedesmus obliquus grown in a constant cell density apparatus.

Christenson, L. and R. Sims (2011). Production and harvesting of microalgae for wastewater treatment, biofuels, and bioproducts. Biotechnology advances 29(6): 686-702.

Collares-Pereira, M. and A. Rabl (1979). The average distribution of solar radiationcorrelations between diffuse and hemispherical and between daily and hourly insolation values. Solar Energy 22(2): 155-164.

Coulson, J. M. and J. F. Richardson (1999). Piping and instrumentation. Chemical Enginering. J. M. Coulson, B. Richardson, J. R. Backhurst and J. H. Harker, Butterworth and Heinemann. 6: 358-364.

Cuaresma, M., M. Janssen, C. Vílchez and R. Wijffels, H. (2009). Productivity of Chlorella sorokiniana in a short light-path (SLP) panel photobioreactor under high irradiance. Biotechnology and bioengineering 104(2): 352-359.

Cuaresma, M., M. Janssen, E. J. van den End, C. Vilchez and R. H. Wijffels (2011). Luminostat operation: A tool to maximize microalgae photosynthetic efficiency in photobioreactors during the daily light cycle? Bioresource Technology 102(17): 7871-7878.

DACE (2002). Prijzenboekje. Leidschendam, Dutch Association of Cost Engineers.

de Mooij, T., G. de Vries, C. Latsos, R. H. Wijffels and M. J. A. r. Janssen (2016). Impact of light color on photobioreactor productivity. Algal research 15: 32-42. 
Del Campo, J. A., H. Rodríguez, J. Moreno, M. A. Vargas, J. Rivas and M. G. Guerrero (2001). Lutein production by Muriellopsis sp. in an outdoor tubular photobioreactor. Journal of Biotechnology 85(3): 289-295.

Doucha, J. and K. Lílvansky (2005). Productivity, CO2/O2 exchange and hydraulics in outdoor open high density microalgal (Chlorella sp.) photobioreactors operated in a Middle and Southern European climate. J Appl Phycol 18: 811-826.

Droop, M. R. (1974). The nutrient status of algal cells in continuous culture. Journal of the Marine Biological Association of the United Kingdom 54(04): 825-855.

Duboc, P., I. Marison and U. Von Stockar (1999). Quantitative calorimetry and biochemical engineering. Handbook of Thermal Analysis and Calorimetry. R. B. Kemp, Elsevier Science. From Macromolecules to Man.

Duffie, J. A. and W. A. Beckman (1991). Solar Engineering of Thermal Processes, John Wiley \& Sons.

Enzing, C., M. Ploeg, M. Barbosa and L. Sijtsma (2014). Microalgae-based products for the food and feed sector: an outlook for Europe. JRC Scientific and policy reports: 19-37.

EPA, U. (2002). Wastewater Technology Fact Sheet-Facultative Lagoons, EPA 832-F-022014.

Fawley, M. W., I. Jameson and K. P. Fawley (2015). The phylogeny of the genus Nannochloropsis (Monodopsidaceae, Eustigmatophyceae), with descriptions of N. australis sp. nov. and Microchloropsis gen. nov. Phycologia 54(5): 545-552.

Gates, B. (2020) "How we'll move around in a clean, green future." Sustainable Development Impact Summit.

Geider, R., H. Maclntyre and T. Kana (1997). Dynamic model of phytoplankton growth and acclimation: responses of the balanced growth rate and the chlorophyll a: carbon ratio to light, nutrient-limitation and temperature. Marine Ecology Progress Series 148: 187-200.

Gilbert, J. J., S. Ray and D. Das (2011). Hydrogen production using Rhodobacter sphaeroides (O.U. 001) in a flat panel rocking photobioreactor. International Journal of Hydrogen Energy 36(5): 3434-3441.

Goldman, J. C. (1979). Outdoor algal mass cultures - I. Applications. Water Research 13(1): 1-19.

Goldman, J. C. (1979). Outdoor algal mass cultures--II. Photosynthetic yield limitations. Water Research 13(2): 119-136. 
Grima, E. M., F. G. Camacho, J. A. S. Perez, J. U. Cardona, F. G. A. Fernandez and J. M. F. Sevilla (1994). Outdoor chemostat culture of Phaeodactylum tricornutum UTEX 640 in a tubular photobioreactor for the production of eicosapentaenoic acid. Biotechnology and Applied Biochemistry 20: 279-290.

Grima, E. M., E.-H. Belarbi, F. G. A. Fernandez, A. R. Medina and Y. Chisti (2003). Recovery of microalgal biomass and metabolites: process options and economics. Biotechnology Advances 20: 491-515.

Guccione, A., N. Biondi, G. Sampietro, L. Rodolfi, N. Bassi and M. R. Tredici (2014). Chlorella for protein and biofuels: from strain selection to outdoor cultivation in a Green Wall Panel photobioreactor. Biotechnology for Biofuels 7(1): 84 .

Guerra, L. T., O. Levitan, M. J. Frada, J. S. Sun, P. G. Falkowski and G. C. Dismukes (2013). Regulatory branch points affecting protein and lipid biosynthesis in the diatom Phaeodactylum tricornutum. Biomass and Bioenergy 59: 306-315.

Halfhide, T., A. Åkerstrøm, O. I. Lekang, H. R. Gislerød and S. J. Ergas (2014). Production of algal biomass, chlorophyll, starch and lipids using aquaculture wastewater under axenic and non-axenic conditions. Algal research 6: 152-159.

Hall, D. O., G. Acien Fernandez, E. Canizares Guerrero, K. K. Rao and E. Molina-Grima (2003). Outdoor Helical Tubular Photobioreactors for Microalgal Production: Modeling of Fluid dynamics and Mass Transfer and Assessment of Biomass Productivity Biotechnology and Bioengineering 82(1): 62-73.

Hase, R., H. Oikawa, C. Sasao, M. Morita and Y. Watanabe (2000). Photosynthetic production of microalgal biomass in a raceway system under greenhouse conditions in Sendai city. Journal Of Bioscience And Bioengineering 89(2): 157-163.

Hawkins, D. M. (2004). The problem of overfitting. Journal of chemical information and computer sciences 44(1): 1-12.

Ho, T.-Y., A. Quigg, Z. V. Finkel, A. J. Milligan, K. Wyman, P. G. Falkowski and F. M. M. Morel (2003). The elemental composition of some marine phytoplankton. J.Phycol. 39: 11451159.

Hsueh, H. T., W. J. Li, H. H. Chen and H. Chu (2009). Carbon bio-fixation by photosynthesis of Thermosynechococcus sp. CL-1 and Nannochloropsis oculta. Journal of Photochemistry and Photobiology B: Biology 95(1): 33-39.

Hu, Q., N. Kurano, M. Kawachi, I. Iwasaki and S. Miyachi (1998). Ultrahigh-cell-density culture of a marine green alga Chlorococcum littorale in a flat-plate photobioreactor. Applied Microbiology and Biotechnology 49(6): 655-662. 
Huesemann, M., B. Crowe, P. Waller, A. Chavis, S. Hobbs, S. Edmundson and M. Wigmosta (2016). A validated model to predict microalgae growth in outdoor pond cultures subjected to fluctuating light intensities and water temperatures. Algal Research 13: 195-206.

Hulatt, C. J. and D. N. Thomas (2011). Energy efficiency of an outdoor microalgal photobioreactor sited at mid-temperate latitude. Bioresource Technology 102(12): 66876695.

Hulatt, C. J., R. H. Wijffels, S. Bolla and V. Kiron (2017). Production of Fatty Acids and Protein by Nannochloropsis in Flat-Plate Photobioreactors. PloS one 12(1): e0170440.

Humphreys, K. K. (1991). Jelen's cost and optimization engineering. Singapore, McGrawHill.

Industrial_Assessment_Center (Year?) "Compressed air systems." 1-31.

Isabella, J. (2020) "A Short History of Aquaculture Innovation." Hakai Magazine.

Iwamoto, H. (2004). Industrial production of microalgal cell-mass and secondary productsmajor industrial species. Handbook of microalgal culture: biotechnology and applied phycology. A. Richmond, Blackwell Science: 255-263.

Janssen, J. (2018). Lipid metabolism in Nannochloropsis gaditana during nitrogen starvation. Ph.D., Wageningen University.

Janssen, M., J. Tramper, L. R. Mur and R. H. Wijffels (2003). Enclosed outdoor photobioreactors: Light regime, photosynthetic efficiency, scale-up, and future prospects. Biotechnology and bioengineering 81(2): 193-210.

Jiménez, C., B. R. Cossío and F. X. Niell (2003). Relationship between physicochemical variables and productivity in open ponds for the production of Spirulina: a predictive model of algal yield. Aquaculture 221(1-4): 331-345.

Jinkerson, R. E., R. Radakovits and M. C. J. B. Posewitz (2013). Genomic insights from the oleaginous model alga Nannochloropsis gaditana. 4(1): 37-43.

Johnson, D. B., L. C. Schideman, T. Canam and R. J. Hudson (2018). Pilot-scale demonstration of efficient ammonia removal from a high-strength municipal wastewater treatment sidestream by algal-bacterial biofilms affixed to rotating contactors. Algal Research 34: 143-153.

Jorquera, O., A. Kiperstok, E. A. Sales, M. Embiruçu and M. L. Ghirardi (2010). Comparative energy life-cycle analyses of microalgal biomass production in open ponds and photobioreactors. Bioresource Technology 101(4): 1406-1413. 
Klok, A. J., D. E. Martens, R. H. Wijffels and P. P. Lamers (2013). Simultaneous growth and neutral lipid accumulation in microalgae. Bioresource Technology 134: 233-243.

Klok, A. J., D. E. Martens, R. H. Wijffels and P. P. Lamers (2013). Simultaneous growth and neutral lipid accumulation in microalgae. Bioresour Technol 134.

Krauss, R. W. (1962). Mass culture of algae for food and other organic compounds. American Journal of Botany 49(4): 425-435.

Krishnamoorthy, S., P. Manickam and V. Muthukaruppan (2019). Evaluation of distillery wastewater treatability in a customized photobioreactor using blue-green microalgaeLaboratory and outdoor study. Journal of environmental management 234: 412-423.

Lane, J. (2018). Back to the Future All Over Again: ExxonMobil targets algae fuels at scale by 2025, as oil prices rise. Biofuels digest. 2018.

Laurens, L. and J. D. J. I. B. McMillan (2017). State of Technology Review-Algae Bioenergy.

Laurens, L. M. L., M. Quinn, S. Van Wychen, D. W. Templeton, E. J. J. A. Wolfrum and B. Chemistry (2012). Accurate and reliable quantification of total microalgal fuel potential as fatty acid methyl esters by in situ transesterification. 403(1): 167-178.

Laws, E. A., K. L. Terry, J. Wickman and M. S. f. Chalup (1983). A simple algal production system designed to utilize the flashing light effect. Biotechnology and Bioengineering 25 : 2319-2325.

Lee, Y.-K. (2001). Microalgal mass culture systems and methods: Their limitation and potential Journal of Applied Phycology 13: 307-315.

Lehr, F. and C. Posten (2009). Closed photo-bioreactors as tools for biofuel production. Current Opinion in Biotechnology 20(3): 280-285.

Marquez, F. J., K. Sasaki, N. Nishio and S. Nagai (1995). Inhibitory effect of oxygen accumulation on the growth of Spirulina platensis. Biotechnology Letters 17(2): 225-228.

Mascarelli, A. L. (2009). Gold rush for algae. Nature 461(7263): 460-461.

Masojídek, J. and O. Prasil (2010). The development of microalgal biotechnology in the Czech Republic.

Masy, J.-C., M. Cournil and M. Lance (1995). Agglomeration of alumina powders: A turbidimetric study. Chemical Engineering \& Technology 18(6): 425-433.

MDNA (2008). Climatological report 2008, Meteorological Service of the Netherlands Antilles and Aruba: 37. 
Meiser, A., U. Schmid-Staiger and W. Trosch (2004). Optimization of eicosapentaenoic acid production by Phaeodactylum tricornutum in the flat panel airlift (FPA) reactor. Journal of Applied Phycology 16: 215-225.

Michels, M. H., M. Vaskoska, M. H. Vermuë and R. H. Wijffels (2014). Growth of Tetraselmis suecica in a tubular photobioreactor on wastewater from a fish farm. Water research 65 : 290-296.

Michels, M. H. A., A. J. van der Goot, N. H. Norsker and R. H. Wijffels (2010). Effects of shear stress on the microalgae Chaetoceros muelleri. Bioprocess and Biosystems Engineering 33(8): 921-927.

Moheimani, N. R. and M. Borowitzka, A. (2007). Limits to productivity of the alga Pleurochrysis carterae (Haptophyta) grown in outdoor raceway ponds. Biotechnology and bioengineering 96(1): 27-36.

Molina Grima, E., J. A. S. Perez, F. G. Camacho, J. L. G. Sanchez, F. G. A. Fernandez and D. L. Alonso (1994). Outdoor culture of Isochrysis galbana ALII-4 in a closed tubular photobioreactor. Journal of Biotechnology 37: 159-166.

Molina Grima, E., J. A. Sanchez Pérez, F. Garcia Camacho, J. M. Fernández Sevilla and F. G. Acién Fernandez (1996). Productivity analysis of outdoor chemostat culture in tubular airlift photobioreactors. Journal of Applied Phycology 8: 369-380.

Molina Grima, E., F. García Camacho, J. A. Sánchez Pérez, F. G. Acíen Fernandez and J. M. Fernández Sevilla (1997). Evaluation of photosynthetic effiency in microalgal cultures using averaged irradiance. Enzyme and Microbial Technology 21: 375-381.

Moreno, J., M. n. Vargas, H. Rodrìguez, J. Rivas and M. G. Guerrero (2003). Outdoor cultivation of a nitrogen-fixing marine cyanobacterium, Anabaena sp. ATCC 33047. Biomolecular Engineering 20(4-6): 191-197.

Morweiser, M., O. Kruse, B. Hankamer and C. Posten (2010). Developments and perspectives of photobioreactors for biofuel production. Applied Microbiology and Biotechnology 87(4): 1291-1301.

Mulbry, W., S. Kondrad, C. Pizarro and E. Kebede-Westhead (2008). Treatment of dairy manure effluent using freshwater algae: algal productivity and recovery of manure nutrients using pilot-scale algal turf scrubbers. Bioresource technology 99(17): 8137-8142.

Mulders, K. J., J. H. Janssen, D. E. Martens, R. H. Wijffels and P. P. J. A. R. Lamers (2014). Effect of biomass concentration on secondary carotenoids and triacylglycerol (TAG) accumulation in nitrogen-depleted Chlorella zofingiensis. 6: 8-16.

Müller, P., X.-P. Li and K. K. Niyogi (2001). Non-photochemical quenching. A response to excess light energy. Plant physiology 125(4): 1558-1566. 
Münkel, R., U. Schmid-Staiger, A. Werner, T. J. B. Hirth and bioengineering (2013).

Optimization of outdoor cultivation in flat panel airlift reactors for lipid production by Chlorella vulgaris. 110(11): 2882-2893.

Nash, C. E. (2011). Farming the Sea (1880-1920). The History of Aquaculture, Blackwell Publishing: $69-78$.

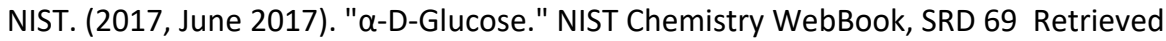
12/10/2018, 2018.

NOAA. (2020). "Trends in Atmospheric Carbon Dioxide." Retrieved 04-12-2020, 2020, from https://www.esrl.noaa.gov/gmd/ccgg/trends/mlo.html.

Norsker, N.-H., M. J. Barbosa, M. H. Vermuë and R. H. Wijffels (2011). Microalgal production - A close look at the economics. Biotechnology Advances 29: 24-27.

Norsker, N.-H., M. J. Barbosa, M. H. Vermuë and R. H. Wijffels (2012). On Energy Balance and Production Costs in Tubular and Flat Panel Photobioreactors.

Technikfolgenabschätzung - Theorie und Praxis 21(1): 8.

Norsker, N. H., M. Michiels, P. M. Slegers, G. L. A. M. Swinkels, M. J. Barbosa and R. H. Wijffels (2019). Productivity of Nannochloropsis oceanica in an industrial closely spaced flat panel photobioreactor. Algal Research 43: 101632.

Norwegian_Seafood_Council (2020) "Norwegian seafood exports top NOK 107 billion in 2019."

Okutsu, A. (2018). Jet biofuel mass production to begin in Japan. Nikkei Asian Review.

Olaizola, M. and C. Grewe (2019). Commercial microalgal cultivation systems. Grand Challenges in Algae Biotechnology, Springer: 3-34.

Oltermann, P. (2020). The wurst is over: why Germany now loves to go vegetarian. The Guardian, Guardian news and media Ltd.

Oswald, W. J. (2003). My sixty years in applied algology. Journal of Applied Phycology 15: 99-106.

Pegallapati, A. and N. Nirmalakhandan (2011). Energetic evaluation of an internally illuminated photobioreactor for algal cultivation. Biotechnology Letters 33(11): 2161-2167.

Pelletier, G. (2002). Solrad_ver12.xls. Olympia, WA, Washington State Dept. of Ecology: A solar position and radiation calculator for Microsoft Excel/VBA.

Photonsystems. (2018). "FMT 150." Retrieved 05/12/2018, 2018. 
Pimentel, D. and T. W. Patzek (2005). Ethanol Production Using Corn, Switchgrass, and Wood; Biodiesel Production Using Soybean and Sunflower. Natural Resources Research 14(1): 65-75.

Posten, C. (2009). Design principles of photo-bioreactors for cultivation of microalgae. Eng Life Sci 9.

Posten, C. and C. Walter (2012). Microalgal Biotechnology: Potential and Production.

Prentice, I. C., G. Farquhar, M. Fasham, M. Goulden, M. Heimann, V. Jaramillo, H. Kheshgi, C. LeQuéré, R. Scholes and D. W. Wallace (2001). The carbon cycle and atmospheric carbon dioxide. Climate Change: The scientific basis. . J. T. Houghton, Ding, Y., Griggs, D.J., Noguer, M., van der Linden, P.J., Dai, X., Maskell, K. and Johnson, C.A. Cambridge UK, Cambridge University Press: 185-237.

Pruvost, J., G. Van Vooren, G. Cogne and J. Legrand (2009). Investigation of biomass and lipids production with Neochloris oleoabundans in photobioreactor. Bioresource Technology 100(23): 5988-5995.

Pruvost, J., V. Goetz, A. Artu, P. Das and H. Al Jabri (2019). Thermal modeling and optimization of microalgal biomass production in the harsh desert conditions of State of Qatar. Algal research 38: 101381.

Pulz, O., N. Gerbsch and R. Buchholz (1995). Light energy supply in plate-type and light diffusing optical fiber bioreactors. Journal of Applied Phycology 7: 145-149.

Pulz, O. and K. Scheibenbogen (1998). Photobioreactors: Design and performance with respect to light energy input. T. Scheper. Berlin Heidelberg, Springer-Verlag: 123-152.

Pushparaj, B., E. Pelosi, M. R. Tredici, E. Pinzani and R. Materassi (1997). An integrated culture system for outdoor production of microalgae and cyanobacteria. Journal of Applied Phycology 9: 113-119.

Qiang, H., H. Guterman and A. Richmond (1996). Physiological characteristics of Spirulina platensis (cyanobacteria) cultured at ultrahigh cell densities. Journal of Phycology 32(6): 1066-1073.

Qiang, H. and A. Richmond (1996). Productivity and photosynthetic efficiency of Spirulina platensis as affected by light intensity, algal density and rate of mixing in a flat plate photobioreactor. Journal of Applied Phycology 8(2): 139-145.

Qiang, H., D. Faiman and A. Richmond (1998). Optimal tilt angles of enclosed reactors for growing photoautotrophic microorganisms outdoors. Journal of Fermentation and Bioengineering 85(2): 230-236. 
Qiang, H., D. Faiman, A. J. J. o. f. Richmond and bioengineering (1998). Optimal tilt angles of enclosed reactors for growing photoautotrophic microorganisms outdoors. 85(2): 230-236.

Qiang, H., Y. Zarmi and A. s. Richmond (1998). Combined effects of light intensity, lightpath and culture density on output rate of Spirulina platensis (Cyanobacteria). European Journal of Phycology 33(2): 165-171.

Qiang, H., S. Milton, J. Eric, G. Maria, P. Matthew, S. Michael and D. Al (2008). Microalgal triacylglycerols as feedstocks for biofuel production: perspectives and advances. The Plant Journal 54(4): 621-639.

Quinn, J. C., T. Yates, N. Douglas, K. Weyer, J. Butler, T. H. Bradley and P. J. Lammers (2012). Nannochloropsis production metrics in a scalable outdoor photobioreactor for commercial applications. Bioresource Technology 117: 164-171.

Raso, S., B. Van Genugten, M. Vermuë and R. H. Wijffels (2012). Effect of oxygen concentration on the growth of Nannochloropsis sp. at low light intensity. Journal of applied phycology 24(4): 863-871.

Remmers, I. M., A. Hidalgo-Ulloa, B. Brandt, W. A. Evers, R. H. Wijffels and P. P. J. B. t. Lamers (2017). Continuous versus batch production of lipids in the microalgae Acutodesmus obliquus. 244: 1384-1392.

Remmers, I. M., R. H. Wijffels, M. J. Barbosa and P. P. J. T. i. b. Lamers (2018). Can We Approach Theoretical Lipid Yields in Microalgae? 36(3): 265-276.

Richmond, A. and J. U. Grobbelaar (1986). Factors affecting the output rate of Spirulina platensis with reference to mass cultivation. Biomass 10(4): 253-264.

Richmond, A., E. Lichtenberg, B. Stahl and A. Vonshak (1990). Quantitative assessment of the major limitations on productivity of Spirulina platensis in open raceways. Journal of Applied Phycology 2(3): 195-206.

Richmond, A. (2000). Microalgal biotechnology at the turn of the millennium: A personal view Journal of Applied Phycology 12: 441-451.

Riet, K. v. t. and J. Tramper (1991). Basic Bioreactor Design. New York, Marcel Dekker, Inc.

Rodolfi, L., G. C. Zittelli, N. Bassi, G. Padovani, N. Biondi, G. Bonini and M. R. Tredici (2009). Microalgae for oil: Strain selection, induction of lipid synthesis and outdoor mass cultivation in a low-cost photobioreactor. Biotechnology and Bioengineering 102(1): 100112.

Ruiz, J., G. Olivieri, J. de Vree, R. Bosma, P. Willems, J. H. Reith, M. H. M. Eppink, D. M. M. Kleinegris, R. H. Wijffels and M. J. Barbosa (2016). Towards industrial products from microalgae. Energy \& Environmental Science 9(10): 3036-3043. 
Ruiz-Domínguez, M. C., I. Vaquero, V. Obregón, B. de la Morena, C. Vílchez and J. M. J. J. o. a. p. Vega (2015). Lipid accumulation and antioxidant activity in the eukaryotic acidophilic microalga Coccomyxa sp.(strain onubensis) under nutrient starvation. 27(3): 1099-1108.

Rumin, J., E. Nicolau, R. G. d. O. Junior, C. Fuentes-Grünewald, K. J. Flynn and L. Picot (2020). A bibliometric analysis of microalgae research in the world, Europe, and the European Atlantic area. Marine drugs 18(2): 79.

Satel-light (2008). Satel-light, the European Database of Daylight and Solar radiation, The Satel-light project. A European Union funded database.

Schenk, P. M., S. R. Thomas-Hall, E. Stephens, U. C. Marx, J. H. Mussgnug, C. Posten, O. Kruse and B. J. B. r. Hankamer (2008). Second generation biofuels: high-efficiency microalgae for biodiesel production. 1(1): 20-43.

Sciandra, A., J. Gostan, Y. Collos, C. Descolas-Gros, C. Leboulanger, V. Martin-Jezequel, M. Denis, D. Lefevre, C. Copin-Montegut and B. Avril (1997). Growth-Compensating Phenomena in Continuous Cultures of Dunaliella tertiolecta Limited Simultaneously by Light and Nitrate. Limnology and Oceanography 42(6): 1325-1339.

Scragg, A. H., A. M. Illman, A. Carden and S. W. Shales (2002). Growth of microalgae with increased calorific values in a tubular bioreactor. Biomass and Bioenergy 23(1): 67-73.

Sergejevová, M., J. R. Malapascua, J. Kopecký and J. Masojídek (2015). Photobioreactors with internal illumination. Algal Biorefineries, Springer: 213-236.

Sforza, E., A. Bertucco, T. Morosinotto and G. M. Giacometti (2012). Photobioreactors for microalgal growth and oil production with Nannochloropsis salina: From lab-scale experiments to large-scale design. Chemical Engineering Research and Design 90(9): 11511158.

Sheehan, J., T. Dunahay, J. Benemann and P. Roessler (1998). A Look Back at the U.S. Department of Energy's Aquatic Species Program-Biodiesel from Algae, .

Shoener, B., I. Bradley, R. Cusick and J. Guest (2014). Energy positive domestic wastewater treatment: the roles of anaerobic and phototrophic technologies. Environmental Science: Processes \& Impacts 16(6): 1204-1222.

Sierra, E., F. G. Acién, J. M. Fernández, J. L. García, C. González and E. Molina (2008). Characterization of a flat plate photobioreactor for the production of microalgae. Chemical Engineering Journal 138(1-3): 136-147.

Singh, A. and S. I. Olsen (2011). A critical review of biochemical conversion, sustainability and life cycle assessment of algal biofuels. Applied Energy 88(10): 3548-3555. 
Slade, R., A. J. B. Bauen and bioenergy (2013). Micro-algae cultivation for biofuels: cost, energy balance, environmental impacts and future prospects. 53: 29-38.

Slegers, P. M., R. H. Wijffels, G. van Straten and A. J. B. van Boxtel (2011). Design scenarios for flat panel photobioreactors. Applied Energy 88(10): 3342-3353.

Slegers, P. M., G. Olivieri, E. Breitmayer, L. Sijtsma, M. Eppink, R. H. Wijffels and J. H. Reith (2020). Design Of Value Chains For Microalgal Biorefinery At Industrial Scale: Process Integration And Techno-Economic Analysis. Frontiers in Bioengineering and Biotechnology 8: 1048.

Solovchenko, A., I. Khozin-Goldberg, L. Recht and S. Boussiba (2010). Stress-Induced Changes in Optical Properties, Pigment and Fatty Acid Content of Nannochloropsis sp.: Implications for Non-destructive Assay of Total Fatty Acids. Marine Biotechnology: 1-9.

Solovchenko, A., A. Lukyanov, O. Solovchenko, S. Didi-Cohen, S. Boussiba and I. KhozinGoldberg (2014). Interactive effects of salinity, high light, and nitrogen starvation on fatty acid and carotenoid profiles in Nannochloropsis oceanica CCALA 804. European Journal of Lipid Science and Technology 116(5): 635-644.

Sonneveld, P. J. and G. L. A. M. Swinkels (2005). Micro-V covering materials for photovoltaic cells 20th European photovoltaic solar energy conference and exhibition, 6-10 June 2005. . Barcelona, Spain.

Sonneveld, P. J., G. Swinkels, G. P. A. Bot and G. Flamand (2010). Feasibility study for combining cooling and high grade energy production in a solar greenhouse. Biosystems Engineering 105(1): 51-58.

Sousa, C., L. de Winter, M. Janssen, M. H. Vermuê and R. H. Wijffels (2012). Growth of the microalgae Neochloris oleoabundans at high partial oxygen pressures and sub-saturating light intensity. Bioresource Technology 104: 565-570.

Spoehr, H. and H. Milner (1947). Chlorella as a source of food. Carnegie Inst. Wash. Ybk 47: 100-103.

Spolaore, P., C. Joannis-Cassan, E. Duran and A. Isambert (2006). REVIEW: Commercial Applications of Microalgae. Journal of Bioscience and Bioengineering 101(2): 9.

Spolaore, P., C. Joannis-Cassan, E. Duran and A. Isambert (2006). Optimization of Nannochloropsis oculata growth using the response surface method. Journal of Chemical Technology \& Biotechnology 81(6): 1049-1056.

Stephens, E., I. L. Ross, Z. King, J. H. Mussgnug, O. Kruse, C. Posten, M. A. Borowitzka and B. Hankamer (2010). An economic and technical evaluation of microalgal biofuels. Nat Biotech 28(2): 126-128. 
Sukenik, A. (1991). Ecophysiological considerations in the optimization of eicosapentaenoic acid production by Nannochloropsis sp. (Eustigmatophyceae). Bioresource Technology 35(3): 263-269.

Swinkels, G. L. A. M., P. J. Sonneveld and G. P. A. Bot (2001). Improvement of Greenhouse Insulation with Restricted Transmission Loss through Zigzag Covering Material. Journal of Agricultural Engineering Research 79(1): 91-97.

Terry, K. L., J. Hirata and E. A. Laws (1985). Light-limited, nitrogen-limited, and phosphoruslimited growth of phaeodactylum-tricornutum bohlin strain tfx-1 - chemical-composition, carbon partitioning, and the diel periodicity of physiological processes. Journal of Experimental Marine Biology and Ecology 86(1): 85-100.

TNO. (2020). "Organization." Retrieved 2020, from https://www.tno.nl/en/abouttno/organisation/.

Torzillo, G., B. Pushparaj, F. Bocci, W. Balloni, R. Materassi and G. Florenzano (1986). Production of Spirulina biomass in closed photobioreactors. Biomass 11(1): 61-74.

Torzillo, G., P. Carlozzi, B. Pushparaj, E. Montaini and R. Materassi (1993). A two-plane tubular photobioreactor for outdoor culture of Spirulina. Biotechnology and Bioengineering 42(7): 891-898.

Tredici, M., G. C. Zittelli and L. Rodolfi (2010). Photobioreactors. Encyclopedia of Industrial Biotechnology M. Flickinger, Wiley. 7.

Tredici, M., N. Bassi, M. Prussi, N. Biondi, L. Rodolfi, G. C. Zittelli and G. J. A. e. Sampietro (2015). Energy balance of algal biomass production in a 1-ha "Green Wall Panel" plant: how to produce algal biomass in a closed reactor achieving a high net energy ratio. 154: 11031111.

Tredici, M., R., G. Chini Zittelli, S. Biagiolini and R. Materassi (1993). Novel photobioreactors for the mass cultivation of Spirulina spp. Monaco, MONACO, Musée ocanographique.

Tredici, M. R. and G. C. Zittelli (1998). Efficiency of sunlight utilization: Tubular versus flat photobioreactors. Biotechnology and bioengineering 57(2): 187-197.

Ugwu, C. U., J. C. Ogbonna and H. Tanaka (2005). Light/dark cyclic movement of algal culture (Synechocystis aquatilis) in outdoor inclined tubular photobioreactor equipped with static mixers for efficient production of biomass. Biotechnol. Lett. 27(2): 3.

Ugwu, C. U., J. C. Ogbonna and H. Tanaka (2005). Light/dark cyclic movement of algal culture (Synechocystis aquatilis) in outdoor inclined tubular photobioreactor equipped with static mixers for efficient production of biomass. Biotechnology Letters 27: 75-78. 
Ugwu, C. U., H. Aoyagi and H. Uchiyama (2008). Photobioreactors for mass cultivation of algae. Bioresource Technology 99(10): 4021-4028.

USEPA. (2010). "Meteorological Data: California." Retrieved March, 04.th, 2010, from http://www.epa.gov/ceampubl/tools/metdata/ca/index.html.

Van Wagenen, J., T. W. Miller, S. Hobbs, P. Hook, B. Crowe and M. Huesemann (2012). Effects of light and temperature on fatty acid production in Nannochloropsis salina. Energies 5(3): 731-740.

Vejrazka, C., M. Janssen, M. Streefland and R. H. Wijffels (2011). Photosynthetic efficiency of Chlamydomonas reinhardtii in flashing light. Biotechnology and bioengineering 108(12): 2905-2913.

Vieira, V. V. (2017). Value and size of Algae Biomass sector in Europe in 2016 and relevance of standardization. CEN BT WG 218 workshop, Brussels.

Vonshak, A., A. Abeliovich, S. Boussiba, S. M. Arad and A. Richmond (1982). Production of Spirulina biomass: Effects of environmental factors and population density. Biomass 2: 175185.

Vonshak, A. and A. Richmond (1988). Mass production of the blue-green alga Spirulina: An overview. Biomass 15(4): 233-247.

Vree, J. H., R. Bosma, M. Janssen, M. J. Barbosa and R. H. Wijffels (2015). Comparison of four outdoor pilot-scale photobioreactors. Biotechnology for Biofuels 8(1): 1-12.

Waltz, E. (2009). Biotech's green gold? Nature America 27(1): 15-18.

Wan, C., F.-W. Bai and X.-Q. Zhao (2013). Effects of nitrogen concentration and media replacement on cell growth and lipid production of oleaginous marine microalga Nannochloropsis oceanica DUT01. Biochemical Engineering Journal 78: 32-38.

Wassink, E. (1970). Plant Physiology and some aspects of future agriculture. Netherlands Journal of Agricultural Science 18(1): 49-61.

Wassink, E. C., B. Kok and J. L. P. v. Oorschot (1953). The efficiency of light-energy conversion in chlorella cultures as compared with higher plants. Algal culture from laboratory to pilot plant. J. S. Burlew. Washington, d. c, Carnegie Institution of Washington publication \# 600

:55-62.

Weissman, J. C., R. P. Goebel and J. R. Benemann (1988). Photobioreactor design: Mixing, carbon utilization, and oxygen accumulation. Biotechnology and Bioengineering 31: 336344. 
Weyer, K. M., D. R. Bush, A. Darzins and B. D. Willson (2010). Theoretical Maximum Algal Oil Production. BioEnergy Research 3(2): 204-213.

Wijffels, R. H., M. J. Barbosa and M. H. M. Eppink (2010). Microalgae for the production of bulk chemicals and biofuels. Biofuels, Bioproducts and Biorefining 4(3): 287-295.

Wikipedia (2020). World oil market chronology from 2003, Wikipeia, The Free Encyclopedia.

Wollmann, F., S. Dietze, J. U. Ackermann, T. Bley, T. Walther, J. Steingroewer and F. Krujatz (2019). Microalgae wastewater treatment: Biological and technological approaches. Engineering in Life Sciences 19(12): 860-871.

Work, V. H., S. D'Adamo, R. Radakovits, R. E. Jinkerson and M. C. Posewitz (2012). Improving photosynthesis and metabolic networks for the competitive production of phototroph-derived biofuels. Current Opinion in Biotechnology 23(3): 290-297.

Xu, L., P. J. Weathers, X. R. Xiong and C. Z. Liu (2009). Microalgal bioreactors: Challenges and opportunities. Engineering in Life Sciences 9(3): 178-189.

Zhang, K., N. Kurano and S. Miyachi (1999). Outdoor culture of a cyanobacterium with a vertical flat-plate photobioreactor: effects on productivity of the reactor orientation, distance setting between the plates, and culture temperature. Applied Microbiology and Biotechnology 52(6): 781-786.

Zhang, K., S. Miyachi and N. Kurano (2001). Photosynthetic performance of a cyanobacterium in a vertical flat-plate photobioreactor for outdoor microalgal production and fixation of CO2. Biotechnology Letters 23(1): 21-26.

Zhang, K., S. Miyachi and N. Kurano (2001). Evaluation of a vertical flat-panel photobioreactor for outdoor biomass production and carbon dioxide bio-fixation:effects of reactor dimensions, irradiation and cell concentration on the biomass productivity and irradiation utilization efficiency. Applied Microbiology and Biotechnology 55: 428-433.

Zhang, K., N. Kurano and S. Miyachi (2002). Optimized aeration by carbon dioxide gas for microalgal production and mass transfer characterization in a vertical flat-plate photobioreactor. Bioprocess and Biosystems Engineering 25(2): 97-101.

Zou, N. and A. Richmond (1999). Effect of light-path lengh in outdoor flat plate reactors on output rate of cell mass and on EPA in Nannochloropsis sp. Journal of Biotechnology 70: 351-356. 


\section{Author curriculum vitae}

Full name

Date of birth

Citizenship

Address

Phone number

e-mail

Education

1983

\section{Employment history}

1983

1985

1989

1991

1995, 6 months

1997

2006

2008

2012 - present
Niels-Henrik Norsker.

August 27, 1954.

Danish

Nyvej 33B, 5762 Vester Skerninge, Denmark

$+4530915786$

nhnorsker@biotopic.dk

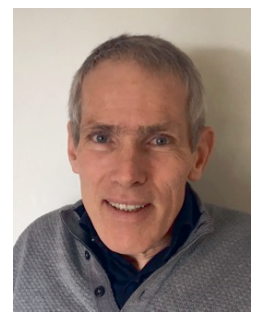

Copenhagen University: Environmental biology

Institute of Plant Physiology. M.Sc. thesis: Nitrate uptake and continuous

cultivation of Monochrysis lutheri

Danish Technical University: Experimental fermentation technology.

Gladsaxe Gymnasium: Teaching proficiency.

Frederiksberg Technical school, Høje Tåstrup higher preparatory course, Danish Teachers Academy: Biology, chemistry, plant physiology.

Denconsult A/S, consulting engineers. Project manager.

Ålborg University. Research assistant, sewer biofilm kinetics.

BioProcess Aps. Microalgal photobioreactor production. General manager.

Copenhagen Urban Ecology Center Copenhagen Municipality. Manager.

BioProcess A/S (Scotland, Iceland). Research manager.

Køge Gymnasium, Frederiksborg Gymnasium, VUC syd: high school lecturer (biology and chemistry).

Wageningen University, Bioprocess engineering. Microalgal researcher.

BioTopic, Norsker Investigaciones S.L. . General manager. (Microalgal research

and development projects in the Netherlands, Belgium and Spain). 


\section{Acknowledgements:}

The study described in Chapter 2 and 3 was carried out with support from the Dutch power company, DELTA n.v. and from the program "Towards Biosolar Cells", the Netherlands.

The study described in Chapter 4 was carried out with support from Proviron, Belgium and the Belgian Ministry of the Environment.

The study described in Chapters 5 and 6 was carried out with support from Neste Corporation, Finland.

Mass spectroscopy in Chapter 5 was carried out by Twente University, the Netherlands.

Cover photograph by Jeroen de Vree.

Printed by Proefschriftmaken, Prinsenlaan 2, 3732 GN De Bilt, the Netherlands. 

